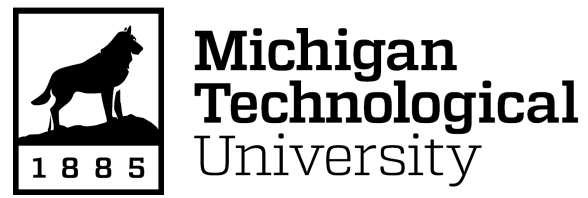

Michigan Technological University Digital Commons @ Michigan Tech

Dissertations, Master's Theses and Master's Reports

2018

INTERPLAY OF IONIC TRANSPORT AND CRYSTAL FACETS IN LITHIUM-ION BATTERY CATHODES

Wentao Yao

Michigan Technological University, wentaoya@mtu.edu

Copyright 2018 Wentao Yao

Recommended Citation

Yao, Wentao, "INTERPLAY OF IONIC TRANSPORT AND CRYSTAL FACETS IN LITHIUM-ION BATTERY

CATHODES", Open Access Dissertation, Michigan Technological University, 2018.

https://doi.org/10.37099/mtu.dc.etdr/698

Follow this and additional works at: https://digitalcommons.mtu.edu/etdr 


\title{
INTERPLAY OF IONIC TRANSPORT AND CRYSTAL FACETS IN LITHIUM-ION BATTERY CATHODES
}

\author{
By
}

Wentao Yao

\begin{abstract}
A DISSERTATION
Submitted in partial fulfillment of the requirements for the degree of DOCTOR OF PHILOSOPHY

In Mechanical Engineering-Engineering Mechanics
\end{abstract}

MICHIGAN TECHNOLOGICAL UNIVERSITY

2018

(C) 2018 Wentao Yao 
This dissertation has been approved in partial fulfillment of the requirements for the Degree of DOCTOR OF PHILOSOPHY in Mechanical Engineering-Engineering Mechanics.

Department of Mechanical Engineering-Engineering Mechanics

Dissertation Advisor: Reza Shahbazian-Yassar

Committee Member: $\quad$ Craig Friedrich

Committee Member: $\quad$ Gregory Odegard

Committee Member: $\quad$ Stephen Hackney

Department Chair: William Predebon 


\section{Table of Contents}

Preface

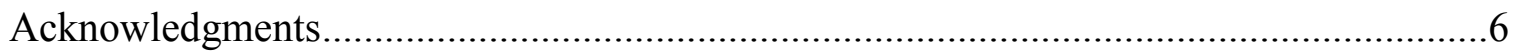

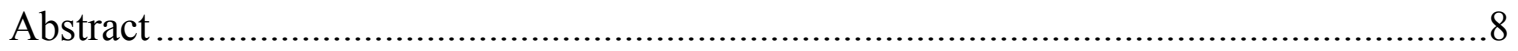

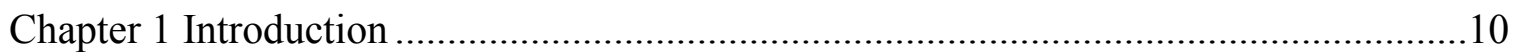

$1.1 \mathrm{LiCoO}_{2}$ structure and lithium ion diffusion pathways ......................................10

$1.2 \beta-\mathrm{MnO}_{2}$ crystal structure and application in energy storage devices..................13

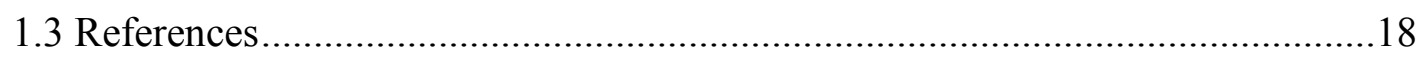

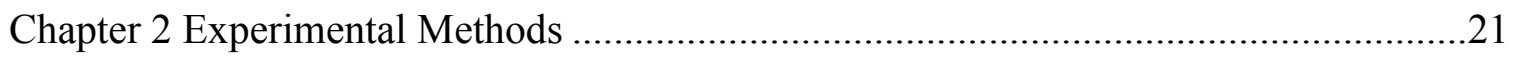

2.1 Conductive atomic force microscopy (C-AFM) ………………….................21

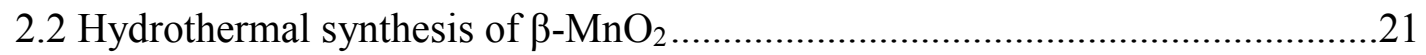

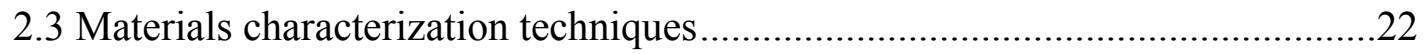

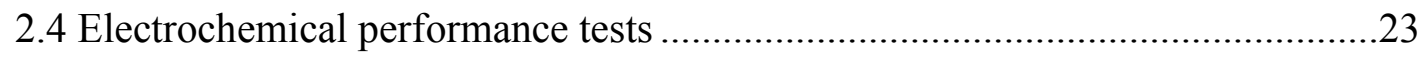

Chapter 3 Localized mechanical stress induced ionic redistribution in a layered $\mathrm{LiCoO}_{2}$

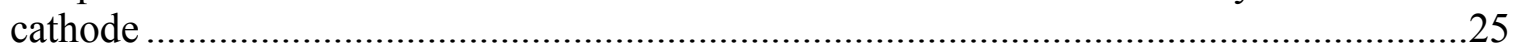

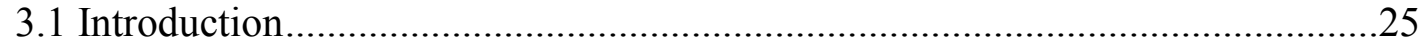

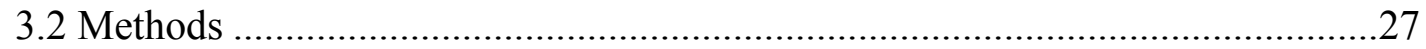

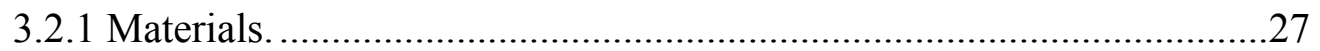

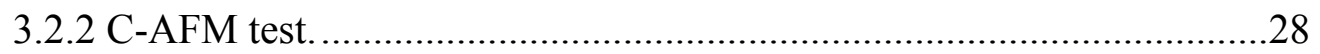

3.2.3 Calibration of deflection sensitivity and spring constant .....................28

3.2.4 Dynamic force ramping..................................................................29

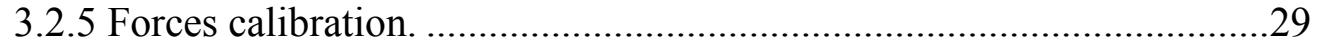

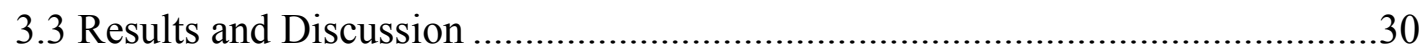

3.3.1 C-AFM tests on the layered $\mathrm{LiCoO}_{2}$ cathode.......................................30

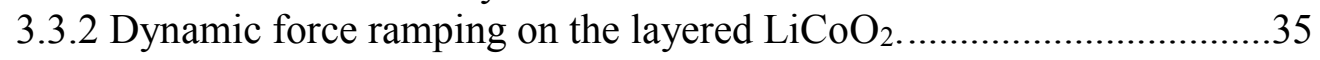

3.3.3 Comparison C-AFM test on the HOPG................................................38

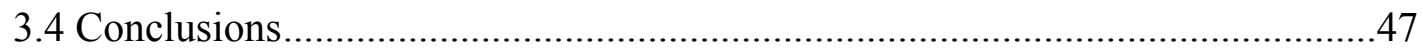

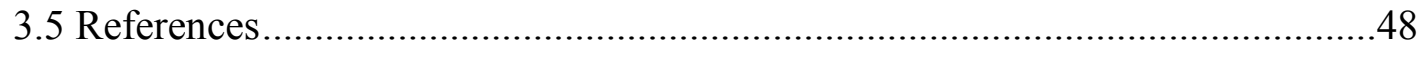

Chapter 4 Energy-driven surface evolution in beta- $\mathrm{MnO}_{2}$ structures...............................52

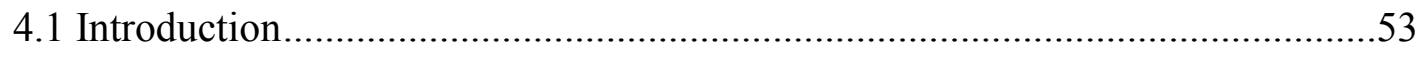

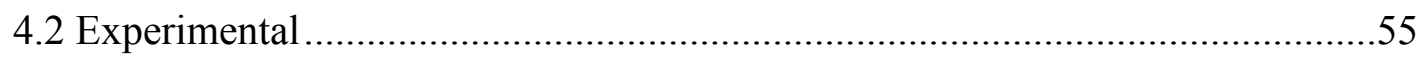

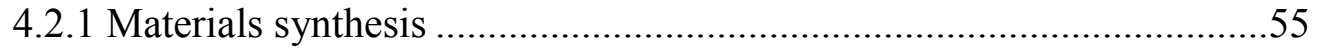




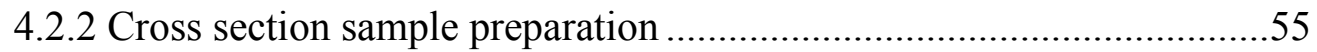

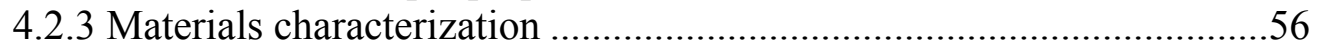

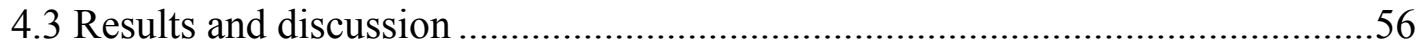

4.3.1 Facet evolution of the $\beta-\mathrm{MnO}_{2}$ nanowires...........................................56

4.3.2 Facet evolution of the $\beta-\mathrm{MnO}_{2}$ microrods.............................................61

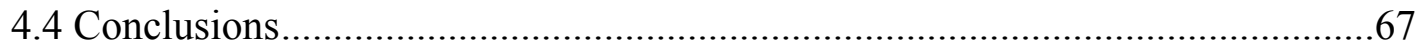

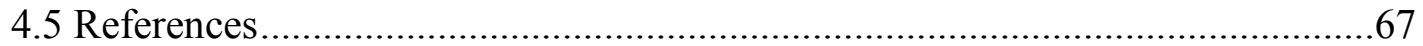

Chapter 5 Cations controlled growth of $\beta-\mathrm{MnO}_{2}$ crystals with tunable facets for

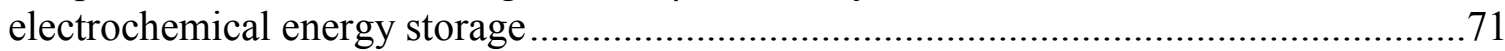

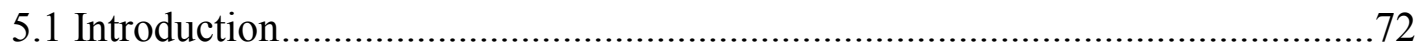

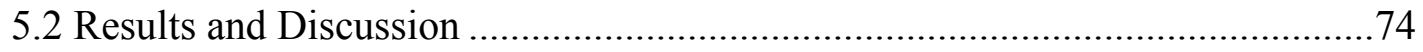

5.2.1 Growth mechanism of $\beta-\mathrm{MnO}_{2}$ crystals .............................................74

$5.2 .2 \mathrm{~K}^{+}$cations effect on the morphology of $\beta-\mathrm{MnO}_{2}$ structure ...................79

5.2.3 Crystal surfaces identification ...........................................................82

5.2.4 The role of $\mathrm{K}^{+}$cations during the morphology evolution of $\beta-\mathrm{MnO}_{2} \ldots 86$

5.2.5 Electrochemical performance as the cathode materials for $\mathrm{Li}$-ion batteries 90

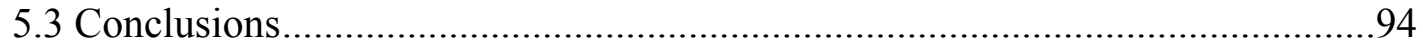

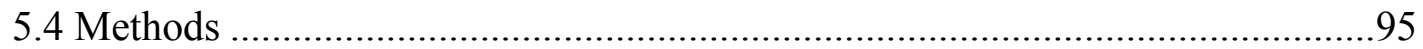

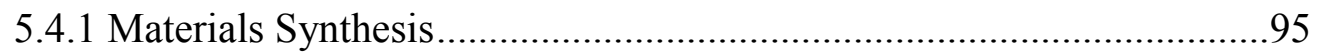

5.4.2 Cross-section sample preparation.........................................................95

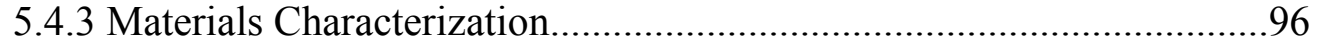

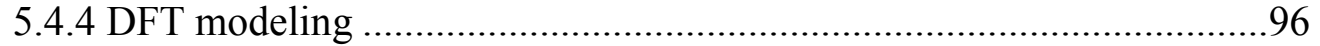

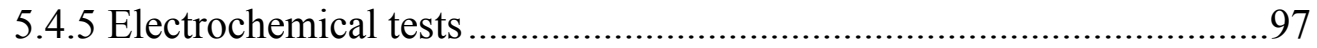

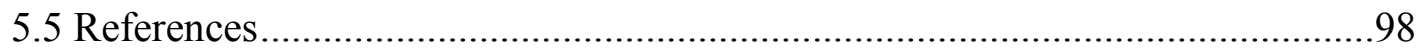

Chapter 6 Facet-dependent electrochemical and catalytic performance of beta $\mathrm{MnO}_{2}$

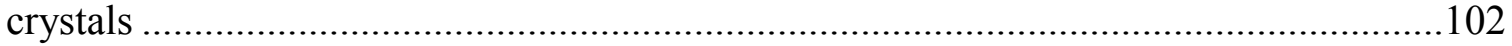

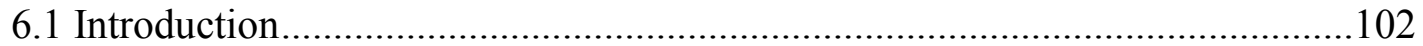

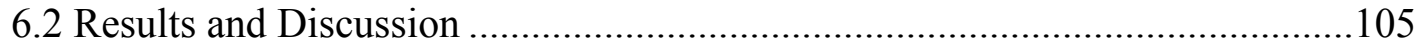

6.2.1 Materials synthesis and characterization .............................................105

6.2.2 Facets effect on the electrochemical performance of $\beta-\mathrm{MnO}_{2} \ldots \ldots \ldots \ldots . .110$

6.2.3 Facets effect on the catalytic performance of $\beta-\mathrm{MnO}_{2} \ldots \ldots \ldots \ldots \ldots \ldots \ldots \ldots . . . . .114$

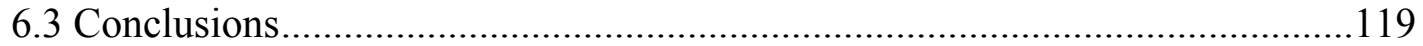

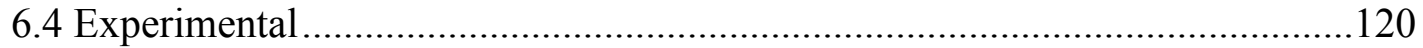

6.4.1 Materials Synthesis......................................................................120

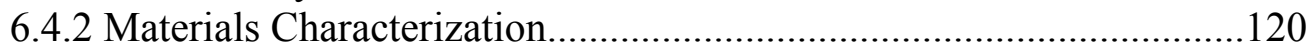

6.4.3 Cross section sample preparation ......................................................121

6.4.4 Electrochemical performance test ....................................................121 
Chapter 7 Conclusion...

Chapter 8 Future Work

130

8.1 Effect of $\mathrm{Mg}^{2+}$ cations on the formation of todorokite $\mathrm{MnO}_{2}$........................130

8.2 Whether $\mathrm{Na}^{+}$ions can intercalate into [1×1] tunnel of $\beta-\mathrm{MnO}_{2} \ldots \ldots \ldots \ldots \ldots \ldots . . . . . . .132$

8.3 Effect of crystal phases on the performance of $\mathrm{MnO}_{2}$ as the cathode for $\mathrm{Mg}$-ion batteries

8.4 References 136

Appendix A. Supporting information for Chapter 3 138

Appendix B. Supporting information for Chapter 4 145

Appendix C. Supporting information for Chapter 5 148

Appendix D. Supporting information for Chapter 7 154 


\section{Preface}

This preface serves as an introduction to the contents of the four main chapters in this dissertation. These chapters cover four specific projects which I was focusing on during my Ph.D. study at Michigan Tech from Fall 2013 to Spring 2018. The main topic of this dissertation is the study of mechanical forces and exposed crystal facets on the lithium ion diffusion in layered and tunnel-based cathode materials.

Chapter 3 includes the study of mechanical forces effects on the lithium-ion redistribution in a layered $\mathrm{LiCoO}_{2}$ cathode using conductive atomic force microscopy (C-AFM). $\mathrm{Mr}$. Wentao Yao designed and conducted the main experiments under the direction of Dr. Fei Long (Michigan Technological University) and Dr. Reza Shahbazian-Yassar (the University of Illinois at Chicago). The full content of this chapter is published in print in ACS Applied Materials \& Interfaces in 2016. (2016, 8(43), 29391-29399. DOI: 10.1021/acsami.6b07491).

Chapter 4 covers the study of crystal facet evolution in tunnel-based beta $\mathrm{MnO}_{2}$ cathode material. Beta $\mathrm{MnO}_{2}$ crystals with both nanowire and microrod morphologies were adopted to reveal the effect of hydrothermal reaction time on the lateral surface evolution in these two morphologies. A mutual surface evolution mechanism was revealed. Mr. Wentao Yao conducted the main experiments under the direction of Dr. Reza Shahbazian-Yassar. Dr. Yifei Yuan (Argonne National Laboratory) contributed to the characterization of the structures. The full content of this chapter has been published in print in Nano Research in 2018. (2018, 11(1), 206-215. DOI:10.1007/s12274-017-1620-5). 
Chapter 5 covers the study of cations effect on the morphology evolution in the beta $\mathrm{MnO}_{2}$ structure and its effect on lithium ion diffusion as the cathode material. The growth mechanism, as well as the cations effect, were thoroughly investigated combining experimental techniques and computational simulations. Mr. Wentao Yao conducted the main experimental under the direction of Dr. Reza Shahbazian-Yassar. Dr. Gregory Odegard (Michigan Tech) conducted the density functional theory simulations for this project. The full content of this chapter has been published in print in Nano Energy in 2018. (2018, 48, 301-311. DOI: 10.1016/j.nanoen.2018.03.057).

Chapter 6 summarized the study of crystal facets effect on the electrochemical and catalytic performance of beta $\mathrm{MnO}_{2}$ as the electrode for supercapacitor as well as the cathode catalyst for lithium-air batteries. Mr. Wentao Yao synthesized the materials and conducted the supercapacitor experiments. Dr. Yifei Yuan and Dr. Guoqiang Tan (Argonne National Laboratory) conducted the lithium air tests. The full content of this chapter is currently under preparation and will be submitted for publication in the future. 


\section{Acknowledgments}

I would like to take this opportunity to express my great appreciation to the help and directions I have received from many faculties, staffs, and colleagues during my Ph.D. study at Michigan Tech as well as the University of Illinois at Chicago. These projects cannot be completed and published without your kind support and help.

I would like to express my great thanks to my advisor, Dr. Reza Shahbazian-Yassar, who brought me to the Energy and Nano Science lab in 2013 and put tremendous efforts to get me well-trained as a professional researcher, which I couldn't imagine five years ago. I would not be able to reach this stage without his kind guidance and continuous support. During my Ph.D. study, I am deeply impressed by his open mind towards individual research interests, his excellent writing and presentation skills, as well as his outstanding social skills.

I would also like to express my great thanks to Dr. Craig Friedrich for his generous financial support for my tuitions and part of the research expenses at Michigan Tech during the past three years. My Ph.D. projects cannot be finished without these financial support opportunities. Many thanks to my committee member, Dr. Gregory Odegard, who have spent lots of time helping with computational simulations. Additional thanks to my committee members, Dr. Stephen Hackney, for his kind support to my Ph.D. studies.

Additional thanks to my colleagues and staffs at MTU and UIC for their support to my experiments. I would like to thank my colleagues Dr. Fei Long, Dr. Yifei Yuan, Dr. Hasti Asayesh, Mr. Soroosh Sharifi, Mr. Zhennan Huang, Mr. Meng Cheng, Mr. Boao Song, Mr. 
Ram Deivanayagam, and the staffs Mr. Owen mills, Dr. Edward Laitila, Dr. Alan Nicholls, Dr. Fengyuan Shi for the kind help with my experiments, technical training, and timely support.

Special thanks to my family, especially my wife, Dr. Zhimin Song, for her great support whenever there are difficulties or troubles in my research projects and personal life.

Wentao Yao

Michigan Technological University, Jun 2018 


\begin{abstract}
Cathode crystals in lithium-ion batteries act as the host for the (de)intercalation for lithium ions. The diffusion of lithium ions in layered or tunneled cathode crystals is highly selective along certain crystal plans or directions. Exposed facets of the cathode crystals can greatly affect the diffusion of lithium ions within the electrode, which in return affect the electrochemical performances of the batteries. In this dissertation, layered $\mathrm{LiCoO}_{2}$ and tunnel-based beta $\mathrm{MnO}_{2}$ were selected as two individual systems to evaluate the effect of mechanical stress and exposed crystal facets on the lithium ion diffusion in these two cathode materials, respectively.
\end{abstract}

For the layered $\mathrm{LiCoO}_{2}$ cathode, the effect of mechanical stress on lithium ion diffusion in layered $\mathrm{LiCoO}_{2}$ cathode was investigated using conductive atomic force microscopy (C-AFM). Higher localized mechanical stress could induce more active lithium ion redistribution along the grain boundaries than the grain interiors. The external stress field within $100 \mathrm{nN}$ could induce the resistive-switching effect of the $\mathrm{LiCoO}_{2}$ cathode.

For the tunnel-based beta $\mathrm{MnO}_{2}$ cathode, high-resolution transmission electron microscopy (TEM) and scanning transmission electron microscopy (STEM) were used to real the lateral facets evolution mechanism in both nanowire and microrod morphologies. The evolution of lateral facets was found to follow the shift from $\{100\}$ facets to $\{110\}$ facets because of the relatively high surface energies of the $\{100\}$ facets compared with $\{110\}$ facets. Further studies show the micro-sized beta $\mathrm{MnO}_{2}$ was formed through oriented attachment and subsequent direct phase transition from $\mathrm{a}-\mathrm{K}_{\mathrm{x}} \mathrm{MnO}_{2}$ nanowires. The 
concentration of potassium cations $\left(\mathrm{K}^{+}\right)$could be used to control the morphology of the obtained beta $\mathrm{MnO}_{2}$ crystals. The morphology changed from bipyramid prism to octahedron when the concentration of $\mathrm{K}^{+}$increase from $0.02 \mathrm{M}$ to $0.09 \mathrm{M}$. The role of $\mathrm{K}^{+}$ cations was revealed to affect both the formation and phase transition of a- $\mathrm{K}_{\mathrm{x}} \mathrm{MnO}_{2}$ intermediate. The two morphologies were identified with highly exposed $\{100\}$ and $\{111\}$ facets, respectively.

The effect of crystal facets on the electrochemical and catalytic performance of beta $\mathrm{MnO}_{2}$ was further studies based on the application of these two morphologies in lithiumion batteries, supercapacitors, and lithium-air batteries systems, respectively. The results show, the highly exposed $\{111\}$ facets offered beta $\mathrm{MnO}_{2}$ higher lithium ion mobility inside the structure and thus better rate performance because of highly exposed open tunnels. The $\{100\}$ facets of beta $\mathrm{MnO}_{2}$ offered higher specific capacitance as the electrode for supercapacitors, which is due to the highly exposed Mn centers on the $\{100\}$ facets compared with $\{111\}$. As the cathode catalyst for lithium-air batteries, both facets showed effective catalytic activities in reducing the charge and discharge overpotential; the $\{111\}$ facets of beta $\mathrm{MnO}_{2}$ was, for the first time, revealed to catalyze a solution-based mechanism for the formation of $\mathrm{LiO}_{2}$ intermediate even in a low donor number electrolyte. 


\section{Chapter 1 Introduction}

The past twenty years have seen the tremendous increase in the consuming of rechargeable lithium-ion batteries in portable electronic devices, thanks to its high energy density compared with traditional lead acid, Ni-Cd, and Ni-MH batteries. A standard rechargeable lithium-ion battery mainly composed of cathode, anode, and the separator soaked with an organic electrolyte. The charge-discharge of the battery is based on the reversible intercalation and deintercalation of lithium ions from the anode and cathode. The first generation of commercial cathode is layered $\mathrm{LiCoO}_{2}$, which was discovered by J.B. Goodenough in $1980 .{ }^{1}$ Followed that, several suitable cathode materials were developed, including lithium manganese oxide $\left(\mathrm{LiMn}_{2} \mathrm{O}_{4}\right)$ with a spinel structure, lithium-ion phosphate $\left(\mathrm{LiFePO}_{4}\right)$ with an olivine structure, and lithium nickel manganese cobalt oxide ( $\left.\mathrm{Li}(\mathrm{Ni}, \mathrm{Mn}, \mathrm{Co}) \mathrm{O}_{2}\right)$. Unlike layered $\mathrm{LiCoO}_{2}$, which provides two-dimensional pathways for lithium ion (de)intercalation, $\beta-\mathrm{MnO}_{2}$ crystal offers highly ordered one-dimensional tunnel for the accommodation of lithium ions.

\section{1 $\mathrm{LiCoO}_{2}$ structure and lithium ion diffusion pathways}

Lithium cobalt dioxide $\left(\mathrm{LiCoO}_{2}\right)$ has two crystal forms: one is a cubic spinel-relate structure with a space group $F d 3 m$; the other is a hexagonal layered structure with a space group $R 3 m$ and an $\mathrm{ABCABC}$ oxygen stacking (O3 phase, as shown in Figure 1). ${ }^{2}$ Layered $\mathrm{LiCoO}_{2}$ is generally used for the lithium-ion battery application. The layered $\mathrm{LiCoO}_{2}$ composed of one $\mathrm{CoO}_{2}$ layer and one lithium layer, which offer two-dimensional pathways for lithium (de)intercalation along the (001) plane. Structure disorders are commonly introduced to improve its electrochemical performance due to its improved stability 
towards lithium (de)intercalation with defective structures. ${ }^{2}$ Several polymorphs were explored for the layered structure based on the introduce of stacking faults defects. Different from the standard $\mathrm{O} 3$ phase with $\mathrm{ABCABC}$ stacking, $\mathrm{O} 2$-type $\mathrm{LiCoO}_{2}$ can be prepared by ion-exchange from P2-type $\mathrm{Na}_{0.7} \mathrm{CoO}_{2}$, which gives a different oxygen stacking of ABACABAC. Another polymorph is O4-type $\mathrm{LiCoO}_{2}$, which is composed of an intergrowth of both $\mathrm{O} 2$ - and $\mathrm{O} 3$-type $\mathrm{LiCoO}_{2}{ }^{3}$
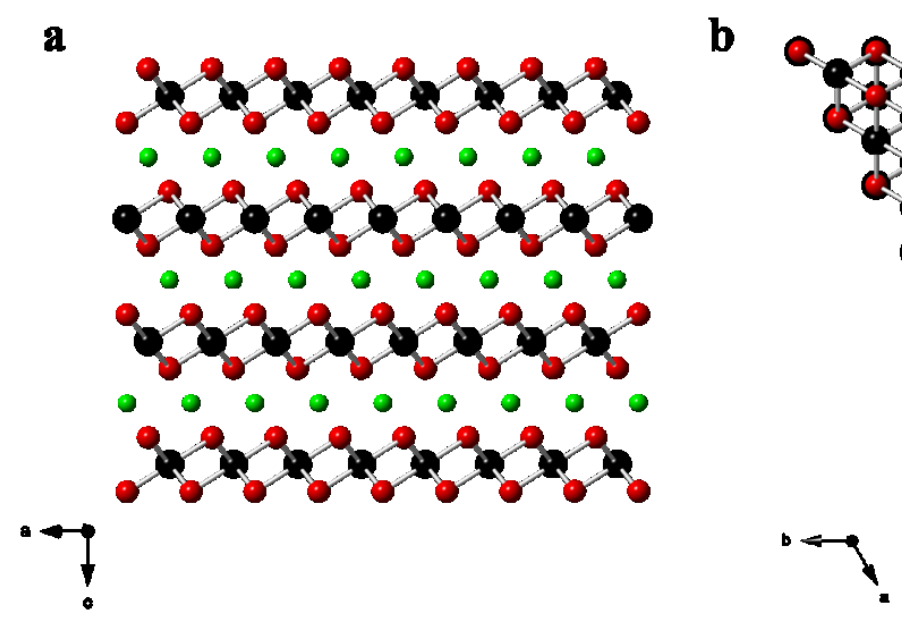

Figure 1-1 Schematic images showing the atom arrangement of layered O3-type $\mathrm{LiCoO}_{2}$ viewed along the (a) [010] (illustrating the alternate arrangement of $\mathrm{CoO}_{2}$ and lithium layers) and the (b) [001] directions (illustrating the hexagonal arrangement of $\mathrm{Co}$ and $\mathrm{O}$ atoms).

Because of the highly selective pathways along (001) plane for the diffusion of lithium ion inside layered $\mathrm{LiCoO}_{2}$, the size, morphology, and orientation of the crystal can greatly affect its electrochemical performances. ${ }^{4-7}$ Nano-sized $\mathrm{LiCoO}_{2}$ can generally maintain higher capacity under higher charge-discharge rate due to the increased surface areas and reduced diffusion pathways. ${ }^{5}$ Nanoscale understanding of the diffusion of lithium 
ions inside the layered $\mathrm{LiCoO}_{2}$ and how other factors (e.g. mechanical stress) affect the diffusion are of great importance for the development of this important cathode material.
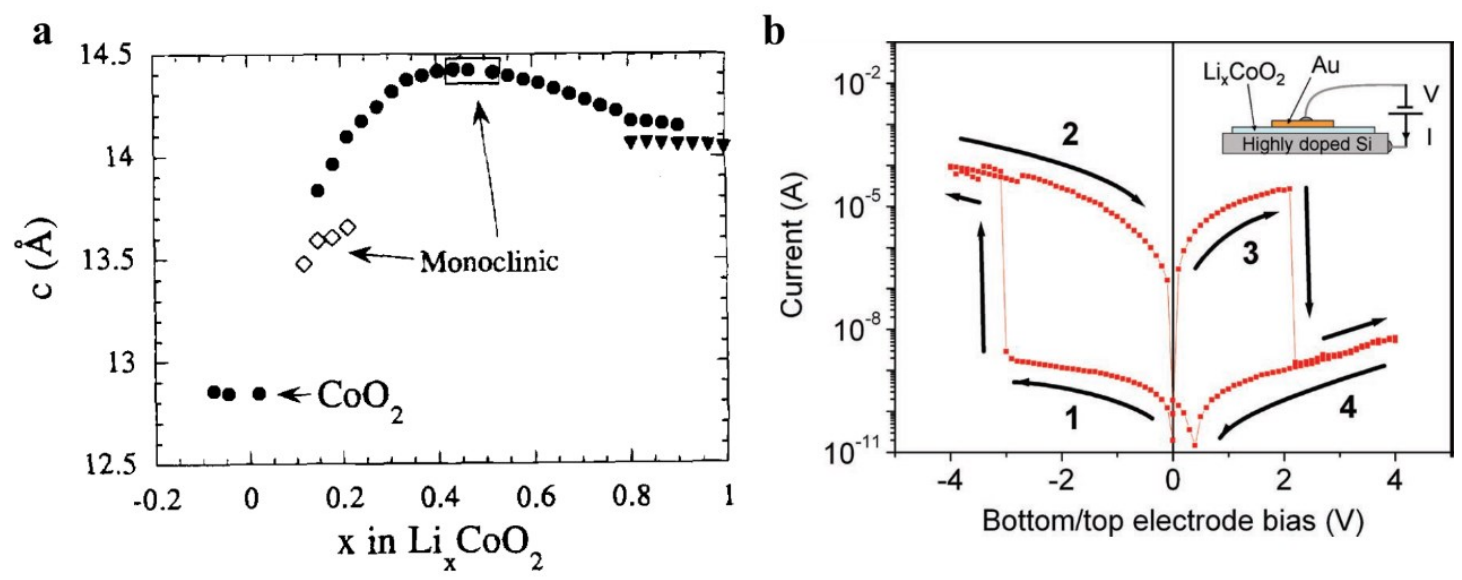

Figure 1-2 (a) Variation of $c$ lattice parameters detected by in situ XRD during delithiation of $\mathrm{LiCoO}_{2}$. Triangles and circles indicate hexagonal phases, while diamonds represent monoclinic phases. ${ }^{8}(\mathrm{~b})$ The I-V curves measured from a $\mathrm{Li}_{\mathrm{x}} \mathrm{CoO}_{2}$ thin film within a voltage range from $-4 \mathrm{~V}$ to $+4 \mathrm{~V}$. Resistive switching happens around $-3 \mathrm{~V}$ and $+2.5 \mathrm{~V} .{ }^{9}$

Upon initial delithiation of the layered $\mathrm{Li}_{\mathrm{x}} \mathrm{CoO}_{2}$, two major changes were observed, including a lattice expansion along the $c$-axis $(0.5<\mathrm{x}<1)$ and a conductivity change from semi-conductive to metallic $(0.75<\mathrm{x}<0.94) .{ }^{8,9}$ Both changes can be detected using atomic force microscopy (AFM). The lattice expansion can induce changes of the electrode surface morphology, which is detectable in the topography images acquired from AFM. The conductivity change can also be detected based on conductive AFM measurement. Based on conductive AFM, N. Balke et al first developed the electrochemical strain microscopy (ESM) technique, which allows direct nanoscale mapping of lithium ion diffusion in the layered $\mathrm{LiCoO}_{2}$ cathode. It was found that the concentrated electric field provided from the AFM tip can trigger localized redistribution (or (de)intercalation) of lithium ions. The 
localized lithium ion deficiency induces lattice expansions which can be further tracked by the AFM tip. On the other hand, the localized lithium ion deficiency can also be tracked based on the conductivity change by using conductive AFM tip. Zhu et al adopted C-AFM to study the lithium ion diffusion in layered $\mathrm{LiCoO}_{2}$ and found higher lithium ion mobility along the grain boundaries than the grain interiors. ${ }^{10}$

The volume expansion along delithiation of the $\mathrm{LiCoO}_{2}$ crystal will introduce mechanical stresses to the adjacent crystals inside the electrode. This is generally inevitable especially for the electrode materials with large volume expansion upon lithium (de)intercalation. The generated stress field could further affect the (de)lithiation of lithium ions. To study the effect of mechanical stress on lithium ion diffusion in the layered $\mathrm{LiCoO}_{2}$, the localized mechanical force was applied using the AFM cantilever beam. The applied forces are generally within hundreds of nano-newtons. The force applied can be calibrated on standard substrates. Because C-AFM is running in contact mode, with the AFM tip directly touching the surface of the sample, localized forces, therefore, can be controlled and applied along with the electric field.

\section{2 $\mathrm{\beta}-\mathrm{MnO}_{2}$ crystal structure and application in energy storage devices}

Manganese dioxide $\left(\mathrm{MnO}_{2}\right)$ has wide application in various energy storage devices, including supercapacitors, metal-air batteries, rechargeable lithium/sodium ion batteries, and fuel cells. The different crystal structures of $\mathrm{MnO}_{2}$ can be differentiated based on their different connection of the $\left[\mathrm{MnO}_{6}\right]$ octahedra units, which forms either layered or tunneled structures. Unlike layered $\mathrm{LiCoO}_{2}$, beta $\mathrm{MnO}_{2}$ with rutile structure shows one-dimensional 
tunnels $(2.3 \AA \times 2.3 \AA)$ which can accommodate lithium ions (de)intercalation (Figure 2). The diffusion pathways for lithium ions inside beta $\mathrm{MnO}_{2}$ is also highly selective along the tunnel direction. The morphology or crystal facets of beta $\mathrm{MnO}_{2}$ would greatly affect its electrochemical performance as a lithium ion battery cathode.

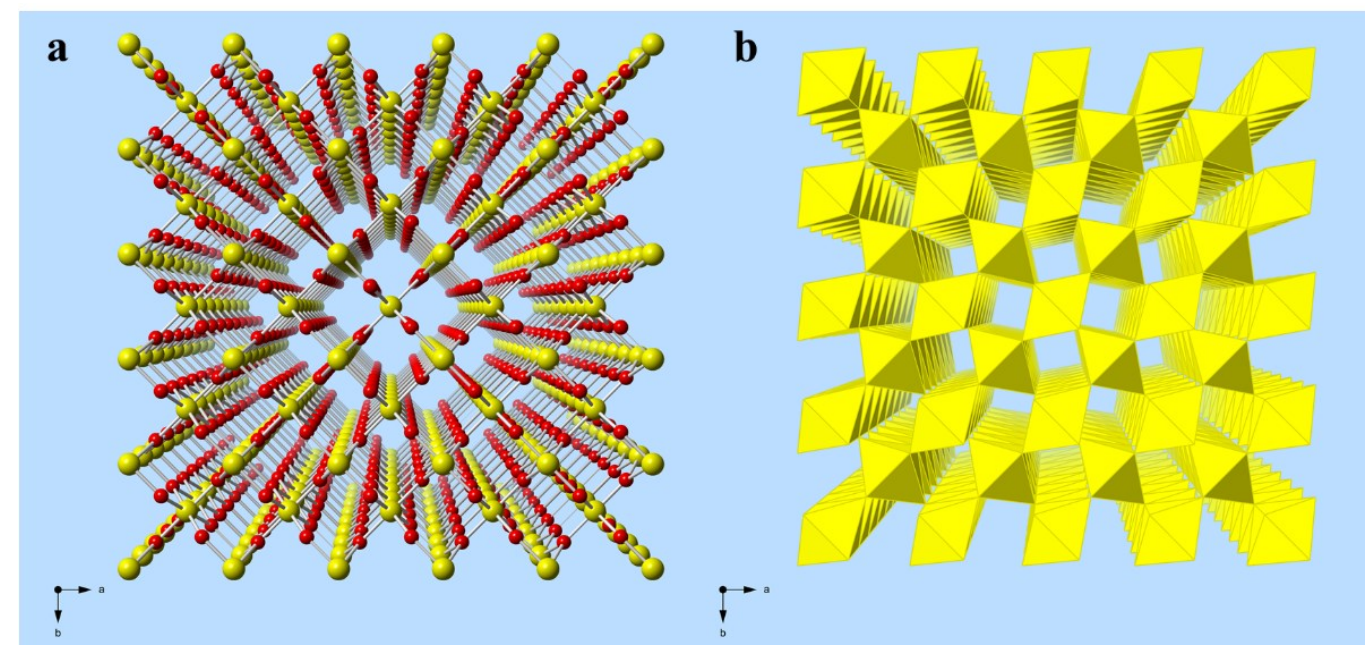

Figure 1-3 Schematic atom model illustrating the tunnel structure of beta $\mathrm{MnO}_{2}$ with (a) ball-stick and (b) polyhedral units.

The bulk level $\beta-\mathrm{MnO}_{2}$ is generally inactive for lithium ion (de)intercalation due to the poor kinetics for lithium ion diffusion. The intercalation of lithium ions was reported to be less than $\mathrm{Li} / \mathrm{Mn}=0.3$ at room temperature. ${ }^{11,12}$ However, when fabricated into nanostructures, the intercalation of lithium ion into beta $\mathrm{MnO}_{2}$ was reported to increase to $\mathrm{Li} / \mathrm{Mn}=1.15$ with a high capacity of $320 \mathrm{mAh} \cdot \mathrm{g}^{-1}$ (cut-off voltage $\left.=1.0 \mathrm{~V}\right) .{ }^{11}$ The standard charge-discharge curve of the $\beta-\mathrm{MnO}_{2} /$ acetylene black composite was shown in Figure 3a. Three discharge plateaus near $4 \mathrm{~V}, 2.8 \mathrm{~V}$ and $1.6 \mathrm{~V}$ were observed. The $2.8 \mathrm{~V}$ plateau was related to the intercalation of lithium ions in the $[1 \times 1]$ tunnels; while the $4 \mathrm{~V}$ and $1.6 \mathrm{~V}$ were related to a structural phase transition from rutile $\beta-\mathrm{MnO}_{2}$ to spinel $\mathrm{LiMn}_{2} \mathrm{O}_{4}$; and this 
structure change did not induce a great decrease in charge-discharge capacity in nanostructured $\beta-\mathrm{MnO}_{2}{ }^{11}$
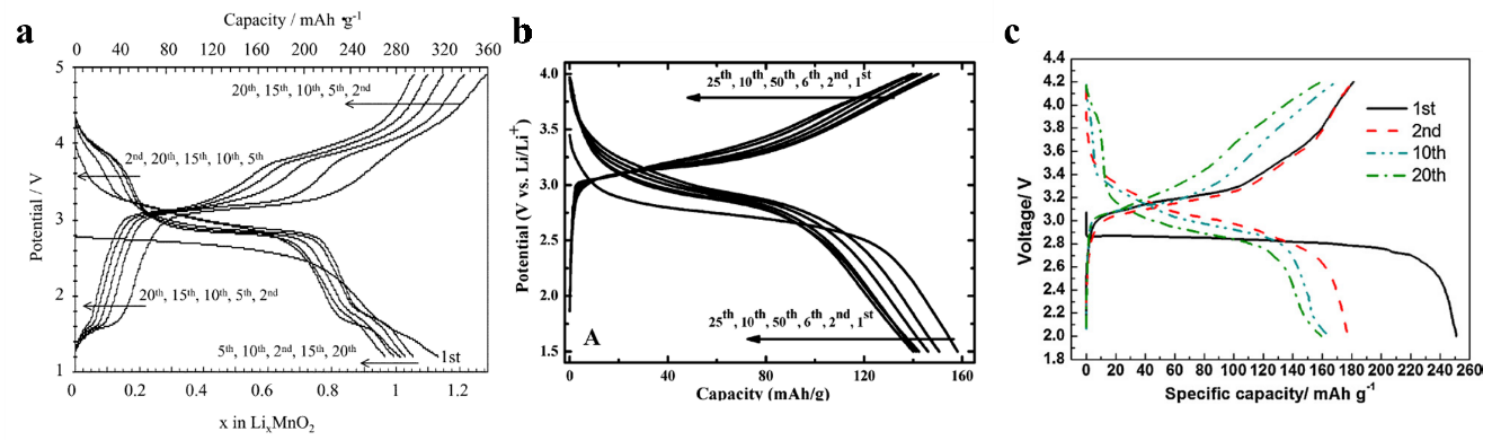

Figure 1-4 (a) Charge-discharge curve of the $\beta-\mathrm{MnO}_{2} /$ Acetylene black composite at 0.1 $\mathrm{mA} / \mathrm{cm}^{2}{ }^{11}$ (b) Charge-discharge curve of mesoporous $\beta-\mathrm{MnO}_{2}$ at a current rate of 0.4 $\mathrm{mA} / \mathrm{cm}^{2}{ }^{13}$ (c) Charge-discharge curve of hollow macroporous $\beta-\mathrm{MnO}_{2}$ at a current density of $10 \mathrm{~mA} \cdot \mathrm{g}^{-1} \cdot 14$

Recently, mesoporous and hollow structured $\beta-\mathrm{MnO}_{2}$ were also reported to show considerable high capacity towards lithium ion (de)intercalation. With a mesoporous structure, Luo et al obtained a high capacity of $219.4 \mathrm{mAh} \cdot \mathrm{g}^{-1}$ at a current density of 0.03 $\mathrm{mA} / \mathrm{cm}^{2}$ (cut-off voltage $=1.5 \mathrm{~V}$ ), owing to the larger specific surface area for the intercalation of lithium ion and the reduced lithium ion diffusion paths (Figure 4b). ${ }^{13}$ And more importantly, only the (de)intercalation plateaus showed up in the charge-discharge curve. By adjusting the pore size and increasing the surface area of the mesoporous structure, Jiao et al obtained a higher capacity around $284 \mathrm{mAh} \cdot \mathrm{g}^{-1}$ (equivalent to $\mathrm{Li}_{0.92} \mathrm{MnO}_{2}$ ) at a current density of $15 \mathrm{~mA} \cdot \mathrm{g}^{-1}$ (cut-off voltage $=2.0 \mathrm{~V}$ ) and the phase transition to $\mathrm{LiMn}_{2} \mathrm{O}_{4}$ spinel was avoided. ${ }^{14}$ The reduced phase transition from rutile to spinel may be related to the special structure of mesoporous $\beta-\mathrm{MnO}_{2}$. The large pore sizes 
provide enough spaces to accommodate the volume expansion upon lithium intercalation in the $[1 \times 1]$ tunnels.

Hollow bipyramid $\beta-\mathrm{MnO}_{2}$ also reported high capacities as the cathode for lithiumion batteries. ${ }^{14-16}$ Huang et al obtained $251 \mathrm{mAh} \cdot \mathrm{g}^{-1}$ initial capacity at $10 \mathrm{~mA} \cdot \mathrm{g}^{-1}$ (cut-off voltage $=2.0 \mathrm{~V})$. An irreversible phase transition to orthorhombic $\mathrm{Li}_{\mathrm{x}} \mathrm{MnO}_{2}$ was observed during the initial lithium intercalation. ${ }^{14}$ This irreversible phase transition to the orthorhombic structure was also reported by Zhan et al. ${ }^{16}$ The high capacity of this morphology was mainly due to the hollow structure which opens more sites for the (de)intercalation of lithium ions and improved the contact area of the electrode with the electrolyte. Irreversible phase transitions appear to be inevitable for both nanostructured and the hollow bipyramid $\beta-\mathrm{MnO}_{2}$. There might be a critical size of $\beta-\mathrm{MnO}_{2}$ which governs the phase transitions. The phase transition might be greatly reduced when the size of $\beta$ $\mathrm{MnO}_{2}$ is reduced to a certain level close to the mesoporous structure.

Apart from the application in lithium-ion batteries, $\beta-\mathrm{MnO}_{2}$ also found applications in supercapacitors and cathode catalyst for metal-air batteries. Similar to its application in the lithium-ion battery, the morphology of $\beta-\mathrm{MnO}_{2}$ can greatly affect its specific capacitance. Nanorod-shaped $\beta-\mathrm{MnO}_{2}$ exhibited a low specific capacitance around $28 \mathrm{~F} \cdot \mathrm{g}$ ${ }^{1 .}{ }^{17}$ However, ultrafine $\beta-\mathrm{MnO}_{2}$ nanowires with a diameter below $10 \mathrm{~nm}$ can reach a high specific capacitance close to $279 \mathrm{~F} \cdot \mathrm{g}^{-1} \cdot{ }^{18}$ The specific capacitance of ultrafine $\beta-\mathrm{MnO}_{2}$ can be further improved to $294 \mathrm{~F} \cdot \mathrm{g}^{-1}$ with the introduction of polypyrrole to form a composite. ${ }^{19}$ Moreover, two-dimensional $\beta-\mathrm{MnO}_{2}$ nanowire network showed even higher specific capacitance of $453.0 \mathrm{~F} \cdot \mathrm{g}^{-1}$ at a current density of $0.5 \mathrm{~A} \cdot \mathrm{g}^{-1} \cdot{ }^{20}$ And nanoflower shaped $\beta$ - 
$\mathrm{MnO}_{2}$ showed a reported capacitance as high as $296.3 \mathrm{~F} \cdot \mathrm{g}^{-1} \cdot{ }^{21}$ Recently, hybrid structures of $\beta-\mathrm{MnO}_{2}$ crystals were developed to further improve the electrochemical performance, including the hierarchical reduced graphene oxide $/ \beta-\mathrm{MnO}_{2}$ composite, ${ }^{22} \beta-\mathrm{MnO}_{2}$ nanosheets/nanowires core-shell structure, ${ }^{23}$ as well as $\beta-\mathrm{MnO}_{2} /$ birnessite core-shell structure. ${ }^{24}$ The incorporation of rGO improved the conductivity and offered higher surface areas for the cations adsorption. A high specific capacitance close to $362 \mathrm{~F} \cdot \mathrm{g}^{-1}$ was realized at a current density of $1.0 \mathrm{~A} \cdot \mathrm{g}^{-1} \cdot{ }^{22}$ The $\beta-\mathrm{MnO}_{2}$ nanosheets/nanowires offered disordered regions within the composite, which was proposed to be the reason for the increased specific capacitance near $190.5 \mathrm{~F} \cdot \mathrm{g}^{-1} \cdot{ }^{23} \mathrm{Zhu}$ et al further developed the $\beta-\mathrm{MnO}_{2} /$ birnessite core-shell structure based on a two-step hydrothermal synthesis process; the obtained composite delivered $657 \mathrm{~F} \cdot \mathrm{g}^{-1}$ specific capacitance in $1 \mathrm{M} \mathrm{Na} 2 \mathrm{SO}_{4}$ electrolyte. ${ }^{24}$ The improved electrochemical performance was related to the highly aligned birnessite nanosheets, which offers a large amount of active sites for cations adsorption and intercalation.

It can be concluded that the morphology (or exposed facets) of $\beta-\mathrm{MnO}_{2}$ greatly affected its electrochemical performance. However, the role of crystal facets in the application of $\beta-\mathrm{MnO}_{2}$ crystals in lithium-ion batteries and supercapacitors is still poorly understood so far. Although several groups have tried to develop the understanding this effect through computational simulation, ${ }^{25-28}$ experimental based investigations are still limited. This is partially because: (1) Facet-controlled synthesis of $\beta-\mathrm{MnO}_{2}$ is difficult to realize due to the thermodynamically preferred growth of the crystals along the tunnel direction to form 1D nanowires. (2) The identification of exposed crystal facets is 
challenging, which needs comprehensive characterizations combining X-ray diffraction,

Scanning electron microscopy, high-resolution transmission electron microscopy, as well as the cross-sectional analysis assisted with the ultramicrotomy technique.

\subsection{References}

1 Mizushima, K., Jones, P., Wiseman, P. \& Goodenough, J. B. $\operatorname{Li}_{\mathrm{x}} \mathrm{CoO}_{2}(0<\mathrm{x}<1)$ : A new cathode material for batteries of high energy density. Materials Research Bulletin 15, 783-789 (1980).

2 Antolini, E. $\mathrm{LiCoO}_{2}$ : formation, structure, lithium and oxygen nonstoichiometry, electrochemical behaviour and transport properties. Solid State Ionics 170, 159171 (2004).

3 Komaba, S., Yabuuchi, N. \& Kawamoto, Y. A new polymorph of layered $\mathrm{LiCoO}_{2}$. Chemistry Letters 38, 954-955 (2009).

4 Chen, H., Qiu, X., Zhu, W. \& Hagenmuller, P. Synthesis and high rate properties of nanoparticled lithium cobalt oxides as the cathode material for lithium-ion battery. Electrochemistry Communications 4, 488-491 (2002).

5 Liang, H. et al. Analysis of high rate performance of nanoparticled lithium cobalt oxides prepared in molten $\mathrm{KNO}_{3}$ for rechargeable lithium-ion batteries. Electrochemistry Communications 6, 789-794 (2004).

6 Liao, C.-L. \& Fung, K.-Z. Lithium cobalt oxide cathode film prepared by RF sputtering. Journal of Power Sources 128, 263-269 (2004).

7 Li, L., Meyer, W. H., Wegner, G. \& Wohlfahrt-Mehrens, M. Synthesis of submicrometer-sized electrochemically active lithium cobalt oxide via a polymer precursor. Advanced Materials 17, 984-988 (2005).

8 Amatucci, G., Tarascon, J. \& Klein, L. $\mathrm{CoO}_{2}$, the end member of the $\mathrm{Li}_{\mathrm{x}} \mathrm{CoO}_{2}$ solid solution. Journal of The Electrochemical Society 143, 1114-1123 (1996).

9 Moradpour, A. et al. Resistive switching phenomena in $\mathrm{Li}_{\mathrm{x}} \mathrm{CoO}_{2}$ thin films. Advanced Materials 23, 4141-4145 (2011).

10 Zhu, X. et al. Direct observation of lithium-ion transport under an electrical field in $\mathrm{Li}_{\mathrm{x}} \mathrm{CoO}_{2}$ nanograins. Scientific Reports 3, 1084 (2013). 
11 Tang, W., Yang, X., Liu, Z. \& Ooi, K. Preparation of $\beta-\mathrm{MnO}_{2}$ nanocrystal/acetylene black composites for lithium batteries. Journal of Materials Chemistry 13, 2989-2995 (2003).

12 Murphy, D., Di Salvo, F., Carides, J. \& Waszczak, J. Topochemical reactions of rutile related structures with lithium. Materials Research Bulletin 13, 1395-1402 (1978).

13 Luo, J.-Y., Zhang, J.-J. \& Xia, Y.-Y. Highly electrochemical reaction of lithium in the ordered mesoporosus $\beta-\mathrm{MnO}_{2}$. Chemistry of Materials 18, 5618-5623 (2006).

14 Huang, X. et al. Highly crystalline macroporous $\beta-\mathrm{MnO}_{2}$ : hydrothermal synthesis and application in lithium battery. Electrochimical Acta 55, 4915-4920 (2010).

15 Chen, W.-M. et al. Controllable synthesis of hollow bipyramid $\beta-\mathrm{MnO}_{2}$ and its high electrochemical performance for lithium storage. ACS Applied Material \& Interfaces 4, 3047-3053 (2012).

16 Zhan, D., Zhang, Q., Hu, X. \& Peng, T. Single-crystal $\beta-\mathrm{MnO}_{2}$ hollow bipyramids: synthesis and application in lithium ion batteries. RSC Advances $\mathbf{3}$, 5141-5147 (2013).

17 Ghodbane, O., Pascal, J.-L. \& Favier, F. Microstructural effects on charge-storage properties in $\mathrm{MnO}_{2}$-based electrochemical supercapacitors. ACS Applied Material \& Interfaces 1, 1130-1139 (2009).

18 Jiang, H., Zhao, T., Ma, J., Yan, C. \& Li, C. Ultrafine manganese dioxide nanowire network for high-performance supercapacitors. Chemical Communication 47, 1264-1266 (2011).

19 Zang, J. \& Li, X. In situ synthesis of ultrafine $\beta-\mathrm{MnO}_{2} /$ polypyrrole nanorod composites for high-performance supercapacitors. Journal of Materials Chemistry 21, 10965-10969 (2011).

20 Wei, C. et al. Two-dimensional $\beta-\mathrm{MnO}_{2}$ nanowire network with enhanced electrochemical capacitance. Scientific Report 3 (2013).

21 Yu, L.-L., Zhu, J.-J. \& Zhao, J.-T. Beta-manganese dioxide nanoflowers selfassembled by ultrathin nanoplates with enhanced supercapacitive performance. Journal of Materials Chemistry A 2, 9353-9360 (2014).

22 Zhu, S. et al. Self-assembled three-dimensional hierarchical graphene hybrid hydrogels with ultrathin $\beta-\mathrm{MnO}_{2}$ nanobelts for high performance supercapacitors. Journal of Materials Chemistry A 3, 1540-1548 (2015). 
23 Shao, J. et al. Mechanism analysis of the capacitance contributions and ultralong cycling-stability of the isomorphous $\mathrm{MnO}_{2} @ \mathrm{MnO}_{2}$ core/shell nanostructures for supercapacitors. Journal of Materials Chemistry A 3, 6168-6176 (2015).

24 Zhu, S. et al. Structural directed growth of ultrathin parallel birnessite on $\beta-\mathrm{MnO}_{2}$ for high-performance asymmetric supercapacitors. ACS Nano 12, 1033-1042 (2018).

25 Tompsett, D. A., Parker, S. C., Bruce, P. G. \& Islam, M. S. Nanostructuring of $\beta$ $\mathrm{MnO}_{2}$ : The important role of surface to bulk ion migration. Chemistry of Materials 25, 536-541 (2013).

26 Wang, D. et al. $\beta-\mathrm{MnO}_{2}$ as a cathode material for lithium ion batteries from first principles calculations. PCCP 15, 9075-9083 (2013).

27 Tompsett, D. A., Parker, S. C. \& Islam, M. S. Rutile ( $\beta$ - $) \mathrm{MnO}_{2}$ surfaces and vacancy formation for high electrochemical and catalytic performance. Journal of the American Chemical Society 136, 1418-1426 (2014).

28 Dawson, J. A. \& Tanaka, I. Li intercalation into a $\beta-\mathrm{MnO}_{2}$ grain boundary. $A C S$ Applied Material \& Interfaces 7, 8125-8131 (2015). 


\section{Chapter 2 Experimental Methods}

This chapter summarizes the main experimental techniques used in the Ph.D. projects, including conductive atomic force microscopy, hydrothermal synthesis, materials characterization techniques (XRD, SEM, TEM, STEM/EELS), as well as electrochemical tests.

\subsection{Conductive atomic force microscopy (C-AFM)}

C-AFM was realized on a Bruker Veeco Dimension Icon system with the use of their TUNA external module. Multi75E-G conductive AFM probes with Pt/Ir coatings were used. The spring constant of the probe and the deflection sensitivity of the system were calibrated before running the C-AFM test. Calibration of the spring constant was based on the Sader method. The spring constant was calculated based on the dimension of the cantilever, the measured resonance frequency, and Q factor. The specific value of the resonance frequency and $\mathrm{Q}$ factor were obtained under tapping mode beforehand. Deflection sensitivity of the system was evaluated on a sapphire sample to reduce surface deformation.

\subsection{Hydrothermal synthesis of $\beta-\mathrm{MnO}_{2}$}

Hydrothermal synthesis is commonly used for the growth of $1 \mathrm{D} \mathrm{MnO}_{2}$ single crystal structures. Two different kinds of morphologies, nanowire and microrod, were adopted to study the lateral surface evolution in the $\beta-\mathrm{MnO}_{2}$ crystals. The synthesis of $\beta-\mathrm{MnO}_{2}$ nanowires was based on the redox reaction between $\left(\mathrm{NH}_{4}\right)_{2} \mathrm{~S}_{2} \mathrm{O}_{8}$ and $\mathrm{MnSO}_{4} \cdot \mathrm{H}_{2} \mathrm{O}$. The crystal structure was obtained from aqueous reactions in sealed Teflon-lined steel autoclave 
under a reaction temperature of $140{ }^{\circ} \mathrm{C}$ for different times $(12,18,24 \mathrm{~h})$. The $\beta-\mathrm{MnO}_{2}$ microrod was obtained from a redox reaction between $\mathrm{KMnO}_{4}$ and $\mathrm{MnCl}_{2} \cdot 4 \mathrm{H}_{2} \mathrm{O}$ under a reaction temperature of $180^{\circ} \mathrm{C}$ for different hours $(12,24,48 \mathrm{~h})$. All the samples were collected by centrifugation, followed by consecutively cleaning with deionized water and ethyl alcohol, and finally dried in air at $60^{\circ} \mathrm{C}$ for $12 \mathrm{~h}$.

Facet-controlled synthesis of $\beta-\mathrm{MnO}_{2}$ was realized through controlling the amount of $\mathrm{KCl}$ added to the redox reaction between $\mathrm{KMnO}_{4}$ and $\mathrm{MnCl}_{2} \cdot 4 \mathrm{H}_{2} \mathrm{O}$. The amount of $\mathrm{KCl}$ was adjusted between $0.02 \mathrm{M}$ to $0.09 \mathrm{M}$ to realize a morphology change from bipyramid prism to octahedra.

\subsection{Materials characterization techniques}

To examine the phase and morphology of the obtained materials, several characterization techniques were used, including Powder X-ray diffraction (XRD), Field-Emission Scanning Electron Microscopy (FE-SEM), High-resolution Transmission Electron Microscopy (HR-TEM), as well as Scanning Transmission Electron Microscopy (STEM) with Electron Energy Loss Spectroscopy (EELS) and Energy-dispersive X-ray Spectroscopy (EDS) capabilities.

XRD patterns of the materials were acquired on a Scintag XDS2000 powder X-ray diffractometer with $\mathrm{Cu} \mathrm{K} \alpha$ radiation. The morphology of the $\beta-\mathrm{MnO}_{2}$ crystals was examined using a Hitachi S-4700 FE-SEM. Internal tunnel structures and growth directions of the $\beta-\mathrm{MnO}_{2}$ crystals were further identified combing JEOL 3010 HRTEM, aberrationcorrected JEOL JEM-ARM 200CF STEM/EELS. Cross-section samples of the $\beta-\mathrm{MnO}_{2}$ 
crystals were prepared by ultramicrotomy (Leica Ultracut UCT) to identify the crystal facets of the structures and examine the internal tunnel structures.

\subsection{Electrochemical performance tests}

To study the effect of crystal facets on the electrochemical performance, different morphologies of $\beta-\mathrm{MnO}_{2}$ were applied as the cathode for lithium-ion batteries, electrode materials for symmetric supercapacitors, as well as the cathode catalyst for lithium-air batteries.

The lithium-ion battery and supercapacitor tests were performed on a Bio-Logic VMP3 electrochemical workstation. The lithium-ion battery cathode was prepared by mixing synthesized $\beta-\mathrm{MnO}_{2}$ crystals, super $\mathrm{P}$, and polyvinylidene difluoride (PVDF) with N-methylpyrrolidone (NMP) solvent. PVDF was first dissolved in the NMP solution under constant stirring for $30 \mathrm{~min}$. After the PVDF was completely dissolved, super P was added and stirred for 2 hours to ensure well dispersion of the carbon in the solvent. The active material was added at last and stirred for another 2 hours. The obtained cathode slurry shall have well-distributed active materials and super P in the solvent. The fluidity of the slurry shall be adjusted by the ratio between the amount of active material and the volume of the NMP solvent (around 500 $\mu \mathrm{L}$ NMP was used for the dissolve of $100 \mathrm{mg}$ of active material).

The slurry should have enough fluidity for the following casting on the aluminum foil, but also not too diluted to cause precipitation of the active materials or super P. The thickness of the electrode was controlled at $90 \mu \mathrm{m}$. Standard CR2032 coin cells were assembled in Ar-filled MBraun glove box with oxygen and water level below $0.5 \mathrm{ppm}$. The amount of 
$\mathrm{LiPF}_{6}$ electrolyte was also controlled at $100 \mu \mathrm{L}$. The coin cells were assembled with lithium metal as the anode and glass fiber as the separator. Galvanostatic charge-discharge was performed with a potential range between $2.0 \mathrm{~V}$ and $4.2 \mathrm{~V}$ vs. $\mathrm{Li} / \mathrm{Li}^{+}$. Electrochemical impedance spectroscopy (EIS) was performed with a $10 \mathrm{mV}$ AC amplitude and a frequency range from $10 \mathrm{mHz}$ to $100 \mathrm{kHz}$.

The electrode for supercapacitor application was fabricated following the similar procedure except casting on a carbon paper substrate instead of aluminum foil. Symmetric supercapacitor was assembled using CR2032 coin cells with two similar $\beta-\mathrm{MnO}_{2}$ electrodes, glass fiber separator, and $1 \mathrm{M} \mathrm{Na}_{2} \mathrm{SO}_{4}$ liquid electrolyte. Standard CV curves and charge-discharge curves were obtained within the voltage range of $0 \sim 0.8 \mathrm{~V}$ under different scan rate and current density, respectively. All the electrochemical tests were performed at room temperature. 


\section{Chapter 3 Localized mechanical stress induced ionic redistribution in a layered $\mathrm{LiCoO}_{2}$ cathode $^{1}$}

Controlling the transport of ions within electrodes is highly desirable for the operation of rechargeable ion batteries. Here, for the first time, we report the role of mechanical stress in controlling the redistribution of lithium ions in a layered $\mathrm{LiCoO}_{2}$ electrode at a resolution of $\sim 100 \mathrm{~nm}$. Under a higher stress field, more active redistribution of lithium ions was observed along the grain boundaries than the interiors of the layered $\mathrm{LiCoO}_{2}$. The dynamic force ramping test proved the external stress field $(<100 \mathrm{nN})$ is capable of inducing the resistive-switching effect of the layered $\mathrm{LiCoO}_{2}$. The comparison test on the highly ordered pyrolytic graphite (HOPG) substrate further demonstrated the improved current responses from the layered $\mathrm{LiCoO}_{2}$ resulted from the deficiency of lithium ions, rather than the increase of tip-sample contact area. Our findings will pave the road for a full understanding of how mechanical stimulus can affect the distribution of ions in the layered electrodes of rechargeable ion batteries.

\subsection{Introduction}

Lithium-ion batteries have been widely used as power sources for portable electronic devices as well as hybrid vehicles. ${ }^{1-3}$ To meet the future demand for energy storage, improvements in charge capacity and power density are still needed. ${ }^{4}$ Nanostructured electrode materials have shown promising potential in improving the performance of

\footnotetext{
${ }^{1}$ The material contained in this chapter was previously published in ACS Applied Materials \& Interfaces. (Reprinted with permission from W. Yao, F. Long, R. S. Yassar. ACS Appl. Mater. Interfaces, 2016, 8 (43), pp 29391-29399. Copyright 2016, American Chemical Society.)
} 
lithium-ion batteries, because they offer higher contact area, increased lithium intercalation rate, and shorter electron pathways. ${ }^{5}$ To improve the understanding of the relationship between the nanostructures and their electrochemical performance, nanoscale studies are needed with the help of high-resolution microscopes. ${ }^{6-9}$

Layered $\mathrm{LiCoO}_{2}$ is the most widely used and commercialized cathode material for lithium-ion batteries due to its relatively high capacity and excellent stability. ${ }^{10,11}$ Under a concentrated electric field, the lithium ions tend to be driven away from the slabs of $\mathrm{CoO}_{2}$ octahedral structures, resulting in localized deficiency of lithium ions. ${ }^{8}$ The deficiency of lithium ions further induces two major changes inside the $\mathrm{LiCoO}_{2}$ electrode: lattice expansion along its $c$-axis and resistive switching from semi-conductive to metallic state. ${ }^{12-}$

${ }^{14}$ The lattice expansions along the $c$-axis $\left(\mathrm{Li}_{\mathrm{x}} \mathrm{CoO}_{2}, 0.5<\mathrm{x}<1\right)^{15}$ are detectable using atomic force microscopy (AFM). Based on this dimensional change, Balke, et al successfully developed electrochemical strain microscopy (ESM) to study the distribution of lithium ions inside layered $\mathrm{LiCoO}_{2}$ under both direct and alternating bias voltages. ${ }^{8}$ The resistive switching phenomenon is related to the electromigration of ions and electrochemical reactions. This phenomenon was also observed with other cations $\left(\mathrm{Cu}^{2+}\right.$, $\mathrm{Ag}^{+}$or $\left.\mathrm{Ni}^{2+}\right)^{16-18}$ and substrates ${ }^{19}$ other than layered $\mathrm{LiCoO}_{2}$. Using conductive $\mathrm{AFM}(\mathrm{C}-$ AFM), the resistive switching effect can be revealed based on the current responses from the substrate under a bias voltage. The obtained current mapping image can be used to visualize the redistribution of lithium ions. Using C-AFM, Zhu, et al proved more active redistribution of lithium ions along $\mathrm{LiCoO}_{2}$ grain boundaries than the interiors and further demonstrated the energy barrier for the diffusion of lithium ions is lower along the 
boundaries. ${ }^{20}$ Other influencing factors for the redistribution of lithium ions inside the layered $\mathrm{LiCoO}_{2}$ cathode, such as temperature ${ }^{21}$ and grain orientation ${ }^{22}$, have been studied at the nanoscale using ESM.

Under a positive electric field, the internal mechanical strain is generated as a result of the expansion along the $c$-axis of the layered $\mathrm{LiCoO}_{2}$ cathode because of the deficiency of lithium ions. This is similar to the piezoelectric effect, where the applied electric field can generate internal mechanical strains. This process is mainly reversible for piezoelectric materials. However, for layered $\mathrm{LiCoO}_{2}$, whether external mechanical stress can regulate the redistribution of ions has never been studied. In this report, commercial layered $\mathrm{LiCoO}_{2}$ substrate was used to study the effect of localized stresses on the redistribution of lithium ions under a constant electric field. High-resolution tunneling current module (TUNA) was adopted for C-AFM tests to reach a high current resolution $(<1 \mathrm{pA})$. The effect of external stresses on the resistive switching of layered $\mathrm{LiCoO}_{2}$ was also studied by a dynamic force ramping technique.

\subsection{Methods}

3.2.1 Materials. The $\mathrm{LiCoO}_{2}$ thin film was purchased from MTI Corp and used as received. The thickness of the film was $0.1 \mathrm{~mm}$ and coated on an aluminum foil. The scanning electron microscopy (SEM) image was obtained from a Hitachi S-4700 Field Emission scanning microscope under an accelerating voltage of $10 \mathrm{kV}$. X-ray powder diffraction (XRD) pattern was obtained from a Scintag XRS2000 powder diffractometer using $\mathrm{Cu} \mathrm{K} \alpha_{1}$ radiation with a step size of $0.02^{\circ}$. The HOPG sample was obtained from the 
standard sample kit offered from Bruker Company. The thickness of the HOPG sample is around $2 \mathrm{~mm}$ and mounted on a steel plate with a conductive polymer.

3.2.2 C-AFM test. All C-AFM tests were carried out using the Dimension ICON system (Bruker, CA.) under ambient conditions. Conductive AFM probes (Multi75E-G, budget sensors) with double-sided Pt/Ir coating were used. The length and width of the probe cantilever are $225 \mu \mathrm{m}$ and $28 \mu \mathrm{m}$, respectively. The TUNA external module (Figure 3-1a) was used to provide high current sensitivity. The offset and gain adjustments were performed for the TUNA module before the C-AFM tests. Specific 100 Mega Ohms dummy resistor was used for the gain adjustment. The current sensitivity used was set to $100 \mathrm{nA} / \mathrm{V}$ during the tests. The bias voltage was added to the substrate as illustrated in Figure 1a. Under the C-AFM mode, the AFM probe was kept in contact with the substrate to obtain the current response.

3.2.3 Calibration of deflection sensitivity and spring constant. For the calibration of deflection sensitivity, a sapphire sample (Young's modulus $345 \mathrm{GPa}$, from the standard AFM sample kit) was used to ensure enough stiffness and reduce the influence of surface deformation. Average deflection sensitivity of the system was obtained from sensitivity values generated from five different spots on the sapphire sample. The sensitivity test was repeated three times at each spot. Spring constants of the probes were calibrated using the Sader method. ${ }^{23}$ The spring constant is determined by its resonance frequency, $\mathrm{Q}$ factor, and geometry. The value of resonance frequency and Q factor were obtained under the tapping mode before the C-AFM test. 
3.2.4 Dynamic force ramping. The dynamic force ramping was realized by ramping the distance $(\boldsymbol{Z})$ between the substrate and the cantilever rest position instead. Under C-AFM mode, the $\boldsymbol{Z}$ value was ramped with a ramp size of $250 \mathrm{~nm}$ and a ramp rate of $0.3 \mathrm{~Hz}$. The forward and retract velocities of the probe were kept the same at $145 \mathrm{~nm} / \mathrm{s}$. Once the relationship between $\boldsymbol{Z}$ value and current response was obtained, the $\boldsymbol{Z}$ value can be transformed into its corresponding applied force by using the force-distance curves generated on the substrate. Under contact mode, the tip-sample distance equals zero, thus the distance between the substrate and the cantilever rest position $(\boldsymbol{Z})$ is equal to the deflection of the cantilever, which is proportional to the force applied on the substrate according to the Hooke's Law.

3.2.5 Forces calibration. Adjusting the forces applied through the AFM probe controls the mechanical stress applied to the substrate. Under the C-AFM mode, the probe stays in contact with the substrate. Actual forces applied on the substrate can be calibrated based on the deflection of the cantilever according to the Hook's law. ${ }^{24}$ The cantilever deflection can be calculated based on the setpoint value and the deflection sensitivity of the probe. The applied force can be determined by multiplying the value of setpoint $(\mathrm{V})$, the deflection sensitivity of the system $(\mathrm{nm} / \mathrm{V})$, and the spring constant of the probe $(\mathrm{N} / \mathrm{m})$. For the probes used on different substrates, the calibrated parameters are shown in Table 1. Assuming the deflection sensitivity of the system and spring constant of the probe were constant during the test, the applied forces can be considered as proportional to the setpoint value. Thus, the stress loading process can be realized by increasing the setpoint value from 0.1 to 0.8 $\mathrm{V}$ with a step size of $0.1 \mathrm{~V}$. The unloading process was conducted with the same step size 
but changing the setpoint value from 0.8 to $0.1 \mathrm{~V}$. The setpoint value was limited to $0.8 \mathrm{~V}$ to prevent the probe from damaging the substrate.

Table 1 Calibrated parameters of the AFM probes used on different substrates

\begin{tabular}{cccc}
\hline \multirow{2}{*}{ Substrate } & $\begin{array}{c}\text { Deflection sensitivity } \\
(\mathrm{nm} / \mathrm{V})\end{array}$ & $\begin{array}{c}\text { Pesonance } \\
\text { frequency }(\mathrm{kHz})\end{array}$ & $\begin{array}{c}\text { Spring constant } \\
(\mathrm{N} / \mathrm{m})\end{array}$ \\
\hline $\mathrm{LiCoO} 2$ & $117.5 \pm 3.2$ & 66.87 & $2.24 \pm 0.05$ \\
$\mathrm{HOPG}$ & $107.4 \pm 0.5$ & 73.95 & $2.48 \pm 0.05$ \\
\hline
\end{tabular}

\subsection{Results and Discussion}

3.3.1 C-AFM tests on the layered $\mathrm{LiCoO}_{2}$ cathode. The topography image in Figure 3$1 \mathrm{~b}$ and SEM image (See Supporting Information, Figure S3-1) show a layered and polycrystalline structure of the $\mathrm{LiCoO}_{2}$ thin film. The XRD diffraction peaks (Figure S32) are in good agreement with rhomb-centered $\mathrm{O}_{3}-\mathrm{LiCoO}_{2}$ (JCPDS 16-0427). Minor peaks at $37.4^{\circ}$ and $44.4^{\circ}$ can be indexed to $\mathrm{Co}_{3} \mathrm{O}_{4}$ (JCPDS 80-1544), which shall come from the manufacturing process. The peak intensity ratio also fits well with the standard, indicating no preferred growth direction for the $\mathrm{LiCoO}_{2}$ substrate. The applied DC voltage was selected at $2 \mathrm{~V}$ to ensure the resistive switching effect can be generated. ${ }^{25}$ Electric current mapping under different localized stress is shown in Figure 3-1c. The upper row in Figure 1c shows the stress loading process (setpoint at $0.1,0.3,0.5$, and $0.7 \mathrm{~V}$, respectively), while the lower row shows the stress unloading process. The bright areas in the electric current mapping image indicate conductive regions on the substrate. During the stress loading process, under an applied force of $26.3 \pm 1.1 \mathrm{nN}$ (setpoint at $0.1 \mathrm{~V}$ ), a low electric current contrast was observed between the boundaries and the grain interiors. Further increasing 
the applied force to $78.9 \pm 3.2 \mathrm{nN}$ (setpoint at $0.3 \mathrm{~V}$ ) caused the conductive regions to expand and these conductive regions mainly distributed along the grain boundaries. During the unloading process, the area of conductive regions and the intensity of electric current output reduced along with the decrease of applied stress. The electric current responses under the $26.3 \pm 1.1 \mathrm{nN}$ and the $78.9 \pm 3.2 \mathrm{nN}$ applied forces were slightly higher than that during the loading process. This is related to the hysteresis effect from the loading process. $^{26}$

Under the DC voltage of $2 \mathrm{~V}$, some areas of the substrate were still in a nonconductive state. This indicated that the lithium ions were not completely driven away by the external electric field. The resistive switching of $\mathrm{Li}_{\mathrm{x}} \mathrm{CoO}_{2}$ happens when the lithium composition is in the range of $0.75 \leq \mathrm{x} \leq 0.94 .^{12}$ The observed electric current distribution along grain boundaries is consistent with the work from Zhu et al ${ }^{20}$. Instead of even distribution of current response along all boundary areas, some boundary areas did not show electric current responses in our case. This is due to the polycrystalline structure of the $\mathrm{LiCoO}_{2}$ substrate; different grains are oriented in different directions. The diffusion of lithium ions is only preferred along the (001) planes, between the adjacent $\mathrm{CoO}_{2}$ layers. ${ }^{8}$ The redistribution of lithium ions is maximized when the orientation of the layered structure is parallel to the external electric field, because of the high mobility of the lithium ions and possible surface reactions. ${ }^{22}$ When the layered $\mathrm{Li}$-ion $/ \mathrm{CoO}_{2}$ planes are aligned normally to the electric field, the redistribution of lithium ions would be minimized, but strong out-of-plane volume change along the $c$-axis can be observed. Because of the different orientations of the layered $\mathrm{LiCoO}_{2}$ grains, dissimilar electrical current responses 
can be detected at the boundaries (Figure S3-3). In our case, the AFM scan direction and sample position were kept the same all the time, so grain orientations did not affect our comparison among different electrical current signals.

a

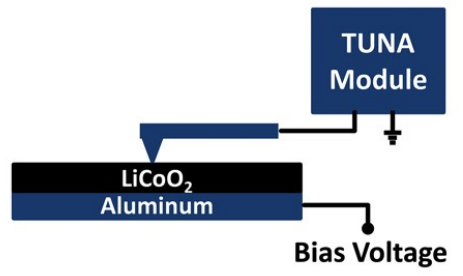

C

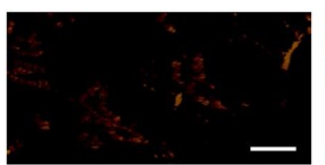

$26.3 \pm 1.1 \mathrm{nN}$

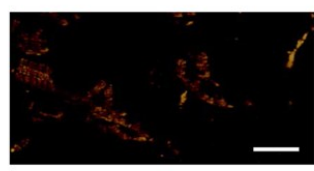

$26.3 \pm 1.1 \mathrm{nN}$

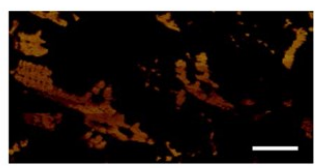

$78.9 \pm 3.2 \mathrm{nN}$

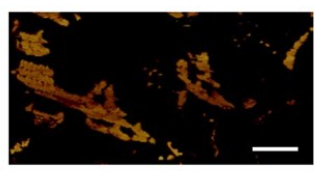

$78.9 \pm 3.2 \mathrm{nN}$

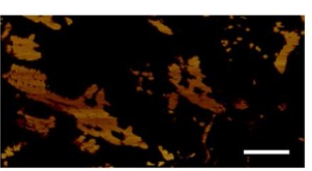

$131.6 \pm 5.4 \mathrm{nN}$

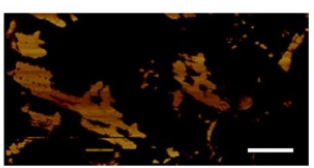

$131.6 \pm 5.4 \mathrm{nN}$

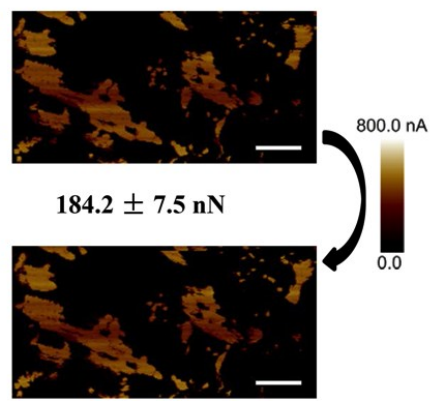

$184.2 \pm 7.5 \mathrm{nN}$

Figure 3-1 (a) A schematic image of the C-AFM test setup with TUNA module. (b) Height (left) and deflection (right) images of the $\mathrm{LiCoO}_{2}$ substrate. The dashed line (e-e') is for the section analysis discussed in Figure 2b. (c) Electric current mapping of the corresponding area during the stress loading (upper row) and unloading processes (lower row) under $2 \mathrm{~V}$ bias voltage. Scale bars are $300 \mathrm{~nm}$.

For quantitative analysis of the current response, arithmetic mean roughness $\left(R_{a}\right)$ and the root-mean-square roughness $\left(\mathrm{R}_{\mathrm{q}}\right)$ of the current signal were analyzed. Under the bias voltage of $2 \mathrm{~V}$, the $\mathrm{R}_{\mathrm{a}}$ value increased nonlinearly from 43.6 to $183.0 \mathrm{nA}$ when the 
external force was increased from $26.3 \mathrm{nN}$ to $210.5 \mathrm{nN}$ (Figure 3-2a). For a better understanding of the effect of stress field on Li-ion redistribution, C-AFM tests without bias voltages were also performed at the same time under the same loading/unloading conditions (Figure S3-4). To reduce any possible hysteresis effect from the electric field, C-AFM tests without the bias voltage were performed before the tests with the $2 \mathrm{~V}$ DC voltage. When the electric field is removed $(0 \mathrm{~V})$, the $\mathrm{R}_{\mathrm{a}}$ value increased from 0.0135 to $0.0254 \mathrm{nA}$ under the same loading condition. The current observed from the regions with Li-ion deficiency was negative, which was due to the negatively charged $\mathrm{CoO}_{2}$ layers after the interlayer lithium ions were driven away by external stresses. The detected current flow maybe was induced by the potential differences between the negatively charged $\mathrm{CoO}_{2}$ layers and the redistributed lithium ions. This demonstrated that mechanical stress is capable of inducing lithium ions redistribution with or without the electric field. The similar trend was observed with the change of the $\mathrm{R}_{\mathrm{q}}$ value versus external stress (Figure $\mathrm{S} 3-5)$. The increase of $\mathrm{R}_{\mathrm{a}}$ and $\mathrm{R}_{\mathrm{q}}$ values indicate more conductive regions were induced by the enhanced external stress. The average value of the current output is identical during both loading and unloading processes, indicating a relatively reversible effect of the external stress field on the current response at the level of a few hundred nano-newtons. The increasing rate of the $\mathrm{R}_{\mathrm{a}}$ value reduced along with the improving of applied forces. The decrease of this rate indicates the slowing down of the distribution of lithium ions. It is possible that this stress-induced lithium ion redistribution may be limited to some extent. The effect of external stress on redistribution of lithium ions was weakened with the depletion of lithium ions inside its layered structure. 
Based on the cross-section analysis results (Figure 3-2b), when the applied external stress field was increased, an expansion of the conductive regions was observed, indicating larger areas of the layered $\mathrm{LiCoO}_{2}$ switched from a semi-conductive state to a metallic state. The sharp current peaks along the conductive regions are consistent with the resistive switching phenomena of the layered $\mathrm{LiCoO}_{2}$ cathode. The two dashed lines g and g' were drawn to show the change of current response inside the conductive region along with the increase of the external stresses. Clearly, the magnitude of the electric current response increased inside the g-g' region. This demonstrated that adding more stresses increased the redistribution of lithium ions inside the conductive regions.
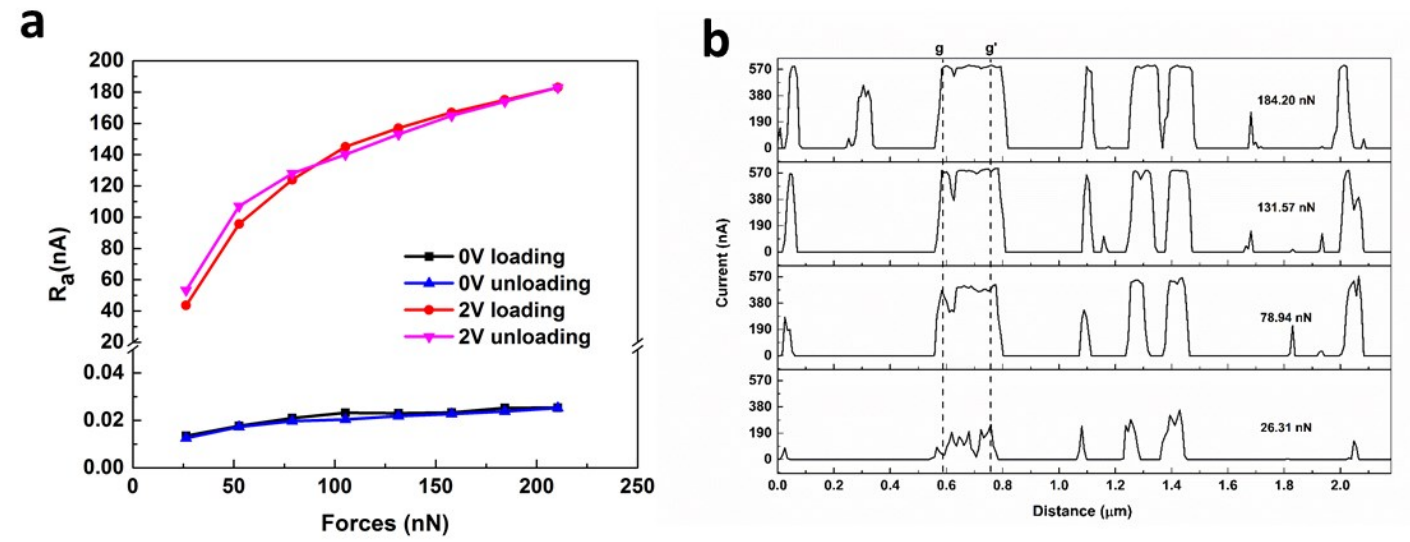

Figure 3-2 (a) Arithmetic mean roughness $\left(\mathrm{R}_{\mathrm{a}}\right)$ of electric current output from the $\mathrm{LiCoO}_{2}$ thin film as a function of applied forces under $0 \mathrm{~V}$ and $2 \mathrm{~V}$ bias voltage. (b) Cross section along the diagonal line e-e' in Figure $1 \mathrm{~b}$ for the current signals during the stress loading process. Dashed lines g and g' were drawn for comparison of the current signals from the same location. 
To compare the scan rate with the diffusion rate of lithium ions, further experiments were conducted. The scan rate used for obtaining C-AFM images (Figure 3-1c) was 3.2 $\mu \mathrm{m} / \mathrm{s}(0.8 \mathrm{~Hz})$. Three C-AFM images (Figure S3-6) were obtained consecutively at the same location under the same scan rate of $3.2 \mu \mathrm{m} / \mathrm{s}(0.8 \mathrm{~Hz})$ and the same bias voltage of $2 \mathrm{~V}$. Figure S3-5b was obtained immediately after Figure S3-5a under the same condition. No obvious contrast change was observed, indicating fast and stable current response from the substrate. Figure S3-5c was obtained soon after Figure S3-5b with an increase in applied force to $52.6 \mathrm{nN}$ while keeping other parameters the same. When the applied force was increased, a higher contrast was observed immediately. This demonstrates that the redistribution of lithium ions is faster enough than our scan rate, and thus will not affect our current mapping.

3.3.2 Dynamic force ramping on the layered $\mathrm{LiCoO}_{2}$. To further study the influence of external stress field on the redistribution of lithium ions at different parts of the layered $\mathrm{LiCoO}_{2}$ cathode, the dynamic force ramping was performed and representative forcecurrent curves were obtained from three different spots on the $\mathrm{LiCoO}_{2}$ substrate, as shown in Figure 3-3a. Spot 1 is located in the interior of the $\mathrm{LiCoO}_{2}$ grains, while spots 2 and 3 are located along different grain boundaries. 

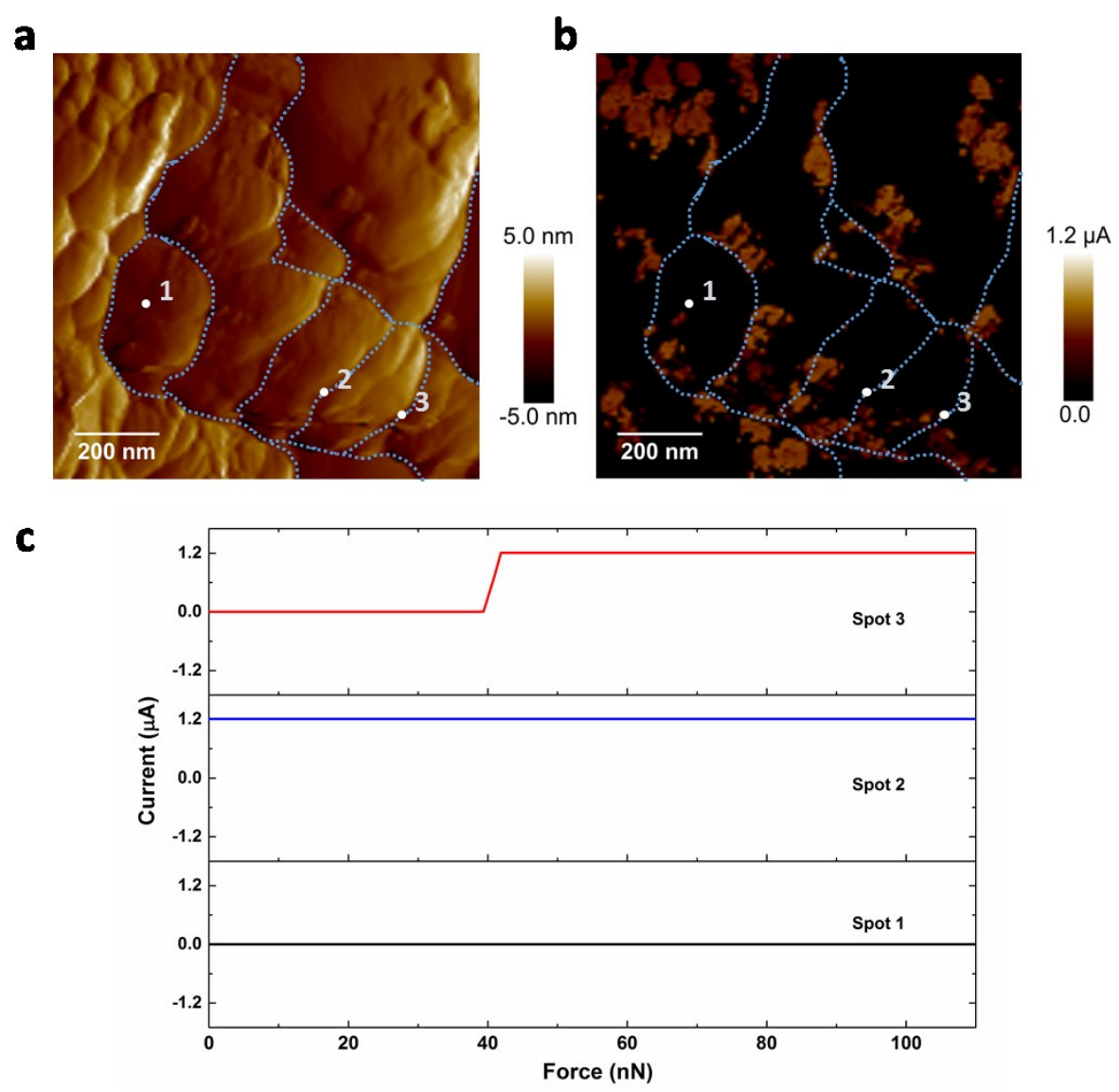

Figure 3-3 Deflection (a) and TUNA current output images (b) obtained on the $\mathrm{LiCoO}_{2}$ substrate under a bias voltage of $2 \mathrm{~V}$. Blue dashed lines were drawn to indicate different grain boundaries. (c) The representative force-current curves obtained from three different spots $(1,2$, and 3 in Figure $3 a)$ respectively.

The same kind of conductive probes were used for dynamic force ramping tests. The calibrated deflection sensitivity was $108.9 \pm 2.0 \mathrm{~nm} / \mathrm{V}$ and the calibrated spring constant was $2.65 \mathrm{~N} / \mathrm{m}$. Obtained relationships between applied forces and current responses are shown in Figure 3c. The electric current map in Figure $3 \mathrm{~b}$ shows a positive current output along the grain boundaries (spots 2 and 3). The force applied to obtain Figure 3-3b was $28.8 \pm 2.0 \mathrm{nN}$. Although the ramp size was $250 \mathrm{~nm}$, forces applied on the $\mathrm{LiCoO}_{2}$ 
substrate were only effective in the range of $0 \sim 110 \mathrm{nN}$ (Figure 3-3c). The current responses detected during the tip retracting were used to study their relationship with the applied forces.

Spot 1 was located inside the grain interiors and showed an insulator behavior, indicating a reluctant redistribution of lithium ions inside the grain interiors. The behavior of spot 1 is consistent with the nonconductive regions in Figure 3-2b ("valleys" of the graph). Spots 2 and 3 were located along the layered grain boundaries. Spot 2 showed a pure conductive behavior, indicating a high deficiency of lithium ions inside this region, which is consistent with the conductive regions in Figure 3-2b ("peaks" of the graph). The $1.2 \mu \mathrm{A}$ platform is actually the detection limit of the TUNA module. Spot 3 switched from a semi-conductive state to a conductive state when the applied force reached around $40 \mathrm{nN}$. This region remained conductive when the applied force was further increased up to 110 $\mathrm{nN}$. This conductivity change was shown to be reversible during the tip trace and retrace process (Figure S3-7). This behavior is also consistent with the step-by-step stress loadingunloading condition, indicating reversible resistive switching induced by external stresses.

The change of current response versus applied stresses at spot 3 looks different from the trend observed in Figure 3-2a, where a slight increase was observed instead of a sharp resistive switching peak. This is because Figure 3-2a offers the variation of averaged current response $\left(\mathrm{R}_{\mathrm{a}}\right)$ from a scanning area of $2 \mu \mathrm{m}^{2}$ instead of individual spots. By adding more stresses, a larger region of the $\mathrm{LiCoO}_{2}$ substrate will switch to the conductive state, which will result in an increase in the averaged current value. But the conductive spots may remain conductive and give the same current response even under higher stress field. 
During the step-by-step loading condition, the detection limit $(1.2 \mu \mathrm{A})$ was not reached over $110 \mathrm{nN}$. This may be related to the different grain orientations because the two tests were conducted in different areas. On the other hand, different loading methods were used. For the dynamic loading condition, other than the energy from the applied stresses, the kinetic energy of the probe will also accumulate, get absorbed by the substrate and further facilitate the redistribution of lithium ions.

To reveal the resistance switching effect of the layered $\mathrm{LiCoO}_{2}$ substrate, representative $\mathrm{I} / \mathrm{V}$ curves from the grain interiors and boundaries were also acquired (Figure S3-8). The grain interiors showed clearly a semiconductor behavior (Figure S3$8 \mathrm{~b}$ ), and higher threshold voltage was shown at the spot further away from the boundaries. The grain boundaries, however, showed a non-ohmic conductor behavior, indicating lowest energy barrier for the diffusion of lithium ions (Figure S3-8c).

In sum, the applied external stress $(<100 \mathrm{nN})$ is able to induce reversible redistribution of lithium ions under certain electric fields. Comparing the current output at spot 1 with that at spots 2 and 3, the grain boundaries show more active redistribution of lithium ions than the grain interiors. The result is identical with previous studies that the Li-ion diffusion barrier along the grain boundaries is $0.7 \mathrm{eV}$, which is relatively low compared with diffusion barrier of $6.8 \mathrm{eV}$ in the interiors. ${ }^{20}$

3.3.3 Comparison C-AFM test on the HOPG. From the electric current mapping on the layered $\mathrm{LiCoO}_{2}$, higher current responses were observed under higher applied stresses. However, with the increase of the applied stresses, the tip-sample contact area would also 
increase, which could reduce the tip-sample contact resistance ${ }^{27}$ and further increase the current output. To determine the influence of tip-sample contact area on the electric current output, a control C-AFM test was performed on the HOPG substrate. HOPG offers smooth and renewable surfaces, which are excellent for AFM tests.

a

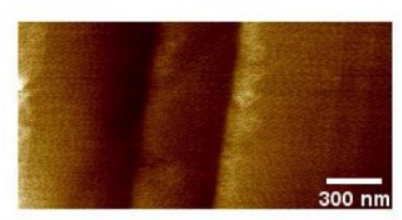

C

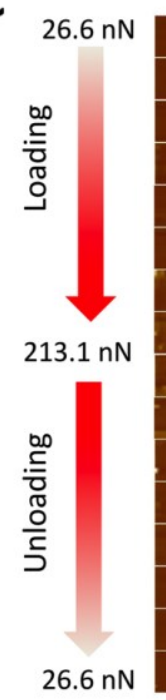

b

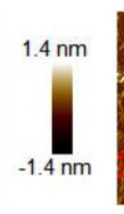

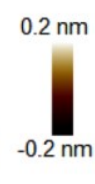

$300 \mathrm{~nm}$

$-0.2 \mathrm{~nm}$

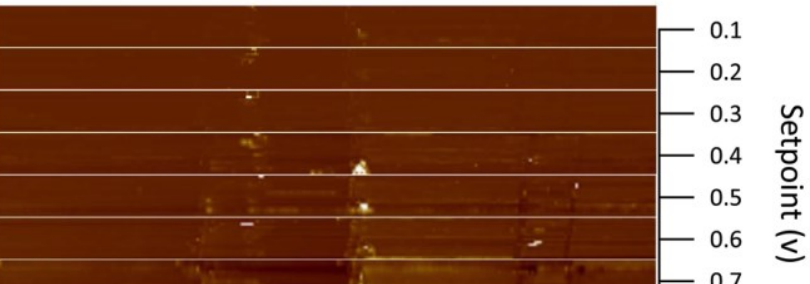

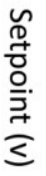

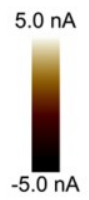

Figure 3-4 (a) Height and (b) deflection maps of the HOPG substrate under a bias voltage of $2 \mathrm{~V}$, (c) Electric current mapping under different localized forces during loading and unloading processes inside the red dashed box in (b). The scale bars are $300 \mathrm{~nm}$. 
Unlike the ionic conductor $\mathrm{LiCoO}_{2}$, natural layered $\mathrm{HOPG}$ is an electron conductor, with an in-plane resistance of $55 \sim 65 \mu \Omega \cdot \mathrm{cm}$ and $6 \sim 7 \mathrm{~m} \Omega \cdot \mathrm{cm}$ resistance along the $c$-axis. ${ }^{28}$ The HOPG substrate has higher resistance along the $c$-axis than the in-plane resistance. Therefore, a higher current output can be expected along the boundaries. During both the loading and unloading processes, the C-AFM images (Figure 3-4c) showed a slight increase in the electric current output when the applied force was increased. Increase in the current output was not only observed along the step edges of the graphite but also appeared to be increased everywhere (Figure S3-9). The same roughness analysis was conducted for the electric current signals (Figure 3-5). When the applied force was increased from 26.6 \pm 1.1 to $213.1 \pm 8.7 \mathrm{nN}$, the $\mathrm{R}_{\mathrm{a}}$ value of the electric current increased from 0.097 to 0.79 $n A$. The corresponding $R_{q}$ value also gave a similar trend (Figure $S 3-10$ ).

The current response observed from the HOPG sample was lower than that from the $\mathrm{LiCoO}_{2}$ sample. This is because the measured resistance between the HOPG substrate and the AFM sample stage was $1.8 \mathrm{M} \Omega$, which is higher than that of the $\mathrm{LiCoO}_{2}$ sample ( $0.8 \mathrm{M} \Omega$ before its resistive switching). The observed high resistance across the HOPG substrate is due to the larger sample thickness $(2 \mathrm{~mm}$ vs. $0.1 \mathrm{~mm})$ and the introduce of substrates. To keep the current output within the detection limit of the TUNA module and to ensure the effect of tip-sample contact areas can be revealed, this high resistance state was kept during the C-AFM tests on the HOPG substrate. Because the HOPG substrate was in direct contact with the AFM tip and there was no ionic contribution within the electric circuit, the effect of tip-sample contact area can be evaluated. 
The HOPG sample is a relatively soft substrate compared with the AFM probe. The Young's modulus and Poisson's ratio of the silicon tip are estimated to be $169 \mathrm{GPa}^{29}$ and 0.22. ${ }^{30}$ However, the Young's modulus and Poisson's ratio of the HOPG substrate are 30 $\mathrm{GPa}^{31-33}$ and $0.24,{ }^{33}$ respectively. Assuming no extra surface forces and low stiffness, the Sneddon analysis model ${ }^{26}$ can be adopted to define the relationship between the tip-sample contact radius $(\boldsymbol{a})$ and the applied force $(\boldsymbol{F})$ :

$$
F=\frac{3}{8} K\left[\left(a^{2}+R^{2}\right) \ln \left(\frac{R+a}{R-a}\right)-2 a R\right]
$$

In Equation (1), $\boldsymbol{F}$ is the applied force, $\boldsymbol{R}$ is the tip radius $(25 \mathrm{~nm})$, and $\boldsymbol{K}$ is the reduced Young's modulus given by:

$$
\frac{1}{K}=\frac{3}{4}\left(\frac{1-v^{2}}{E}+\frac{1-v_{i}^{2}}{E_{i}}\right)
$$

where $\boldsymbol{E}, \boldsymbol{E}_{\boldsymbol{i}}, \boldsymbol{v}$ and $\boldsymbol{v}_{\boldsymbol{i}}$ are the Young's modulus and Poisson's ratios of the silicon tip and the HOPG substrate. The reduced Young's modulus of HOPG was calculated to be 35.43 GPa.

Assuming that there is no existence of insulating films between the tip and the substrate and that the electric contact is in a circular area with a radius $\boldsymbol{a}$, according to Maxwell's theory, ${ }^{27}$ the constriction resistance $\boldsymbol{C}_{\boldsymbol{r}}$ can be given by

$$
C_{r}=\left(\rho_{1}+\rho_{2}\right) / 2 a
$$

where $\rho_{1}$ and $\rho_{2}$ are the specific resistivity of the two materials in contact. The resistivity of the $\mathrm{Pt} / \mathrm{Cr}$ coated silicon probe is estimated to be $3.0 \times 10^{-7} \Omega \cdot \mathrm{m},{ }^{34}$ and the resistivity of HOPG along the $c$-axis is $3.8 \times 10^{-3} \Omega \cdot \mathrm{m} .{ }^{35}$ If the current output $\boldsymbol{I}$ follows Ohm's law under 
the bias voltage $\boldsymbol{U}=2 \mathrm{~V}$, the relationship between the current output $\boldsymbol{I}$ and the applied forces $\boldsymbol{F}$ can be defined as:

$$
F=\frac{3}{8} K\left[\left(\frac{I^{2}\left(\rho_{1}+\rho_{2}\right)^{2}}{4 U^{2}}+R^{2}\right) \ln \left(\frac{2 U R+I \rho_{1}+I \rho_{2}}{2 U R-I \rho_{1}-I \rho_{2}}\right)-\frac{I R\left(\rho_{1}+\rho_{2}\right)}{U}\right]
$$

The experimental value was fitted using Equation 3-4, while the reduced Young's modulus $\boldsymbol{K}$ was kept as the unknown parameter for a nonlinear curve fitting. The two fitted curves gave an estimate $\boldsymbol{K}$ value of $35.6 \mathrm{GPa}$, which is comparable to the theoretical value of 35.43 GPa. The coefficients of determination for the two fitting curves are 0.9009 and 0.9226 , indicating the Sneddon model is suitable for our analysis.

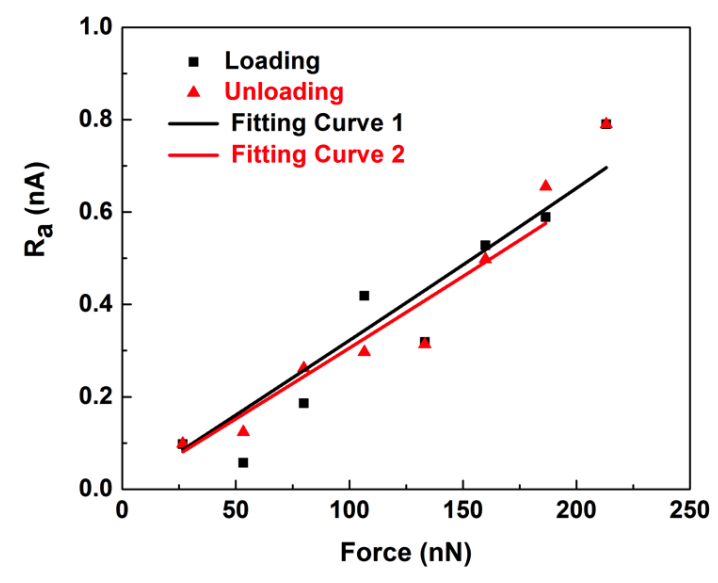

Figure 3-5 Arithmetic mean roughness $\left(\mathrm{R}_{\mathrm{a}}\right)$ of the electric current as a function of applied forces on the HOPG substrate. Fitting curve 1 corresponds to loading, and fitting curve 2 corresponds to unloading.

The Young's modulus of layered $\mathrm{LiCoO}_{2}$ is $174 \mathrm{GPa} \pm 25 \mathrm{GPa}$, measured from individual grains. ${ }^{36}$ Compared with the HOPG substrate, the layered $\mathrm{LiCoO}_{2}$ cathode has 
a higher hardness. Thus, the corresponding reduced Young's modulus should be higher according to Equation 3-2. Since the same kind of probe was used for both substrates, under similar loading condition, the tip-sample contact area on the $\mathrm{LiCoO}_{2}$ substrate would be smaller than that on the HOPG substrate, according to Equation 3-1. Thus, the effect of tipsample contact area on the current output would also be smaller on the $\mathrm{LiCoO}_{2}$ substrate. On the other hand, the current increase on the HOPG substrate (1.15 nA increase from $0.268 \mathrm{nA}$ to $1.42 \mathrm{nA})$ is relatively limited compared with that on the $\mathrm{LiCoO}_{2}(139.4 \mathrm{nA}$ increase from 43.6 to $183.0 \mathrm{nA}$ ) under the same electric field. Therefore, the effect of tipsample contact area on the electric current output would be limited and ignorable for the $\mathrm{LiCoO}_{2}$ substrate. The increase in current response on the $\mathrm{LiCoO}_{2}$ substrate should be mainly due to the redistribution of lithium ions.
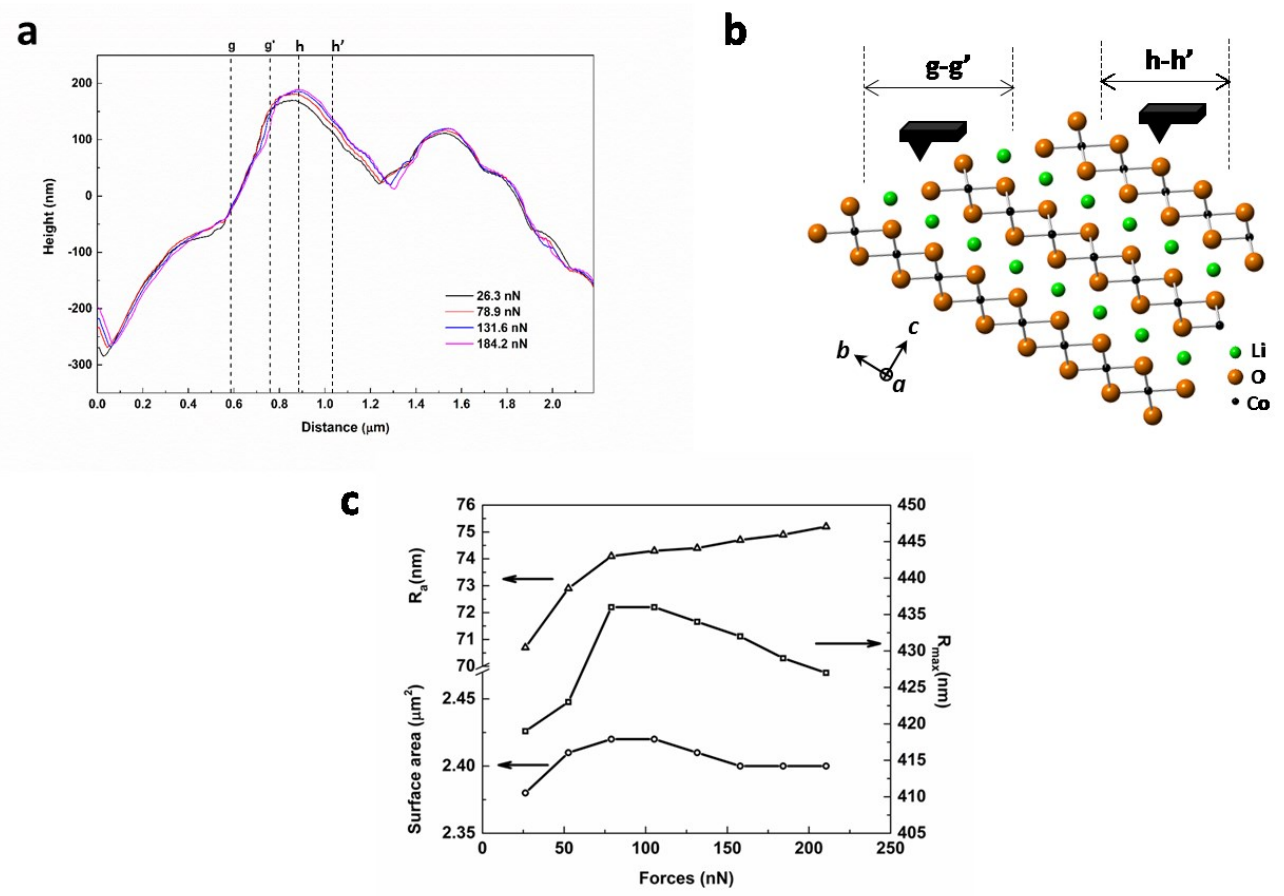
Figure 3-6 (a) Cross-section analysis of the height images of the layered $\mathrm{LiCoO}_{2}$ (Figure S11a) along the same diagonal line e-e' as Figure $2 \mathrm{~b}$ during the loading process. (b) An atomic model of the layered $\mathrm{LiCoO}_{2}$ with a proposed orientation at the g-g' and h-h' locations. (c) Variation of height roughness $\left(R_{a}\right.$ and $\left.R_{\max }\right)$ and the surface area of the layered $\mathrm{LiCoO}_{2}$ as a function of applied forces.

To develop a better understanding of the stress-induced redistribution of lithium ions, detailed analysis of the morphology change on the $\mathrm{LiCoO}_{2}$ substrate was also conducted. The topography changes as a function of applied stress were shown in supporting information Figure S3-11. The obtained topography images were stable, indicating no sample damaged was involved. Compared with the cross-section analysis of the current signals (Figure 3-2b), the conduction region g-g' showed no obvious surface expansion, which may indicate this grain is oriented with its (001) plane close to the direction of electric field, proposed as Figure 3-6b. Therefore, minimum expansion or even reduced surface height was observed (Figure 3-6a). For the region h-h', it belongs to the same layered $\mathrm{LiCoO}_{2}$ grain with the region g-g'. Although no current output was observed, obvious volume expansion was detected. Since these two regions were in the same grain, it is highly possible that the volume expansion detected along the h-h' region was due to the redistribution of lithium ions inside the g-g' region, with a grain orientation proposed as Figure 3-2b. With the increase of applied forces, the volume expansion at the $h-h$ ' region increased, which should be related to the enhanced redistribution of lithium ions at the gg' region. 
The resistive switching of the layered $\mathrm{LixCoO}_{2}$ happens with a lithium concentration within the range of $0.75 \leq x \leq 0.94$, where the diffusion of lithium ions is preferred through the intermediate oxygen-tetrahedral sites $\left(\boldsymbol{P}_{2}\right)$ other than a direct jump between two lithium octahedral sites $\left(\boldsymbol{P}_{I}\right)$ (Figure S3-12). ${ }^{37}$ The transformation mechanism from $\mathrm{LiCoO}_{2}$ to $\mathrm{Li}_{0.75} \mathrm{CoO}_{2}$ was proposed to be an ordering of lithium atoms and vacancies within the (001) lithium layers. ${ }^{38}$ The overall dimension of the layered $\mathrm{LiCoO}_{2}$ crystals does not change based on in situ XRD analysis ${ }^{15}$ and the theoretical modeling ${ }^{38}$. Previous modeling work proved stress-enhanced diffusion of lithium ions, rather than diffusioninduced stress, will dominate at a high lithium concentration in amorphous lithium alloy nanowires. ${ }^{39}$ In our case, the applied external stress may facilitate the diffusion of lithium ions or vacancies and further result in resistive switching of the layered $\mathrm{LiCoO}_{2}$. The diffusion of vacancies driven by the stress gradient has also been observed in other semiconductors ${ }^{40}$ and alloys ${ }^{41}$.

For the further transformation from $\mathrm{Li}_{0.75} \mathrm{CoO}_{2}$ to $\mathrm{Li}_{0.5} \mathrm{CoO}_{2}$, only one hexagonal phase exists and the substrate will be in a conductive state, similar to the condition observed along the region g-g'. Along with the further delithiation, a lattice expansion along $c$-axis has been observed ${ }^{15}$, which is consistent with that observed at the h-h' region. When lithium ions are driven away from their original location, leaving two negatively charged $\mathrm{CoO}_{2}$ layers facing each other. The electrostatic repulsions between the two negatively charged $\mathrm{CoO}_{2}$ layers will induce lattice expansion along the $c$-axis of the layered $\mathrm{LiCoO}_{2}{ }^{15}$ Therefore, increased roughness $\left(\mathrm{R}_{\mathrm{a}}\right)$ and surface area were observed (Figure 3-6c). 

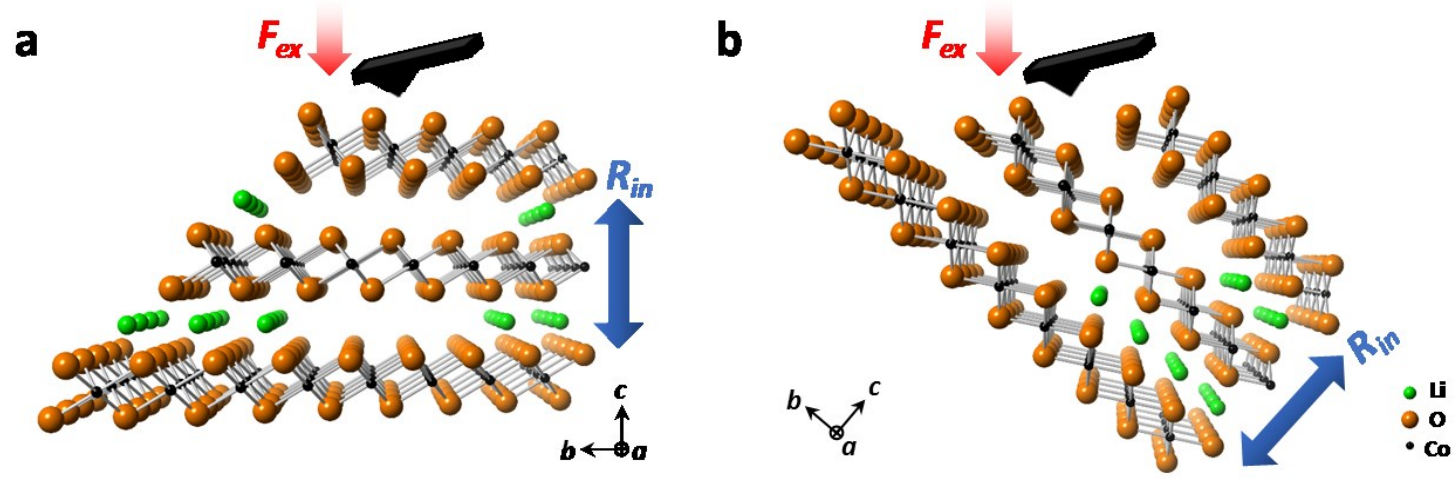

Figure 3-7 The atomic model of the diffusion of lithium ions inside the layered $\mathrm{LiCoO}_{2}$ cathode with its (001) plane oriented perpendicular to the electric field (a) or close to the electric field (b) under an applied force $\boldsymbol{F}_{\boldsymbol{e x}}$. The repulsive forces between the $\mathrm{CoO}_{2}$ layers were indicated as $\boldsymbol{R}_{\boldsymbol{i n}}$.

If the $\mathrm{LiCoO}_{2}$ grain was oriented with its (001) plane perpendicular to the electric field (Figure 3-7a), it is possible that the expansion of $\mathrm{CoO}_{2}$ layers was suppressed by the external stress field. The electrostatic force between two adjacent $\mathrm{CoO}_{2}$ layers is close to $4.19 \mathrm{nN}$, given the $4.68 \AA$ distance between two adjacent $\mathrm{CoO}_{2}$ layers. ${ }^{42}$ This electrostatic force is relatively small compared with the applied forces. Taking multiple layers of $\mathrm{CoO}_{2}$ into consideration, when the applied force $\left(\boldsymbol{F}_{\boldsymbol{e x}}\right)$ is large enough to overcome this repulsive force $\left(\boldsymbol{R}_{\boldsymbol{i n}}\right)$, the expansion of the $\mathrm{CoO}_{2}$ layers could be suppressed. The actual surface expansion will show the compensation between these two forces. This is consistent with the reduced volume expansion with the linear increase of applied forces, observed along the region h-h' (Figure 3-6a). However, if the $\mathrm{LiCoO}_{2}$ grain was oriented with its (001) plane close to the electric field (Figure 3-7b), repulsive forces generated from the deficiency of lithium ions cannot be fully compensated by the external stresses. This would 
also result in a reduced surface height, which is similar to that observed at g-g' region (Figure 3-6a). Along with the reduced surface height, a deeper penetration depth of the electric field can be expected, and more lithium ions will be driven away from the layered $\mathrm{LiCoO}_{2}$. This is consistent with the observation that the surface area, $\mathrm{R}_{\max }$, and increasing rate of $R_{a}$ were all reduced when a higher force was applied (Figure 3-6c).

\subsection{Conclusions}

The study of lithium ion transportation in electrode materials and the influencing factors for this property are critical for the design of high-rate and high-performance lithium ion batteries. Our results reveal that localized mechanical stresses can facilitate the redistribution of lithium ions inside the layered $\mathrm{LiCoO}_{2}$. Conductive AFM tests, combined with the dynamic force ramping technique, further demonstrated the applied stress was capable of inducing reversible resistive switching of the layered $\mathrm{LiCoO}_{2}$ cathode. The approach presented here offers guidance for future studies of ionic redistribution in other layered materials. With the suppressed expansion of the $\mathrm{CoO}_{2}$ layers, a deeper penetration depth of the electric field was proposed to be responsible for the enhanced redistribution of lithium ions and the increased current responses from the $\mathrm{LiCoO}_{2}$ substrate. Currently, the mechanical stress applied is localized and the forces are limited in nanoscale $(20 \sim 200 \mathrm{nN})$. Further study of this influencing factor at the higher level or introducing mechanical loading to battery cells during electrochemical cycling would also be beneficial for practical application. Also, if the temperature effect on the redistribution of lithium ions under different stress field can be studied, the thermodynamic driving force of the diffusion of lithium ions can also be estimated quantitatively. 


\subsection{References}

1 Tarascon, J.-M. \& Armand, M. Issues and challenges facing rechargeable lithium batteries. Nature 414, 359-367 (2001).

2 Lu, L., Han, X., Li, J., Hua, J. \& Ouyang, M. A review on the key issues for lithium-ion battery management in electric vehicles. Journal of Power Sources 226, 272-288 (2013).

3 Smith, K. \& Wang, C.-Y. Power and thermal characterization of a lithium-ion battery pack for hybrid-electric vehicles. Journal of Power Sources 160, 662-673 (2006).

4 Hosono, E., Kudo, T., Honma, I., Matsuda, H. \& Zhou, H. Synthesis of single crystalline spinel $\mathrm{LiMn}_{2} \mathrm{O}_{4}$ nanowires for a lithium ion battery with high power density. Nano Letters 9, 1045-1051 (2009).

5 Bruce, P. G., Scrosati, B. \& Tarascon, J. M. Nanomaterials for rechargeable lithium batteries. Angewandte Chemie International Edition 47, 2930-2946 (2008).

6 Nie, A. et al. Atomic-scale observation of lithiation reaction front in nanoscale $\mathrm{SnO}_{2}$ materials. ACS Nano 7, 6203-6211 (2013).

$7 \quad$ Yuan, Y. et al. Asynchronous Crystal Cell Expansion during Lithiation of $\mathrm{K}^{+}-$ Stabilized $\alpha-\mathrm{MnO}_{2}$. Nano Letters 15, 2998-3007 (2015).

8 Balke, N. et al. Nanoscale mapping of ion diffusion in a lithium-ion battery cathode. Nature Nanotechnology 5, 749 (2010).

9 Yuan, Y. et al. Atomistic insights into the oriented attachment of tunnel-based oxide nanostructures. ACS Nano (2015).

10 Kannan, A., Rabenberg, L. \& Manthiram, A. High capacity surface-modified $\mathrm{LiCoO}_{2}$ cathodes for lithium-ion batteries. Electrochemical and Solid-State Letters 6, A16-A18 (2003).

11 Kim, Y. J. et al. Electrochemical stability of thin-film $\mathrm{LiCoO}_{2}$ cathodes by aluminum-oxide coating. Chemistry of Materials 15, 1505-1511 (2003).

12 Motohashi, T. et al. Impact of lithium composition on the thermoelectric properties of the layered cobalt oxide system $\mathrm{Li}_{\mathrm{x}} \mathrm{CoO}_{2}$. Physical Review B 83, 195128 (2011). 
13 Xia, H., Lu, L., Meng, Y. \& Ceder, G. Phase transitions and high-voltage electrochemical behavior of $\mathrm{LiCoO}_{2}$ thin films grown by pulsed laser deposition. Journal of the Electrochemical Society 154, A337-A342 (2007).

14 Nie, A. et al. Twin Boundary-Assisted Lithium Ion Transport. Nano Letters 15, 610-615 (2014).

15 Amatucci, G., Tarascon, J. \& Klein, L. $\mathrm{CoO}_{2}$, the end member of the $\mathrm{LiCoO}_{2}$ solid solution. Journal of The Electrochemical Society 143, 1114-1123 (1996).

16 Sun, H. et al. Direct observation of conversion between threshold switching and memory switching induced by conductive filament morphology. Advanced Functional Materials 24, 5679-5686 (2014).

17 Sun, J. et al. In situ observation of nickel as an oxidizable electrode material for the solid-electrolyte-based resistive random access memory. Applied Physics Letters 102, 053502 (2013).

18 Xu, Z., Bando, Y., Wang, W., Bai, X. \& Golberg, D. Real-time in situ HRTEMresolved resistance switching of $\mathrm{Ag}_{2} \mathrm{~S}$ nanoscale ionic conductor. ACS Nano 4, 2515-2522 (2010).

19 Chen, X. et al. Nanoscale magnetization reversal caused by electric field-induced ion migration and redistribution in cobalt ferrite thin films. ACS Nano 9, 42104218 (2015).

20 Zhu, X. et al. Direct observation of lithium-ion transport under an electrical field in $\mathrm{Li}_{\mathrm{x}} \mathrm{CoO}_{2}$ nanograins. Scientific Reports 3 (2013).

21 Balke, N. et al. Local detection of activation energy for ionic transport in lithium cobalt oxide. Nano Letters 12, 3399-3403 (2012).

22 Balke, N. et al. Three-dimensional vector electrochemical strain microscopy. Journal of Applied Physics 112, 052020 (2012).

23 Sader, J. E., Chon, J. W. \& Mulvaney, P. Calibration of rectangular atomic force microscope cantilevers. Review of Scientific Instruments 70, 3967-3969 (1999).

24 Mohideen, U. \& Roy, A. Precision measurement of the Casimir force from 0.1 to $0.9 \mu \mathrm{m}$. Physical Review Letters 81, 4549 (1998).

25 Moradpour, A. et al. Resistive switching phenomena in $\mathrm{Li}_{\mathrm{x}} \mathrm{CoO}_{2}$ thin films. Advanced Materials 23, 4141-4145 (2011).

26 Brunero Cappella, G. D. Force-distance curves by atomic force microscopy. Surface Science Reports 34, 1-104 (1999). 
27 Sawada, S., Tsukiji, S., Shimada, S., Tamai, T. \& Hattori, Y. in Electrical Contacts (Holm), 2012 IEEE 58th Holm Conference on. 1-6 (IEEE).

28 Edman, L., Sundqvist, B., McRae, E. \& Litvin-Staszewska, E. Electrical resistivity of single-crystal graphite under pressure: An anisotropic threedimensional semimetal. Physical Review B 57, 6227 (1998).

29 Vinckier, A. \& Semenza, G. Measuring elasticity of biological materials by atomic force microscopy. Febs Letters 430, 12-16 (1998).

30 Volodin, A. et al. Imaging the elastic properties of coiled carbon nanotubes with atomic force microscopy. Physical Review Letters 84, 3342 (2000).

31 Eskelsen, J. R. et al. Correlating elastic properties and molecular organization of an ionic organic nanostructure. Nanoscale 6, 316-327 (2014).

32 Trtik, P., Kaufmann, J. \& Volz, U. On the use of peak-force tapping atomic force microscopy for quantification of the local elastic modulus in hardened cement paste. Cement and Concrete Research 42, 215-221 (2012).

33 Tsuji, T. \& Yamanaka, K. Observation by ultrasonic atomic force microscopy of reversible displacement of subsurface dislocations in highly oriented pyrolytic graphite. Nanotechnology 12, 301 (2001).

34 Bhushan, B., Kwak, K. J. \& Palacio, M. Nanotribology and nanomechanics of AFM probe-based data recording technology. Journal of Physics: Condensed Matter 20, 365207 (2008).

35 Koren, E., Knoll, A., Lörtscher, E. \& Duerig, U. Direct experimental observation of stacking fault scattering in highly oriented pyrolytic graphite meso-structures. Nature Communications 5 (2014).

36 Qu, M. et al. Nanomechanical quantification of elastic, plastic, and fracture properties of $\mathrm{LiCoO}_{2}$. Advanced Energy Materials 2, 940-944 (2012).

37 Van der Ven, A. \& Ceder, G. Lithium diffusion in layered $\mathrm{Li}_{\mathrm{x}} \mathrm{CoO}_{2}$. Electrochemical and Solid-State Letters 3, 301-304 (2000).

38 Ben Yahia, H., Shikano, M. \& Kobayashi, H. Phase Transition mechanisms in $\mathrm{Li}_{\mathrm{x}} \mathrm{CoO}_{2}(0.25 \leq \mathrm{x} \leq 1)$ based on group-subgroup transformations. Chemistry of Materials 25, 3687-3701 (2013).

39 Gao, Y. \& Zhou, M. Strong stress-enhanced diffusion in amorphous lithium alloy nanowire electrodes. Journal of Applied Physics 109, 014310 (2011). 
40 Daw, M. S., Windl, W., Carlson, N. N., Laudon, M. \& Masquelier, M. P. Effect of stress on dopant and defect diffusion in Si: A general treatment. Physical Review B 64, 045205 (2001).

41 Shao, W., Gan, Z., Mhaisalkar, S., Chen, Z. \& Li, H. The effect of line width on stress-induced voiding in $\mathrm{Cu}$ dual damascene interconnects. Thin Solid Films 504, 298-301 (2006).

42 Shao-Horn, Y., Croguennec, L., Delmas, C., Nelson, E. C. \& O'Keefe, M. A. Atomic resolution of lithium ions in $\mathrm{LiCoO}_{2}$. Nature Materials 2, 464-467 (2003). 


\section{Chapter 4 Energy-driven surface evolution in beta- $\mathrm{MnO}_{2}$ structures $^{2}$}

Exposed crystal facets directly affect the electrochemical/catalytic performance of $\mathrm{MnO}_{2}$ materials during their applications in supercapacitors, rechargeable batteries, and fuel cells. Currently, the facet-controlled synthesis of $\mathrm{MnO}_{2}$ is facing serious challenges due to the lack of an in-depth understanding of their surface evolution mechanisms. Here, combining aberration-corrected scanning transmission electron microscopy (STEM) and high-resolution TEM, we revealed a mutual energy-driven mechanism between beta$\mathrm{MnO}_{2}$ nanowires and microstructures that dominated the evolution of the lateral facets in both structures. The evolution of the lateral surfaces followed the elimination of the $\{100\}$ facets and increased the occupancy of $\{110\}$ facets with the increase in hydrothermal retention time. Both self-growth and oriented attachment along their $\{100\}$ facets were observed as two different ways to reduce the surface energies of the beta- $\mathrm{MnO}_{2}$ structures. High-density screw dislocations with the $1 / 2<100>$ Burgers vector were generated consequently. The observed surface evolution phenomenon offers guidance for the facetcontrolled growth of beta- $\mathrm{MnO}_{2}$ materials with high performances for its application in metal-air batteries, fuel cells, supercapacitors, etc.

2 The material contained in this chapter was previously published in Nano Research. (Reprinted with permission from Yao, W., Yuan, Y., Asayesh-Ardakani, H., Huang Z., Long F., Friedrich C., Amine K., Lu, J., \& Shahbazian-Yassar, R. Nano Research, 2018, 11(1), 206-215. Copyright 2018, Springer Nature.) 


\subsection{Introduction}

Beta phase manganese dioxide $\left(\beta-\mathrm{MnO}_{2}\right)$ has been widely used as an efficient material for supercapacitors ${ }^{1}$, lithium ion batteries ${ }^{2,3}$, catalysts for lithium-air batteries, and water treatement ${ }^{4-6}$. Silver modified $\beta-\mathrm{MnO}_{2}$ nanorods were used an effective cathode catalyst for oxygen reduction reactions in lithium-air batteries ${ }^{7}$. High surface areas enabled $\beta$ $\mathrm{MnO}_{2}$ nanowires to be used as a robust catalyst for water treatment ${ }^{8}$. To improve the performance of the $\beta-\mathrm{MnO}_{2}$ materials, critical size control and morphological modification are commonly needed. As the electrode material for supercapacitors, $\beta-\mathrm{MnO}_{2}$ nanorods (50 100 $\mathrm{nm}$ in width, $300 \mathrm{~nm}$ in length) showed a capacitance around $28 \mathrm{~F} \cdot \mathrm{g}^{-1}$, however, $\beta-\mathrm{MnO}_{2}$ nanowires with much smaller diameters $(<10 \mathrm{~nm})$, gave a reported capacitance of $294 \mathrm{~F} \cdot \mathrm{g}^{-1}$ at $1 \mathrm{~A} \mathrm{~g}^{-1}$, which is almost ten times higher and also comparable with that of the alpha phase $\mathrm{MnO}_{2}{ }^{10}$. As the positive electrode for lithium ion batteries, bulk $\beta-\mathrm{MnO}_{2}(<$ $20 \mathrm{mAh} \mathrm{g}^{-1}$ ) is usually inactive for lithium intercalation due to thermal decomposition or poor kinetics ${ }^{11,12}$, however, mesoporous $\beta-\mathrm{MnO}_{2}$ delivered a reversible capacity $\sim 284$ $\mathrm{mAh} \mathrm{g}^{-1}$ at a rate of $15 \mathrm{~mA} \mathrm{~g}^{-13}$, and hollow $\beta-\mathrm{MnO}_{2}$ bipyramid maintained a $213 \mathrm{mAh} \mathrm{g}$ ${ }^{1}$ capacity at a rate of $100 \mathrm{~mA} \mathrm{~g}^{-1}$ for 150 cycles $^{3}$. Thus, morphology control of the high crystalline $\beta-\mathrm{MnO}_{2}$ material is compulsory to improve its electrochemical performance.

Except for size ${ }^{1}$ and morphology ${ }^{3,11,14,15}$, the exposed crystal surfaces directly affect the performance of the $\beta-\mathrm{MnO}_{2}$ material. Recently, several modeling works studied the properties of different $\beta-\mathrm{MnO}_{2}$ crystal surfaces ${ }^{16-19}$. Oxford, et al simulated the reconstruction of the $\beta-\mathrm{MnO}_{2}$ (110), (100), and (101) surfaces under oxidation or reduction based on ab initio thermodynamics ${ }^{16}$. Tompsett, et al calculated the surface energies of 
different $\beta-\mathrm{MnO}_{2}$ surfaces using density functional theory (DFT) ${ }^{19}$. The lithium ion migration at different surfaces of the $\beta-\mathrm{MnO}_{2}$ crystals was also simulated, and a higher lithium ion migration barrier at the (101) surfaces was predicted than that at the (001) surfaces and the bulk ${ }^{17}$. Unfortunately, no experimental result has been reported to support these computational results so far. A detailed analysis of the exposed crystal surfaces in $\beta$ $\mathrm{MnO}_{2}$ is highly needed to develop the understanding of the correlation between its facets and the corresponding electrochemical/catalytic performance. The facet-dependent electrochemical performance was also observed in other materials, such as $\mathrm{Pt}^{20,21}, \mathrm{Pd}^{22}$, $\mathrm{Au}^{23}, \mathrm{TiO}_{2}{ }^{24}, \mathrm{Co}_{3} \mathrm{O}_{4}{ }^{25}$, etc.

Low-temperature hydrothermal route was commonly used for the synthesis of high purity, well-distributed $\beta-\mathrm{MnO}_{2}$ material with different morphologies ${ }^{26-30}$. Previous studies of the growth mechanism of the $\beta-\mathrm{MnO}_{2}$ materials mainly focused on the phase transformation process at the early stage of synthesis ${ }^{31,32}$. Little attention was paid to the evolution of the exposed facets during the long-time synthesis process. Here, combing aberration-corrected STEM and high-resolution TEM, we revealed a coherent energycontrolled surface evolution mechanism for both $\beta-\mathrm{MnO}_{2}$ nanowires and microrods. Due to the higher surface energy of the $\{100\}$ facets, under longer hydrothermal reaction time, a reduced occupancy of $\{100\}$ facets but higher occupancy of $\{110\}$ facets was observed among the lateral surfaces of both structures. Our findings offer guidance for facetcontrolled growth of $\beta-\mathrm{MnO}_{2}$ materials for its wide applications in the field of energy storage as well as catalysts. 


\subsection{Experimental}

\subsubsection{Materials synthesis}

$\beta-\mathrm{MnO}_{2}$ nanowires and microrods were synthesized following a typical hydrothermal growth method reported elsewhere ${ }^{33}$. For the $\beta-\mathrm{MnO}_{2}$ nanowires, $2.0 \mathrm{mmol}\left(\mathrm{NH}_{4}\right)_{2} \mathrm{~S}_{2} \mathrm{O}_{8}$ and $2.0 \mathrm{mmol} \mathrm{MnSO}_{4} \cdot \mathrm{H}_{2} \mathrm{O}$ were mixed thoroughly in $20 \mathrm{ml}$ deionized (DI) water (18.2 $\left.\mathrm{M} \Omega \cdot \mathrm{cm}^{-1}\right)$. To regulate the growth of the $\beta-\mathrm{MnO}_{2}$ nanowires, $2.0 \mathrm{~g}$ of cetyltrimethylammonium bromide (CTAB) was added as the cationic surfactant. The mixture was stirred continuously for $30 \mathrm{~min}$, then transferred to a $40 \mathrm{~mL}$ Teflon-lined stainless-steel autoclave, sealed, and heated at $140{ }^{\circ} \mathrm{C}$ for $12 \mathrm{~h}, 18 \mathrm{~h}$, and $24 \mathrm{~h}$, respectively. For the synthesis of $\beta-\mathrm{MnO}_{2}$ microrods, $1.6 \mathrm{mmol} \mathrm{KMnO}_{4}, 2.4 \mathrm{mmol} \mathrm{MnCl}_{2} \cdot 4 \mathrm{H}_{2} \mathrm{O}$, and $4.0 \mathrm{mmol} \mathrm{KCl}$ were added into $20 \mathrm{~mL}$ DI water under constant stirring. The mixture was stirred for 30 min until a well-distributed purple solution was formed. The obtained solution was then transferred to a $40 \mathrm{~mL}$ Teflon-lined stainless-steel autoclave, sealed, and heated at $180{ }^{\circ} \mathrm{C}$ for $12 \mathrm{~h}, 24 \mathrm{~h}$, and $48 \mathrm{~h}$, respectively. Both the nanowires and microrods were collected by centrifugation, followed by consecutively cleaning with DI water and ethyl alcohol, and drying at $60{ }^{\circ} \mathrm{C}$ in air for $12 \mathrm{~h}$.

\subsubsection{Cross section sample preparation}

To prepare the cross-section samples, the nanowires or microrods were first mixed with cold mounting epoxy resin (EPOFIX, Electron Microscopy Sciences) and hardener (EPOFIX, Triethylenetetramine, Electron Microscopy Sciences). The mixture was sonicated for 10 min to reduce bubble formation. After the mixture was fully solidified, it 
was mounted on a Leica Ultracut UCT ultramicrotome and sliced with a diamond knife. The feeding step size was $500 \mathrm{~nm}$.

\subsubsection{Materials characterization}

The phases of the synthesized $\mathrm{MnO}_{2}$ were analyzed using a Scintag XDS2000 powder Xray diffractometer. X-ray diffraction (XRD) peaks were collected with $\mathrm{Cu} \mathrm{K} \alpha$ radiation. The scan step was $0.02^{\circ} \mathrm{min}^{-1}$. Hitachi S-4700 field emission scanning electron microscope (FE-SEM) was used to check the morphologies. The growth direction and inner structure

of the $\mathrm{MnO}_{2}$ nanowires and microrods were further identified by high-resolution transmission electron microscopy (HRTEM, JEOL 3010) under an accelerating voltage of $300 \mathrm{kV}$. Atomic scale study of the $\beta-\mathrm{MnO}_{2}$ cross-section structures was conducted on a spherical aberration-corrected JEOL JEM-ARM200CF STEM. The microscope is equipped with a 200-kV cold-field emission gun, annular bright field (ABF) and high angle annular dark field (HAADF) detectors. A 22-mrad-probe convergence angle was used for acquiring all STEM images. The HAADF images were captured using a 90-mrad innerdetector angle.

\subsection{Results and discussion}

\subsubsection{Facet evolution of the $\beta-\mathrm{MnO}_{2}$ nanowires}

The synthesis of $\beta-\mathrm{MnO}_{2}$ nanowire used $\mathrm{MnSO}_{4}$ as the main manganese source. The $\beta$ $\mathrm{MnO}_{2}$ nanowires were obtained based on the redox reaction between $\mathrm{Mn}^{2+}$ and $\mathrm{S}_{2} \mathrm{O}_{8}{ }^{2-}$. The thickness of the obtained $\beta-\mathrm{MnO}_{2}$ nanowires was $50 \sim 100 \mathrm{~nm}$, with a length $\sim 1 \mu \mathrm{m}$ (Figure 4-1a). The structure of the nanowires was confirmed to be single-phase tetragonal $\beta-\mathrm{MnO}_{2}$ 
crystal (JCPDS 24-0735) with a lattice parameter of $a=b=4.398 \AA, c=2.869 \AA$ (Figure S4-1). The acquired XRD patterns under different hydrothermal reaction time are all in good agreement with the standard (Figure 4-1b). The HRTEM images (Figure 1c, d), taken from the lateral side of the nanowires, confirmed the growth direction of the $\beta-\mathrm{MnO}_{2}$ nanowires was along its [001] tunnel direction. The high-resolution lattice image (Figure 1d) was obtained at the $[1 \overline{1} 0]$ zone axis.

a

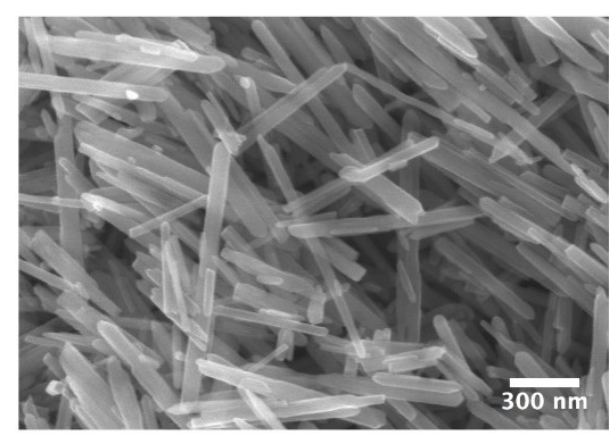

C

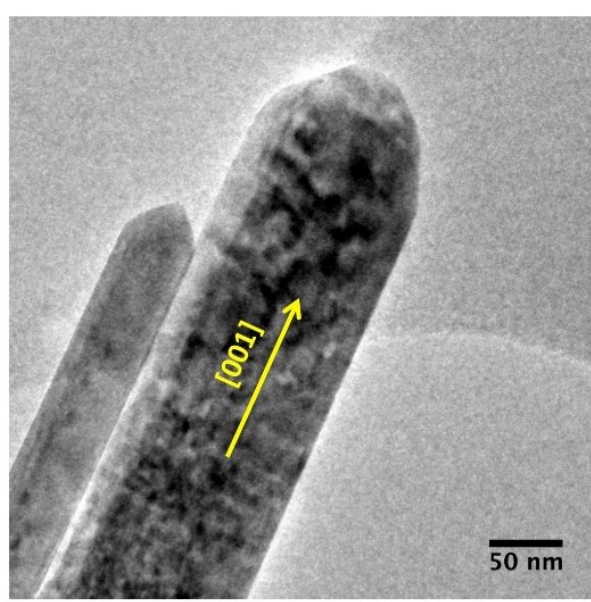

b

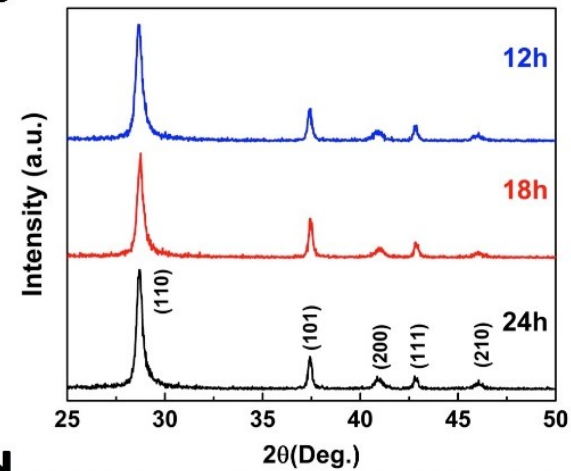

d

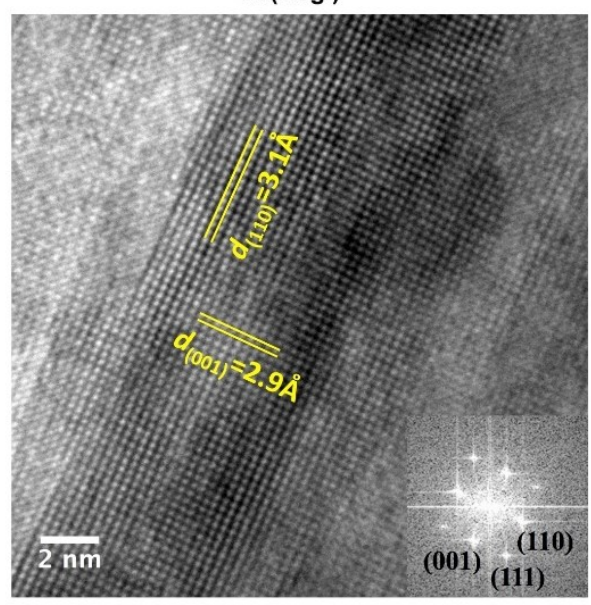

Figure 4-1 Morphology and structure characterization of the synthesized $\beta-\mathrm{MnO}_{2}$ nanowires. (a) SEM image of synthesized $\beta-\mathrm{MnO}_{2}$ nanowires. Hydrothermally grown at $140^{\circ} \mathrm{C}$ for $12 \mathrm{~h}$. (b) The powder XRD pattern of the $\beta-\mathrm{MnO}_{2}$ nanowires obtained under different hydrothermal reaction time. (c) Low magnification TEM image of $\beta-\mathrm{MnO}_{2}$ 
nanowires. The inset panel gives the corresponding Fast Fourier Transform (FFT) image.

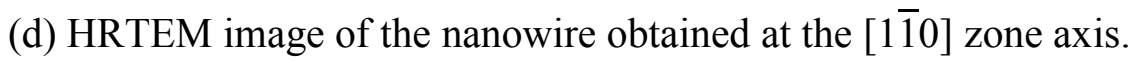

Analysis of the cross-section samples was shown in Figure 4-2. The cross-section of the $\beta-\mathrm{MnO}_{2}$ nanowire was found to be octagonal-shaped (Figure 4-2a). The atomic scale HAADF image (Figure 4-2b) gives clearly the structure of the $1 \times 1$ tunnels inside the $\beta$ $\mathrm{MnO}_{2}$ nanowire. Lateral surfaces of the $\beta-\mathrm{MnO}_{2}$ nanowire were identified to be the $\{110\}$ and $\{100\}$ facets. Figure $4-2 \mathrm{c}$ gives the atomic model of the $\beta-\mathrm{MnO}_{2}$ nanowire viewed along the $c$-axis with the $\{110\}$ and $\{100\}$ planes as the lateral surfaces. The $\{110\}$ and $\{100\}$ facets have different arrangements of manganese and oxygen atoms (Figure S4-2). The manganese atoms on the $\{110\}$ facets are arranged as a flat structure, while the manganese atoms on the $\{100\}$ facets give a serrated structure. The surface energies of $\{110\}$ and $\{100\}$ facets were calculated to be 0.62 and $0.94 \mathrm{~J} \mathrm{~m}^{-2}$ based on a $\mathrm{PBE}+\mathrm{U}$ method ${ }^{19}$. With a lower surface energy, the $\{110\}$ planes were predicted to dominate the surface of $\beta-\mathrm{MnO}_{2}$ crystals.

For the octagonal-shaped cross-sections, the $\{110\}$ facets were connected through the $\{100\}$ facets located at the four corners. Except the octagonal shape, other cross-section structures with fewer $\{100\}$ facets were also observed among the nanowires obtained at 12h (Figure 4-2d). Several line defects were observed inside the cross-section samples (Figure 4-2d). High-resolution HAADF images (Figure S4-3) confirmed that these line defects were exactly along the $\{100\}$ planes. Since the $\{100\}$ facets are less stable that the $\{110\}$ facets, they tend to be eliminated during the growth of the nanowires to reduce the 
overall surface energy. This is in consistent with the observation from XRD patterns. When hydrothermal reaction time increased from $12 \mathrm{~h}$ to $24 \mathrm{~h}$, the integrated peak intensity ratio $\mathrm{I}_{(110)} / \mathrm{I}_{(200)}$ increased from 10.472 to 11.255 , which indicate a preferred occupancy of the relatively stable $\{110\}$ facets. Elimination of the $\{100\}$ facets is due to two reasons. On one hand, the $\{100\}$ facets have higher surface energies; on the other hand, the serrated structure of the $\{100\}$ facets is easier for the attachment of unsaturated $\left[\mathrm{MnO}_{\mathrm{x}}\right]$ radicals to form the $1 \times 1$ tunnel structures.
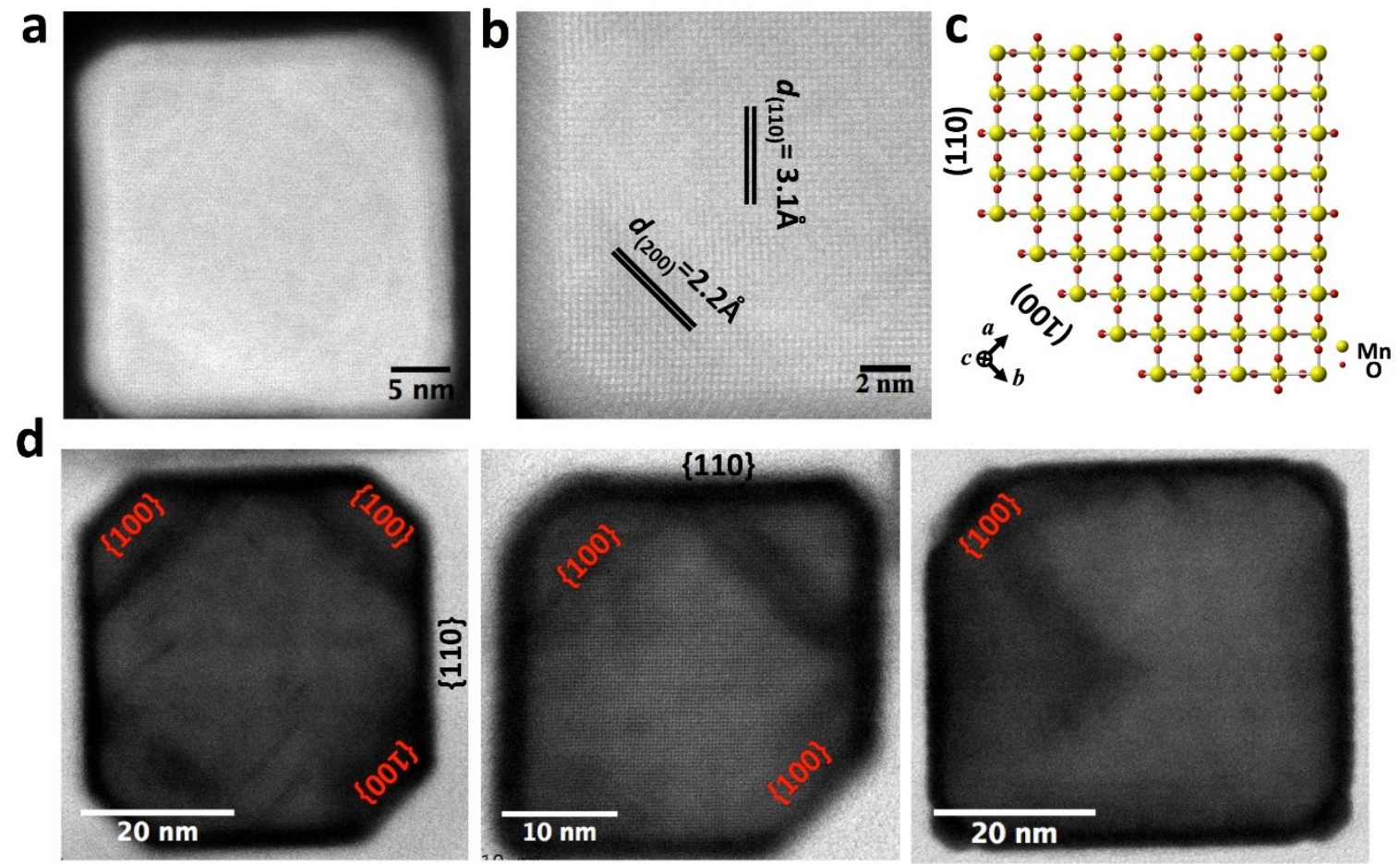

Surface-energy-driven growth of $\beta-\mathrm{MnO}_{2}$ nanowires

Figure 4-2 Atomic-scale STEM imaging of the cross-sections of $\beta-\mathrm{MnO}_{2}$ nanowire obtained at 12h. $(\mathrm{a}, \mathrm{b}) \mathrm{HAADF}$ cross-section images of the $\beta-\mathrm{MnO}_{2}$ nanowire viewed along its [001] direction. (c) Corresponding atomic model of the $\beta-\mathrm{MnO}_{2}$ cross-section 
with (110) and (100) facets. (d) $\mathrm{ABF}$ images of $\beta-\mathrm{MnO}_{2}$ cross-sections with different amount of $\{100\}$ facets. All viewed along the [001] direction.

The elimination of $\{100\}$ facets can also be realized through oriented attachment among different $\beta-\mathrm{MnO}_{2}$ nanowires, as shown in Figure 4-3a. The similar phenomenon was observed previously from $\alpha-\mathrm{MnO}_{2}$ nanowires, where two $\alpha-\mathrm{MnO}_{2}$ nanowires attached to each other along the oriented $\{110\}$ facets to reduce the overall energy ${ }^{34}$. For $\beta-\mathrm{MnO}_{2}$ nanowires, the less stable $\{100\}$ facets become the preferred facets for oriented attachment. Line defects were also generated during the oriented attachment along the attached $\{100\}$ plans (Figure 4-3b). These line defects appeared to be screw dislocations with $1 / 2<100>$ burgers vector. The generated line defects were all along the $\{100\}$ planes during both selfgrowth and oriented attachment process.
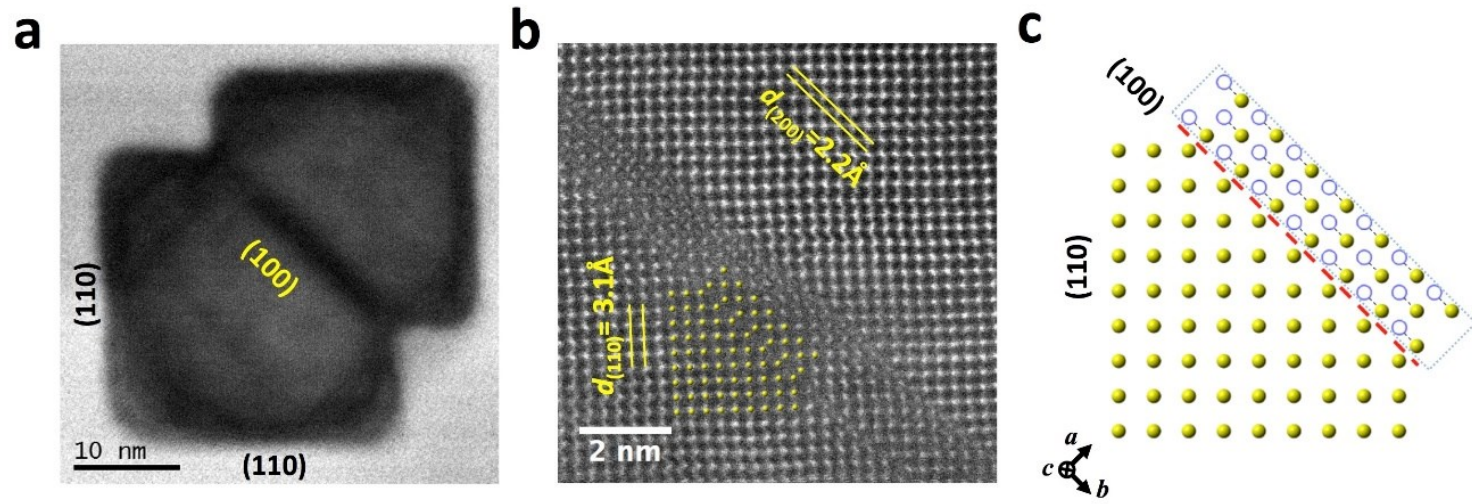

Figure 4-3 Atomic-scale STEM images of the oriented attachment between two $\beta$ $\mathrm{MnO}_{2}$ nanowires. (a) $\mathrm{An} \mathrm{ABF}$ cross-section image of two attached $\beta-\mathrm{MnO}_{2}$ nanowires viewed along the [001] tunnel direction. (b) Atomic scale HAADF image of (100) line defects inside the $\beta-\mathrm{MnO}_{2}$ cross-section. The inset panel shows the corresponding atomic model. (c) An atomic model illustrating the line defects resulted from a 'sliding' effect along the (100) plane. 


\subsubsection{Facet evolution of the $\beta-\mathrm{MnO}_{2}$ microrods}

$\beta-\mathrm{MnO}_{2}$ microrods were synthesized following a typical hydrothermal method reported elsewhere ${ }^{26}$. Both $\mathrm{MnO}_{4}^{-}$and $\mathrm{Mn}^{2+}$ were used as the manganese source and the redox reaction took place between these two ions. Extra $\mathrm{K}^{+}$ions were introduced to regulate the growth of the microrods. Obtained micro rods showed bipyramid prism morphology. The diameter was around $3 \mu \mathrm{m}$ and a length was around 1 5 $\mu \mathrm{m}$ (Figure 4-4). The corresponding XRD patterns confirmed the microrods are all single-phase beta $\mathrm{MnO}_{2}$ and the diffraction peaks fit well with the standard JCPDS 24-0735 (Figure S4-4).
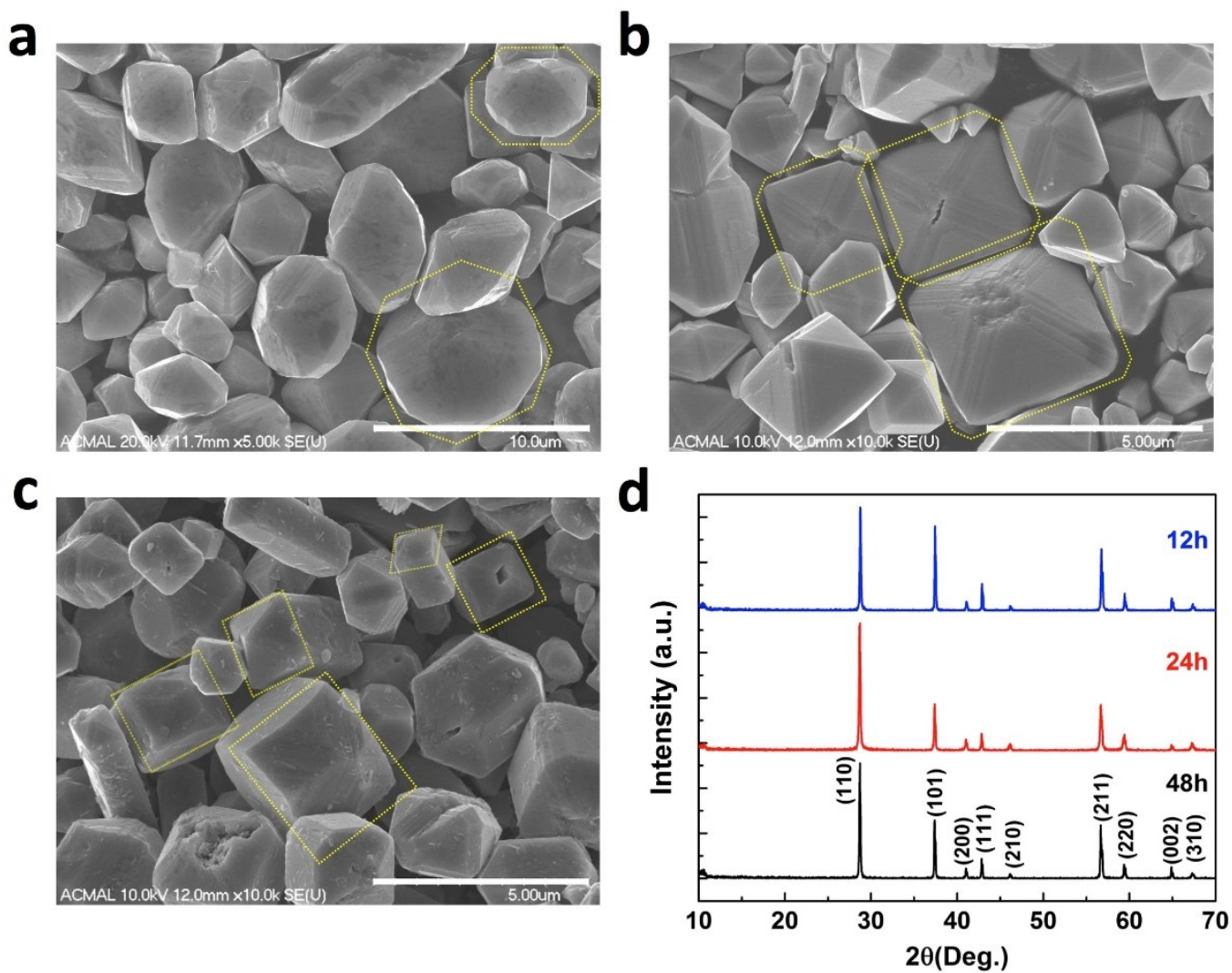

Figure 4-4 Morphology and phase characterization of the synthesized $\beta-\mathrm{MnO}_{2}$ microrods. SEM images of the $\beta-\mathrm{MnO}_{2}$ microrods synthesized at $180{ }^{\circ} \mathrm{C}$ for a reaction 
time of (a) 12h, (b) 24h, and (c) 48h, respectively. Yellow dashed boxes were drawn for better comparison. (d) The corresponding XRD patterns of the microrods obtained at different reaction time.

All the microrods have regular crisscross line defects penetrating both the bipyramid ends and the prism body, which appears to be a symmetrical lateral attachment of four individual microrods. When the hydrothermal reaction time was increased, an evolution of the lateral surfaces can be observed among the facets of the prism part. Under the reaction time of $12 \mathrm{~h}$, the cross-sections of the prism body were mainly octagonalshaped, with a comparable amount of $\{110\}$ and $\{100\}$ facets (confirmed by HRTEM, Figure 4-6a,b). When the reaction time was increased to $48 \mathrm{~h}$, the lateral surfaces of the prism body gradually changed to square-shaped, with an elimination of the cornered $\{100\}$ facets. This surface evolution process was similar to that observed from the $\beta-\mathrm{MnO}_{2}$ nanowires. Along with the increase of the hydrothermal reaction time from $12 \mathrm{~h}$ to $48 \mathrm{~h}$, the integrated XRD peak intensity ratio $\mathrm{I}_{(110)} / \mathrm{I}_{(200)}$ increased from 10.032 to 11.232 (Figure 44d), which shall indicate an increased occupancy of the $\{110\}$ facets and a reduced amount of $\{100\}$ facets in the $\beta-\mathrm{MnO}_{2}$ microrods. 

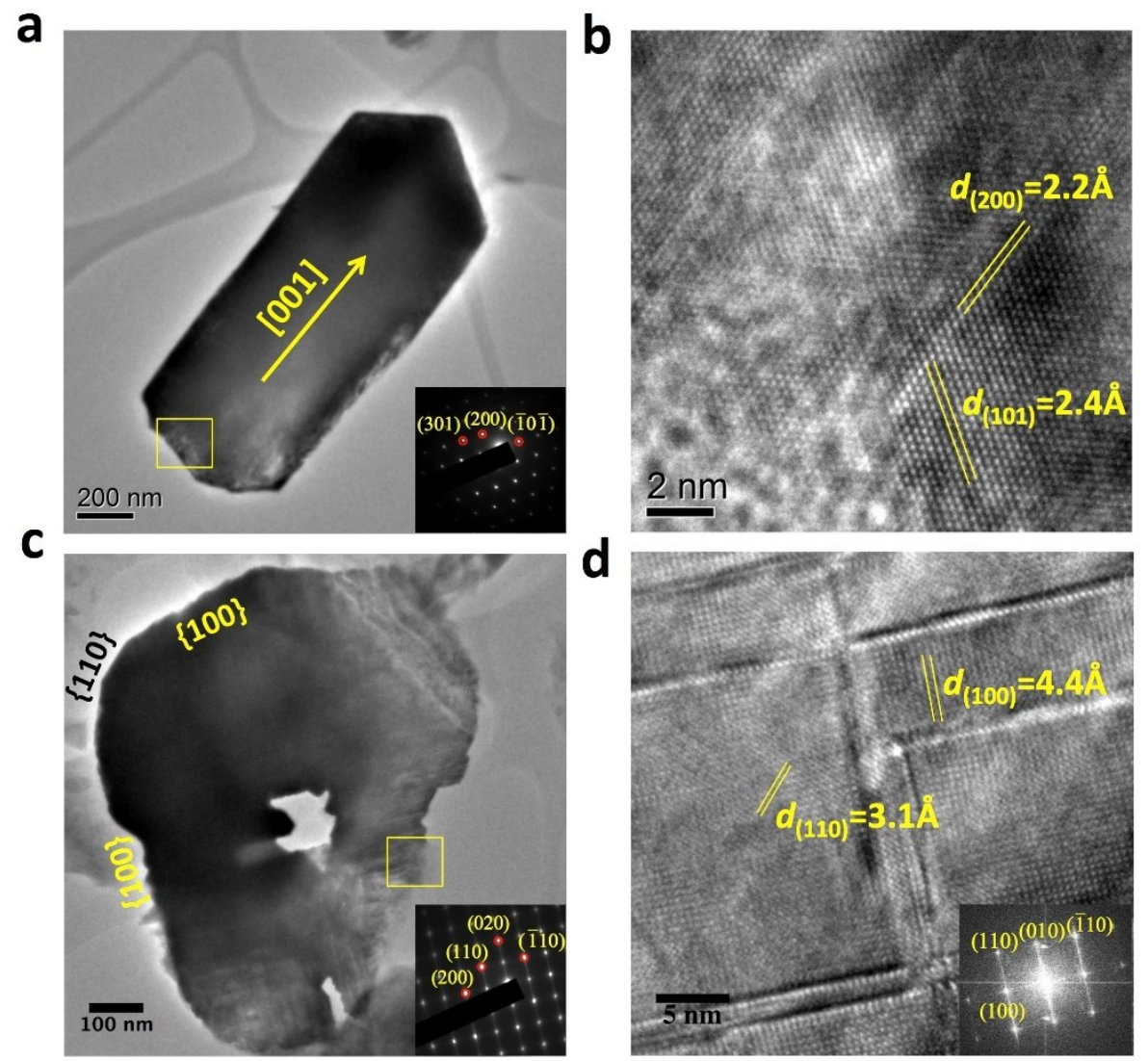

Figure 4-5 HRTEM analysis of the internal structure of the $\beta-\mathrm{MnO}_{2}$ microrods. (a) TEM image of a $\beta-\mathrm{MnO}_{2}$ microrod observed at the [010] zone axis. The inset panel gives the corresponding selected area diffraction (SAED) pattern. (b) HRTEM image obtained at the yellow dashed box in Fig. 5a. (c) A representative cross-section image of the $\beta-\mathrm{MnO}_{2}$ microrods obtained at the [001] zone axis. The inset panel shows the corresponding diffraction pattern. (d) HRTEM image obtained at the highlighted yellow box in Fig. 5c. The inset panel gives the corresponding FFT image.

The lateral surfaces and the internal structure of the prism part were further identified based on a cross-section analysis of the microrods. The growth direction of the $\beta-\mathrm{MnO}_{2}$ microrods was revealed to be along the [001] tunnel direction (Figure 4-5a), which is the same with the $\beta-\mathrm{MnO}_{2}$ nanowires. The HRTEM image obtained from the lateral side 
indicated no lattice mismatch along the line defects viewed from the [010] zone axis (Figure 4-5b). The cross-section analysis in Figure 4-5c further confirmed the lateral surfaces of the $\beta-\mathrm{MnO}_{2}$ microrod consisted of both $\{110\}$ and $\{100\}$ facets, which is also the same with the nanowires. The regular crisscross line defects were clearly revealed in Figure 4-5d. These line defects were also generated along the less stable $\{100\}$ planes and maintained after $48 \mathrm{~h}$ of reaction. Moreover, most of the microrods were likely to be formed based on an oriented symmetrical attachment of four microrods along their mutual $\{100\}$ facets, in order to reduce the overall surface energies. This four-fold symmetrical attachment was also observed in $\beta-\mathrm{MnO}_{2}$ nanowires (Figure S4-5). Figure S4-5a gives a cross-section image of four nanowires attached along their $\{100\}$ facets. Figure S4-5b shows a $200 \mathrm{~nm} \beta-\mathrm{MnO}_{2}$ nanowire with similar attached structure viewed from the lateral side. 

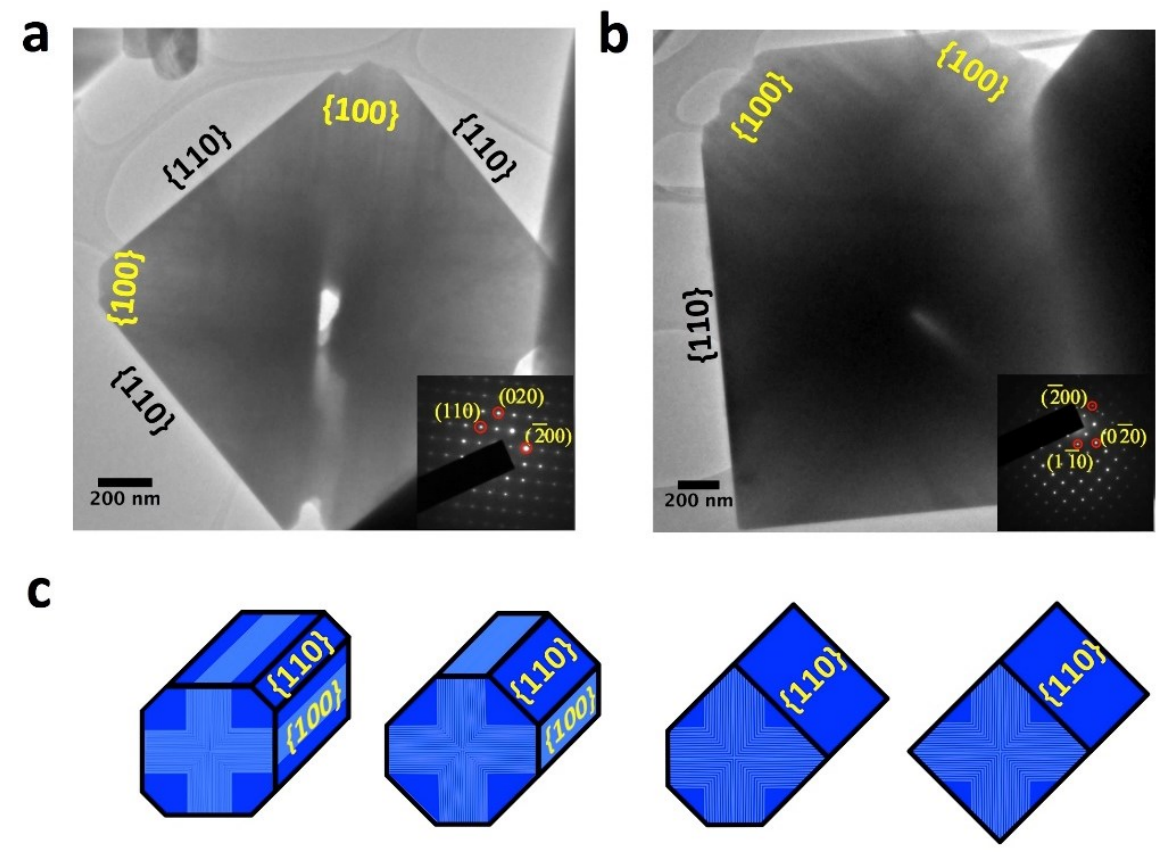

Surface energy driven growth of $\beta-\mathrm{MnO}_{2}$ microrods

Figure 4-6 TEM analysis of the $\beta-\mathrm{MnO}_{2}$ microrods at the intermediate state of growth. Low magnification TEM images of two $\beta-\mathrm{MnO}_{2}$ microrods with (a) four and (b) two $\{100\}$ facets viewed along the [001] zone axis. Insets of panels give the corresponding SAED pattern. (c) Proposed surface-energy-driven growth of $\beta-\mathrm{MnO}_{2}$ microrods with the evolution of the lateral surfaces.

To develop the understanding of the lateral surfaces evolution, the $\beta-\mathrm{MnO}_{2}$ microrods obtained at the reaction time of $12 \mathrm{~h}$ were studied to reveal the possible morphology of the microrods at the intermediate state of evolution. Several microrods were examined and Figure 4-6 gives two representative microrods with different occupancy of $\{100\}$ facets, which shall represent the microrods that are at the intermediate state of surface evolution. Both microrods were analyzed along their [001] tunnel directions. Figure 4-6a shows the $\{100\}$ facets occupied the four corners, which account for around $17.5 \%$ 
of the overall lateral surfaces, calculated roughly based on the length of the intersection in the cross-section image. Figure $4-6 \mathrm{~b}$ shows another microrod with its $\{100\}$ facets taking only two corners. The occupancy of $\{100\}$ facets reduced to $13.5 \%$. Both microrods shall represent the intermediate state of growth for the $\beta-\mathrm{MnO}_{2}$ microrods. The surface evolution process was proposed in Figure 4-6c. Based on the different surface energy of the two kinds of facets, with the increase of the hydrothermal reaction time, the occupancy of the less stable $\{100\}$ facets was reduced, and the cross-section of the prism part changed from octagonal-shaped to square-shaped.

Based on the surface evolution as a function of hydrothermal reaction time and $\mathrm{XRD} / \mathrm{TEM}$ analysis, it can be concluded that the surface-energy-driven mechanism dominated the evolution of both $\beta-\mathrm{MnO}_{2}$ nanowires and microrods. The $\{100\}$ and $\{110\}$ are dominant facets among the lateral surfaces for both morphologies. During the surface evolution process, occupancy of the $\{100\}$ facets was reduced through both self-growth and oriented attachment of the nanowires/micro-rods to reduce the overall surface energies.

The observed surface evolution mechanism is highly informative for facetcontrolled growth of $\beta-\mathrm{MnO}_{2}$ materials. If the occupancy of $\{110\} /\{100\}$ facets can be controlled precisely by optimizing the parameters used for the hydrothermal synthesis, the performance of this materials can be regulated to meet different requirements or working conditions. Compared with the $\{110\}$ surface, the $\{100\}$ surface has higher surface energy and more open serrated structure, which may offer more active sites for surface adsorption or chemical reactions. A different occupancy of these two facets would bring different performance of this material as catalysts or electrode materials for energy storage. 


\subsection{Conclusions}

In summary, the surface-energy-driven mechanism was found to be the dominant mechanism for the lateral surface evolution of both $\beta-\mathrm{MnO}_{2}$ nanowires and microrods synthesized via different precursors. Among the lateral surfaces of both materials, $\{100\}$ and $\{110\}$ facets were found to be the dominant facets. The overall surface energy was reduced through two ways during the surface evolution: (1) self-growth, which was realized by the direct growth of the $\beta-\mathrm{MnO}_{2}$ nanowire or microrod to on the less stable $\{100\}$ facets; (2) oriented attachment, which was based on the attachment of several $\beta$ $\mathrm{MnO}_{2}$ nanowires or microrods along their mutual $\{100\}$ facets. A four-fold oriented attachment was found to dominate the growth of the $\beta-\mathrm{MnO}_{2}$ microrods. During the oriented attachment of both $\beta-\mathrm{MnO}_{2}$ nanowires and microrods, high-density defects were generated along the attached $\{100\}$ planes. The defects appeared to be screw dislocations with $1 / 2<100>$ Burgers vector. The revealed surface evolution phenomena can serve as the guidance for controlled synthesis of $\beta-\mathrm{MnO}_{2}$ nanowires and microrods with unique facets for their wide applications in the field of rechargeable batteries, supercapacitors, catalysts for metal-air batteries and water treatment, etc.

\subsection{References}

1 Jiang, H., Zhao, T., Ma, J., Yan, C. \& Li, C. Ultrafine manganese dioxide nanowire network for high-performance supercapacitors. Chemical Communications 47, 1264-1266 (2011).

2 Huang, X. et al. Highly crystalline macroporous $\beta-\mathrm{MnO}_{2}$ : hydrothermal synthesis and application in lithium battery. Electrochimical Acta 55, 4915-4920 (2010). 
3 Chen, W.-M. et al. Controllable synthesis of hollow bipyramid $\beta-\mathrm{MnO}_{2}$ and its high electrochemical performance for lithium storage. ACS Applied Materials \& Interfaces 4, 3047-3053 (2012).

4 Thapa, A. K., Hidaka, Y., Hagiwara, H., Ida, S. \& Ishihara, T. Mesoporous $\beta$ $\mathrm{MnO}_{2}$ air electrode modified with Pd for rechargeability in lithium-air battery. Journal of Electrochemical Society 158, A1483-A1489 (2011).

5 Zhang, Y., Chen, L., Zheng, Z. \& Yang, F. A redox-hydrothermal route to $\beta$ $\mathrm{MnO}_{2}$ hollow octahedra. Solid State Sciences 11, 1265-1269 (2009).

6 Boppana, V. B. R. \& Jiao, F. Nanostructured $\mathrm{MnO}_{2}$ : an efficient and robust water oxidation catalyst. Chemical Communications 47, 8973-8975 (2011).

$7 \quad$ Huang, Z. et al. Silver decorated beta-manganese oxide nanorods as an effective cathode electrocatalyst for rechargeable lithium-oxygen battery. Journal of Alloys and Compounds 626, 173-179 (2015).

8 Dong, Y., Yang, H., He, K., Song, S. \& Zhang, A. $\beta-\mathrm{MnO}_{2}$ nanowires: a novel ozonation catalyst for water treatment. Applied Catalysis B: Environmental 85, 155-161 (2009).

9 Ghodbane, O., Pascal, J.-L. \& Favier, F. Microstructural effects on charge-storage properties in $\mathrm{MnO}_{2}$-based electrochemical supercapacitors. ACS Applied Materials \& Interfaces 1, 1130-1139 (2009).

10 Zang, J. \& Li, X. In situ synthesis of ultrafine $\beta-\mathrm{MnO}_{2} /$ polypyrrole nanorod composites for high-performance supercapacitors. Journal of Materials Chemistry 21, 10965-10969 (2011).

11 Cheng, F. et al. Facile controlled synthesis of $\mathrm{MnO}_{2}$ nanostructures of novel shapes and their application in batteries. Inorganic Chemistry 45, 2038-2044 (2006).

12 Luo, J.-Y., Zhang, J.-J. \& Xia, Y.-Y. Highly electrochemical reaction of lithium in the ordered mesoporosus $\beta-\mathrm{MnO}_{2}$. Chemistry of Materials 18, 5618-5623 (2006).

13 Jiao, F. \& Bruce, P. G. Mesoporous crystalline $\beta-\mathrm{MnO}_{2}-\mathrm{a}$ reversible positive electrode for rechargeable lithium batteries. Advanced Materials 19, 657-660 (2007).

14 Zheng, D., Yin, Z., Zhang, W., Tan, X. \& Sun, S. Novel branched $\gamma$-MnOOH and $\beta-\mathrm{MnO}_{2}$ multipod nanostructures. Crystal Growth \& Design 6, 1733-1735 (2006). 
15 Zhou, J. et al. $\mathrm{MnO}_{2}$ nanosheet-assisted hydrothermal synthesis of $\beta-\mathrm{MnO}_{2}$ branchy structures. Materials Letters 79, 288-291 (2012).

16 Oxford, G. A. \& Chaka, A. M. First-principles calculations of clean, oxidized, and reduced $\beta-\mathrm{MnO}_{2}$ surfaces. Journal of Physical Chemistry C 115, 16992-17008 (2011).

17 Tompsett, D. A., Parker, S. C., Bruce, P. G. \& Islam, M. S. Nanostructuring of $\beta$ $\mathrm{MnO}_{2}$ : The important role of surface to bulk ion migration. Chemistry of Materials 25, 536-541 (2013).

18 Oxford, G. A. \& Chaka, A. M. Structure and stability of hydrated $\beta-\mathrm{MnO}_{2}$ surfaces. Journal of Physical Chemistry C 116, 11589-11605 (2012).

19 Tompsett, D. A., Parker, S. C. \& Islam, M. S. Rutile ( $\beta$-) $\mathrm{MnO}_{2}$ surfaces and vacancy formation for high electrochemical and catalytic performance. Journal of the American Chemical Society 136, 1418-1426 (2014).

20 Tian, N., Zhou, Z.-Y., Sun, S.-G., Ding, Y. \& Wang, Z. L. Synthesis of tetrahexahedral platinum nanocrystals with high-index facets and high electrooxidation activity. Science 316, 732-735 (2007).

21 Zhang, B. et al. Facet-dependent catalytic activity of platinum nanocrystals for triiodide reduction in dye-sensitized solar cells. Scientific Report 3, 1836 (2013).

22 Tian, N., Zhou, Z.-Y., Yu, N.-F., Wang, L.-Y. \& Sun, S.-G. Direct electrodeposition of tetrahexahedral Pd nanocrystals with high-index facets and high catalytic activity for ethanol electrooxidation. Journal of the American Chemical Society 132, 7580-7581 (2010).

23 Ming, T. et al. Growth of tetrahexahedral gold nanocrystals with high-index facets. Journal of the American Chemical Society 131, 16350-16351 (2009).

24 Chen, J. S. et al. Constructing hierarchical spheres from large ultrathin anatase $\mathrm{TiO}_{2}$ nanosheets with nearly $100 \%$ exposed (001) facets for fast reversible lithium storage. Journal of the American Chemical Society 132, 6124-6130 (2010).

25 Gao, R. et al. Facet-dependent electrocatalytic performance of $\mathrm{Co}_{3} \mathrm{O}_{4}$ for rechargeable Li-O 2 battery. Journal of Physical Chemistry C 119, 4516-4523 (2015).

26 Huang, X., Lv, D., Yue, H., Attia, A. \& Yang, Y. Controllable synthesis of $\alpha$-and $\beta-\mathrm{MnO}_{2}$ : cationic effect on hydrothermal crystallization. Nanotechnology $\mathbf{1 9}$, 225606 (2008). 
27 Chen, N. et al. Ionic liquid-assisted hydrothermal synthesis of $\beta-\mathrm{MnO}_{2}$ with hollow polyhedra morphology. Colloids and Surfaces A: Physicochemical and Engineering Aspects 387, 10-16 (2011).

28 Wang, X. \& Li, Y. Selected-control hydrothermal synthesis of $\alpha$-and $\beta-\mathrm{MnO}_{2}$ single crystal nanowires. Journal of the American Chemical Society 124, 28802881 (2002).

29 Wang, G., Tang, B., Zhuo, L., Ge, J. \& Xue, M. Facile and selected-control synthesis of $\beta-\mathrm{MnO}_{2}$ nanorods and their magnetic properties. European Journal of Inorganic Chemistry 2006, 2313-2317 (2006).

30 Zheng, D. et al. One-step preparation of single-crystalline $\beta-\mathrm{MnO}_{2}$ nanotubes. Journal of Physical Chemistry B 109, 16439-16443 (2005).

31 Wang, X. \& Li, Y. Synthesis and formation mechanism of manganese dioxide nanowires/nanorods. Chemistry-A European Journal 9, 300-306 (2003).

32 Zhang, X., Yang, W., Yang, J. \& Evans, D. G. Synthesis and characterization of $\alpha-\mathrm{MnO}_{2}$ nanowires: self-assembly and phase transformation to $\beta-\mathrm{MnO}_{2}$ microcrystals. Journal of Crystal Growth 310, 716-722 (2008).

$33 \mathrm{Su}, \mathrm{D} ., \mathrm{Ahn}, \mathrm{H} .-\mathrm{J}$. \& Wang, G. $\beta-\mathrm{MnO}_{2}$ nanorods with exposed tunnel structures as high-performance cathode materials for sodium-ion batteries. NPG Asia Materials 5, e70 (2013).

34 Yuan, Y. et al. Atomistic insights into the oriented attachment of tunnel-based oxide nanostructures. ACS Nano 10, 539-548 (2015). 


\section{Chapter 5 Cations controlled growth of $\beta-\mathrm{MnO}_{2}$ crystals with tunable facets for electrochemical energy storage ${ }^{3}$}

Engineering crystal facets to enhance their functionalities often require complex processing routes to suppress the growth of surfaces with the lowest thermodynamic energies. Herein, we report a unique method to control the morphologies of $\beta-\mathrm{MnO}_{2}$ crystals with different occupancy of $\{100\} /\{111\}$ facets through the effect of $\mathrm{K}^{+}$cations. Combining aberrationcorrected scanning transmission electron microscopy (STEM), ultramicrotomy, and dynamic functional theory (DFT) simulation, we clarified that the $\beta-\mathrm{MnO}_{2}$ crystals were formed through a direct solid-state phase transition process. Increasing the concentration of $\mathrm{K}^{+}$cations in the precursor gradually changed the morphology of $\beta-\mathrm{MnO}_{2}$ from bipyramid prism $(\{100\}+\{111\}$ facets) to an octahedron structure ( $\{111\}$ facets). The $\mathrm{K}^{+}$cations controlled the morphology of $\beta-\mathrm{MnO}_{2}$ by affecting the formation of $\alpha$ $\mathrm{K}_{0.5} \mathrm{Mn}_{4} \mathrm{O}_{8}$ intermediate phase and the subsequent phase transition. Utilizing the $\beta$ $\mathrm{MnO}_{2}$ crystals as the cathode for Li-ion batteries showed that highly exposed $\{111\}$ facets offered $\beta-\mathrm{MnO}_{2}$ crystal better rate performance, with $\sim 70 \%$ capacity retention when the charge-discharge rate increased from $20 \mathrm{~mA} / \mathrm{g}$ to $200 \mathrm{~mA} / \mathrm{g}$. Our work revealed a new mechanism to tune the morphology of this earth-abundant metal oxide crystal, which could be used to adjust its electrochemical performance for different applications, such as supercapacitors and catalysts for metal-air batteries and fuel cells.

${ }^{3}$ The material contained in this chapter was previously published in Nano Energy. (Reprinted with permission from Yao, W., Odegard, G., Huang, Z., Yuan, Y., AsayeshArdakani H., ... Amine, K., Lu, J., \& Shahbazian-Yassar, R. Nano Energy, 2018, 48, 301311. Copyright 2018, Elsevier.) 


\subsection{Introduction}

Manganese dioxide $\left(\mathrm{MnO}_{2}\right)$ has been widely used in energy storage systems, either as an electrode material for rechargeable batteries and supercapacitors or as a bifunctional catalyst for oxygen reduction/evolution reactions (ORR/OER) in metal-air batteries and fuel cells. ${ }^{1}$ The crystal structures of $\mathrm{MnO}_{2}$ can be differentiated into $\alpha-, \beta-, \gamma$-, and $\delta$-phases based on how its $\left[\mathrm{MnO}_{6}\right]$ octahedra units are connected. The crystal phases of $\mathrm{MnO}_{2}$ can greatly affect the electrochemical performances. As the cathode material for $\mathrm{Zn}-\mathrm{MnO}_{2}$ and Li- $-\mathrm{MnO}_{2}$ primary batteries, $\gamma-\mathrm{MnO}_{2}$ nanowires delivered a higher capacity than $\alpha-\mathrm{MnO}_{2}$ and $\beta-\mathrm{MnO}_{2}$ nanowires. ${ }^{2,3}$ As the electrode for supercapacitors, the specific capacitances of different $\mathrm{MnO}_{2}$ crystals were found to follow the order: $\alpha \approx \delta>\gamma>\beta .{ }^{4,5}$ Apart from the crystal phases, the morphology or exposed crystal facets also affect the electrochemical performance of $\mathrm{MnO}_{2}{ }^{3,6-8}$ As the cathode for lithium-ion batteries, $\alpha-\mathrm{MnO}_{2}$ nanowires with exposed (210) facets showed higher capacity than the nanowires with (110) facets. ${ }^{9} \alpha$ $\mathrm{MnO}_{2}$ nanotubes exhibited higher stability than its nanowire shape as the cathode catalyst for Li-air batteries. ${ }^{6}$ The (101) surfaces of rutile $\mathrm{MnO}_{2}$ show better catalytic activity for oxygen evolution reaction compared with the (110) surfaces. ${ }^{10}$ To maximize the electrochemical performance of $\mathrm{MnO}_{2}$ crystals for different applications, it is highly needed to control the synthesis of their structures with different phases and morphologies.

Rutile $\beta-\mathrm{MnO}_{2}$ crystal is thermodynamically the most stable phase among the $\mathrm{MnO}_{2}$ polymorphs. ${ }^{11}$ Bulk level $\beta-\mathrm{MnO}_{2}$ is generally undesirable for rechargeable batteries and supercapacitor applications, because of its poor kinetics for lithium intercalation and limited surface areas for charge storage. ${ }^{3}$ However, recent studies showed different 
electrochemical performance of $\beta-\mathrm{MnO}_{2}$ with different morphologies. As the cathode for lithium-ion batteries, mesoporous $\beta-\mathrm{MnO}_{2}$ can deliver ten times higher capacity (284 mAh $\left.\mathrm{g}^{-1}\right)$ than the bulk level counterpart $\left(<20 \mathrm{mAh} \mathrm{g}^{-1}\right) \cdot{ }^{12}$ Hollow bipyramids $\beta-\mathrm{MnO}_{2}$ can deliver up to $150 \mathrm{mAh} \mathrm{g}{ }^{-1} \cdot{ }^{13}$ As the electrode for supercapacitors, ultrafine $\beta-\mathrm{MnO}_{2}$ nanowires with high aspect ratios and small diameters $(<10 \mathrm{~nm})$ gave a reported specific capacitance around $294 \mathrm{~F} \cdot \mathrm{g}^{-1}$ at $1 \mathrm{~A} \cdot \mathrm{g}^{-1}$, which is also ten times higher than that of $\beta-\mathrm{MnO}_{2}$ nanorods $\left(28 \mathrm{~F} \cdot \mathrm{g}^{-1}\right) \cdot{ }^{4,14}$ These observations indicate that it is possible to greatly improve the electrochemical performance of $\beta-\mathrm{MnO}_{2}$ crystal by controlling its morphology or exposed surfaces.

Hydrothermal synthesis is commonly used for the synthesis of well-distributed $\mathrm{MnO}_{2}$ nanostructures. ${ }^{15-17}$ The molar ratio of precursors, concentration of surfactants, temperature, and hydrothermal reaction time were mainly used to regulate the phase and morphology of the obtained $\mathrm{MnO}_{2}$ structures. ${ }^{15,17}$ Several cations $\left(\mathrm{K}^{+}, \mathrm{NH}_{4}^{+}, \mathrm{H}^{+}\right)$and anions were found to be able to regulate the phase of $\mathrm{MnO}_{2}$ crystals during synthesis. ${ }^{18-21}$ Among these ions, the $\mathrm{K}^{+}$ion can stabilize the [2×2] tunnels in $\alpha-\mathrm{MnO}_{2}$ or the interlayer spaces in $\delta-\mathrm{MnO}_{2}$ and it was generally used for the synthesis of $\alpha-\mathrm{K}_{\mathrm{x}} \mathrm{MnO}_{2}$ nanostructures. ${ }^{22}$ When the concentration of $\mathrm{K}^{+}$ions in the precursor increased, there was a general trend of phase shift from $\beta-\mathrm{MnO}_{2}$ to $\alpha-\mathrm{MnO}_{2}$ and then $\delta-\mathrm{MnO}_{2}{ }^{16,23}$ Previous studies on the effect of $\mathrm{K}^{+}$ion mainly focused on its effect on the phase transition during the synthesis. ${ }^{19,24}$ The study of $\mathrm{K}^{+}$cationic effect on the morphology of $\mathrm{MnO}_{2}$ structures, however, is limited. 
In this work, $\beta-\mathrm{MnO}_{2}$ crystals with bipyramid prism structures were synthesized using a hydrothermal method. Ex-situ studies showed the $\beta-\mathrm{MnO}_{2}$ microstructure was formed through an oriented attachment and subsequent solid-state phase transition from $\alpha$ $\mathrm{K}_{0.5} \mathrm{Mn}_{4} \mathrm{O}_{8}$ nanowires. $\beta-\mathrm{MnO}_{2}$ crystals with tunable morphologies were realized by controlling the concentration of $\mathrm{KCl}$ precursors. Combining field emission scanning electron microscopy (FE-SEM), X-ray diffraction (XRD), transmission electron microscopy (TEM) analysis, and density functional theory (DFT) simulation, $\mathrm{K}^{+}$cations were found to affect the morphology of $\beta-\mathrm{MnO}_{2}$ crystals through affecting the formation of $\alpha-\mathrm{K}_{0.5} \mathrm{Mn}_{4} \mathrm{O}_{8}$ intermediate phase and the following phase transition process. To further reveal the effect of crystal facets on the electrochemical performance of $\beta-\mathrm{MnO}_{2}$, the crystal structures with different occupancy of $\{100\}$ and $\{111\}$ facets was tested as the cathode for Li-ion batteries.

\subsection{Results and Discussion}

\subsubsection{Growth mechanism of $\beta-\mathrm{MnO}_{2}$ crystals}

$\beta-\mathrm{MnO}_{2}$ crystals were synthesized based on the redox reaction between $\mathrm{KMnO}_{4}$ and $\mathrm{MnCl}_{2}$ $\cdot \mathrm{H}_{2} \mathrm{O}$, with $0.05 \mathrm{M} \mathrm{KCl}$ as the surfactant. Figure 5-1 a-d shows the FE-SEM images and XRD patterns of the $\mathrm{MnO}_{2}$ structure synthesized at different reaction times. The $\mathrm{MnO}_{2}$ structures obtained after $1.0 \mathrm{~h}$ and $2.0 \mathrm{~h}$ of hydrothermal reactions were mainly indexed to be $\alpha-\mathrm{MnO}_{2}$ phase (JCPDS 44-0141, $a=b=9.78 \AA, c=2.86 \AA$ ). A small amount of plateshaped $\gamma-\mathrm{MnO}_{2}$ phase (JCPDS 14-0644, $a=6.36 \AA, b=10.15 \AA, c=4.09 \AA$ ) was also detected. Previous studies demonstrated that $\mathrm{K}^{+}$ions can act as the stabilizer for the $[2 \times 2]$ tunnels and increasing the concentration of $\mathrm{K}^{+}$ions would facilitate the formation of $\alpha$ - 
$\mathrm{MnO}_{2}$ crystals. ${ }^{18,25}$ Energy-dispersive X-ray spectroscopy (EDS) analysis confirmed that the $\alpha-\mathrm{MnO}_{2}$ nanowires were stabilized with $\mathrm{K}^{+}$cations, with a chemical composition close to $\alpha-\mathrm{K}_{0.5} \mathrm{Mn}_{4} \mathrm{O}_{8}$ (Fig. S5-1). Under the reaction time of $4.0 \mathrm{~h}, \beta-\mathrm{MnO}_{2}$ crystals started to form (Fig. 5-1b, indicated by the red arrow). After $6.0 \mathrm{~h}$, most of the nanowires transformed to micro-size $\mathrm{MnO}_{2}$. The XRD patterns were in good agreement with $\beta-\mathrm{MnO}_{2}$ (JCPDS No. 24-0735) with lattice parameters $a=b=4.399 \AA, c=2.874 \AA$. The size of the $\mathrm{MnO}_{2}$ structure clearly increased during the phase transition from the $\alpha-\mathrm{MnO}_{2}$ nanowires to $\beta-\mathrm{MnO}_{2}$ microrods. This phase transformation was previously proposed to be a combination of oriented attachment and dissolution-recrystallization process. ${ }^{26}$ The $\beta-\mathrm{MnO}_{2}$ crystals showed bipyramid prism morphologies, with clear crisscross defects across the prism body, which may result from the oriented attachment of $\alpha-\mathrm{MnO}_{2}$ nanowires. The redox reaction between $\mathrm{KMnO}_{4}$ and $\mathrm{MnCl}_{2}$ generated $\mathrm{HCl}$, which increased the acidity of the solution (Equation 5-1).

$$
2 \mathrm{KMnO}_{4}+3 \mathrm{MnCl}_{2}+2 \mathrm{H}_{2} \mathrm{O} \rightarrow 5 \mathrm{MnO}_{2}+2 \mathrm{KCl}+4 \mathrm{HCl}
$$

The acidity of the solution facilitated the removal of the $\mathrm{K}^{+}$ions in the [2×2] tunnels of $\alpha$ $\mathrm{MnO}_{2}{ }^{27}$ Under the high reaction temperature of $180{ }^{\circ} \mathrm{C}, \quad \beta-\mathrm{MnO}_{2}$ becomes thermodynamically more stable, therefore, the $\alpha-\mathrm{MnO}_{2}$ nanowires will undergo an oriented attachment and subsequent phase transition to $\beta-\mathrm{MnO}_{2}$ microstructures (Fig. 5-1e). ${ }^{24,26}$ 

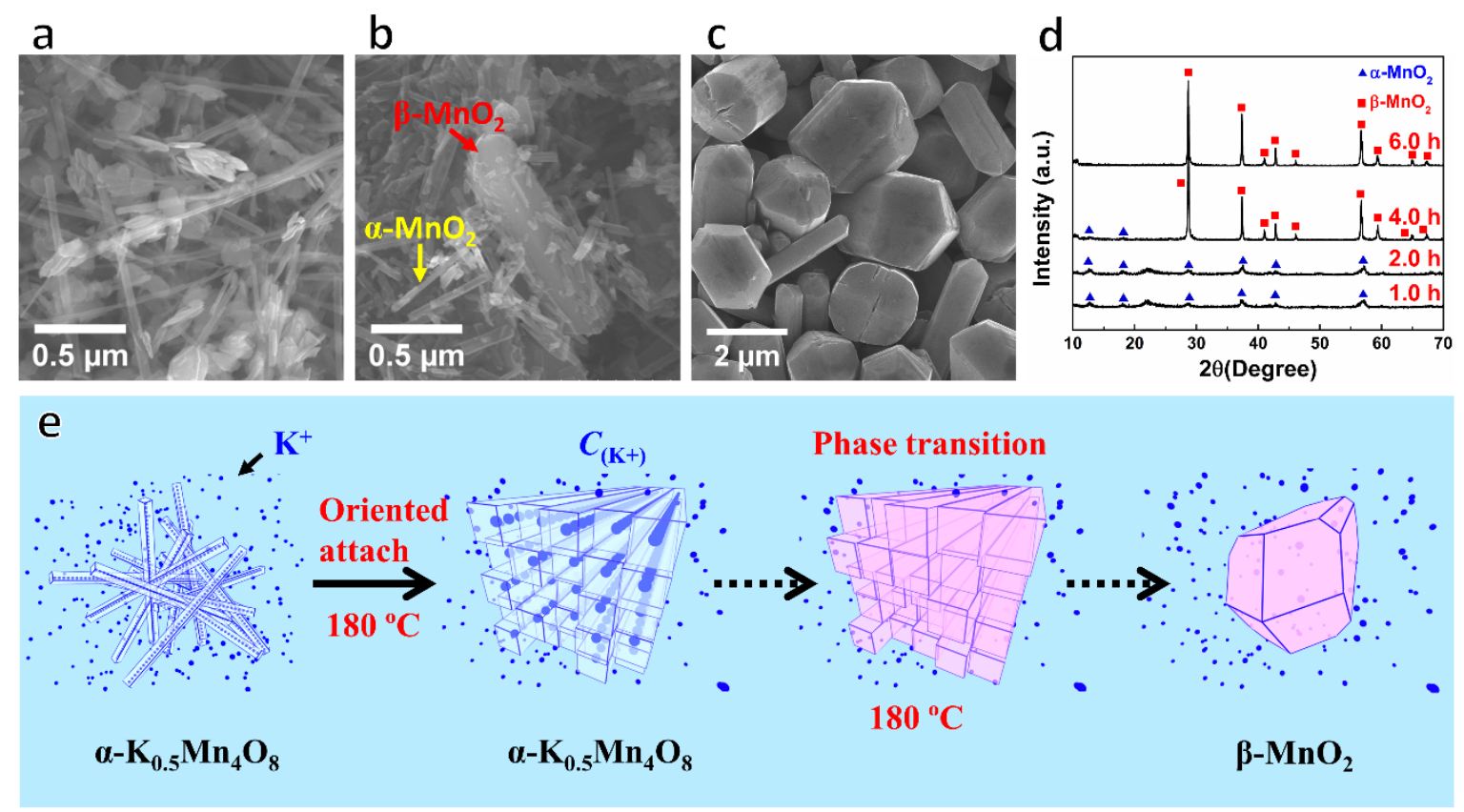

Figure 5-1 (a-c) FE-SEM images of the $\mathrm{MnO}_{2}$ obtained with $0.05 \mathrm{M} \mathrm{KCl}$ under different hydrothermal reaction time of (a) $2.0 \mathrm{~h}$; (b) $4.0 \mathrm{~h}$; (c) $6.0 \mathrm{~h}$. (d) XRD patterns of the $\mathrm{MnO}_{2}$ obtained under different reaction times. (e) Schematic image illustrating the oriented attachment and phase transition processes. The $\mathrm{K}^{+}$stabilizing cations are expelled from the crystal structure during the phase transition. The solid arrow indicates the observed process, while the dashed arrows indicate the proposed process.

Although previous work has observed the oriented attachment of the $\alpha-\mathrm{MnO}_{2}$ nanowires, ${ }^{24}$ the phase transition process is still unknown. To better understand this process, an in-depth analysis of the crisscross defects was performed using ultramicrotomy and aberration-corrected STEM (Fig. 5-2). The low-angle annular dark-field (LAADF) images viewed along the [001] zone axis of $\beta-\mathrm{MnO}_{2}$ cross-sections (Fig. 5-2a, b) showed crisscross defects within the bipyramid prism structure. The density of defects was higher around the corner of the structure than the interior, which may indicate that the interior phase transition was more developed than the surfaces. This indicate the oriented 
attachment and phase transition process could be happening consecutively at the same time instead of two separated processes. Atomic-scale high-angle annular dark-field (HAADF) image of the cross-section sample (Fig. 5-2c) showed clearly [2×2] tunnels and [1×1] tunnels of $\mathrm{MnO}_{2}$, suggesting a direct solid-state phase transition from $\alpha-\mathrm{MnO}_{2}$ to $\beta-\mathrm{MnO}_{2}$. Researchers previously proposed this phase transition to be the dissolutionrecrystallization mechanism, based on the fact that alpha $\mathrm{MnO}_{2}$ has [2×2] tunnel structure but beta $\mathrm{MnO}_{2}$ contains only [1×1] tunnel structure and the morphology changes a lot after phase transition. ${ }^{26,28,29}$ However, following the dissolution-recrystallization mechanism, [2×2] tunnels are not supposed to be detected in the cross-section of beta $\mathrm{MnO}_{2}$. This direct solid-state phase transition is also consistent with recent in situ powder X-ray diffraction observations, where the two phases showed coherence length throughout the reaction stage. ${ }^{30}$ In fact, most of the defects were consisted of [1×2] tunnels of $\left[\mathrm{MnO}_{6}\right]$ octahedron units, and a small amount of [2×2] tunnels can be found around the intersections of these defects. The $[2 \times 2]$ tunnels were combined with $[1 \times 1]$ tunnels through the $[1 \times 2]$ tunnels. The $\mathrm{K}^{+}$stabilizing cations in the $[2 \times 2]$ tunnels were also detected.

Our previous studies showed that the oriented attachment of $\alpha-\mathrm{MnO}_{2}$ nanowires is preferred along the $\{110\}$ planes of the $\alpha-\mathrm{MnO}_{2}$ crystal to form [2×3] tunnels. ${ }^{25}$ During the phase transition from $\alpha-\mathrm{MnO}_{2}$ to $\beta-\mathrm{MnO}_{2}$, these [2×3] interfaces may transfer to two [1×2] tunnels with the extraction of the $\mathrm{K}^{+}$stabilizing ions and the introduction of free $\left[\mathrm{MnO}_{\mathrm{x}}\right]$ units because of their relatively low stability than [2×2] tunnels (Fig. S5-2). The $\{110\}$ planes of $\alpha-\mathrm{MnO}_{2}$ crystal have similar arrangement of $\mathrm{Mn}$ and $\mathrm{O}$ atoms with $\{100\}$ plans of $\beta-\mathrm{MnO}_{2}$ crystal (Fig. S5-3). This explains a relatively large amount of [1×2] tunnels 
were observed along the $\{100\}$ plans of $\beta-\mathrm{MnO}_{2}$ after the phase transition. The [2×2] tunnels may also transform to $[1 \times 2]$ tunnels and then to $[1 \times 1]$ tunnels with the introduction of $\left[\mathrm{MnO}_{\mathrm{x}}\right]$ units (Fig. $\left.5-2 \mathrm{c}\right)$. This direct phase transition from $[2 \times 2]$ to $[1 \times 1]$ also introduce lattice distortions of the $\mathrm{MnO}_{2}$ crystals. Thus, high-stress field was generated along the defective regions (Fig. 5-2d).

To confirm the existence of $\mathrm{K}^{+}$stabilizing ions in the [2×2] tunnels, electron energy loss spectroscopy (EELS) was adopted to check the Mn valence state around different tunnel walls (Fig. 5-2e). The Mn valence state can be differentiated based on the energydifference of $\mathrm{L}_{2,3}$ white-lines $\left(\Delta E\left(\mathrm{~L}_{2}-\mathrm{L}_{3}\right)\right)$ or the white-line intensity ratio $\left(\mathrm{L}_{3} / \mathrm{L}_{2}\right){ }^{31}$ No observable shift was identified for the $\mathrm{Mn} \mathrm{L}_{2}$ and $\mathrm{L}_{3}$ peaks obtained from the [1×1] and [1×2] tunnel walls. However, both the $\mathrm{Mn}_{2}$ and $\mathrm{L}_{3}$ peaks acquired from [2×2] tunnel walls shifted to the low energy side for $0.45 \mathrm{eV}$ and $0.15 \mathrm{eV}$ respectively, indicating a reduced Mn valence state because of the $\mathrm{K}^{+}$ions in the [2×2] tunnel. Further analysis of the white-line intensity ratio $\left(\mathrm{L}_{3} / \mathrm{L}_{2}\right)$ showed that the Mn valence is close to $4^{+}$along the $[1 \times 1]$ and $[1 \times 2]$ tunnels, and around $3.6^{+}$along the $[2 \times 2]$ tunnels (Fig. S5-4). This again demonstrated that the $\beta-\mathrm{MnO}_{2}$ microstructure was formed through a direct solid-state phase transition from $\alpha-\mathrm{K}_{0.5} \mathrm{Mn}_{4} \mathrm{O}_{8}$. 

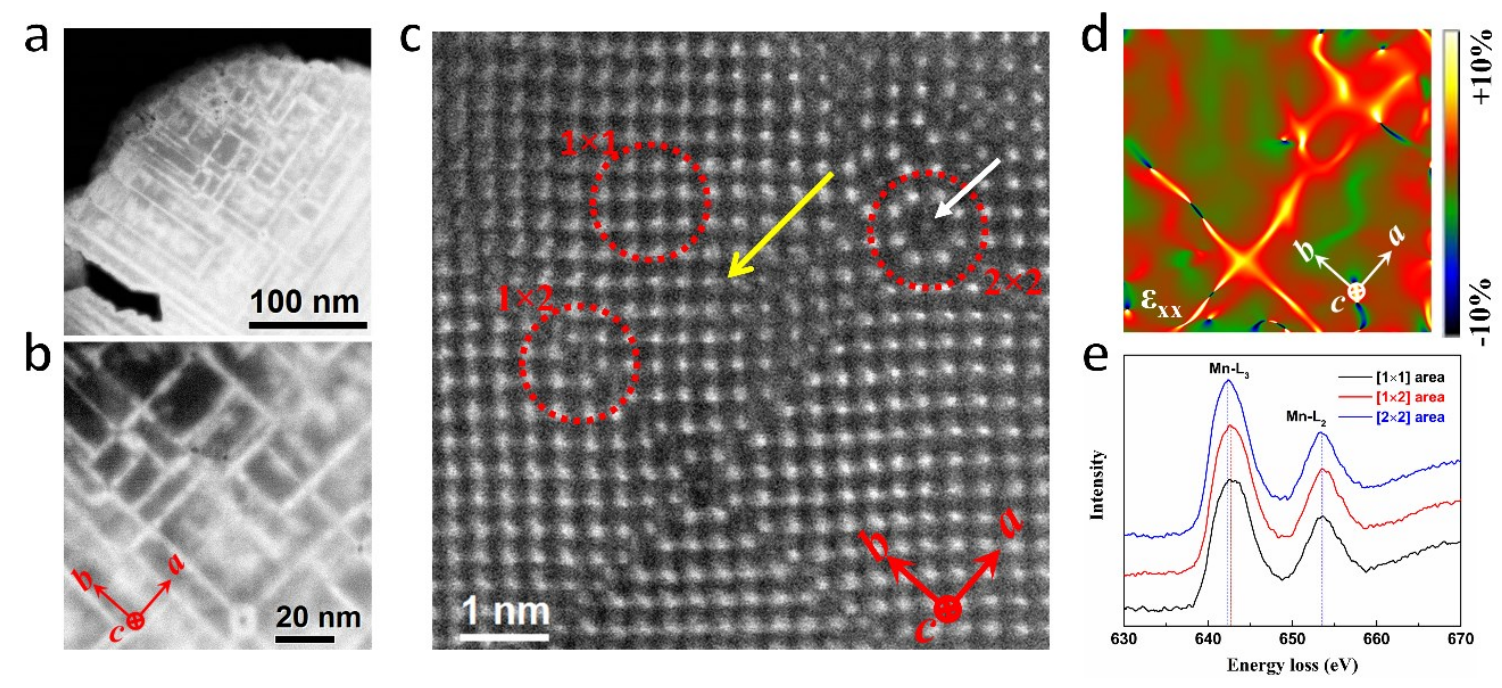

Figure 5-2 $(\mathrm{a}, \mathrm{b}) \mathrm{LAADF}$ images of $\beta-\mathrm{MnO}_{2}$ cross-section sample viewed along the [001] zone axis. (c) Atomic-scale HAADF image showing the transition of [2×2] tunnels to [1×1] tunnels along the defects. The yellow arrow indicates a representative tunnel transition process. The white arrow indicates the location of $\mathrm{K}^{+}$stabilizing ion. (d) $\varepsilon_{x x}$ strain mapping near the $\{100\}$ interfaces showing in Fig. 2c. (e) EELS spectra of $\mathrm{Mn} \mathrm{L}_{2}, \mathrm{~L}_{3}$ peaks obtained from three regions with different tunnel sizes.

\subsection{2 $\mathrm{K}^{+}$cations effect on the morphology of $\beta-\mathrm{MnO}_{2}$ structure}

Previous studies proposed that the $\mathrm{K}^{+}$cations affect the nucleation process through the formation of different crystal phases, where the $\mathrm{K}^{+}$cations were used to stabilize the [2×2] tunnels of the $\alpha-\mathrm{MnO}_{2}$ nuclei or the interlayer space in $\delta-\mathrm{MnO}_{2}$ nanosheets. ${ }^{23}$ The continued crystal growth process was proposed to be controlled by a thermodynamic growth regimes, where 1D growth and oriented attachment dominated the growth process. ${ }^{23,25}$ In our case, the formation of $\beta-\mathrm{MnO}_{2}$ crystals involved the phase transition from $\alpha-\mathrm{K}_{0.5} \mathrm{Mn}_{4} \mathrm{O}_{8}$, consisted of [2×2] tunnels stabilized with $\mathrm{K}^{+}$cations, to [1×1] tunnels. This direct phase transition process involved the extraction of $\mathrm{K}^{+}$stabilizing ions from the structure. If the concentration of $\mathrm{KCl}$ precursors in the solution varies, the extraction of $\mathrm{K}^{+}$ 
ions may be affected because of a concentration gradient along the solid-liquid interfaces. This may in return affect the phase transition process and the morphology of obtained $\beta$ $\mathrm{MnO}_{2}$ structures.

To reveal the effect of $\mathrm{K}^{+}$cations, $\mathrm{KCl}$ precursors with different concentrations (varied from $0.02 \mathrm{M}$ to $0.09 \mathrm{M}$ ) were introduced individually to the redox reaction. SEM was used to examine the morphologies of obtained $\beta-\mathrm{MnO}_{2}$ crystals. With a low concentration of $\mathrm{KCl}$ precursor $(0.02 \mathrm{M})$, the morphology of the synthesized $\mathrm{MnO}_{2}$ showed a bipyramid prism shape with an averaged prism width around $1.2 \mu \mathrm{m}$ and an averaged prism length around $4.8 \mu \mathrm{m}$ (Fig. 5-3a, e). Further increasing the concentration of $\mathrm{KCl}$ precursor gradually changed the bipyramid prism to an octahedron shape (Fig. 5-3b, c, d). When the concentration of $\mathrm{KCl}$ increased to $0.09 \mathrm{M}$, the averaged prism length reduced to $0.8 \mu \mathrm{m}$ and the averaged width increased to $2.7 \mu \mathrm{m}$ (Fig. 5-3e). The obtained $\mathrm{MnO}_{2}$ crystals were confirmed to be $\beta-\mathrm{MnO}_{2}$ crystals through their XRD patterns (Fig. 5-3f). The ratio of integrated XRD peak intensity $\mathrm{I}_{(111)} / \mathrm{I}_{(110)}$ increased from 0.128 to 0.270 when the concentration of $\mathrm{KCl}$ increased from $0.02 \mathrm{M}$ to $0.08 \mathrm{M}$, indicating a preferred surface evolution of the $\beta-\mathrm{MnO}_{2}$ crystals. $^{32}$ 


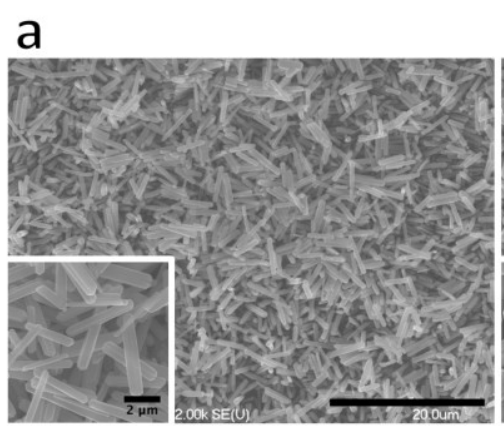

b

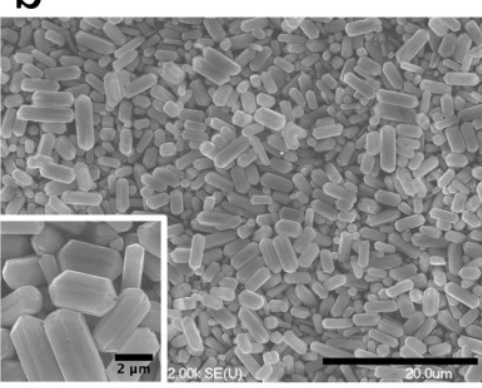

C

d

e
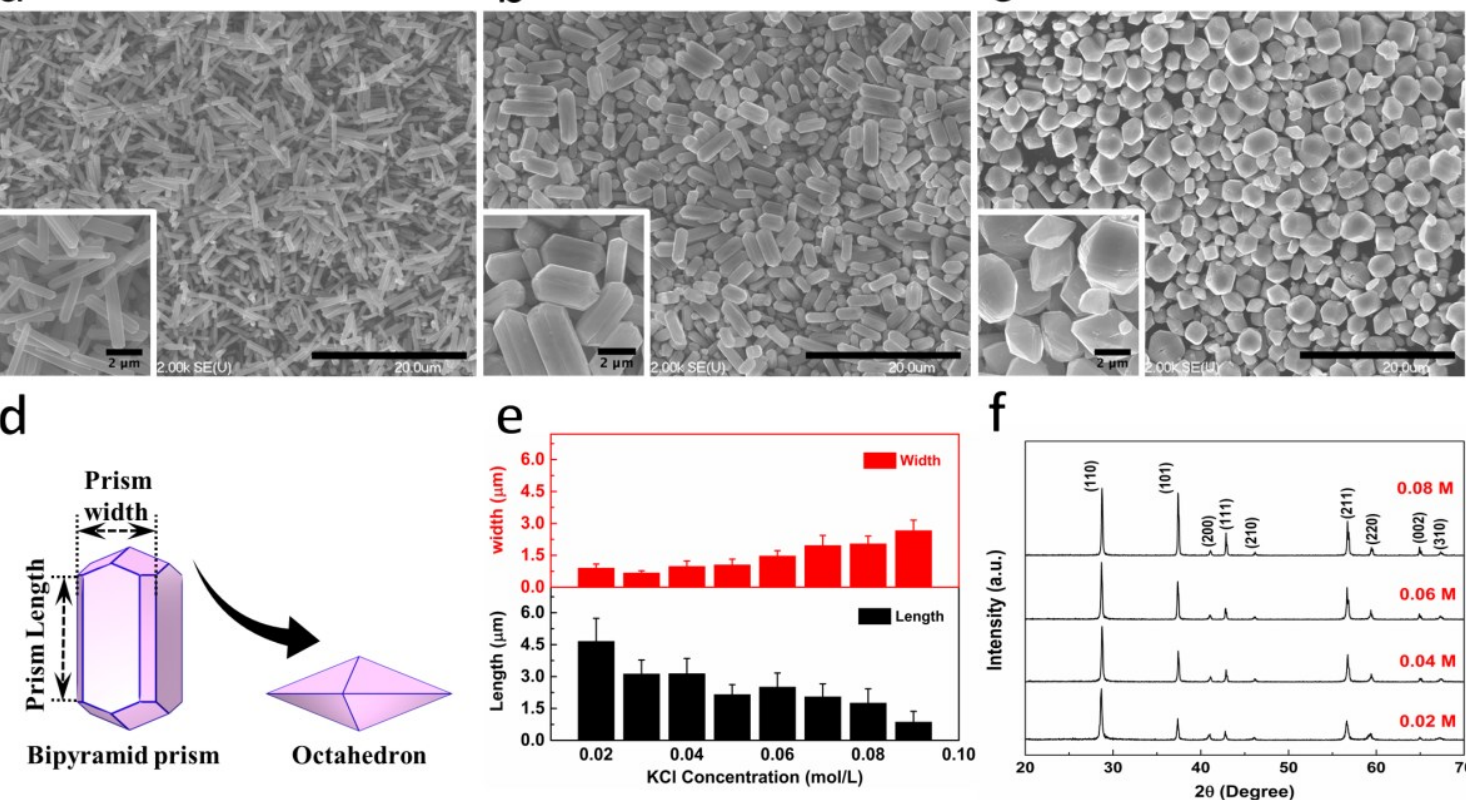

f

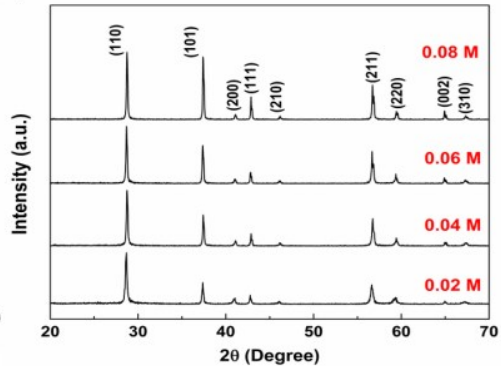

Figure 5-3 FE-SEM images of the $\beta-\mathrm{MnO}_{2}$ microstructures synthesized using precursors with different concentrations of $\mathrm{KCl}$ : (a) $0.03 \mathrm{M}$; (b) $0.06 \mathrm{M}$; (c) $0.09 \mathrm{M}$. Scale bars are 20 $\mu \mathrm{m}$. The inset panels show the corresponding high magnification SEM images of crystals. Scale bars are $2 \mu \mathrm{m}$. (d) Schematic image showing the morphology transition of $\beta-\mathrm{MnO}_{2}$ crystals from bipyramid prism to octahedron. (e) Variation of the length and width of the $\beta-\mathrm{MnO}_{2}$ prism part versus the concentration of $\mathrm{KCl}$. (f) XRD patterns of the synthesized $\beta-\mathrm{MnO}_{2}$ structure obtained under different concentration of $\mathrm{KCl}$.

To verify whether the introduction of extra $\mathrm{Cl}^{-}$anions could affect the morphology of the $\beta-\mathrm{MnO}_{2}$ structure, $0.09 \mathrm{M} \mathrm{KNO}_{3}, \mathrm{NaCl}$, and $\mathrm{NH}_{4} \mathrm{Cl}$ were added individually to replace the $\mathrm{KCl}$ surfactant. When the extra $\mathrm{K}^{+}$ions were kept $\left(\mathrm{KNO}_{3}\right.$, Fig. S5-5a), the morphology of $\beta-\mathrm{MnO}_{2}$ changed to the octahedron-shape. However, when the $\mathrm{K}^{+}$cations were replaced with $\mathrm{Na}^{+}$or $\mathrm{NH}_{4}{ }^{+}$cations, there was no change in the bipyramid prism morphology (Fig. S5-5b, c). The results confirmed that the $\mathrm{K}^{+}$cations were solely responsible for the morphology evolution of $\beta-\mathrm{MnO}_{2}$. 


\subsubsection{Crystal surfaces identification}

To develop the understanding of $\mathrm{K}^{+}$effect on the morphology evolution of $\beta-\mathrm{MnO}_{2}$, detailed analysis of the crystal surfaces was performed by FE-SEM and HRTEM in combined with ultramicrotomy. The growth direction of $\beta-\mathrm{MnO}_{2}$ structures was confirmed to be along the [001] tunnel direction (Fig. 5-4a, e), according to the selected area electron diffraction (SAED) patterns obtained from the [100] zone axis. The identification of the lateral facets was realized based on the diffraction pattern obtained from [001] zone axis. Cross-section sample, therefore, is needed for the analysis of the bipyramid prism morphology (Fig. 5-4b). For the identification of lateral surfaces, it is more accurate to examine the structure along the longitude axis instead of lateral side, because TEM projection images can only provide two-dimensional (2D) information and the orientation of the microrod will greatly affect the diffraction pattern. The lateral surfaces of the bipyramid prism were identified to be the $\{100\}$ facets (Fig. 5-4b, c). The atomic model shows the $\{100\}$ facets of $\beta-\mathrm{MnO}_{2}$ contain a zigzag arrangement of the $\mathrm{Mn}$ atoms (Fig. 54d). Based on the corresponding TEM/SAED pattern (Fig. 5-4e, f, g), the lateral surfaces of the octahedrons were identified to be $\{110\}$ facets. This change of lateral surface from $\{100\}$ to $\{110\}$ was only observed when the concentration of $\mathrm{KCl}$ precursor was higher than $0.08 \mathrm{M}$ and the length of the bipyramid prism was reduced to a much small level. The surface evolution from $\{100\}$ to $\{110\}$ is also consistent with our previous observation, ${ }^{33}$ which is related to the higher surface energy of the $\{100\}$ facets $\left(0.94 \mathrm{~J} / \mathrm{m}^{2}\right)$ compared with $\{110\}$ facets $\left(0.62 \mathrm{~J} / \mathrm{m}^{2}\right)$. Comparable hollow parts were observed in these two morphologies, which is related to the $\mathrm{HCl}$ acid etching effect. With the same amount of 
manganese precursors, the concentration of the generated hydrochloride acid would be the same, which then resulted in a similar size of hollow parts in these structures.

From FE-SEM images in Fig. 5-4, both the bipyramid prism and the octahedron $\beta$ $\mathrm{MnO}_{2}$ showed a four-fold symmetric structure with clear regular crisscross defects. These defects were all generated along the $\{100\}$ planes, as indicated by the yellow dashed lines in Fig. 5-4b and Fig. 5-4f. These defects are possibly generated during the oriented attachment of the nanostructures in order to reduce the overall surface energies. ${ }^{25,34}$ Increasing the concentration of $\mathrm{KCl}$ did not change the oriented attachment, which indicated the extra $\mathrm{K}^{+}$ions change the morphologies of $\beta-\mathrm{MnO}_{2}$ more likely by affecting the growth process.

The pyramid surfaces were further identified by measuring the interplanar angles from FE-SEM images and then compared with theoretical calculation. Previous SAED pattern has confirmed the single-crystalline nature of the individual particles; therefore, by measuring the interplanar angle between the pyramid surface and the prism surface, it is possible to determine the actual crystal plane of the pyramid surface. TEM images were not used here because it is hard to distinguish the orientation of the pyramid surfaces in the 2D projected images. From the FE-SEM images, however, the spatial orientations between two different crystal surfaces can be defined. Two specific interplanar angles were measurable to identify the pyramid surface: one is the interplanar angle between the pyramid surface and $\{110\}$ surface; another is the diagonal slant angle of the pyramid. Representative measurements were performed to identify the pyramid surfaces for both the bipyramid prism and the octahedron structures (Fig. 5-4h, i). Over 100 particles were 
analyzed to ensure the accuracy of these measurements. The interplanar angle between the pyramid surface and the lateral $\{110\}$ surface was found to be $130.9^{\circ} \pm 3.1^{\circ}$. The diagonal slant angle of the pyramid was $116.1^{\circ} \pm 2.6^{\circ}$. Theoretical calculation of different interplanar angles is listed in Table 5-1. Fig. 5-4j shows the theoretical calculation of diagonal slant angles (P1 and P2) for $\{111\}$ and $\{101\}$ pyramids. It is found that the theoretical interplanar angle between $\{111\}$ plane and $\{110\}$ plane is $132.7^{\circ}$, and the diagonal slant angle of $\{111\}$ pyramid is $113.6^{\circ}$. The pyramid surfaces of the $\beta-\mathrm{MnO}_{2}$ bipyramid prism and the octahedron, therefore, are assigned to the $\{111\}$ facets. The domination of $\{111\}$ planes as the pyramid surface is also consistent with previous results. $^{21}$

Table 5-1 Theoretical calculations for different proposed pyramid surfaces

\begin{tabular}{ccccc}
\hline $\begin{array}{c}\text { Proposed } \\
\text { Pyramid } \\
\text { plane }\end{array}$ & $\begin{array}{c}\text { Surface } \\
\text { energy }^{34} \\
\left(\mathbf{J ~ m ~ m}^{-2}\right)\end{array}$ & $\begin{array}{c}\text { Diagonal } \\
\text { slant } \\
\text { angle }\end{array}$ & $\begin{array}{c}\text { Interplanar } \\
\text { angle vs. (110) }\end{array}$ & $\begin{array}{c}\text { Interplanar } \\
\text { angle vs. (100) }\end{array}$ \\
\hline$(101)$ & 1.59 & $130.4^{\circ}$ & $112.8^{\circ}$ & $123.2^{\circ}$ \\
$(111)$ & 1.42 & $113.6^{\circ}$ & $132.7^{\circ}$ & $118.7^{\circ}$ \\
$(311)$ & 1.08 & $54.1^{\circ}$ & $143.6^{\circ}$ & $148.6^{\circ}$ \\
$(321)$ & 1.01 & $54.1^{\circ}$ & $154.5^{\circ}$ & $140.0^{\circ}$ \\
$(211)$ & 1.12 & $74.9^{\circ}$ & $141.5^{\circ}$ & $137.6^{\circ}$ \\
$(201)$ & 1.41 & $94.5^{\circ}$ & $124.2^{\circ}$ & $142.6^{\circ}$ \\
\hline
\end{tabular}




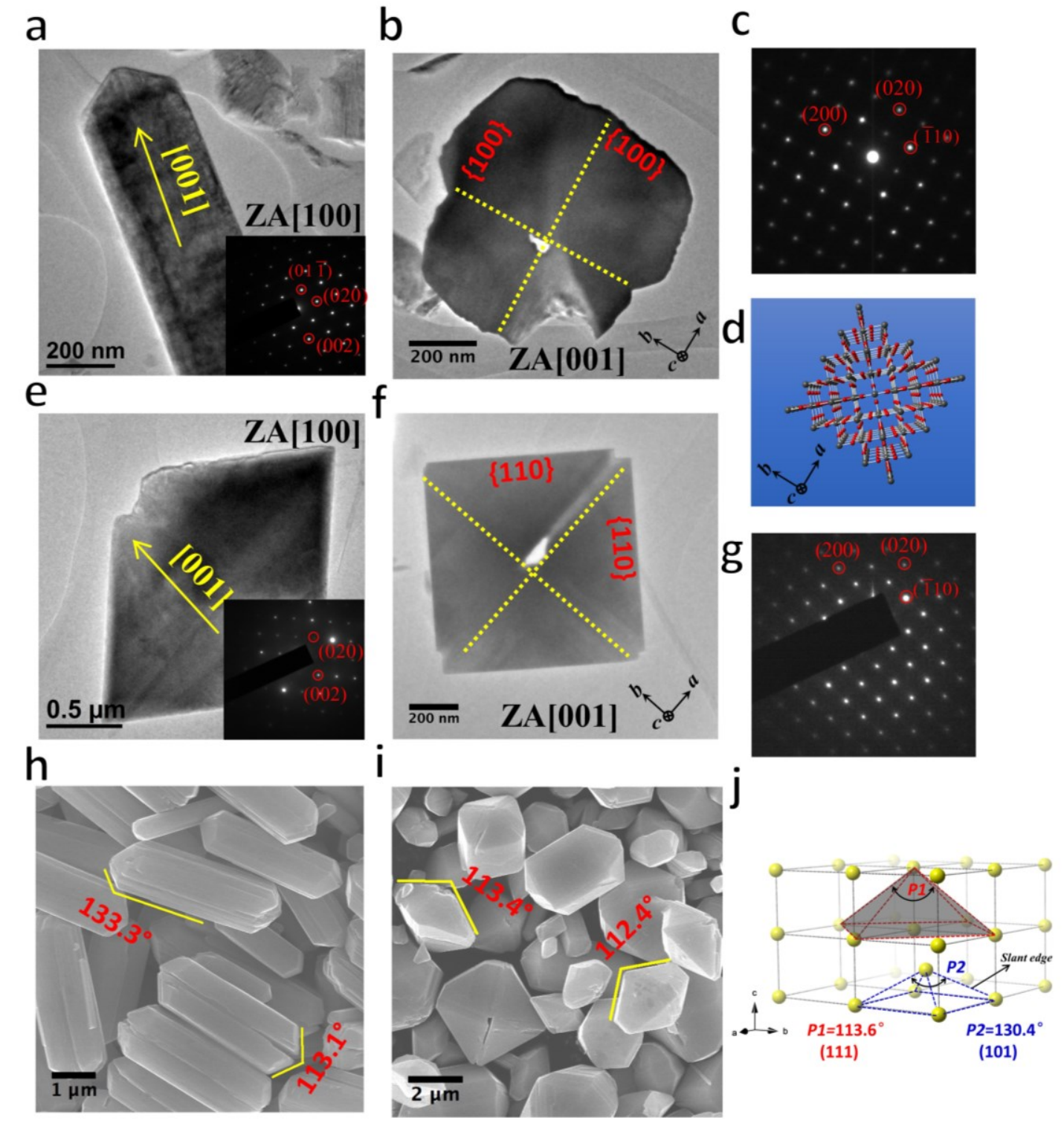

Figure 5-4 (a) TEM image of $\beta-\mathrm{MnO}_{2}$ bipyramid prism viewed along the [100] zone axis; the inset panel shows the corresponding selected area electron diffraction (SAED) pattern. (b) Cross-section TEM image of the $\beta-\mathrm{MnO}_{2}$ bipyramid prism (obtained with $0.03 \mathrm{M} \mathrm{KCl}$ ) viewed along the [001] zone axis, and (c) the corresponding SAED pattern. (d) The atomic model of $\beta-\mathrm{MnO}_{2}$ viewed along the tunnel direction; the red balls represent the oxygen atoms while the grey balls represent the $\mathrm{Mn}$ atoms. (e) $\beta-\mathrm{MnO}_{2}$ octahedron (obtained with $0.09 \mathrm{M} \mathrm{KCl}$ ) viewed along the [100] zone axis; the inset panel gives the SAED pattern. (f) $\beta-\mathrm{MnO}_{2}$ octahedron viewed along the [001] zone axis, and $(\mathrm{g})$ the corresponding SAED 
pattern. (h) Representative angle measurements to identify the pyramid surfaces in the $\beta$ $\mathrm{MnO}_{2}$ bipyramid prism structures. (i) Representative angle measurements in the $\beta-\mathrm{MnO}_{2}$ octahedra. (j) Schematic atom model illustrating the diagonal slant angles of the pyramids consisted of $\{111\}$ or $\{101\}$ planes. Yellow atoms indicate the location of Mn atoms. Body-centered Mn atom was only shown in the unit cell (P2) for better vision.

Previous modeling works predicted the prominence of the $\{101\}$ planes as the pyramid surfaces in the equilibrium $\beta-\mathrm{MnO}_{2}$ crystals. ${ }^{35-37}$ However, based on our theoretical calculation, the diagonal angle of $\{101\}$ pyramid was $130.4^{\circ}$, which was far larger than the measured $116.1^{\circ}$. The interplanar angles between $\{101\}$ and $\{110\}$ were calculated to be $112.8^{\circ}$, which were also deviated too much from the measured $130.9^{\circ}$. On the other hand, the surface energies of $\{101\}$ and $\{111\}$ planes were predicted to be $1.59 \mathrm{~J}$ $\mathrm{m}^{-2}$ and $1.42 \mathrm{~J} \mathrm{~m}{ }^{-2}$ by PBE$+\mathrm{U}$ method. ${ }^{34}$ Because of the lower surface energy, the $\{111\}$ planes would be more favorable than the $\{101\}$ planes. In summary, increasing the concentration of $\mathrm{K}^{+}$ions increased the occupancy of the bipyramid $\{111\}$ surfaces and reduced the occupancy of the lateral $\{100\}$ surfaces in the $\beta-\mathrm{MnO}_{2}$ microstructures. This conclusion is also consistent with XRD patterns, where the integrated peak intensity ratio of $\mathrm{I}_{(111)} / \mathrm{I}_{(110)}$ increased with the introduction of more $\mathrm{K}^{+}$cations.

\subsubsection{The role of $\mathrm{K}^{+}$cations during the morphology evolution of $\beta-\mathrm{MnO}_{2}$}

The formation of the $\beta-\mathrm{MnO}_{2}$ microstructures followed oriented attachment of $\mathrm{K}^{+}$ stabilized $\alpha-\mathrm{MnO}_{2}$ nanowires and subsequent phase transition to $\beta-\mathrm{MnO}_{2}$. The $\mathrm{K}^{+}$cations affect the morphology of $\beta-\mathrm{MnO}_{2}$ through two aspects. On one hand, the cations affected the formation of $\alpha-\mathrm{K}_{0.5} \mathrm{Mn}_{4} \mathrm{O}_{8}$ phase. Before oriented attachment, higher concentration of $\mathrm{K}^{+}$cations facilitated the formation of $\alpha-\mathrm{K}_{0.5} \mathrm{Mn}_{4} \mathrm{O}_{8}$ intermediate phase. Under the same 
temperature and pressure conditions but a higher concentration of $\mathrm{K}^{+}$cations, a relatively larger amount of $\alpha-\mathrm{K}_{0.5} \mathrm{Mn}_{4} \mathrm{O}_{8}$ nanowires would be expected and the phase transition from $\alpha-\mathrm{K}_{0.5} \mathrm{Mn}_{4} \mathrm{O}_{8}$ to $\beta-\mathrm{MnO}_{2}$ will be postponed. This offers more time for oriented attachment of $\alpha-\mathrm{K}_{0.5} \mathrm{Mn}_{4} \mathrm{O}_{8}$ nanowires. Thus, an increased width of the $\beta-\mathrm{MnO}_{2}$ was observed under higher concentration of $\mathrm{K}^{+}$cations. On the other hand, $\mathrm{K}^{+}$cations affect the phase transition

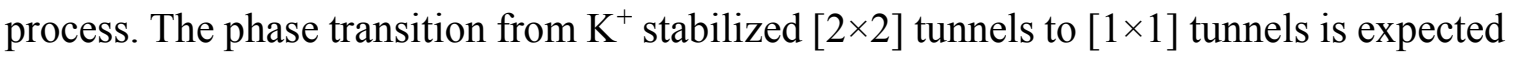
to follow two steps. The first step is the extraction of the $\mathrm{K}^{+}$ions out of the [2×2] tunnels; the second step is the tunnel transition from [2×2] to [1×1] tunnels. As shown in Fig. 5-5, the higher concentration of $\mathrm{K}^{+}$ions in the solution $(0.02 \sim 0.09 \mathrm{M})$ would impede the extraction of $\mathrm{K}^{+}$ions from the [2×2] tunnels because of the relatively lower $\mathrm{K}^{+}$ concentration in the $\alpha-\mathrm{K}_{0.5} \mathrm{Mn}_{4} \mathrm{O}_{8}$ solid $(0.0148 \mathrm{M})$ compared with that in the solution. The concentration of $\mathrm{K}^{+}$cations in the solution will determine the length of [2×2] tunnels that

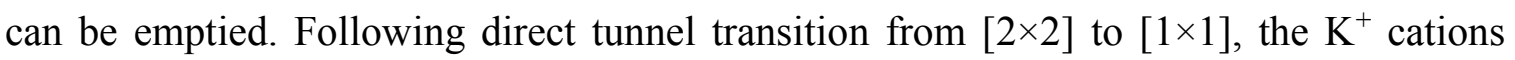
controlled the length of obtainable $\beta-\mathrm{MnO}_{2}[1 \times 1]$ tunnels. The high concentration of $\mathrm{K}^{+}$ cations, therefore, reduced the length of $\beta-\mathrm{MnO}_{2}$ along the tunnel direction.

It should be mentioned that the existence of $\alpha-\mathrm{K}_{0.5} \mathrm{Mn}_{4} \mathrm{O}_{8}$ intermediate phase is necessary for the $\mathrm{K}^{+}$ions to affect the morphology of $\beta-\mathrm{MnO}_{2}$. Previous study has demonstrated that the molar ratio between $\mathrm{KMnO}_{4}$ and $\mathrm{MnSO}_{4} \cdot \mathrm{H}_{2} \mathrm{O}$ determines the phase of obtained $\mathrm{MnO}_{2}$, and $\beta-\mathrm{MnO}_{2}$ is more favorable when the molar ratio is lower than 2:3. ${ }^{16}$ With a molar ratio of 2:3, $\alpha-\mathrm{K}_{0.5} \mathrm{Mn}_{4} \mathrm{O}_{8}$ can exist as the intermediate phase based on our observations. With low concentration of $\mathrm{KCl}(0.02 \mathrm{M})$, both $\alpha-\mathrm{K}_{0.5} \mathrm{Mn}_{4} \mathrm{O}_{8}$ and $\gamma-\mathrm{MnO}_{2}$ with $[1 \times 2]$ tunnels acted as the intermediate phase (Fig. S5-6). When the molar ratio was further 
reduced to $1: 3$ or $1: 6, \alpha-\mathrm{K}_{0.5} \mathrm{Mn}_{4} \mathrm{O}_{8}$ was not expected; adding extra $\mathrm{KCl}$ did not change the morphology of the $\beta-\mathrm{MnO}_{2}$ microrod, although we tried to compensate the same amount of $\mathrm{K}^{+}$cations inside the autoclave (Fig. S5-7). This is because the $\mathrm{K}^{+}$ion does not exist in the [1×2] tunnels of $\gamma-\mathrm{MnO}_{2}$, therefore extra $\mathrm{K}^{+}$ions cannot affect the phase transition from $[1 \times 2]$ to $[1 \times 1]$. On the other hand, with high concentration of $\mathrm{KCl}(0.09 \mathrm{M})$, the phase transition from $\alpha-\mathrm{K}_{0.5} \mathrm{Mn}_{4} \mathrm{O}_{8}$ to $\beta-\mathrm{MnO}_{2}$ was postponed, due to the facilitated formation of alpha phases at high $\mathrm{K}^{+}$conditions (Fig. S5-8). Adding more than $0.09 \mathrm{M} \mathrm{K}^{+}$cations in the system while keeping the same molar ratio of 2:3, a mixture of $\beta-\mathrm{MnO}_{2}$ and $\alpha-\mathrm{K}_{0.5} \mathrm{Mn}_{4} \mathrm{O}_{8}$ will be expected based on previous study, ${ }^{19}$ and the size of $\beta-\mathrm{MnO}_{2}$ will get larger.

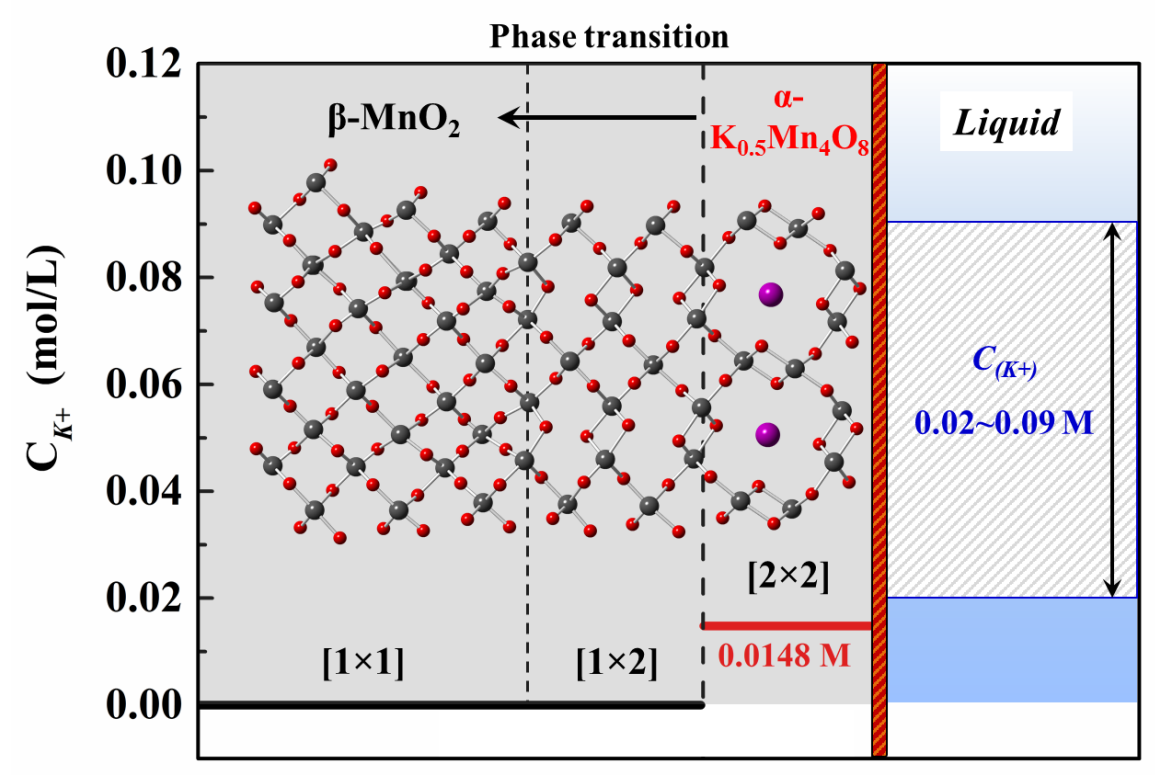

Figure 5-5 Schematic graph illustrates the effect of $\mathrm{K}^{+}$cations on the phase transition from $\alpha-\mathrm{K}_{0.5} \mathrm{Mn}_{4} \mathrm{O}_{8}$ to $\beta-\mathrm{MnO}_{2}$. The red balls represent the oxygen atoms; the grey balls represent the $\mathrm{Mn}$ atoms; the purple balls represent the $\mathrm{K}^{+}$cations.

To further demonstrate the growth mechanism and phase transition process, DFT simulations were conducted on pure $\mathrm{K}, \mathrm{Mn}$, and $\mathrm{O}$ systems, as well as different phases of 
$\mathrm{K}_{\mathrm{x}} \mathrm{Mn}_{8} \mathrm{O}_{16}$ to determine their relative stabilities. For the $\mathrm{K}_{x} \mathrm{Mn}_{8} \mathrm{O}_{16}$ systems, $\mathrm{K}^{+}$ions were inserted into 2a, 2b, 4c, 4d, 4e, 8f, and 8g Wyckoff sites. Fig. 6 shows the energies of formation for the $\alpha-\mathrm{MnO}_{2}, \beta-\mathrm{MnO}_{2}$, ortho- $\mathrm{MnO}_{2}$, and $\mathrm{K}_{x} \mathrm{Mn}_{8} \mathrm{O}_{16}$ systems for $x=0$ to 4 . It is evident that the $\mathrm{K}^{+}$ions inserted into the $\alpha-\mathrm{MnO}_{2}$ system are most likely to be found in the $2 \mathrm{~b}$ or $4 \mathrm{e}$ sites for the first $\mathrm{K}^{+}$ion $(x=1) ; 2 \mathrm{a}, 2 \mathrm{~b}$, or $4 \mathrm{c}+4 \mathrm{e}$ sites for the second $\mathrm{K}^{+}$ion $(x$ $=2$ ); and $4 \mathrm{c}$ sites for the third and fourth $\mathrm{K}^{+}$ions $(x=3$ and 4$)$. Thus, the initial insertion points are in the $[2 \times 2]$ tunnels, followed by insertion into the $[1 \times 1]$ tunnels once the $[2 \times 2]$ tunnels are saturated. The $\alpha-\mathrm{MnO}_{2}$ with one $\mathrm{K}^{+}$ion $\left(\alpha-\mathrm{K}_{0.5} \mathrm{Mn}_{4} \mathrm{O}_{8}\right)$ shows lower formation energy than the $\beta-\mathrm{MnO}_{2}$. This supports our observation that $\alpha-\mathrm{MnO}_{2}$ nanowires were formed before transferring to $\beta-\mathrm{MnO}_{2}$.

The phase transition from $\alpha-\mathrm{K}_{0.5} \mathrm{Mn}_{4} \mathrm{O}_{8}$ to $\beta-\mathrm{MnO}_{2}$ was driven by the high temperature, high pressure, and acid solution $\left(\mathrm{H}^{+}\right.$effect). Once $\mathrm{K}^{+}$ions are driven out, the beta phase $\mathrm{MnO}_{2}$ is the most stable structure, followed by the ortho [1×2] and alpha $[2 \times 2]$ phases, which have nearly identical formation energies. This supports our observation that the $[2 \times 2]$ tunnels transform to $[1 \times 1]$ tunnels with the $[1 \times 2]$ tunnels (ortho phase) as an intermediate phase after $\mathrm{K}^{+}$ions are driven out.

With low concentration of $\mathrm{KCl}$ surfactant, gamma phase $\mathrm{MnO}_{2}$ with ortho [1×2] tunnels were also detected as the intermediate phase before phase transition to beta $\mathrm{MnO}_{2}$. This phase transition from gamma to beta $\mathrm{MnO}_{2}$ is also believed to follow the oriented attachment with alpha $\mathrm{MnO}_{2}$ and then the direct phase transition to beta $\mathrm{MnO}_{2}$, because of the relatively higher thermostability of the beta phase. $\mathrm{K}^{+}$cations are not needed for the formation of [1×2] tunnels of $\mathrm{MnO}_{2}$, further confirmed by our EELS analysis (as shown in 
Fig. 5-2e). The concentration of $\mathrm{KCl}$ surfactant, therefore, will not affect the phase transition of gamma phase to beta phase $\mathrm{MnO}_{2}$. The role of $\mathrm{K}^{+}$cations effect on the phase transition would be only related to the alpha phase $\mathrm{MnO}_{2}$.

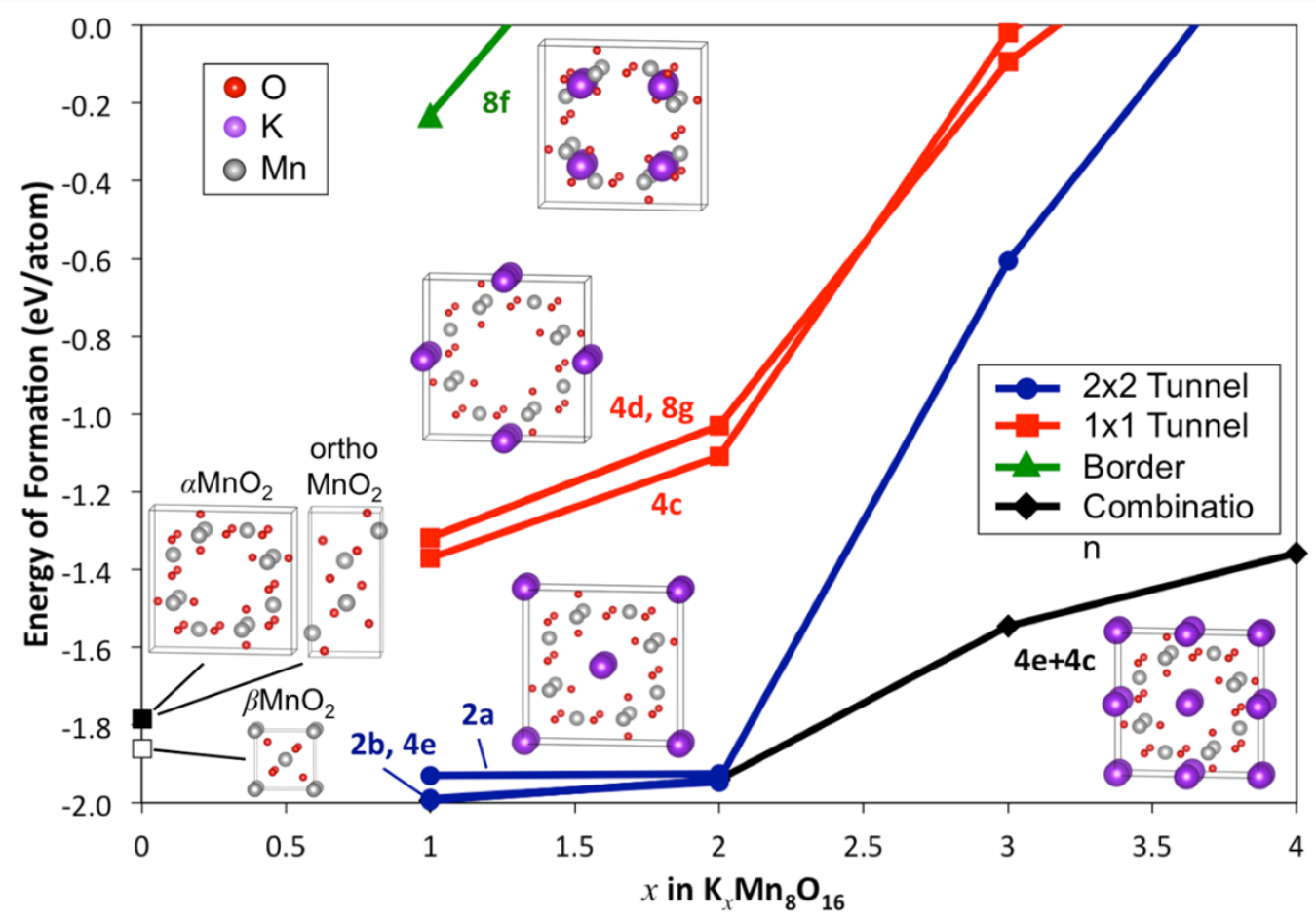

Figure 5-6 Energies of formation for different $\mathrm{MnO}_{2}$ phases as a function of inserted $\mathrm{K}^{+}$ ions.

\subsubsection{Electrochemical performance as the cathode materials for Li-ion batteries}

The tunable growth of $\beta-\mathrm{MnO}_{2}$ with different occupancy of $\{111\}$ and $\{100\}$ facets can find wide applications in energy storage devices, such as lithium or sodium-ion batteries, cathode catalyst for metal-air batteries, supercapacitors, etc. In this work, $\beta-\mathrm{MnO}_{2}$ microstructures obtained under $0.04 \mathrm{M}$ and $0.08 \mathrm{M} \mathrm{KCl}$ (highly exposed $\{111\}$ facets) surfactant were chosen to study the effect of crystal facets on the rate performance of $\beta$ $\mathrm{MnO}_{2}$ as lithium-ion battery cathode. The surface areas of the two microstructures were 
determined based on the nitrogen adsorption isotherms. According to the International Union of Pure and Applied Chemistry (IUPAC) classification, the two structures showed the same Type-III adsorption isotherms, indicating nonporous material with weak adsorbate-adsorbent interactions (Fig. S5-9). ${ }^{38}$ The Brunauer-Emmett-Teller (BET) surface areas of the two structures were calculated to be $4.57 \mathrm{~m}^{2} / \mathrm{g}$ and $6.48 \mathrm{~m}^{2} / \mathrm{g}$, respectively.

The representative charge-discharge curves for $\beta-\mathrm{MnO}_{2}$ bipyramid prism $(0.04 \mathrm{M})$ and octahedron $(0.08 \mathrm{M})$ were shown in Fig. S5-10a. The coin cell was cycled with a high discharge cut-off potential at $2.0 \mathrm{~V}\left(\mathrm{vs} . \mathrm{Li} / \mathrm{Li}^{+}\right)$to avoid rupture of the tunnel structures, because of the phase transition from $\mathrm{MnO}_{2}$ to $\mathrm{Mn}$ at $\sim 1.7 \mathrm{~V} .{ }^{39}$ Both charge-discharge curves showed the same plateaus at around $2.8 \mathrm{~V}$ and $3.0 \mathrm{~V}$, indicating a reversible lithium ion intercalation and deintercalation in the $\beta-\mathrm{MnO}_{2}[1 \times 1]$ tunnels. The small plateau at 4.0 $\mathrm{V}$ corresponds to the phase transition from rutile $\mathrm{MnO}_{2}$ to spinel $\mathrm{LiMn}_{2} \mathrm{O}_{4}{ }^{40-42}$ A relatively longer intercalation plateau was observed in the bipyramid prism structure $(0.04 \mathrm{M})$ due to its longer prism length from charge storage. With the similar surface areas, the bipyramid prism morphology is expected to provide larger volume for charge storage than the octahedron.

The rate performance of the $\beta-\mathrm{MnO}_{2}$ structures was studied with the same potential window $\left(2.0 \sim 4.2 \mathrm{~V}\right.$ vs. $\left.\mathrm{Li} / \mathrm{Li}^{+}\right)$as shown in Fig. $5-7 \mathrm{a}$. The specific capacity obtained after 7 cycles at $20 \mathrm{~mA} / \mathrm{g}$ was used to normalize all the other capacities. Around $70 \%$ capacity was maintained for the octahedron structure $(0.08 \mathrm{M})$ with highly exposed $\{111\}$ facets when the charge-discharge current increased from $20 \mathrm{~mA} / \mathrm{g}$ to $200 \mathrm{~mA} / \mathrm{g}$. Only $50 \%$ 
capacity was maintained for the bipyramid prism structure $(0.04 \mathrm{M})$. The better rate performance of the octahedron structure is due to the highly exposed $\{111\}$ facets, which offers more tunnel openings for lithium ion transport. The capacity of the beta $\mathrm{MnO}_{2}$ structures increased upon cycling, which can be related to the activation of the inner parts of the $\mathrm{MnO}_{2}$ crystals. The lithium ion diffusion in the two $\beta-\mathrm{MnO}_{2}$ structures was also evaluated using electrochemical impedance spectroscopy (EIS). The Nyquist plots were shown in Fig. 5-7b. The fitted impedance parameters are listed in Table S5-1. The charge transfer resistance (R3) and solution resistance (R1) were nearly identical for the two systems, indicating a consistent fabrication of the electrodes and the assembling of the coin cells. A clearly higher lithium ion diffusion resistance (W1) was found $\left(239.8 \Omega \cdot \mathrm{s}^{-1 / 2} \mathrm{vs}\right.$. $\left.177.9 \Omega \cdot \mathrm{s}^{-1 / 2}\right)$ inside the bipyramid prism structure $(0.04 \mathrm{M})$. The Warburg coefficients $(\sigma)$ for the two samples were also obtained (Fig. S5-10b) through linear fitting of the impedance versus the $-1 / 2$ power of the angular frequency $\left(\omega^{-1 / 2}\right) \cdot{ }^{27}$ Lower Warburg coefficient was also observed for the $\beta-\mathrm{MnO}_{2}$ octahedrons $(0.08 \mathrm{M})$, indicating better lithium ion mobility in this structure (Table S5-1). After cycling, the $\beta-\mathrm{MnO}_{2}$ microstructures still maintained their bipyramid prism and octahedron morphology (Fig. $5-7 \mathrm{c}, \mathrm{d})$. These results indicated the $\beta-\mathrm{MnO}_{2}$ octahedrons with highly exposed $\{111\}$ facets offer higher lithium ion diffusion mobility and better rate performance.

Considering that most of the crystal structure is still occupied with $[1 \times 1]$ tunnels and similar defects are observed both in the bipyramid prism and octahedron structures, the effect of crystal defects on the rate performance of the two $\beta-\mathrm{MnO}_{2}$ structures was ignored in this work. The size of the $\beta-\mathrm{MnO}_{2}$ structures obtained here are close to bulk 
materials, which limited its electrochemical performance as the cathode for lithium-ion batteries. If the oriented attachment of $\alpha-\mathrm{K}_{0.5} \mathrm{Mn}_{4} \mathrm{O}_{8}$ nanowires can be limited by proper surfactants while keeping the effect of $\mathrm{K}^{+}$cations, it is possible to further reduce the size of the $\beta-\mathrm{MnO}_{2}$ structures and improve their electrochemical performance. Although more effort is still needed, the controlled growth of $\beta-\mathrm{MnO}_{2}$ crystals provided here not only reveals a new route to improve the rate performance of this crystal but also brings new aspects for the design of this material towards its application in supercapacitors, metal-air batteries, and fuel cells.
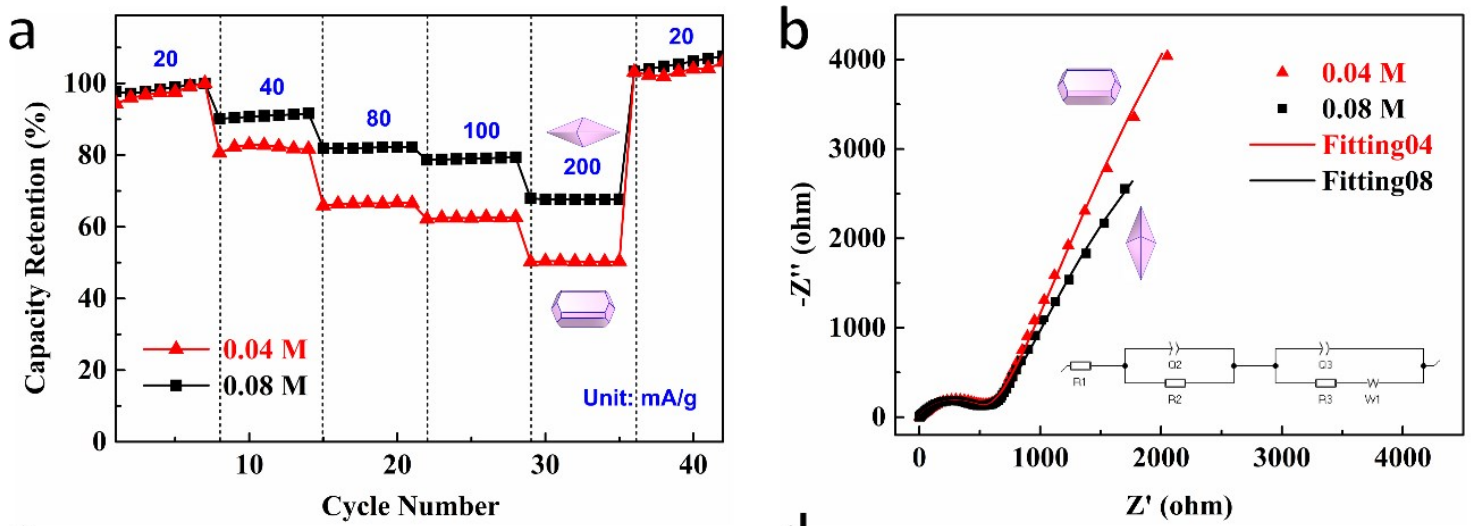

C

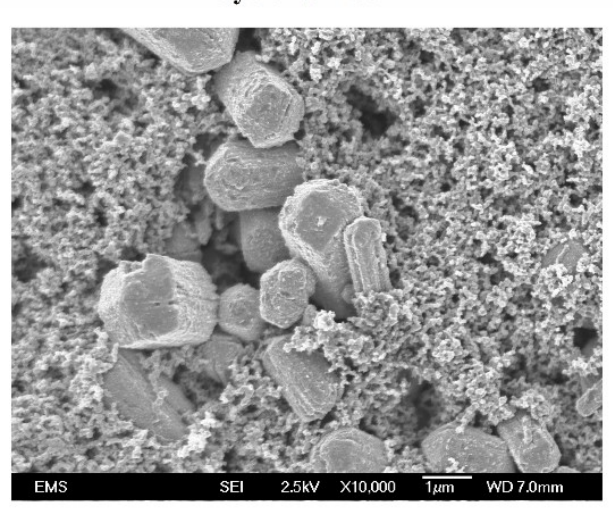

d

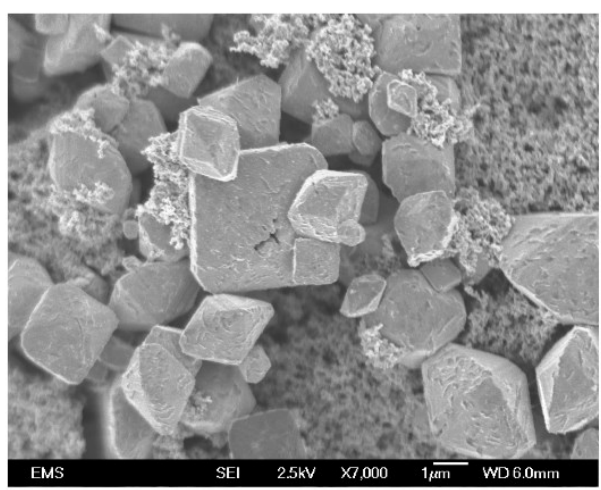

Figure 5-7 (a) Rate performance of $\beta-\mathrm{MnO}_{2}$ bipyramid prism $(0.04 \mathrm{M})$ and octahedron $(0.08 \mathrm{M})$ at various current densities from $20 \mathrm{~mA} \cdot \mathrm{g}^{-1}$ to $200 \mathrm{~mA} \cdot \mathrm{g}^{-1}$. (b) Nyquist plots of the lithium half-cells assembled with the two structures as the working electrode; the inset 
panel gives the corresponding equivalent circuit for the fitting of the experimental data. R1 represents the electrolyte resistance; $\mathrm{R} 2$ represents the resistance of charge transfer through the solid electrolyte interfaces (SEI); Q2 represents the capacitance of the SEI layer; R3 represents the charge transfer resistance at the electrode interfaces; Q3 represents the capacitance of electrical double layers; W1 represents the resistance of lithium ion diffusion inside the electrode. The FE-SEM images showing the $\beta-\mathrm{MnO}_{2}$ crystals maintained their (c) bipyramid prism and (d) octahedron morphologies after cycling.

\subsection{Conclusions}

In summary, synthesis of $\beta-\mathrm{MnO}_{2}$ crystal structures with different occupancy of $\{110\}$ and $\{111\}$ facets were realized through the effect of $\mathrm{K}^{+}$cations. Ex-situ study showed that the $\beta-\mathrm{MnO}_{2}$ microstructure was formed through the oriented attachment of $\alpha-\mathrm{K}_{0.5} \mathrm{Mn}_{4} \mathrm{O}_{8}$ nanowires and a subsequent direct phase transition. The transition from $\alpha-\mathrm{K}_{0.5} \mathrm{Mn}_{4} \mathrm{O}_{8}[2 \times 2]$ tunnels to $\beta-\mathrm{MnO}_{2}[1 \times 1]$ tunnels was clarified as a direct solid-state transition process, with $[1 \times 2]$ tunnels as a possible intermediate phase. Further cations effect study showed that the high concentration of $\mathrm{K}^{+}$cations reduced the prism length of the $\beta-\mathrm{MnO}_{2}$ crystals through affecting the formation of $\alpha-\mathrm{K}_{0.5} \mathrm{Mn}_{4} \mathrm{O}_{8}$ nanowires and the tunnel transition process. DFT simulation further demonstrate the phase transition from $\alpha-\mathrm{MnO}_{2}$ without $\mathrm{K}^{+}$stabilizing ions and gamma $\mathrm{MnO}_{2}$ to beta $\mathrm{MnO}_{2}$ based on their different formation energies. As the cathode material for lithium-ion batteries, the $\beta-\mathrm{MnO}_{2}$ octahedrons with highly exposed $\{111\}$ facets gave better rate performance than the bipyramid prism structure. Our findings revealed a unique way to tune the morphology of $\beta-\mathrm{MnO}_{2}$ crystals. The structures obtained here can also be used to evaluate the effect of crystal facets on the electrochemical performance of this metal oxide as supercapacitors and catalysts for metal-air batteries and fuel cells. 


\subsection{Methods}

\subsubsection{Materials Synthesis}

$\beta-\mathrm{MnO}_{2}$ crystals were synthesized following a hydrothermal method. First, $0.08 \mathrm{M} \mathrm{KMnO}_{4}$ and $0.12 \mathrm{M} \mathrm{MnCl}_{2} \cdot 4 \mathrm{H}_{2} \mathrm{O}$ were dissolved in $80 \mathrm{~mL}$ DI water. Then, different amount of $\mathrm{K}^{+}$ ions were introduced by adding $\mathrm{KCl}$ into the solution under constant stirring. The concentration of $\mathrm{KCl}$ varies from $0.02 \mathrm{M}$ to $0.09 \mathrm{M}$. The mixture was stirred continuously for $30 \mathrm{~min}$ before transferring to a $100 \mathrm{~mL}$ Teflon-lined stainless-steel autoclave. The autoclave was sealed and heated at $180{ }^{\circ} \mathrm{C}$ for $12 \mathrm{~h}$. The obtained $\mathrm{MnO}_{2}$ crystals were collected by centrifugation and then washed with DI water and ethyl alcohol for three times, respectively. The final products were dried in air at $60^{\circ} \mathrm{C}$ for $12 \mathrm{~h}$. To distinguish the effect of $\mathrm{K}^{+}$ions on the morphology of the $\beta-\mathrm{MnO}_{2}$ from other ions, $0.09 \mathrm{M} \mathrm{NH}_{4} \mathrm{Cl}$, $\mathrm{KNO}_{3}$, and $\mathrm{NaCl}$ were used to replace the $0.09 \mathrm{M} \mathrm{KCl}$ individually.

\subsubsection{Cross-section sample preparation}

Cross-section samples were prepared based on the ultramicrotomy method. $\beta-\mathrm{MnO}_{2}$ crystals were first mixed with cold mounting epoxy resin (EPOFIX, Electron Microscopy Sciences) and hardener (EPOFIX, Triethylenetetramine, Electron Microscopy Sciences). Then the mixture was sonicated for 10 min to reduce bubble formation. After the mixture was fully solidified, it was mounted on a Leica Ultracut UCT ultramicrotome. The mixture was sliced with a diamond knife with a feeding step size of $500 \mathrm{~nm}$. Sliced samples were directly transferred onto TEM grids for further characterization. 


\subsubsection{Materials Characterization}

The phases of the synthesized $\mathrm{MnO}_{2}$ structures were identified by X-ray powder diffraction (XRD) using a Scintag XDS2000 powder diffractometer. The diffraction peaks were collected with a scan step of $0.02^{\circ} \mathrm{min}^{-1}$ by $\mathrm{Cu} \mathrm{K} \alpha$ radiation. The morphology of the $\mathrm{MnO}_{2}$ structures was examined by a Hitachi S-4700 field emission scanning electron microscope (FE-SEM). The surface area was determined by $\mathrm{N}_{2}$ adsorption isotherm using a Quadrasorb SI analyzer at $77 \mathrm{~K}$. The cross-section structure was studied by a $300 \mathrm{kV}$ high-resolution transmission electron microscopy (HRTEM, JEOL 3010) and a $200 \mathrm{kV}$ cold-field emission aberration-corrected JEOL JEM-ARM200CF scanning transmission electron microscopy (STEM) equipped with energy-dispersive x-ray spectroscopy (EDS) and Gatan Electron Energy Loss Spectroscopy (EELS). The EELS spectra were acquired with a $45 \mathrm{mrad}$ collection angles.

\subsubsection{DFT modeling}

All simulations were performed using the Vienna Ab Initio Simulation Package (VASP), version 5.2.12. ${ }^{43-45}$ The Perdew-Burke-Ernzerhof exchange-correlation functional was used ${ }^{46,47}$ with an optimized cut-off energy of $560 \mathrm{eV}$. The DFT+U method was used for these simulations, as implemented in VASP using the approach of Dudarev et al.. ${ }^{48}$ For the transition metal Mn, a U-J value of 5.2 was assumed, based on the results of previous work. ${ }^{49}$ For each of the simulated systems, optimized $\Gamma$-centered Monkhorst-Pack ${ }^{50} \mathrm{~K}$ point grids were established and are provided in Table S2. Relaxation simulations were performed for ionic positions, unit cell shape, and unit cell size. Each of the simulated systems is shown in Table S2 with the corresponding space group. For the K atoms, the 3s, 
$3 p$, and $4 s$ states were treated as valence states. For Mn, the $3 p$, 3d, and $4 s$ states were treated as valence states. For $\mathrm{O}$, the $2 \mathrm{~s}$ and $2 \mathrm{p}$ states were treated as valence states. The energy of formation (per atom) was calculated using

$$
E_{f}=E-n_{K} E_{K}-n_{M n} E_{M n}-n_{O} E_{O}
$$

where $E$ (in $\mathrm{eV} /$ atom) is the total electronic energy of the simulated system (per atom); $E_{K}$, $E_{M n}$, and $E_{O}$ are the energy/atom for the pure K, Mn, and $\mathrm{O}$ systems, respectively; and $n_{K}$, $n_{M n}$, and $n_{O}$ are the mole fractions of $\mathrm{K}, \mathrm{Mn}$, and $\mathrm{O}$ in the simulated system, respectively.

\subsubsection{Electrochemical tests}

All the battery performance tests were performed on a Bio-Logic VMP3 electrochemical workstation. The working electrodes were prepared by mixing synthesized $\beta-\mathrm{MnO}_{2}$, super $\mathrm{P}$, and polyvinylidene difluoride (PVDF) with a weight ratio of 7:2:1. The mixture was mixed with N-methylpyrrolidone (NMP) solvent under constant stirring to form slurry and then coated on an aluminum foil. The thickness of electrode was controlled at $90 \mu \mathrm{m}$. The electrode was then dried at $70{ }^{\circ} \mathrm{C}$ in a vacuum oven for $12 \mathrm{~h}$. Standard CR2032 coin cells were assembled in an Ar-filled glovebox (MBraun) with oxygen and water level below 0.5 ppm. The commercial lithium-ion battery electrolyte (1.0 $\mathrm{M} \mathrm{LiPF}_{6}$ in ethylene carbonate $(\mathrm{EC})$ and diethyl carbonate $(\mathrm{DEC}), \mathrm{EC} / \mathrm{DEC}=50 / 50(\mathrm{v} / \mathrm{v}))$ was purchased from SigmaAldrich and used as received. The coin cells were assembled with lithium foil as the anode and glass fiber as the separator. EIS was performed with a $10 \mathrm{mV}$ AC amplitude and a frequency range from $10 \mathrm{mHz}$ to $100 \mathrm{kHz}$. The galvanostatic charge-discharge cycling was performed with a potential range from $2.0 \mathrm{~V}$ to $4.2 \mathrm{~V}$ vs. $\mathrm{Li} / \mathrm{Li}^{+}$. All the electrochemical tests were performed at room temperature. 


\subsection{References}

1 Zhang, K. et al. Nanostructured Mn-based oxides for electrochemical energy storage and conversion. Chem. Soc. Rev. 44, 699-728 (2015).

2 Chen, J. \& Cheng, F. Combination of lightweight elements and nanostructured materials for batteries. Acc. Chem. Res. 42, 713-723 (2009).

3 Cheng, F. et al. Facile controlled synthesis of $\mathrm{MnO}_{2}$ nanostructures of novel shapes and their application in batteries. Inorg. Chem. 45, 2038-2044 (2006).

4 Ghodbane, O., Pascal, J.-L. \& Favier, F. Microstructural effects on charge-storage properties in $\mathrm{MnO}_{2}$-based electrochemical supercapacitors. ACS Appl. Mater. Interfaces 1, 1130-1139 (2009).

5 Devaraj, S. \& Munichandraiah, N. Effect of crystallographic structure of $\mathrm{MnO}_{2}$ on its electrochemical capacitance properties. J. Phys. Chem. C 112, 4406-4417 (2008).

6 Truong, T. T., Liu, Y., Ren, Y., Trahey, L. \& Sun, Y. Morphological and crystalline evolution of nanostructured $\mathrm{MnO}_{2}$ and its application in lithium-air batteries. ACS Nano 6, 8067-8077 (2012).

7 Xiao, W., Wang, D. \& Lou, X. W. Shape-controlled synthesis of $\mathrm{MnO}_{2}$ nanostructures with enhanced electrocatalytic activity for oxygen reduction. $J$. Phys. Chem. C 114, 1694-1700 (2009).

8 Xiao, W., Xia, H., Fuh, J. Y. \& Lu, L. Growth of single-crystal $\alpha-\mathrm{MnO}_{2}$ nanotubes prepared by a hydrothermal route and their electrochemical properties. J. Power Sources 193, 935-938 (2009).

$9 \mathrm{Li}, \mathrm{W}$. et al. Performance modulation of $\alpha-\mathrm{MnO}_{2}$ nanowires by crystal facet engineering. Sci. Rep. 5 (2015).

10 Kakizaki, H. et al. Evidence that crystal facet orientation dictates oxygen evolution intermediates on rutile manganese oxide. Adv. Funct. Mater. (2018).

11 Kitchaev, D. A., Dacek, S. T., Sun, W. \& Ceder, G. Thermodynamics of Phase Selection in $\mathrm{MnO} 2$ Framework Structures through Alkali Intercalation and Hydration. J. Am. Chem. Soc 139, 2672-2681 (2017).

12 Jiao, F. \& Bruce, P. G. Mesoporous crystalline $\beta-\mathrm{MnO}_{2}$-a reversible positive electrode for rechargeable lithium batteries. Adv. Mater. 19, 657-660 (2007). 
13 Zhan, D., Zhang, Q., Hu, X. \& Peng, T. Single-crystal $\beta-\mathrm{MnO}_{2}$ hollow bipyramids: synthesis and application in lithium ion batteries. RSC Adv. 3, 51415147 (2013).

14 Zang, J. \& Li, X. In situ synthesis of ultrafine $\beta-\mathrm{MnO}_{2} /$ polypyrrole nanorod composites for high-performance supercapacitors. J. Mater. Chem. 21, 1096510969 (2011).

15 Wang, X. \& Li, Y. Selected-control hydrothermal synthesis of $\alpha$-and $\beta-\mathrm{MnO}_{2}$ single crystal nanowires. J. Am. Chem. Soc 124, 2880-2881 (2002).

16 Wang, X. \& Li, Y. Synthesis and formation mechanism of manganese dioxide nanowires/nanorods. Chemistry-A European Journal 9, 300-306 (2003).

17 Subramanian, V., Zhu, H., Vajtai, R., Ajayan, P. \& Wei, B. Hydrothermal synthesis and pseudocapacitance properties of $\mathrm{MnO}_{2}$ nanostructures. J. Phys. Chem. B 109, 20207-20214 (2005).

18 Portehault, D., Cassaignon, S., Baudrin, E. \& Jolivet, J.-P. Morphology control of cryptomelane type $\mathrm{MnO}_{2}$ nanowires by soft chemistry. Growth mechanisms in aqueous medium. Chem. Mater. 19, 5410-5417 (2007).

19 Huang, X., Lv, D., Yue, H., Attia, A. \& Yang, Y. Controllable synthesis of $\alpha$-and $\beta-\mathrm{MnO}_{2}$ : cationic effect on hydrothermal crystallization. Nanotechnology 19, 225606 (2008).

20 Luo, J. et al. Synthesis of single-crystal tetragonal $\alpha-\mathrm{MnO}_{2}$ nanotubes. J. Phys. Chem. C 112, 12594-12598 (2008).

21 Wang, J. et al. Fluoride anions-assisted hydrothermal preparation and growth process of $\beta-\mathrm{MnO}_{2}$ with bipyramid prism morphology. CrystEngComm 15, 66826689 (2013).

22 Yuan, Y. et al. Asynchronous Crystal cell expansion during lithiation of $\mathrm{K}^{+}$stabilized $\alpha-\mathrm{MnO}_{2}$. Nano Lett. 15, 2998-3007 (2015).

23 Duan, X. et al. Controllable hydrothermal synthesis of manganese dioxide nanostructures: shape evolution, growth mechanism and electrochemical properties. CrystEngComm 14, 4196-4204 (2012).

24 Zhang, X., Yang, W., Yang, J. \& Evans, D. G. Synthesis and characterization of $\alpha-\mathrm{MnO}_{2}$ nanowires: self-assembly and phase transformation to $\beta-\mathrm{MnO}_{2}$ microcrystals. J. Cryst. Growth 310, 716-722 (2008).

25 Yuan, Y. et al. Atomistic insights into the oriented attachment of tunnel-based oxide nanostructures. ACS Nano 10, 539-548 (2015). 
26 Zhang, X., Yu, P., Wang, D. \& Ma, Y. Controllable synthesis of $\alpha-\mathrm{MnO}_{2}$ nanostructures and phase transformation to $\beta-\mathrm{MnO}_{2}$ microcrystals by hydrothermal crystallization. J. Nanosci. Nanotechnol. 10, 898-904 (2010).

27 Yuan, Y. et al. The influence of large cations on the electrochemical properties of tunnel-structured metal oxides. Nat. Commun. 7, 13374 (2016).

28 Zhang, Y., Chen, L., Zheng, Z. \& Yang, F. A redox-hydrothermal route to $\beta$ $\mathrm{MnO}_{2}$ hollow octahedra. Solid State Sciences 11, 1265-1269 (2009).

29 Yu, P., Zhang, X., Sun, X., Wang, D. \& Ma, Y. One-pot hydrothermal synthesis of $\beta-\mathrm{MnO}_{2}$ crystals and their magnetic properties. J. Phys. Chem. Solids 74, 16261631 (2013).

30 Birgisson, S., Saha, D. \& Iversen, B. B. Formation mechanisms of nanocrystalline $\mathrm{MnO}_{2}$ polymorphs under hydrothermal conditions. Cryst. Growth Des. 18, $827-$ 838 (2018).

31 Schmid, H. \& Mader, W. Oxidation states of Mn and Fe in various compound oxide systems. Micron 37, 426-432 (2006).

32 Wang, D. et al. Crystal facet dependence of water oxidation on $\mathrm{BiVO}_{4}$ sheets under visible light irradiation. Chem. Eur. J. 17, 1275-1282 (2011).

33 Yao, W. et al. Energy-driven surface evolution in beta- $\mathrm{MnO}_{2}$ structures. Nano Res. 11, 206-215 (2018).

34 Tompsett, D. A., Parker, S. C. \& Islam, M. S. Rutile $(\beta-) \mathrm{MnO}_{2}$ surfaces and vacancy formation for high electrochemical and catalytic performance. J. Am. Chem. Soc. 136, 1418-1426 (2014).

35 Maphanga, R., Parker, S. \& Ngoepe, P. Atomistic simulation of the surface structure of electrolytic manganese dioxide. Surf. Sci. 603, 3184-3190 (2009).

36 Tompsett, D. A., Parker, S. C., Bruce, P. G. \& Islam, M. S. Nanostructuring of $\beta$ $\mathrm{MnO}_{2}$ : The important role of surface to bulk ion migration. Chem. Mater. 25, 536-541 (2013).

37 Oxford, G. A. \& Chaka, A. M. First-principles calculations of clean, oxidized, and reduced $\beta-\mathrm{MnO}_{2}$ surfaces. J. Phys. Chem. C 115, 16992-17008 (2011).

38 Horikawa, T., Do, D. \& Nicholson, D. Capillary condensation of adsorbates in porous materials. Adv. Colloid Interface Sci. 169, 40-58 (2011). 
39 Chen, W.-M. et al. Controllable synthesis of hollow bipyramid $\beta-\mathrm{MnO}_{2}$ and its high electrochemical performance for lithium storage. ACS Appl. Mater.

Interfaces 4, 3047-3053 (2012).

40 Tang, W., Yang, X., Liu, Z. \& Ooi, K. Preparation of $\beta-\mathrm{MnO}_{2}$ nanocrystal/acetylene black composites for lithium batteries. J. Mater. Chem. 13, 2989-2995 (2003).

41 David, W., Thackeray, M., Bruce, P. \& Goodenough, J. Lithium insertion into $\beta$ $\mathrm{MnO}_{2}$ and the rutile-spinel transformation. Mater. Res. Bull. 19, 99-106 (1984).

42 Huang, $\mathrm{X}$. et al. Highly crystalline macroporous $\beta-\mathrm{MnO}_{2}$ : hydrothermal synthesis and application in lithium battery. Electrochim. Acta 55, 4915-4920 (2010).

43 Kresse, G. \& Hafner, J. Ab-Initio molecular-dynamics simulation of the liquidmetal amorphous-semiconductor transition in germanium. Phys. Rev. B 49, 14251-14269 (1994).

44 Kresse, G. \& Furthmuller, J. Efficiency of ab-initio total energy calculations for metals and semiconductors using a plane-wave basis set. Comput. Mater. Sci. 6, 15-50 (1996).

45 Kresse, G. \& Furthmuller, J. Efficient iterative schemes for ab initio total-energy calculations using a plane-wave basis set. Phys. Rev. B 54, 11169-11186 (1996).

46 Kresse, G. \& Joubert, D. From ultrasoft pseudopotentials to the projector augmented-wave method. Phys. Rev. B 59, 1758-1775 (1999).

47 Perdew, J. P., Burke, K. \& Ernzerhof, M. Generalized gradient approximation made simple. Phys. Rev. Lett. 77, 3865-3868 (1996).

48 Dudarev, S. L., Botton, G. A., Savrasov, S. Y., Humphreys, C. J. \& Sutton, A. P. Electron-energy-loss spectra and the structural stability of nickel oxide: An LSDA+U study. Phys. Rev. B 57, 1505-1509 (1998).

49 Tompsett, D. A. \& Islam, M. S. Electrochemistry of hollandite alpha-MnO 2 : Liion and Na-ion insertion and $\mathrm{Li}_{2} \mathrm{O}$ incorporation. Chem. Mater. 25, 2515-2526 (2013).

50 Monkhorst, H. J. \& Pack, J. D. Special points for brillouin-zone integrations. Phys. Rev. B 13, 5188-5192 (1976). 


\section{Chapter 6 Facet-dependent electrochemical and catalytic performance of beta $\mathrm{MnO}_{2}$ crystals $^{4}$}

Manganese dioxide $\left(\mathrm{MnO}_{2}\right)$ have wide applications in rechargeable batteries, supercapacitors, and metal-air batteries. The electrochemical and catalytic properties of $\mathrm{MnO}_{2}$ crystals are highly related to their phases and morphologies. In this work, $\beta-\mathrm{MnO}_{2}$ crystals with high occupancies of $\{100\}$ or $\{111\}$ facets were synthesized. As the electrode for supercapacitors, the $\{100\}$ facets offered higher specific capacitance than the $\{111\}$ facets. The capacitance difference was due to higher exposed Mn centers on the $\{100\}$ facets. As the cathode catalyst for Li-air batteries, the $\{111\}$ and $\{100\}$ facets of $\beta-\mathrm{MnO}_{2}$ catalyzed the formation of $\mathrm{Li}_{2} \mathrm{O}_{2}$ into large toroids and thin film, respectively. For the first time, we revealed crystal facets of the catalyst can change the $\mathrm{Li}_{2} \mathrm{O}_{2}$ formation from the surface mechanism to the solution mechanism even in the low donor number TEGDME electrolyte. The different catalytic properties were due to the different adsorption free energies for the $\mathrm{LiO}_{2}$ intermediate on the $\{111\}$ and $\{100\}$ facets. Our findings revealed that facet-engineering of the cathode catalysts would open a new way for the design of lithium-air batteries with high stabilities and long cycle life.

\subsection{Introduction}

Facet engineering offers a unique way to tune the morphologies of crystalline materials and bring superior physical or chemical properties for their different applications. In energy storage devices, exposed crystal facets of the electrode materials closely interact with the

\footnotetext{
${ }^{4}$ The materials contained in this chapter are planned for submission. Copyright transfer agreement will become effective only after being accepted.
} 
surrounding electrolytes and thus directly affect their electrochemical or catalytical performances. For lithium-ion batteries, intercalation-based electrodes often show highly selective pathways for the insertion of lithium ions. The exposed crystal facets directly affect the rate performance of the electrodes. ${ }^{1-4}$ Previous computational and experimental analysis demonstrated the preferred lithium ion diffusion was along the (010) plane for $\mathrm{V}_{2} \mathrm{O}_{5},{ }^{5} \mathrm{LiCoO}_{2},{ }^{6} \mathrm{LiFePO}_{4},{ }^{3,7} \mathrm{LiNi}_{1 / 3} \mathrm{Co}_{1 / 3} \mathrm{Mn}_{1 / 3} \mathrm{O}_{2},{ }^{1} \mathrm{Li}\left[\mathrm{Li}_{1 / 3-2 \times / 3} \mathrm{Ni}_{x} \mathrm{Mn}_{2 / 3-\mathrm{x} / 3}\right] \mathrm{O}_{2},{ }^{2}$ or the [001] tunnel direction for $\alpha-\mathrm{MnO}_{2}, \beta-\mathrm{MnO}_{2}$, etc. ${ }^{8}$ For example, $\mathrm{LiFePO}_{4}$ nanoplates with $a c$ facet orientation gave higher rate performance with a capacity around $148 \mathrm{mAh} \mathrm{g}^{-1}$ at $10 \mathrm{C}$-rate than the nanoplates with $b c$ facet orientation $\left(28 \mathrm{mAh} \mathrm{g}^{-1}\right) \cdot{ }^{3}$ For supercapacitors, exposed facets of the electrode crystals determined the number of active sites for the adsorption/desorption of active cations. $\mathrm{Mn}_{3} \mathrm{O}_{4}$ nano-octahedrons with $\{111\}$ exposed facets exhibited a higher specific capacitance of $322 \mathrm{~F} \cdot \mathrm{g}^{-1}$ than that of the truncatedoctahedrons $\left(244 \mathrm{~F} \cdot \mathrm{g}^{-1}\right){ }^{9}$ For cathode catalysts in metal-air batteries or fuel cells, exposed facets of the catalysts determined the activation energies for the oxygen reduction or evolution reactions (ORR/OER). As the cathode catalyst for Li-air batteries, $\mathrm{Co}_{3} \mathrm{O}_{4}$ with $\{111\}$ facets showed better catalytic performance in promoting the OER process and reducing the charge-discharge overpotential compared with the $\{100\},\{110\}$, and $\{112\}$ facets; the $\{111\}$ facets of $\mathrm{Co}_{3} \mathrm{O}_{4}$ offered the highest surface energies and total density of dangling $\mathrm{Co}^{2+}$ and $\mathrm{Co}^{3+}$ bonds for surface reactions. ${ }^{10}$

Earth-abundant $\mathrm{MnO}_{2}$ crystals have wide applications as the electrode material for rechargeable batteries and supercapacitors, or as bifunctional catalysts for metal-air batteries and fuel cells. Among all the crystal phases of $\mathrm{MnO}_{2}, \beta-\mathrm{MnO}_{2}$ is 
thermodynamically the most stable phase. ${ }^{11}$ The morphology of $\beta-\mathrm{MnO}_{2}$ directly affects its electrochemical and catalytic performance. As the electrode for supercapacitors, $\beta$ $\mathrm{MnO}_{2}$ nanorods showed low capacitance around $28 \mathrm{~F} \cdot \mathrm{g}^{-1} \cdot{ }^{12}$ Ultralong $\beta-\mathrm{MnO}_{2}$ nanowires with highly exposed lateral surfaces offered almost ten times specific capacitance around $279 \mathrm{~F} \cdot \mathrm{g}^{-1} \cdot{ }^{13}$ However, the role of crystal facets for the different specific capacitance of $\beta$ $\mathrm{MnO}_{2}$ is still unknown. As the effective, non-precious, and bifunctional cathode catalysts, $\mathrm{MnO}_{2}$-based structures received lots of attention. ${ }^{14}$ The effect of crystal phases and morphologies of $\mathrm{MnO}_{2}$ was broadly evaluated as the cathode catalyst for Li-air batteries. ${ }^{14-}$ ${ }^{18}$ The catalytic performance of $\mathrm{MnO}_{2}$ can be affected by its poor electron conductivity. Therefore, lots of studies focused on improving the conductivity of the $\mathrm{MnO}_{2}$ catalyst through the support of conductive substrate (such as graphene ${ }^{19,20}$, carbon nanotubes, ${ }^{21,22}$, $\mathrm{Ni}$ foam ${ }^{23}$ ) or doping with $\mathrm{Pd},{ }^{24,25} \mathrm{Pt}^{26}{ }^{21},{ }^{27}$ etc. Although $\mathrm{MnO}_{2}$ has been demonstrated to be an effective cathode catalyst, the role of the $\mathrm{MnO}_{2}$ during the electrochemical formation and oxidation of $\mathrm{Li}_{2} \mathrm{O}_{2}$ is still not fully understood. Applying $\beta-\mathrm{MnO}_{2}$ as ORR/OER catalysts, several groups have predicted the different catalytic performance of $\beta-\mathrm{MnO}_{2}$ with different exposed facets based on Density Functional Theory (DFT) simulation. Tompsett et al. ${ }^{28}$ calculated the surface energies and the oxygen vacancy formation energies on different facets of rutile $\mathrm{MnO}_{2}$. Dawson et al. ${ }^{29}$ studied the effect of grain boundaries on the formation of oxygen vacancies and predicted higher catalytic activities at the $\beta-\mathrm{MnO}_{2}$ $\Sigma 5(210) /[001]$ grain boundaries than the bulk. Li et al..$^{30}$ specifically studied the effect of oxygen vacancy concentration on the catalytic performance of the $\beta-\mathrm{MnO}_{2}$ (110) facet. Mellan et al. ${ }^{31}$ studied the lithium and oxygen adsorption on the (110) facet of $\beta-\mathrm{MnO}_{2}$. Although the catalytic performance of beta $\mathrm{MnO}_{2}$ for oxygen reduction and evolution has 
been predicted theoretically, the simulation conditions are generally ideal. Experimental efforts are still needed to achieve controllable facet engineering during material synthesis and to further explore the performance of beta $\mathrm{MnO}_{2}$ in the complex environment of real devices.

In this paper, $\beta-\mathrm{MnO}_{2}$ crystals with different occupancy of $\{111\}$ and $\{100\}$ facets were synthesized following a hydrothermal method. The effect of crystal facets on the performance of $\beta-\mathrm{MnO}_{2}$ crystals was thoroughly evaluated based on its application as the electrode for supercapacitors as well as the cathode for Li-air batteries. For supercapacitor application, the $\{100\}$ facets of $\beta-\mathrm{MnO}_{2}$ crystals provided higher specific capacitance than the $\{111\}$ facets. As the cathode catalyst for Li-air batteries, both facets reduced the chargedischarge overpotentials than pure carbon cathode. The $\{111\}$ facets facilitated the solution mechanism for the generation of $\mathrm{Li}_{2} \mathrm{O}_{2}$ toroids in the tetraethylene glycol dimethyl ether (TEGDME) electrolyte with low donor numbers (DN).

\subsection{Results and Discussion}

\subsubsection{Materials synthesis and characterization}

$\beta-\mathrm{MnO}_{2}$ crystals were synthesized based on the redox reaction between $\mathrm{MnO}_{4}{ }^{-}$and $\mathrm{Mn}^{2+}$. The morphology of the crystals was controlled used different concentrations of $\mathrm{KCl}$. The obtained crystals with $0.03 \mathrm{M}$ and $0.09 \mathrm{M} \mathrm{KCl}$ showed bipyramid prism and octahedron shapes, respectively (Fig. 6-1a, b). The phase of the crystals was confirmed to be $\beta-\mathrm{MnO}_{2}$ based on their powder X-ray diffraction (XRD) patterns. The XRD patterns were in good agreement with standard JCPDS 24-0735 with lattice parameters $a=b=4.399 \AA, c=$ 
2.874A. Following the morphology change from bipyramid prism to the octahedron, the ratio of integrated XRD peak intensity $\mathrm{I}_{(111)} / \mathrm{I}_{(200)}$ increased from 0.514 to 2.001 , with a clear change of peak intensities (indicated by the arrows in Fig. 6-1c).
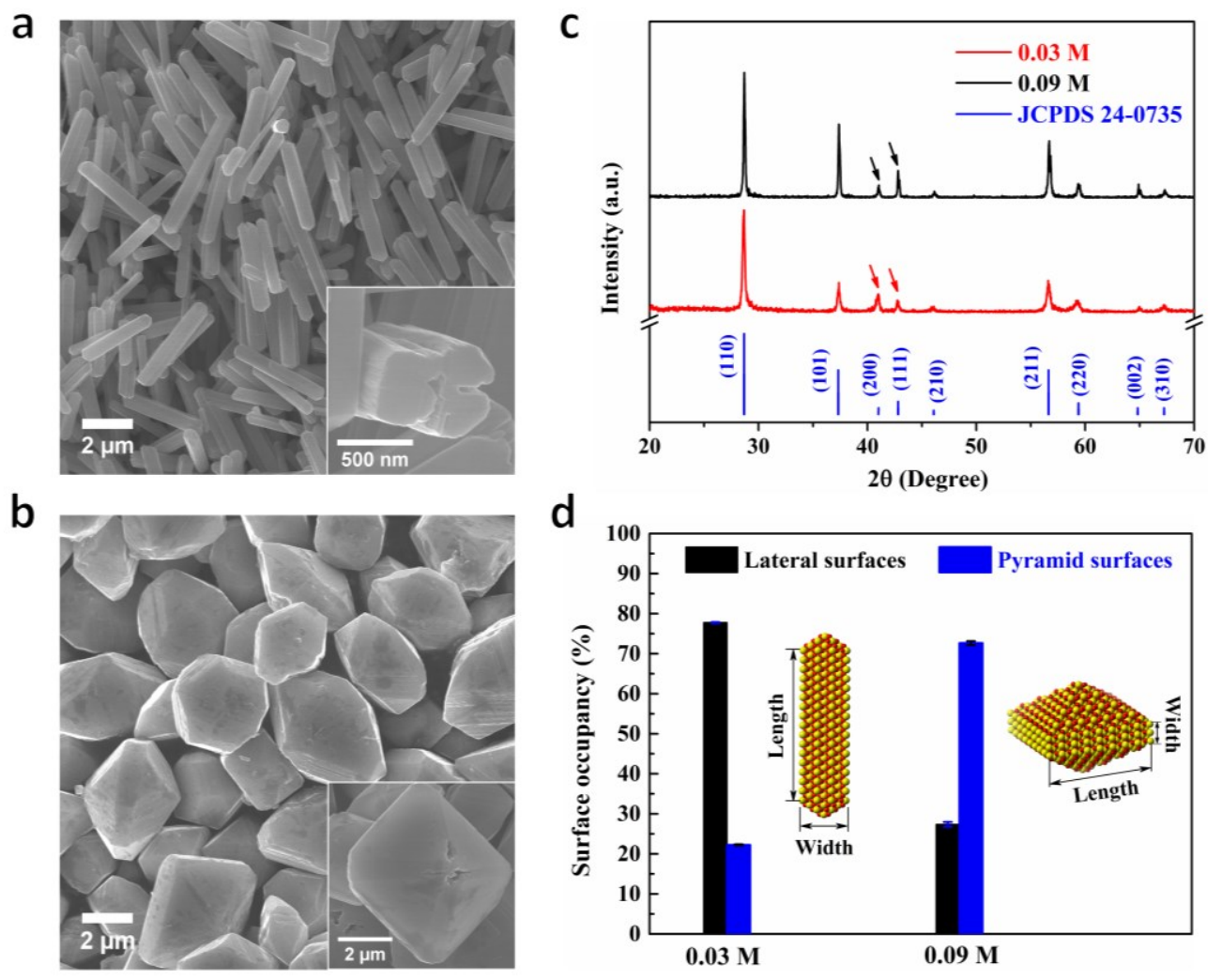

Figure 6-1 FE-SEM images of the $\beta-\mathrm{MnO}_{2}$ crystals with (a) bipyramid prism and (b) octahedron structure obtained with $0.03 \mathrm{M}$ and $0.09 \mathrm{M} \mathrm{KCl}$, respectively. (c) XRD patterns of the beta $\mathrm{MnO}_{2}$ crystals in agreement with $\beta-\mathrm{MnO}_{2}$ standard JCPDS 24-0736. (d) Averaged occupancy of lateral and pyramid surfaces in the two morphologies of $\beta-\mathrm{MnO}_{2}$ crystals.

Statistical analysis was performed to show the morphology change from the bipyramid prism to the octahedron. The length and width of the prism part were analyzed specifically (Fig. 6-1d). The bipyramid prism morphology showed a prism length close to 
$3.11 \pm 0.34 \mu \mathrm{m}$ and a prism width around $0.36 \pm 0.04 \mu \mathrm{m}$; while the octahedron structure showed a prism length around $0.67 \pm 0.08 \mu \mathrm{m}$ and a prism width around $2.78 \pm 0.27 \mu \mathrm{m}$. The distributions of these dimensions were summarized in Fig. S1. The occupancy of lateral surfaces and pyramid surfaces was quantified based on these dimensions (Fig. 61d). The occupancy of lateral surfaces was close to $78 \%$ in the bipyramid prism structure, while the occupancy of pyramid surfaces was close to $73 \%$ in the octahedron structure. More attention, therefore, was drawn to the indexing of lateral surfaces of the bipyramid prism structure and the pyramid surfaces of the octahedron structure.

The surfaces of the bipyramid prism and the octahedron morphologies were identified combining high-resolution transmission electron microscopy (HR-TEM), crosssection analysis, and scanning electron microscopy (SEM) analysis. The growth direction of the $\beta-\mathrm{MnO}_{2}$ crystal was confirmed to be along the [001] tunnel direction based on the selected area electron diffraction (SAED) pattern acquired from the [100] zone axis (Fig. 6-2a). To identify the lateral surfaces of the bipyramid prism structure, cross-section samples were prepared by ultramicrotomy. The lateral surfaces of the bipyramid prism were confirmed to be the $\{100\}$ surfaces based on the SAED pattern acquired from the [001] tunnel axis (Fig. 6-2b). The lateral surfaces of the octahedron were confirmed to be the $\{110\}$ surfaces based on the SAED pattern obtained from the same [001] zone axis (Fig. 6-2c). The shift of lateral surfaces from $\{100\}$ to $\{110\}$ is consistent with previous studies, ${ }^{32}$ which is due to the higher surface energy of $\{100\}$ facets $\left(0.94 \mathrm{~J} / \mathrm{m}^{2}\right)$ compared with $\{110\}\left(0.62 \mathrm{~J} / \mathrm{m}^{2}\right) .{ }^{28}$ Atom model of the $\beta-\mathrm{MnO}_{2}$ crystal viewed along the tunnel direction with $\{110\}$ lateral surfaces was shown in Fig. 6-2d. To confirm the pyramid 
surfaces in the bipyramid prism and the octahedron structures belong to $\{111\}$ facets, interplanar angles were measured in the SEM images (Fig. 6-2e, f) and compared with theoretical values, following our previous methods. ${ }^{33}$ The interplanar angle between the pyramid surfaces and $\{110\}$ facets, and the diagonal slant angle of pyramid surfaces were obtained and compared to theoretical values of $132.7^{\circ}$ and $113.6^{\circ}$, respectively. ${ }^{33}$ Representative measurements performed on both morphologies were shown in Fig. 2e and Fig. 6-2f. 

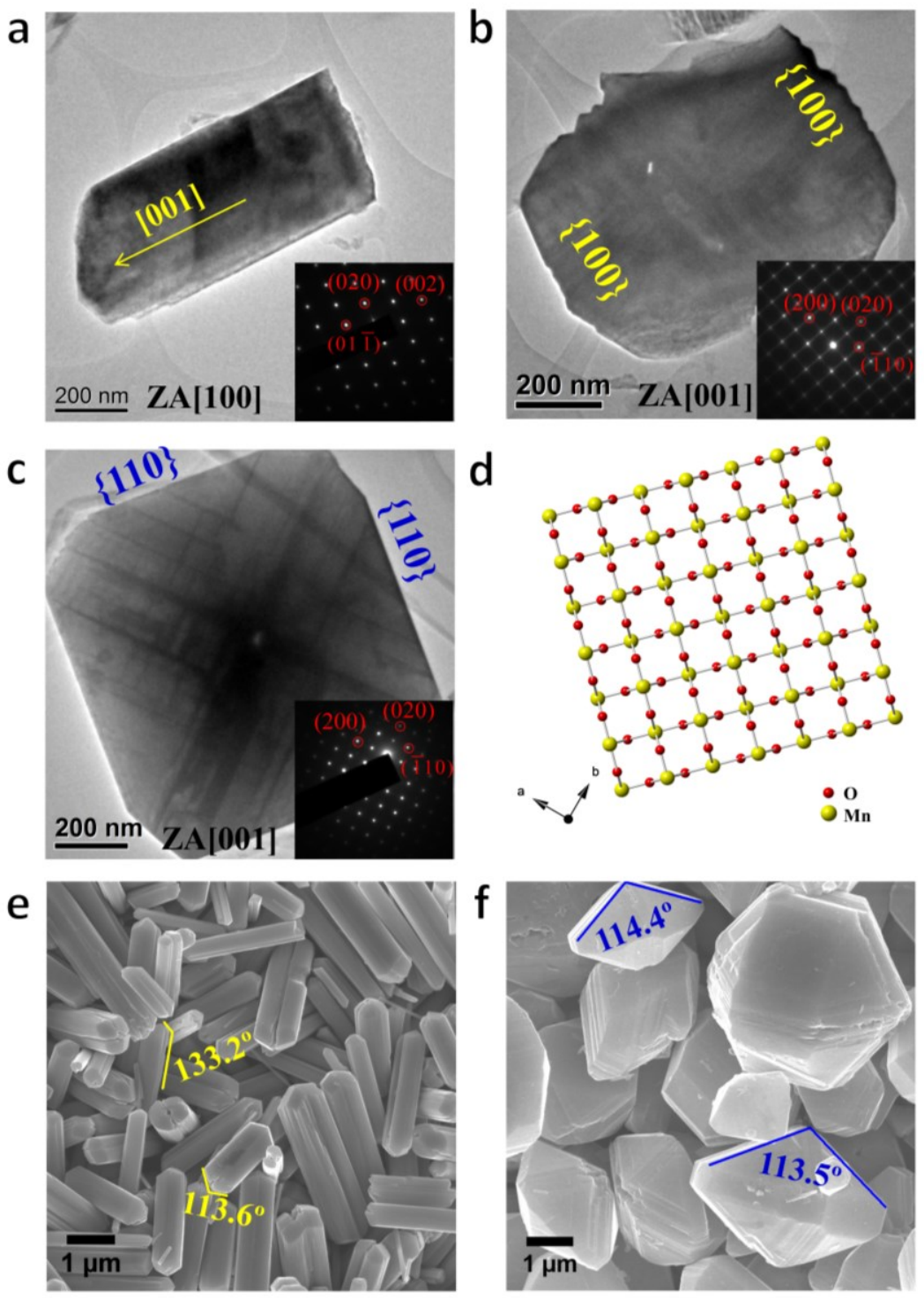

Figure 6-2 Identification of the lateral and pyramid surfaces of the two $\beta-\mathrm{MnO}_{2}$ morphologies combining TEM and FE-SEM. (a) $\beta-\mathrm{MnO}_{2}$ bipyramid prism structure viewed along the [100] zone axis confirming the growth direction of the structure is along the [001] tunnel direction. The inset panel gives the corresponding SAED pattern. (b) $\beta$ $\mathrm{MnO}_{2}$ bipyramid prism cross-section sample viewed along the [001] tunnel direction. The inset SAED pattern confirmed the lateral surfaces to be the $\{100\}$ facets. (c) $\beta-\mathrm{MnO}_{2}$ octahedra viewed along the [001] tunnel direction. The inset SAED pattern indicated the lateral surfaces belong to $\{110\}$ facets. (d) The simulated atom model showing the structure of $\beta-\mathrm{MnO}_{2}$ viewed along [001] direction with $\{110\}$ as the lateral surfaces. Representative 
interplanar angle measurements performed in $\beta-\mathrm{MnO}_{2}$ (e) bipyramid prism and (f) octahedron structures.

Based on our previous work, ${ }^{32-34}$ it should be emphasized that the identification of lateral surfaces for nanowires is only reliable based on the TEM image and SAED pattern obtained from the growth direction, in our case, the [001] tunnel direction or the $\{001\}$ zone axis. Using SAED patterns acquired from lateral zone axis to identify lateral facets would be misleading. Because TEM images are projected images and the choose of lateral zone axis (tilting the nanowire) will greatly affect the lateral atoms that one would capture in TEM images. As shown in Fig. S6-2, beta $\mathrm{MnO}_{2}$ with $\{100\}$ lateral facets viewed from [100], [110], and [210] lateral zone axis gives completely different arrangement of lateral Mn atoms. Therefore, the TEM image obtained from lateral zone axis, in most cases, cannot give reliable information for the identification of lateral facets.

\subsubsection{Facets effect on the electrochemical performance of $\beta-\mathrm{MnO}_{2}$}

The effect of crystal facets on the electrochemical performance of $\beta-\mathrm{MnO}_{2}$ crystals was investigated based on their application as the electrode for symmetric supercapacitors. Fig. 6-3a summarized the cyclic voltammogram (CV) curves of the $\beta-\mathrm{MnO}_{2}$ bipyramid prism crystals $(0.03 \mathrm{M}-\{100\})$ symmetric supercapacitor obtained in a $1 \mathrm{M} \mathrm{Na}_{2} \mathrm{SO}_{4}$ aqueous electrolyte under different scan rates ranging from $5 \mathrm{mv} / \mathrm{s}$ to $100 \mathrm{mv} / \mathrm{s}$. All the $\mathrm{CV}$ curves showed symmetric and rectangular shape, indicating ideal pseudocapacitive of the crystals. The galvanostatic charge-discharge curves of the bipyramid prism $\beta-\mathrm{MnO}_{2}$ were acquired under different current densities ranging from $10 \mathrm{~mA} / \mathrm{g}$ to $200 \mathrm{~mA} / \mathrm{g}$ (Fig. 6-3b). For $\beta$ - 
$\mathrm{MnO}_{2}$ octahedron structure $(0.09 \mathrm{M}-\{111\})$, the $\mathrm{CV}$ curves and charge-discharge curves were also obtained under the same scan rates and current densities (Fig. S6-3a, b). The corresponding $\mathrm{CV}$ curves of the octahedron also showed ideal rectangular shape. Fig. 6-3c further compared the $\mathrm{CV}$ curves of the bipyramid prism and octahedron $\beta-\mathrm{MnO}_{2}$ morphologies under the same scan rate of $100 \mathrm{mv} / \mathrm{s}$. The area of the $\mathrm{CV}$ curve of bipyramid prism was clearly larger than that of the octahedron morphology, indicating higher specific capacitance of the bipyramid prism morphology. The specific capacitance for the bipyramid prism structure was found to be $9.2 \mathrm{~F} / \mathrm{g}$ compared with $1.7 \mathrm{~F} / \mathrm{g}$ for the octahedron structure under the same scan rate of $5 \mathrm{mv} / \mathrm{s}$ (Fig. S6-3c). Fig. 6-3d compared the charge-discharge curves of the bipyramid prism and the octahedron under a current density of $10 \mathrm{~mA} / \mathrm{g}$. Longer discharge time was observed for the bipyramid prism structure. The change of specific capacitance versus different scan rate and different current densities were summarized in Fig. S6-3c, d. 

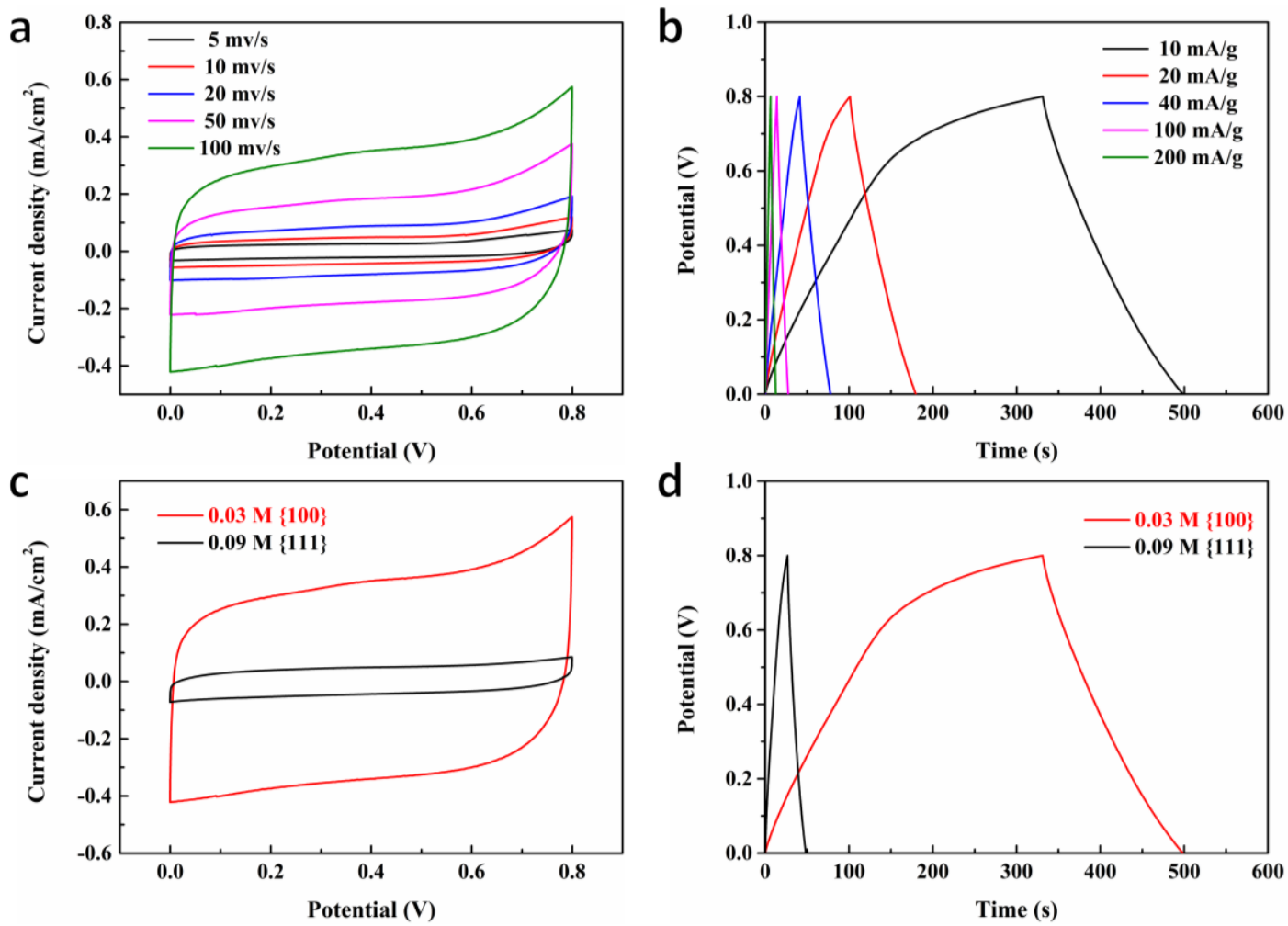

Figure 6-3 (a) Cyclic voltammogram (CV) and (b) galvanostatic charge-discharge curves of the symmetric supercapacitor fabricated from $\beta-\mathrm{MnO}_{2}$ bipyramid prism $(0.03 \mathrm{M}$ $\{100\})$. Different scan rates and current densities were adopted and all the tests were performed in the $1 \mathrm{M} \mathrm{Na}_{2} \mathrm{SO}_{4}$ electrolyte. Comparison between the (c) $\mathrm{CV}$ curves (scan rate $100 \mathrm{mv} / \mathrm{s}$ ) and (d) charge-discharge curves (current density $10 \mathrm{~mA} / \mathrm{g}$ ) of the symmetric supercapacitors build with $\beta-\mathrm{MnO}_{2}$ bipyramid prism $(0.03 \mathrm{M}-\{100\})$ and octahedron $(0.09 \mathrm{M}-\{111\})$ crystals, respectively.

The higher specific capacitance of the bipyramid prism can be related to the higher achievable Mn centers on the $\{100\}$ facets than that on the $\{111\}$ facets. The charge storage for $\beta-\mathrm{MnO}_{2}$-based supercapacitors with an aqueous $\mathrm{Na}_{2} \mathrm{SO}_{4}$ electrolyte followed the reaction:

$$
\mathrm{MnO}_{2}+\mathrm{M}^{+}+\mathrm{e}^{-} \Leftrightarrow \mathrm{MnOOM}
$$


where $\mathrm{M}$ represents hydrated protons $\left(\mathrm{H}_{3} \mathrm{O}^{+}\right)$or $\mathrm{Na}^{+}$. The general reaction mainly involved the adsorption/desorption of $\mathrm{H}_{3} \mathrm{O}^{+}$or $\mathrm{Na}^{+}$cations on the crystal surfaces of $\beta-\mathrm{MnO}_{2}$. Cations intercalation was not considered because the small tunnel size of $\beta-\mathrm{MnO}_{2}(1.89 \AA)$ is not accessible for large hydrated protons or sodium ions. ${ }^{12,35}$ The adsorption/desorption process involved electron transfer and manganese valence state change from $\mathrm{Mn}^{4+}$ to $\mathrm{Mn}^{3+}$. Thus, the different specific capacitances for different $\beta-\mathrm{MnO}_{2}$ morphologies were related to the effective Mn centers offered for cations adsorption/desorption. ${ }^{36}$ The accessible Mn atom densities on different crystal facets of $\beta-\mathrm{MnO}_{2}$ crystals were calculated to be 11.4, 11.2 , and $14.0 \mathrm{~nm}^{-2}$ for the $\{111\},\{110\}$, and $\{100\}$ facets, respectively. The higher density of exposed Mn atoms on the $\{100\}$ lateral facets shall explain the higher specific capacitance obtained from the $\beta-\mathrm{MnO}_{2}$ bipyramid prism morphology. This also demonstrates the previous observation that $\beta-\mathrm{MnO}_{2}$ nanowires with a high aspect ratio and small diameter can provide higher specific capacitance. ${ }^{13}$

To evaluate the effect of surface area on the specific capacitance, BrunauerEmmett-Teller (BET) surface areas of these two morphologies were analyzed (Fig. S6-4). The surface areas were found to be $1.33 \mathrm{~m}^{2} / \mathrm{g}$ and $3.7 \mathrm{~m}^{2} / \mathrm{g}$ for the bipyramid prism and the octahedron morphologies, respectively. The higher surface area of the octahedron morphology (rich in $\{111\}$ facets) did not give higher specific capacitance. This further demonstrated the specific capacitance was mainly related to the effective Mn atoms exposed on different crystal facets. 


\subsubsection{Facets effect on the catalytic performance of $\beta-\mathrm{MnO}_{2}$}

The effect of crystal facets on the catalytic activities of $\beta-\mathrm{MnO}_{2}$ as the cathode catalyst for lithium-air batteries was also evaluated. Fig. 6-4a showed the components of the assembled Li-air cell. Non-aqueous TEGDME electrolyte was adopted because of its excellent stability towards highly reactive oxygen radical anion $\mathrm{O}_{2}-37$ Compared with pure carbon cathode, the two morphologies of $\beta-\mathrm{MnO}_{2}$ crystals clearly reduced both the charging and discharging overpotentials of the cell (Fig. 6-4b). The $\{100\}$ and $\{111\}$ facets showed comparable effect during the battery discharge. The discharge overpotential was reduced around $0.11 \mathrm{~V}$. During charging, the $\{100\}$ facets offered relatively lower overpotential compared with the $\{111\}$ facets.

To better understand the role of $\beta-\mathrm{MnO}_{2}$ crystal facets during the charge-discharge process, the discharge products after the initial discharge to $2.4 \mathrm{~V}$ were examined using SEM (Fig. 6-4c, d). The discharge product catalyzed by the $\{100\}$ facets of $\beta-\mathrm{MnO}_{2}$ (bipyramid prism) showed a film morphology (Fig. 6-4c), while the discharge product catalyzed by the $\{111\}$ facets (octahedron structure) showed a toroidal morphology (Fig. 6-4d).

The ideal electrochemical reaction on the cathode side followed the Equation (6-2):

$$
2 \mathrm{Li}+\mathrm{O}_{2}+2 \mathrm{e}^{-} \underset{\text { Charge }}{\stackrel{\text { Discharge }}{\rightleftarrows}} \mathrm{Li}_{2} \mathrm{O}_{2}
$$

The oxygen reduction to $\mathrm{Li}_{2} \mathrm{O}_{2}$ at low overpotentials has two mechanisms: the solution mechanism, where $\mathrm{Li}_{2} \mathrm{O}_{2}$ forms mainly in the solution as large particles or toroids; and the surface mechanism, where $\mathrm{Li}_{2} \mathrm{O}_{2}$ forms as thin films on the surface of the cathode. ${ }^{38}$ The 
mechanism of $\mathrm{Li}_{2} \mathrm{O}_{2}$ formation was found to be governed by the competition between the $\mathrm{LiO}_{2}$ solubility in the electrolyte and the adsorption free energy of the $\mathrm{LiO}_{2}$ intermediate on the electrode surfaces. ${ }^{39,40}$ The solubility of $\mathrm{LiO}_{2}$ in the electrolyte can be increased using solvent molecules with high donor numbers. ${ }^{41}$ Highly dissolved $\mathrm{LiO}_{2}$ intermediate in the electrolyte can facilitate the solution mechanism for growth of $\mathrm{Li}_{2} \mathrm{O}_{2}$ into large particles, which is beneficial for the capacity and cycle life of the Li-air cell. ${ }^{39}$

The TEGDME electrolyte used here has a relatively low donor number (16.6). ${ }^{42}$ The solubility of $\mathrm{LiO}_{2}$ intermediate would be low in this electrolyte. ${ }^{39}$ A surface mechanism should have dominated the $\mathrm{Li}_{2} \mathrm{O}_{2}$ formation process according to current theory. However, our observation showed that, while the $\beta-\mathrm{MnO}_{2}$ bipyramid prism followed the surface mechanism, the solution mechanism was surprisingly dominant on the carbon cathode loaded with $\beta-\mathrm{MnO}_{2}$ octahedrons ( $\{111\}$ facets). The reason could be related to the competition between the $\mathrm{LiO}_{2}$ solubility and its adsorption free energy on different $\beta-\mathrm{MnO}_{2}$ facets. With the same electrolyte, the solubility of $\mathrm{LiO}_{2}$ should be consistent in the two cells catalyzed with the two different morphologies. Therefore, the appearance of solution mechanism should be related to the different adsorption free energies of $\mathrm{LiO}_{2}$ intermediate on the $\{111\}$ and $\{100\}$ facets of $\beta-\mathrm{MnO}_{2}$. (simulation requested) Lower adsorption free energies would be expected for the $\{100\}$ facets of $\beta$ $\mathrm{MnO}_{2}$, which shall promote the capture of $\mathrm{LiO}_{2}$ intermediate and facilitate the surface mechanism to form $\mathrm{Li}_{2} \mathrm{O}_{2}$ thin films on the electrode surface. Higher adsorption free energies would then be expected for the $\{111\}$ facets of $\beta-\mathrm{MnO}_{2}$, which shall suppress the 
attachment of $\mathrm{LiO}_{2}$ intermediate to the surface and thus facilitate the solution mechanism to form $\mathrm{Li}_{2} \mathrm{O}_{2}$ toroids.

On the other hand, the adsorption of $\mathrm{LiO}_{2}$ intermediate could be affected by the vacancies on the electrode surface. Oxygen radical anion $\mathrm{O}_{2}{ }^{-}$is needed for the generation of $\mathrm{LiO}_{2}$ intermediate. The formation of $\mathrm{O}_{2}{ }^{-}$follows the Equation (6-3), where $\mathrm{O}_{2}$ molecules are adsorbed on the vacancies of the electrode surfaces and take one electron to form $\mathrm{O}_{2}{ }^{-}$ anions. $^{43}$

$$
\mathrm{O}_{2 \text { (sol) }}+\mathrm{e}^{-}+* \text { (vacancies) } \rightarrow \mathrm{O}_{2}^{-} \text {(ad) }
$$

Previous Density Functional Theory (DFT) simulation has shown the formation energies of oxygen vacancies on the $\{100\}$ and $\{111\}$ facets of $\beta-\mathrm{MnO}_{2}$ were 1.13 and $1.41 \mathrm{eV}$, respectively. ${ }^{28}$ The lower oxygen vacancies formation energy on $\{100\}$ facets would promote the adsorption of dissolved $\mathrm{O}_{2}$ molecules and formation of $\mathrm{O}_{2}{ }^{-}$anions. This in return would affect the adsorption of $\mathrm{LiO}_{2}$ intermediate. 

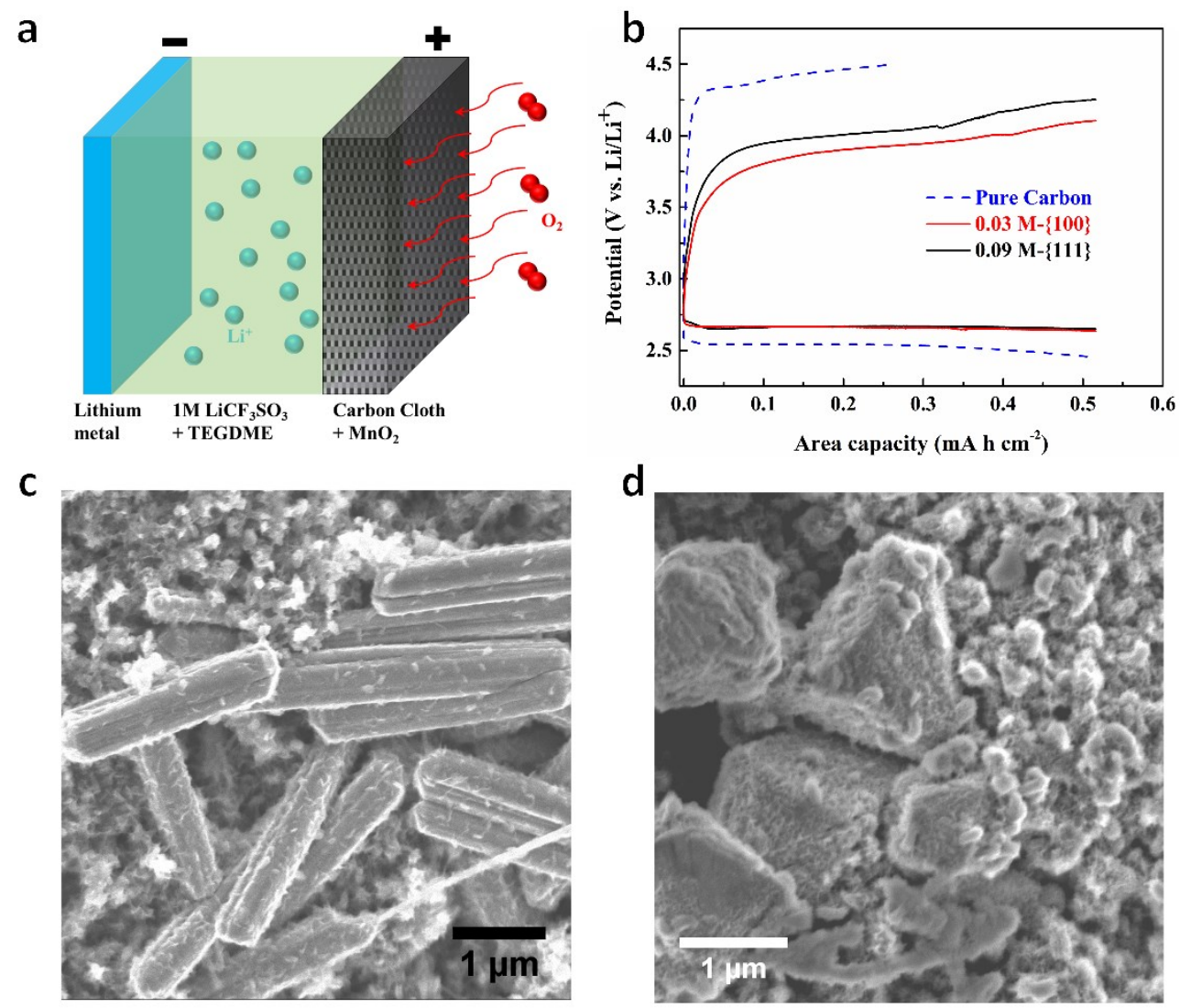

Figure 6-4 (a) Schematic image showing the structure of the Swagelok-type cell. (b) The first cycle charge-discharge curves of the Li-air cells composed of pure carbon, $\beta-\mathrm{MnO}_{2}$ bipyramid prism $(0.03 \mathrm{M}-\{100\})$, and $\beta-\mathrm{MnO}_{2}$ octahedron $(0.09 \mathrm{M}-\{111\})$ cathode. SEM images showing the morphologies of the discharge products on the surface of (c) $\beta-\mathrm{MnO}_{2}$ bipyramid prism and (f) octahedron.

To better illustrate the role of different crystal facets of $\beta-\mathrm{MnO}_{2}$ during the formation of $\mathrm{Li}_{2} \mathrm{O}_{2}$, the reaction processes were further summarized and shown in Fig. 65. The $\mathrm{LiO}_{2}$ intermediate was preferred to be absorbed by the $\{100\}$ facets. The adsorbed $\mathrm{LiO}_{2}$ then followed a second electron reduction or direct disproportionate to generate $\mathrm{Li}_{2} \mathrm{O}_{2}$ 
thin films. The $\{111\}$ facets of $\beta-\mathrm{MnO}_{2}$, however, facilitated solution-based mechanism, where $\mathrm{LiO}_{2}$ was mainly dissolved in the electrolyte. The dissolved $\mathrm{LiO}_{2}$ then followed a direct disproportionate or a second electron reduction to form large $\mathrm{Li}_{2} \mathrm{O}_{2}$ toroids.

During the oxidation of $\mathrm{Li}_{2} \mathrm{O}_{2}$, the thin film oxidation was generally facile; while the decomposition of large $\mathrm{Li}_{2} \mathrm{O}_{2}$ particles was a challenge because the large particles are generally remote from the electrode surfaces and the $\mathrm{Li}_{2} \mathrm{O}_{2}$ surfaces showed insulating behavior. ${ }^{44}$ This explains our observation that higher charging overpotential was detected in the Li-air cell catalyzed by $\{111\}$ facets of $\beta-\mathrm{MnO}_{2}$, where $\mathrm{Li}_{2} \mathrm{O}_{2}$ toroids were generated.

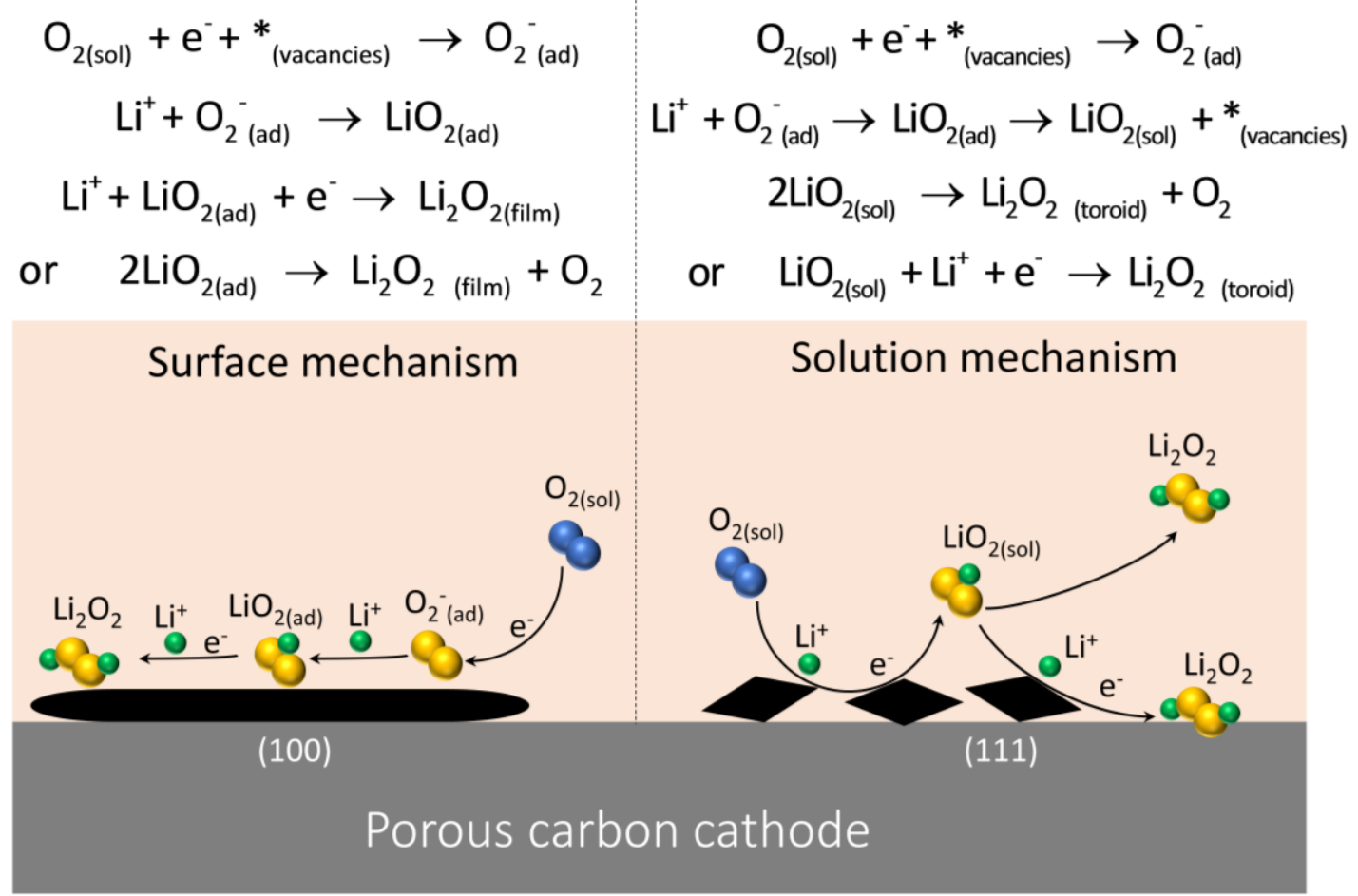

Figure 6-5 Proposed oxygen reduction mechanisms on the carbon cathode catalyzed by $\beta$ $\mathrm{MnO}_{2}$ bipyramid prism $(0.03 \mathrm{M}-\{100\})$ and octahedron $(0.09 \mathrm{M}-\{111\})$ crystals. 


\subsection{Conclusions}

In summary, $\beta-\mathrm{MnO}_{2}$ crystals with highly exposed $\{100\}$ and $\{111\}$ facets were synthesized to evaluate the effect of crystal facets on its electrochemical and catalytic performances. As the electrode for supercapacitors, the $\{100\}$ lateral facets of $\beta-\mathrm{MnO}_{2}$ offered the crystal much higher specific capacitance than the $\{111\}$ pyramid facets. This explains previous observations that ultralong nanowires of $\beta-\mathrm{MnO}_{2}$ exhibited ten times higher capacitance than traditional rod structure. The high specific capacitance of the $\{100\}$ facets was related to their higher exposed Mn centers compared with $\{110\}$ and $\{111\}$ facets. Applying the $\beta-\mathrm{MnO}_{2}$ crystals as the cathode catalyst for lithium-air batteries showed clear improvements in reducing the charge and discharge overpotential compared with the bare carbon cathode. The $\{111\}$ facets of $\beta-\mathrm{MnO}_{2}$ facilitated the solution mechanism for the formation of $\mathrm{Li}_{2} \mathrm{O}_{2}$ toroids; the $\{100\}$ facets, however, catalyzed the surface mechanism for the generation of $\mathrm{Li}_{2} \mathrm{O}_{2}$ films. The effect of different crystal facets of $\beta-\mathrm{MnO}_{2}$ on the formation of $\mathrm{Li}_{2} \mathrm{O}_{2}$ showed that it is possible to induce solution-based mechanism for the oxygen reduction to $\mathrm{Li}_{2} \mathrm{O}_{2}$ even in a low donor number TEGDME electrolyte. The different catalytic performance may due to different adsorption free energies for the $\mathrm{LiO}_{2}$ intermediate on the $\{100\}$ and $\{111\}$ facets. Our findings indicate high donor number electrolytes are not the only choice for high-performance lithium-air batteries. Facet-engineering of the cathode catalysts could open a new way for the selection of stable low DN electrolytes without scarifying the capacity and cycle life of the Li-air batteries. 


\subsection{Experimental}

\subsubsection{Materials Synthesis}

All chemicals were purchased from Sigma-Aldrich and used as received. $\beta-\mathrm{MnO}_{2}$ crystals were synthesized following a reported hydrothermal method. ${ }^{33}$ First, two parallel solutions were prepared by dissolving $0.08 \mathrm{M} \mathrm{KMnO}_{4}$ and $0.12 \mathrm{M} \mathrm{MnCl}_{2} \cdot 4 \mathrm{H}_{2} \mathrm{O}$ in $80 \mathrm{~mL}$ DI water. Then, $0.03 \mathrm{M}$ and $0.09 \mathrm{M} \mathrm{KCl}$ were introduced respectively under constant stirring. The two mixtures were stirred continuously for $30 \mathrm{~min}$ before transferring into $100 \mathrm{~mL}$ Teflonlined stainless-steel autoclaves. The autoclaves were sealed and heated at $180{ }^{\circ} \mathrm{C}$ for $12 \mathrm{~h}$. The obtained $\mathrm{MnO}_{2}$ crystals were collected through vacuum filtration and then washed with DI water and ethyl alcohol for three times, respectively. The final products were dried in air at $60^{\circ} \mathrm{C}$ for $12 \mathrm{~h}$.

\subsubsection{Materials Characterization}

The structure of the synthesized $\mathrm{MnO}_{2}$ was first examined using a Scintag XDS2000 powder X-ray diffractometer equipped with the $\mathrm{Cu} K \alpha$ radiation $(\lambda=1.54056 \AA)$. The scan step was $0.02^{\circ} \mathrm{sec}^{-1}$ with $2 \theta$ ranging from $10^{\circ}$ to $70^{\circ}$. The morphology of the crystals was examined using Hitachi S-4700 field emission scanning electron microscope (FE-SEM). High-resolution transmission electron microscopy (HRTEM, JEOL 3010) was used to determine the growth direction as well as the exposed facets of the crystal structures. For this microscope, the angle between directions in the image and the directions in the diffraction pattern is $0^{\circ}$. BET surface areas of the crystals were measured by $\mathrm{N}_{2}$ sorption isotherms at $77 \mathrm{~K}$ using a Micromeritics ASAP2020 analyzer. The morphology of the 
discharge products in Li-air batteries cathode was examined using JEOL JSM-6320F field emission SEM.

\subsubsection{Cross section sample preparation}

To clearly reveal the crystal facets of the $\beta-\mathrm{MnO}_{2}$ structures, cross section samples were prepared by ultramicrotomy. The obtained powder samples were first mixed with cold mounting epoxy resin (EPOFIX, Electron Microscopy Sciences) and hardener (EPOFIX, Triethylenetetramine, Electron Microscopy Sciences) in a 15:2 ratio under ultrasonication. The mixture was then transferred to a multi-well embedding mold for solidification in the air at $60^{\circ} \mathrm{C}$ for $24 \mathrm{~h}$. Cross-section samples were cut by mounting the samples on a Leica Ultracut UCT ultramicrotome and slicing with a diamond knife with a feeding step size of $500 \mathrm{~nm}$.

\subsubsection{Electrochemical performance test}

The electrode slurry was prepared by mixing the $\beta-\mathrm{MnO}_{2}$ crystals, super $\mathrm{P}$, and PVDF with a mass ratio of 5:3:2 in N-methyl-2pyrrolidone (NMP) solvent. The obtained slurry was then coated on a carbon paper and dried under vacuum for $12 \mathrm{~h}$. For supercapacitor test, a typical two-electrode coin cell (CR2032, MTI) was used to prepare symmetric capacitors with the $\beta-\mathrm{MnO}_{2}$ mixture as the electrode. A glass fiber separator was used. The cells were assembled in the air with $1 \mathrm{M} \mathrm{Na}_{2} \mathrm{SO}_{4}$ as the liquid electrolyte. The cyclic voltammetry (CV) and galvanostatic charge-discharge tests were performed on a multichannel electrochemical workstation (BioLogic). The $\mathrm{CV}$ test was performed from $0 \mathrm{~V}$ to $0.8 \mathrm{~V}$ 
with a scan rate ranging from $5 \mathrm{mV} / \mathrm{s}$ to $100 \mathrm{mV} / \mathrm{s}$. The charge/discharge test was performed with a current density ranging from $10 \mathrm{~mA} / \mathrm{g}$ to $200 \mathrm{~mA} / \mathrm{g}$.

For the lithium-air battery, the electrochemical characterization was carried out using a Swagelok-type cell composed of a lithium metal anode, electrolyte (a glass fiber separator impregnated with $1 \mathrm{M} \mathrm{LiCF}_{3} \mathrm{SO}_{3}$ in $1 \mathrm{~L}$ TEGDME) and a porous catalyst cathode. The cell was sealed except for an $\mathrm{Al}$ grid window that exposed the porous catalyst cathode to $1 \mathrm{bar}$ $\mathrm{O}_{2}$ pressure in a glass chamber. The cell was installed into a Maccor cycler for the electrochemical measurements. The cell was maintained in 1 bar $\mathrm{O}_{2}$ atmosphere to avoid negative effects from humidity and $\mathrm{CO}_{2}$. The galvanostatic charge-discharge cycling was conducted at a constant current density of $0.1 \mathrm{~mA} / \mathrm{cm}^{2}$. For comparison, a blank carbon fiber electrode without any catalyst was also tested using the same cell configuration and test method described above.

\subsection{References}

$1 \mathrm{Fu}, \mathrm{F}$. et al. Synthesis of single crystalline hexagonal nanobricks of $\mathrm{LiNi}_{1 / 3} \mathrm{Co}_{1 / 3}$ $\mathrm{Mn}_{1 / 3} \mathrm{O}_{2}$ with high percentage of exposed $\{010\}$ active facets as high rate performance cathode material for lithium-ion battery. Journal of Materials Chemistry A 1, 3860-3864 (2013).

2 Wei, G. Z. et al. Crystal habit-tuned nanoplate material of $\mathrm{Li}\left[\mathrm{Li}_{1 / 3-2 \times} / 3 \mathrm{Ni}_{\mathrm{x}} \mathrm{Mn}_{2 / 3}\right.$ $\mathrm{x} / 3] \mathrm{O}_{2}$ for high-rate performance lithium-ion batteries. Advanced Materials 22, 4364-4367 (2010).

3 Wang, L. et al. Crystal orientation tuning of $\mathrm{LiFePO}_{4}$ nanoplates for high rate lithium battery cathode materials. Nano Letters 12, 5632-5636 (2012).

4 Xiao, X. et al. Facile shape control of $\mathrm{Co}_{3} \mathrm{O}_{4}$ and the effect of the crystal plane on electrochemical performance. Advanced Materials 24, 5762-5766 (2012).

5 Chan, C. K. et al. Fast, completely reversible Li insertion in vanadium pentoxide nanoribbons. Nano Letters 7, 490-495 (2007). 
6 Yao, W., Long, F. \& Shahbazian-Yassar, R. Localized mechanical stress induced ionic redistribution in a layered $\mathrm{LiCoO}_{2}$ cathode. ACS Applied Materials \& Interfaces 8, 29391-29399 (2016).

7 Zhao, Y., Peng, L., Liu, B. \& Yu, G. Single-crystalline $\mathrm{LiFePO}_{4}$ nanosheets for high-rate Li-ion batteries. Nano Letters 14, 2849-2853 (2014).

8 Yuan, Y. et al. The influence of large cations on the electrochemical properties of tunnel-structured metal oxides. Nature Communications 7, 13374 (2016).

9 Jiang, H., Zhao, T., Yan, C., Ma, J. \& Li, C. Hydrothermal synthesis of novel $\mathrm{Mn}_{3}$ $\mathrm{O}_{4}$ nano-octahedrons with enhanced supercapacitors performances. Nanoscale 2 , 2195-2198 (2010).

$10 \mathrm{Su}, \mathrm{D}$., Dou, S. \& Wang, G. Single crystalline $\mathrm{Co}_{3} \mathrm{O}_{4}$ nanocrystals exposed with different crystal planes for $\mathrm{Li}_{2} \mathrm{O}_{2}$ batteries. Scientific Reports 4, 5767 (2014).

11 Kitchaev, D. A., Dacek, S. T., Sun, W. \& Ceder, G. Thermodynamics of phase selection in $\mathrm{MnO}_{2}$ framework structures through alkali intercalation and hydration. Journal of the American Chemical Society 139, 2672-2681 (2017).

12 Ghodbane, O., Pascal, J.-L. \& Favier, F. Microstructural effects on charge-storage properties in $\mathrm{MnO}_{2}$-based electrochemical supercapacitors. ACS Applied Materials \& Interfaces 1, 1130-1139 (2009).

13 Jiang, H., Zhao, T., Ma, J., Yan, C. \& Li, C. Ultrafine manganese dioxide nanowire network for high-performance supercapacitors. Chemical Communications 47, 1264-1266 (2011).

14 Débart, A., Paterson, A. J., Bao, J. \& Bruce, P. G. $\alpha-\mathrm{MnO}_{2}$ nanowires: A catalyst for the $\mathrm{O}_{2}$ electrode in rechargeable lithium batteries. Angewandte Chemie International Edition 120, 4597-4600 (2008).

15 Truong, T. T., Liu, Y., Ren, Y., Trahey, L. \& Sun, Y. Morphological and crystalline evolution of nanostructured $\mathrm{MnO}_{2}$ and its application in lithium-air batteries. ACS Nano 6, 8067-8077 (2012).

16 Thapa, A. K., Hidaka, Y., Hagiwara, H., Ida, S. \& Ishihara, T. Mesoporous $\beta$ $\mathrm{MnO}_{2}$ air electrode modified with Pd for rechargeability in lithium-Air battery. Journal of the Electrochemical Society 158, A1483-A1489 (2011).

17 Ida, S. et al. Manganese oxide with a card-house-like structure reassembled from nanosheets for rechargeable Li-air battery. Journal of Power Sources 203, 159164 (2012). 
18 Song, K. et al. $\alpha-\mathrm{MnO}_{2}$ nanowire catalysts with ultra-high capacity and extremely low overpotential in lithium-air batteries through tailored surface arrangement. Physical Chemistry Chemical Physics 15, 20075-20079 (2013).

19 Cao, Y. et al. $\alpha-\mathrm{MnO}_{2}$ nanorods grown in situ on graphene as catalysts for $\mathrm{Li}-\mathrm{O}_{2}$ batteries with excellent electrochemical performance. Energy \& Environmental Science 5, 9765-9768 (2012).

20 Yang, Y., Shi, M., Li, Y.-S. \& Fu, Z.-W. $\mathrm{MnO}_{2}$-graphene composite air electrode for rechargeable Li-air batteries. Journal of the Electrochemical Society 159, A1917-A1921 (2012).

21 Li, J., Wang, N., Zhao, Y., Ding, Y. \& Guan, L. $\mathrm{MnO}_{2}$ nanoflakes coated on multi-walled carbon nanotubes for rechargeable lithium-air batteries.

Electrochemistry Communications 13, 698-700 (2011).

22 Zhang, G. et al. $\alpha-\mathrm{MnO}_{2} /$ carbon nanotube/carbon nanofiber composite catalytic air electrodes for rechargeable lithium-air batteries. Journal of The Electrochemical Society 158, A822-A827 (2011).

$23 \mathrm{Hu}, \mathrm{X}$., Han, X., Hu, Y., Cheng, F. \& Chen, J. $\varepsilon-\mathrm{MnO}_{2}$ nanostructures directly grown on Ni foam: a cathode catalyst for rechargeable $\mathrm{Li}-\mathrm{O}_{2}$ batteries. Nanoscale 6, 3522-3525 (2014).

24 Thapa, A. K., Saimen, K. \& Ishihara, T. $\mathrm{Pd} / \mathrm{MnO}_{2}$ air electrode catalyst for rechargeable lithium/air battery. Electrochemical and Solid-State Letters 13, A165-A167 (2010).

25 Thapa, A. K. \& Ishihara, T. Mesoporous $\alpha-\mathrm{MnO}_{2} / \mathrm{Pd}$ catalyst air electrode for rechargeable lithium-air battery. Journal of Power Sources 196, 7016-7020 (2011).

26 Liu, J., Younesi, R., Gustafsson, T., Edström, K. \& Zhu, J. Pt/ $\alpha-\mathrm{MnO}_{2}$ nanotube: A highly active electrocatalyst for $\mathrm{Li}_{-} \mathrm{O}_{2}$ battery. Nano Energy 10, 19-27 (2014).

27 Jin, L. et al. Titanium containing $\gamma-\mathrm{MnO}_{2}$ (TM) hollow spheres: One-step synthesis and catalytic activities in Li/air batteries and oxidative chemical reactions. Advanced Functional Materials 20, 3373-3382 (2010).

28 Tompsett, D. A., Parker, S. C. \& Islam, M. S. Rutile ( $\beta$-) $\mathrm{MnO}_{2}$ surfaces and vacancy formation for high electrochemical and catalytic performance. Journal of the American Chemical Society 136, 1418-1426 (2014).

29 Dawson, J. A., Chen, H. \& Tanaka, I. First-principles calculations of oxygen vacancy formation and metallic behavior at a $\beta-\mathrm{MnO}_{2}$ grain boundary. $A C S$ Applied Materials \& Interfaces 7, 1726-1734 (2015). 
30 Li, L. et al. Insight into the effect of oxygen vacancy concentration on the catalytic performance of $\mathrm{MnO}_{2}$. ACS Catalysis 5, 4825-4832 (2015).

31 Mellan, T. A. et al. Lithium and oxygen adsorption at the $\beta-\mathrm{MnO}_{2}(110)$ surface. Journal of Materials Chemistry A 1, 14879-14887 (2013).

32 Yao, W. et al. Energy-driven surface evolution in beta- $\mathrm{MnO}_{2}$ structures. Nano Research 11, 206-215 (2018).

33 Yao, W. et al. Cations controlled growth of $\beta-\mathrm{MnO}_{2}$ crystals with tunable facets for electrochemical energy storage. Nano Energy 48, 301-311 (2018).

34 Yuan, Y. et al. Atomistic insights into the oriented attachment of tunnel-based oxide nanostructures. ACS Nano 10, 539-548 (2016).

35 Devaraj, S. \& Munichandraiah, N. Effect of crystallographic structure of $\mathrm{MnO}_{2}$ on its electrochemical capacitance properties. The Journal of Physical Chemistry C 112, 4406-4417 (2008).

36 Sun, C., Zhang, Y., Song, S. \& Xue, D. Tunnel-dependent supercapacitance of $\mathrm{MnO}_{2}$ : effects of crystal structure. Journal of Applied Crystallography 46, 1128 1135 (2013).

37 Jung, H.-G., Hassoun, J., Park, J.-B., Sun, Y.-K. \& Scrosati, B. An improved high-performance lithium-air battery. Nature Chemistry 4, 579 (2012).

38 Adams, B. D. et al. Current density dependence of peroxide formation in the Li$\mathrm{O}_{2}$ battery and its effect on charge. Energy \& Environmental Science 6, 17721778 (2013).

39 Johnson, L. et al. The role of $\mathrm{LiO}_{2}$ solubility in $\mathrm{O}_{2}$ reduction in aprotic solvents and its consequences for $\mathrm{Li}_{2} \mathrm{O}_{2}$ batteries. Nature Chemistry 6, 1091-1099 (2014).

40 Aetukuri, N. B. et al. Solvating additives drive solution-mediated electrochemistry and enhance toroid growth in non-aqueous $\mathrm{Li}-\mathrm{O}_{2}$ batteries. Nature Chemistry 7, 50 (2014).

41 Kwabi, D. G. et al. Experimental and computational analysis of the solventdependent $\mathrm{O}_{2} / \mathrm{Li}^{+}-\mathrm{O}_{2}{ }^{-}$redox couple: Standard potentials, coupling strength, and implications for lithium-oxygen batteries. Angewandte Chemie International Edition 55, 3129-3134 (2016).

42 Abraham, K. M. Electrolyte-directed reactions of the oxygen electrode in lithiumair batteries. Journal of The Electrochemical Society 162, A3021-A3031 (2015). 
43 Hummelshøj, J. S. et al. Communications: Elementary oxygen electrode reactions in the aprotic Li-air battery. The Journal of Chemical Physics 132, 071101 (2010).

44 Aurbach, D., McCloskey, B. D., Nazar, L. F. \& Bruce, P. G. Advances in understanding mechanisms underpinning lithium-air batteries. Nature Energy 1, 16128 (2016). 


\section{Chapter 7 Conclusion}

Layered $\mathrm{LiCoO}_{2}$ and tunnel based $\beta-\mathrm{MnO}_{2}$ crystals were selected to study the interplay of lithium ion transportation and crystal facets of the cathode crystals. Four detailed projects were explored, including (1) the effect of localized mechanical stresses on the lithium ion diffusion studied by C-AFM; (2) the lateral surface evolution mechanism of $\beta-\mathrm{MnO}_{2}$ nanowire and microrod; (3) facet-controlled synthesis of $\beta-\mathrm{MnO}_{2}$ crystals with tunable morphologies and its application in lithium ion batteries; (4) the effect of crystal facets on the performance of $\beta-\mathrm{MnO}_{2}$ crystals in supercapacitors and lithium-air batteries. The main conclusions are summarized as follows.

(1) The localized mechanical stress generated from the AFM tip can induce reversible resistive switching (lithium ion redistribution) under concentrated electric field. Higher mechanical compression forces improved the current responses from the layered $\mathrm{LiCoO}_{2}$ substrate, which was related to the deeper penetration of the electric field. The effect of tip-sample contact area on the current response was thoroughly investigate based on a comparative study on a conductive HOPG substrate, which further demonstrate the improve current response was mainly related to lithium ion redistribution.

(2) $\beta-\mathrm{MnO}_{2}$ nanowire and microrod showed similar lateral surface evolution process with the facet evolution from $\{100\}$ surfaces to more stable $\{110\}$ surfaces. The surface evolution was realized following two ways: one is self-growth along the active $\{100\}$ surfaces; another is oriented attachment between different crystals 
along the unstable $\{100\}$ surfaces. High density defects were generated during these two processes.

(3) The growth mechanism of $\beta-\mathrm{MnO}_{2}$ bipyramid prism structure was found to follow oriented attachment of $\alpha-\mathrm{K}_{0.5} \mathrm{Mn}_{4} \mathrm{O}_{8}$ nanowires and subsequently direct phase

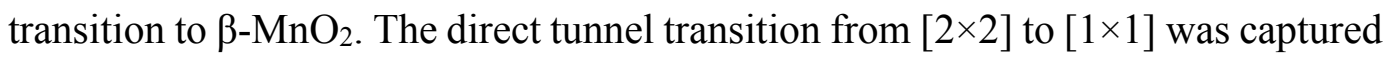
based on cross-sectional analysis and STEM imaging. The role of $\mathrm{K}^{+}$cations during the morphology evolution was proposed to be related with the formation of $\alpha$ $\mathrm{K}_{0.5} \mathrm{Mn}_{4} \mathrm{O}_{8}$ intermediate and the subsequent phase transition. High concentration of external $\mathrm{K}^{+}$cations impeded the extraction of tunnel cations during the phase transition, which reduced the dimension of $\beta-\mathrm{MnO}_{2}$ crystal along the tunnel direction. This reduced dimension along tunnel direction facilitated lithium ion diffusion and offered better rate performance than traditional nanorod morphologies.

(4) The $\beta-\mathrm{MnO}_{2}$ bipyramid prism and octahedron structure were identified to have high occupancy $(\sim 80 \%)$ of $\{100\}$ and $\{111\}$ facets, respectively. Applying these two morphologies as the electrode for supercapacitors showed the $\{100\}$ facets of $\beta$ $\mathrm{MnO}_{2}$ can deliver higher specific capacitance than the $\{111\}$ facets due to a relatively higher exposed Mn centers. As the cathode catalyst for lithium-air batteries, both $\{100\}$ and $\{111\}$ facets showed catalytic activities in reducing the charge-discharge overpotential compared with bare carbon cathode. The $\{100\}$ facets exhibited slightly better performance in reducing the charging overpotential. Further study of the discharge product showed that the $\{111\}$ and $\{100\}$ facets facilitated the formation of $\mathrm{Li}_{2} \mathrm{O}_{2}$ with toroids and thin film shapes, respectively. 
The different formation mechanism of $\mathrm{Li}_{2} \mathrm{O}_{2}$ was related to the different surface adsorption energies of $\mathrm{LiO}_{2}$ intermediate on the $\{111\}$ and $\{100\}$ facets of $\beta-\mathrm{MnO}_{2}$. 


\section{Chapter 8 Future Work}

In terms of the growth mechanism study of $\beta-\mathrm{MnO}_{2}$, the completed projects have demonstrated the possibility of using tunnel cations to tune the morphology of $\mathrm{MnO}_{2}$ crystals, which could be possibly used for the facet engineering of other tunneled crystals as well, such as todorokite $\mathrm{MnO}_{2}$. In terms of the application in rechargeable batteries, $\beta$ $\mathrm{MnO}_{2}$ nanowire has found applications as the cathode for sodium ion batteries. However, whether $\mathrm{Na}^{+}$ions can intercalate into the $[1 \times 1]$ tunnel of $\beta-\mathrm{MnO}_{2}$ or not still lack direct evidences. Moreover, various $\mathrm{MnO}_{2}$ crystals have found applications as the cathode for magnesium-ion batteries with different electrochemical performances. Comparative studies are needed to identify the preferred phase as the high-performance cathode for $\mathrm{Mg}^{2+}$ (de)intercalation. In terms of the application in supercapacitors, the specific capacitance obtained from various $\mathrm{MnO}_{2}$ crystals is still far below the theoretical capacitance of 1370 $\mathrm{F} \cdot \mathrm{g}^{-1}$. Although lots of methods have been tried (including the introduction of conductive substrates, atomic doping, etc.), there is still room for further improvement of the electrochemical performances.

\subsection{Effect of $\mathrm{Mg}^{2+}$ cations on the formation of todorokite $\mathrm{MnO}_{2}$}

The synthesis of todorokite $\mathrm{MnO}_{2}$ generally follows three steps: (1) the preparation of Nabirnessite precursor; (2) the formation of Mg-buserite through an ion-exchange method; (3) hydrothermal treatment of Mg-buserite to form Mg-OMS-1. The hydrothermal treatment of Mg-buserite usually take place in $1 \mathrm{M} \mathrm{MgCl}_{2}$ solution. Most of the XRD patterns of the obtained structure showed sharp peaks of (002) plane near $2 \theta=9.3^{\circ}$, indicating a planar distance around $9.5 \AA$. However, this couldn't support the conclusion 
that $[3 \times 3]$ tunnels were obtained in the structure. Our recent work observed large amount of $[3 \times 4],[3 \times 5],[3 \times 7]$ tunnels in the so-called todorokite structure instead of pure $[3 \times 3]$ tunnels. It is proposed that the concentration of $\mathrm{Mg}^{2+}$ ions during ion exchange and subsequently hydrothermal synthesis greatly affect the tunnel dimensions. Our assumptions are as follows:

(1) The concentration of $\mathrm{Mg}^{2+}$ ions during ion exchange determines the amount of $\mathrm{Mn}^{3+}$ obtained in the layered Mg-buserite.

(2) The $\mathrm{Mn}^{3+}$ centers act as the nucleation locations for the layer-to-tunnel transitions. Higher concentration of $\mathrm{Mg}^{2+}$ exchange ions will induce smaller tunnels, such as [3×3], [3×2], which plays a key role in controlling the tunnel size of the todorokite crystals.

(3) The concentration of $\mathrm{Mg}^{2+}$ ions during hydrothermal synthesis serves as the stabilizing ions in the tunnel during the phase transition. Increase this concentration would increase the crystallinity of the obtained todorokite crystals.

Tian et al studied the effect of $\mathrm{Mg}^{2+}$ concentration during ion exchange for the synthesis of Mg-OMS-1. The concentration of $\mathrm{MgCl}_{2}$ for hydrothermal synthesis, however, was kept at $1 \mathrm{M}$. It was found that the staring concentration of $\mathrm{MgCl}_{2}$ within $0.04 \mathrm{M}$ to $0.09 \mathrm{M}$ could facilitate the formation of Mg-buserite with clear 9.6 $\AA$ XRD peaks. Following the hydrothermal synthesis, sharp peaks were clearly observed at $2 \theta=9.3^{\circ}$, corresponding to the $9.6 \AA$ spacing of the $[3 \times \mathrm{n}]$ tunnel. ${ }^{1}$ The correct range of $\mathrm{MgCl}_{2}$ concentration for starting material was found to lie between 0.34 and 0.42 for the ratio of $\mathrm{Mg} / \mathrm{Mn}$. 
However, the study of $\mathrm{Mg}^{2+}$ ion effect only considered the concentration of $\mathrm{Mg}^{2+}$ ions in the precursor. The concentration of $\mathrm{Mg}^{2+}$ ions during hydrothermal synthesis was ignored. It was observed that the sample 4 with $0.2 \mathrm{M} \mathrm{MgCl}_{2}$ starting mixture formed clear $[3 \times \mathrm{n}]$ tunnel although the $9.6 \AA$ peak was not observed previously after ion exchange. This could be due to the $1 \mathrm{M} \mathrm{MgCl}_{2}$ used during hydrothermal synthesis.

Another point needs clarification is the tunnels formed within the todorokite structure. Although the XRD pattern shows peaks of the $9.6 \AA$ spacing, it couldn't be solely assigned to pure [3×3] tunnels. As long as one dimension of the tunnels is $9.6 \AA$, a mixture of $[3 \times n]$ tunnels could also give similar peaks near the $9.6 \AA$ spacing. A more detailed examination of the tunnels (atomic scale imaging, plus cross-section analysis) would be needed, and low-angle XRD data would be also helpful for the identification of possible larger dimensions.

\subsection{Whether $\mathrm{Na}^{+}$ions can intercalate into $[1 \times 1]$ tunnel of $\beta-\mathrm{MnO}_{2}$}

The application of $\beta-\mathrm{MnO}_{2}$ as the cathode for $\mathrm{Na}$-ion batteries was reported recently by $\mathrm{Su}$ et al..$^{2,3}$ A high initial capacity of $298 \mathrm{mAh} / \mathrm{g}$ was obtained using $\beta-\mathrm{MnO}_{2}$ nanorods the cathode, even higher than that of the $\alpha-\mathrm{MnO}_{2}$ counterpart $(278 \mathrm{mAh} / \mathrm{g}) .^{3}$ The excellent performance was ascribed to more compact tunnels in $\beta-\mathrm{MnO}_{2}$ than $\alpha-\mathrm{MnO}_{2}$. The author claimed that $\mathrm{Na}^{+}$ions can intercalate into the $[1 \times 1]$ tunnel of $\beta-\mathrm{MnO}_{2}$ simply by comparing

the ion radius $(1.02 \AA$ vs. $2.3 \AA)$. To demonstrate whether $[1 \times 1]$ tunnels can sustain $\mathrm{Na}^{+}$ion intercalation, we adopted in situ Nanofactory TEM holder to check the sodium ion insertion behavior of $\beta-\mathrm{MnO}_{2}$ nanorods synthesized following the same route. Our preliminary 
showed the $\beta-\mathrm{MnO}_{2}$ nanorods underwent direct phase transition after touching the sodium source. Further ex-situ battery test showed the $\beta-\mathrm{MnO}_{2}$ nanorods did not have the same (de)intercalation voltage plateau as observed in lithium systems. Recently, $\beta-\mathrm{NaMnO}_{2}$ was reported as a high-capacity cathode for sodium ion batteries. The $\beta-\mathrm{NaMnO}_{2}$ cathode exhibited $190 \mathrm{mAh} / \mathrm{g}$ initial capacity at $\mathrm{C} / 20 .{ }^{4}$ The XRD pattern of $\beta-\mathrm{NaMnO}_{2}$ crystal is similar with that reported in Su et al's work.

It should be safe to propose that the $[1 \times 1]$ tunnel of $\beta-\mathrm{MnO}_{2}$ is inaccessible for $\mathrm{Na}^{+}$ ions intercalation. Previously observed high-performance of $\beta-\mathrm{MnO}_{2}$ by Su et al is mostly related to a deep cycling (voltage window reached 1.0V) and unclear conversion reactions. Further STEM imaging of the tunnel structure before and after cycling would be a good evidence to support the assumption.

\subsection{Effect of crystal phases on the performance of $\mathrm{MnO}_{2}$ as the cathode for Mg-ion batteries}

Compared with lithium anode rechargeable batteries, Mg-ion batteries can provide much higher volumetric energy density $\left(\sim 3833 \mathrm{mAh} / \mathrm{cm}^{3}\right.$ vs. $\left.\sim 2046 \mathrm{mAh} / \mathrm{cm}^{3}\right)$. Despite the earthabundancy of Mg element, Mg metal anode does not suffer from dendrite growth as lithium and sodium metal anode. However, Mg-ion systems still lack stable cathodes and proper electrolytes for practical applications. Several $\mathrm{MnO}_{2}$ crystals have been investigated as the cathode for $\mathrm{Mg}$-ion batteries, including $\alpha-\mathrm{MnO}_{2}, \mathrm{MnO}_{2}$ todorokite, $\mathrm{MnO}_{2}$ birnessite, spinel $\mathrm{MnO}_{2}$, and post-spinel $\mathrm{MnO}_{2}$. 
Zhang et al adopted $\alpha-\mathrm{MnO}_{2}$ as the cathode for $\mathrm{Mg}^{2+}$ intercalation and obtained 240 $\mathrm{mAh} / \mathrm{g}(0.015 \mathrm{C})$ at the first cycle using Mg metal as the anode; the voltage range used was $0.8 \sim 3.0 \mathrm{~V}$ vs. $\mathrm{Mg} / \mathrm{Mg}^{2+}{ }^{2}$. Similar to lithium-ion batteries, introducing conductive composite can further improve the performance of $\alpha-\mathrm{MnO}_{2}$ cathode. Rasul et al used $\alpha$ $\mathrm{MnO}_{2} /$ acetylene black composite as the cathode and obtained $475 \mathrm{mAh} / \mathrm{g}$ first-cycle capacity. ${ }^{6,7}$ However, severe capacity loss was observed after a few cycles, which was attributed to either the instability of the [2×2] tunnel upon $\mathrm{Mg}^{2+}$ ions intercalation or the Mn dissolution in the electrolyte. It was also found the particle size of $\alpha-\mathrm{MnO}_{2}$ greatly affected the discharge capacity; smaller particles gave higher initial capacities.

Todorokite $\mathrm{MnO}_{2}$ composed of large [3×3] tunnels $(6.9 \AA \times 6.9 \AA)$, which could be used for the intercalation of $\mathrm{Mg}^{2+}$ ions. Kumagai et al obtained $85 \mathrm{mAh} / \mathrm{g}$ first-cycle capacity for todorokite $\mathrm{MnO}_{2}{ }^{8}$ However, the cycling performance was not provided and the structure of the todorokite $\mathrm{MnO}_{2}$ may not be exactly [3×3] tunneled. Zhang et al obtained $300 \mathrm{mAh} / \mathrm{g}$ first-cycle capacity using a $\mathrm{MgCl}_{2}$ aqueous electrolyte and obtained 300 cycles with $83.7 \%$ capacity retention. ${ }^{9}$ However, whether it was $\mathrm{Mg}^{2+}$ or proton intercalation still need further investigation.

Birnessite $\delta-\mathrm{MnO}_{2}$ with different stabilizing ions were extensively examined as the cathode for Mg-ion intercalation. Rasul et al used Birnessite/acetylene black composite as the cathode and obtained $109 \mathrm{mAh} / \mathrm{g}$ first-cycle capacity. It was also found that the Birnessite with $\mathrm{Mg}^{2+}$ stabilizing ions had the best performances. ${ }^{7}$ The capacity retention of Birnessite is better than $\alpha-\mathrm{MnO}_{2}$. Nam et al achieved $231.1 \mathrm{mAh} / \mathrm{g}$ with over 10000 cycle life using Birnessite $\mathrm{MnO}_{2}$ as the cathode with the introduction of crystal water in the 
layered structure of birnessite. The crystal water was believed to facilitate the $\mathrm{Mg}^{2+}$ ions (de)intercalation. ${ }^{10}$ Sun et al further investigated the effect of aqueous and non-aqueous electrolyte on the mechanism of $\mathrm{Mg}^{2+}$ intercalation in layered $\mathrm{MnO}_{2}$. A conversion mechanism was observed when cycling birnessite in non-aqueous electrolyte; while intercalation and particle phase transition to a spinel phase were observed when cycling in aqueous electrolyte. ${ }^{11}$ Although long-cycling capability was observed in the birnessite $\mathrm{MnO}_{2}$ in aqueous electrolyte, the intercalation of $\mathrm{Mg}^{2+}$ still lack direct evidence and further studies are needed to clarify whether it was $\mathrm{Mg}^{2+}$ ion or proton intercalation.

Spinel $\lambda-\mathrm{MnO}_{2}$ was also investigated as the cathode for $\mathrm{Mg}$ ion intercalation. With a $\mathrm{MgCl}_{2}$ aqueous electrolyte, a high first-cycle capacity of $545.6 \mathrm{mAh} / \mathrm{g}$ was achieved. ${ }^{12}$ However, the theoretical capacity of $\lambda-\mathrm{MnO}_{2}$ is around $308 \mathrm{mAh} / \mathrm{g}$. This may due to the improper selection of the voltage window which causes proton cycling. ${ }^{13}$ Using atomic scale STEM, Kim et al directly captured the intercalated Mg ions in the spinel structure of $\lambda-\mathrm{MnO}_{2} \cdot{ }^{14}$

In summary, there are still lot of issues and challenges for $\mathrm{MnO}_{2}$ to be used as a practical $\mathrm{Mg}^{2+}$ ion battery cathode. The key question so far for $\mathrm{MnO}_{2}$ would be the role of $\mathrm{H}_{2} \mathrm{O}$ during $\mathrm{Mg}^{2+}$ intercalation with the aqueous electrolyte. Atomic-scale STEM imaging in combination with other in situ spectroscopy techniques would be the strong tools to clarify the current observations and provide important guidance for further design and fabrication of the $\mathrm{MnO}_{2}$ cathode. 


\subsection{References}

1 Tian, Z.-R., Yin, Y.-G., Suib, S. L. \& O'Young, C. Effect of $\mathrm{Mg}^{2+}$ ions on the formation of todorokite type manganese oxide octahedral molecular sieves. Chemistry of Materials 9, 1126-1133 (1997).

$2 \mathrm{Su}, \mathrm{D} ., \mathrm{Ahn}, \mathrm{H} .-\mathrm{J}$. \& Wang, G. $\beta-\mathrm{MnO}_{2}$ nanorods with exposed tunnel structures as high-performance cathode materials for sodium-ion batteries. NPG Asia Materials 5, e70 (2013).

$3 \mathrm{Su}, \mathrm{D} ., \mathrm{Ahn}, \mathrm{H} .-\mathrm{J} . \&$ Wang, G. Hydrothermal synthesis of $\alpha-\mathrm{MnO}_{2}$ and $\beta-\mathrm{MnO}_{2}$ nanorods as high capacity cathode materials for sodium ion batteries. Journal of Materials Chemistry A 1, 4845-4850 (2013).

4 Billaud, J. et al. $\beta-\mathrm{NaMnO}_{2}$ : a high-performance cathode for sodium-ion batteries. Journal of the American Chemical Society 136, 17243-17248 (2014).

5 Zhang, R. et al. $\alpha-\mathrm{MnO}_{2}$ as a cathode material for rechargeable $\mathrm{Mg}$ batteries. Electrochemistry Communications 23, 110-113 (2012).

6 Rasul, S., Suzuki, S., Yamaguchi, S. \& Miyayama, M. Synthesis and electrochemical behavior of hollandite $\mathrm{MnO}_{2}$ /acetylene black composite cathode for secondary Mg-ion batteries. Solid State Ionics 225, 542-546 (2012).

7 Rasul, S., Suzuki, S., Yamaguchi, S. \& Miyayama, M. High capacity positive electrodes for secondary Mg-ion batteries. Electrochimica Acta 82, 243-249 (2012).

8 Kumagai, N., Komaba, S., Sakai, H. \& Kumagai, N. Preparation of todorokitetype manganese-based oxide and its application as lithium and magnesium rechargeable battery cathode. Journal of Power Sources 97, 515-517 (2001).

9 Zhang, H. et al. Octahedral magnesium manganese oxide molecular sieves as the cathode material of aqueous rechargeable magnesium-ion battery. Electrochimica Acta 229, 371-379 (2017).

10 Nam, K. W. et al. The high performance of crystal water containing manganese birnessite cathodes for magnesium batteries. Nano Letters 15, 4071-4079 (2015).

11 Sun, X., Duffort, V., Mehdi, B. L., Browning, N. D. \& Nazar, L. F. Investigation of the mechanism of $\mathrm{Mg}$ insertion in birnessite in nonaqueous and aqueous rechargeable Mg-ion batteries. Chemistry of Materials 28, 534-542 (2016).

12 Yuan, C. et al. Investigation of the intercalation of polyvalent cations $\left(\mathrm{Mg}^{2+}\right.$, $\mathrm{Zn}^{2+}$ ) into $\lambda-\mathrm{MnO}_{2}$ for rechargeable aqueous battery. Electrochimica Acta 116, 404-412 (2014). 
13 Canepa, P. et al. Odyssey of multivalent cathode materials: Open questions and future challenges. Chemical Reviews 117, 4287-4341 (2017).

14 Kim, C. et al. Direct observation of reversible magnesium ion intercalation into a spinel oxide host. Advanced Materials 27, 3377-3384 (2015). 


\section{Appendix A. Supporting information for Chapter 3}

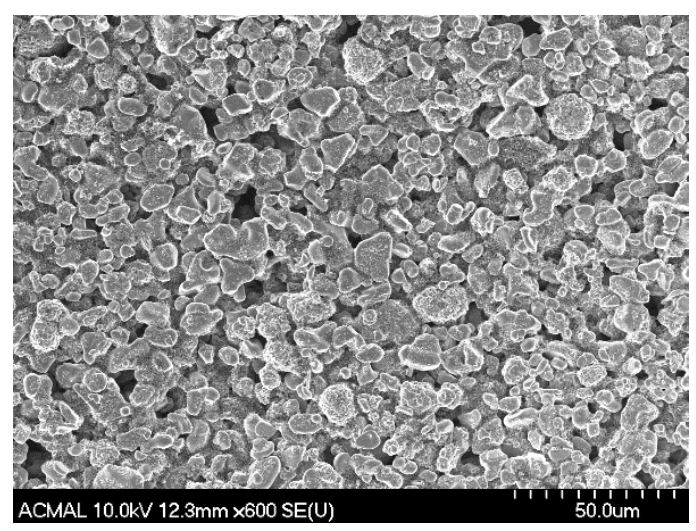

Figure S3-1. SEM image of the polycrystalline $\mathrm{LiCoO}_{2}$ thin film.

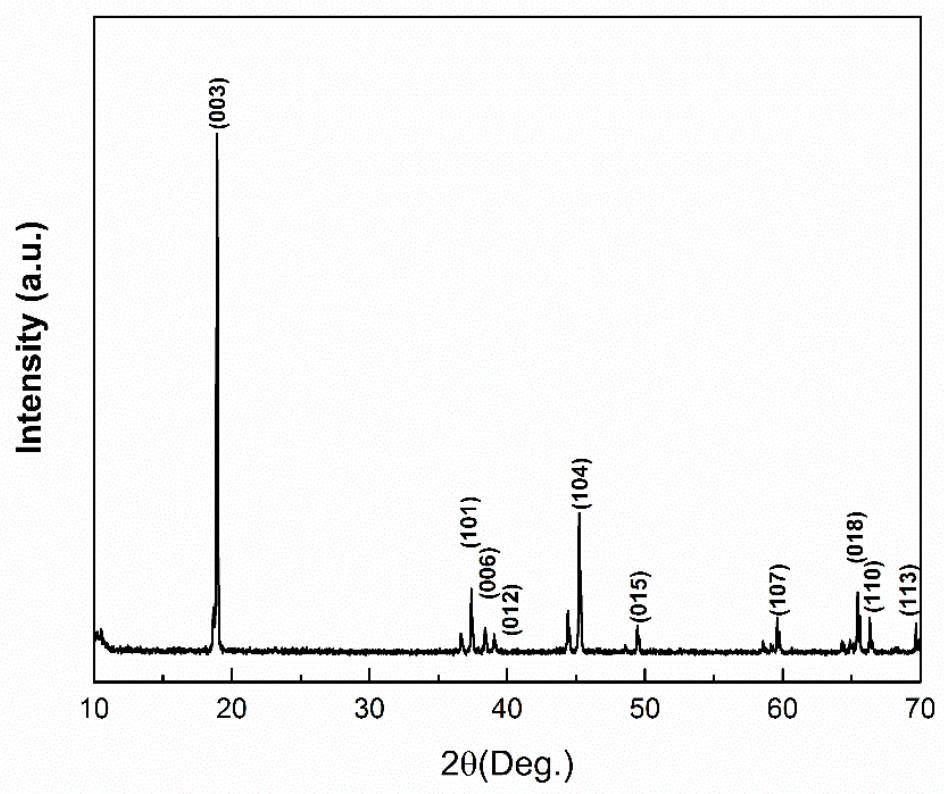

Figure S3-2. XRD pattern of the polycrystalline $\mathrm{LiCoO}_{2}$ thin film. Nonindexed peaks at $37.4^{\circ}$ and $44.4^{\circ}$ correspond to $\mathrm{Co}_{3} \mathrm{O}_{4}$ phase (JCPDS 801544), which shall come from the manufacturing process. 

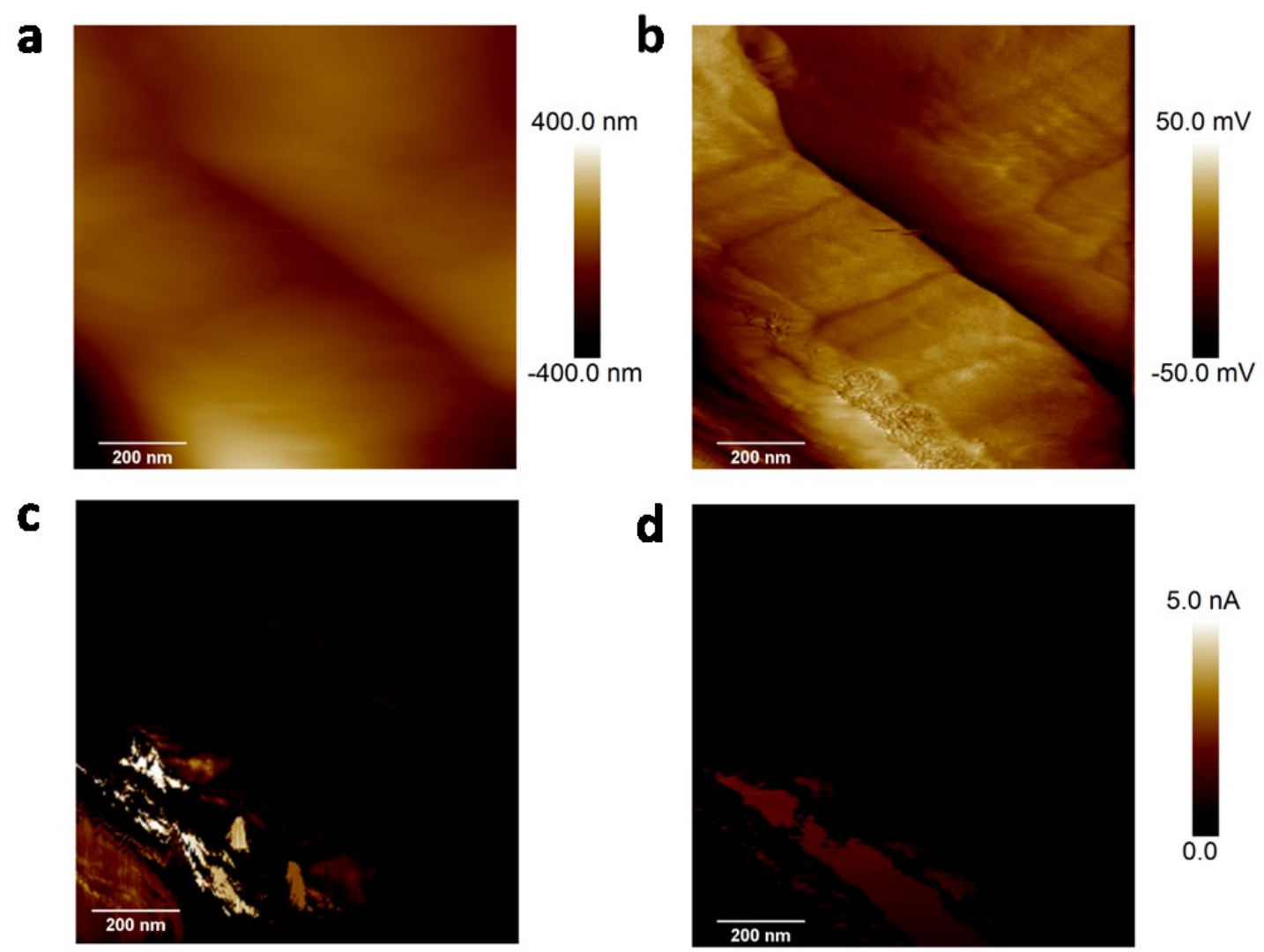

Figure S3-3. Height (a) and deflection (b) images from the $\mathrm{LiCoO}_{2}$ thin film substrate. Electrical current mapping under a DC biased voltage of $2 \mathrm{~V}$ at scan direction of 0 degree (c) and 90 degree (d). Figure S3d was rotated back 90 degree for better comparison with Figure S3c. 
a

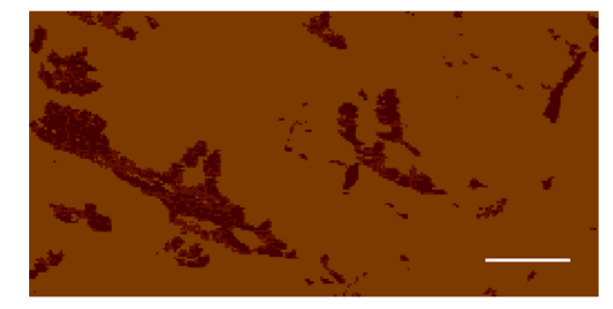

C

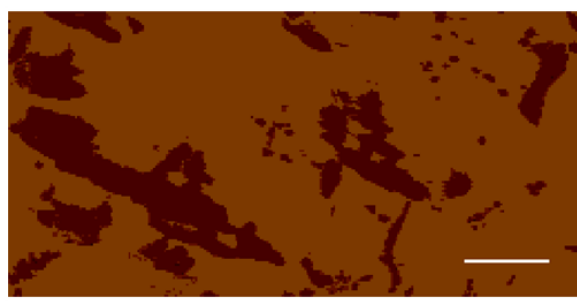

$131.6 \pm 5.4 \mathrm{nN}$ b

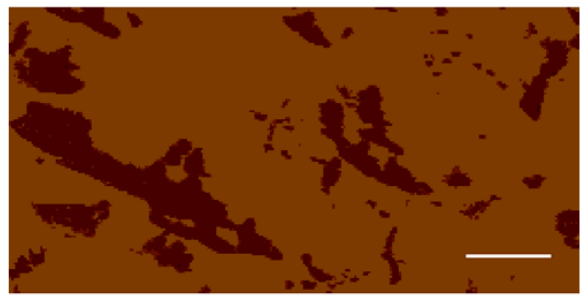

$78.9 \pm 3.2 \mathrm{nN}$

d

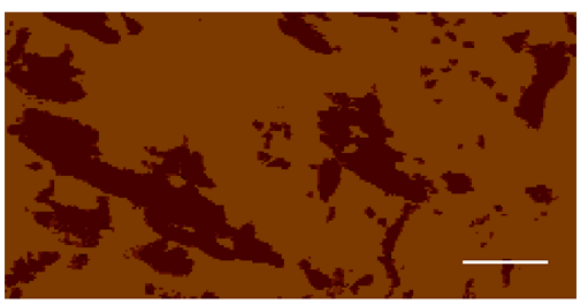

$184.2 \pm 7.5 \mathrm{nN}$
200.0 pA

-200.0 pA

Figure S3-4. Electric current mapping of the corresponding area in the layered $\mathrm{LiCoO}_{2}$ substrate during the stress loading process without a bias voltage. Scale bars are $300 \mathrm{~nm}$.

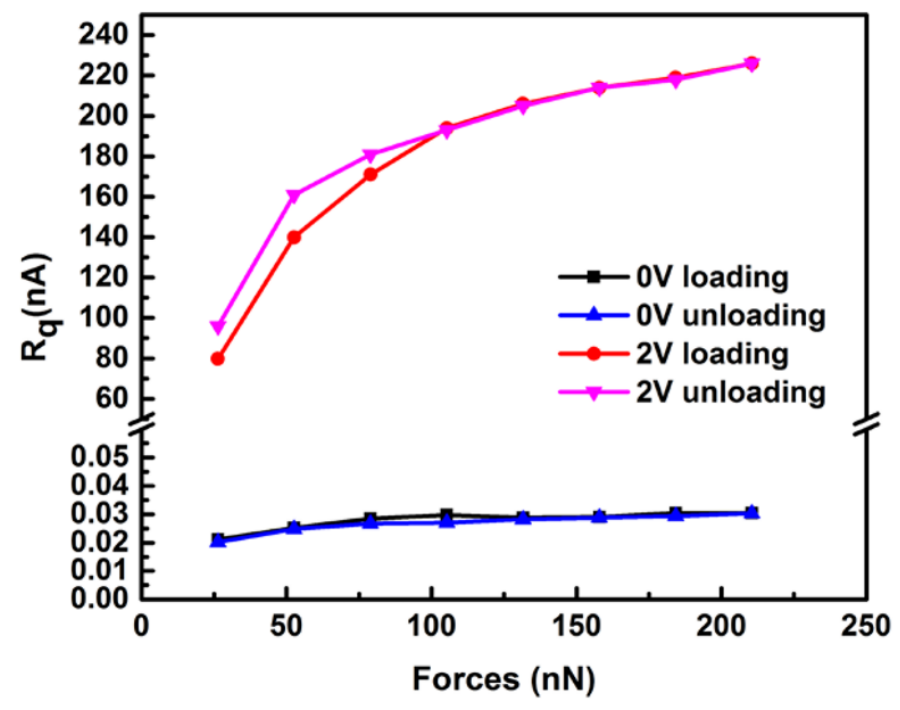

Figure S3-5. Root mean square roughness $\left(\mathrm{R}_{\mathrm{q}}\right)$ of electrical current from $\mathrm{LiCoO}_{2}$ thin film as a function of applied forces under a DC voltage of $0 \mathrm{~V}$ and $2 \mathrm{~V}$. 


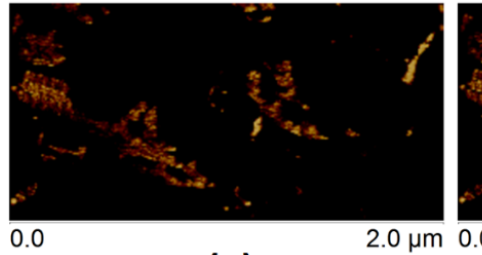

(a)

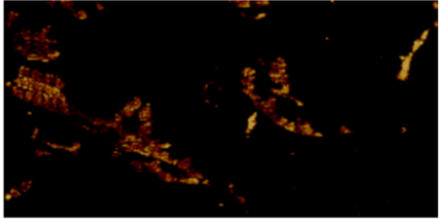

(b)

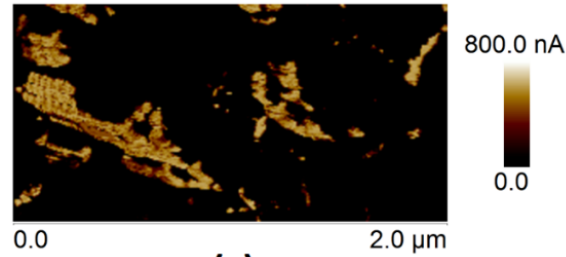

(c)

Figure S3-6. Electrical current images obtained under an applied force of $26.3 \mathrm{nN}$ at $0 \mathrm{~min}$ (a) and around $5 \mathrm{~min}$ (b). And current images obtained at around 10 min with an applied force of $52.6 \mathrm{nN}$. The applied DC bias voltage was $2 \mathrm{~V}$.

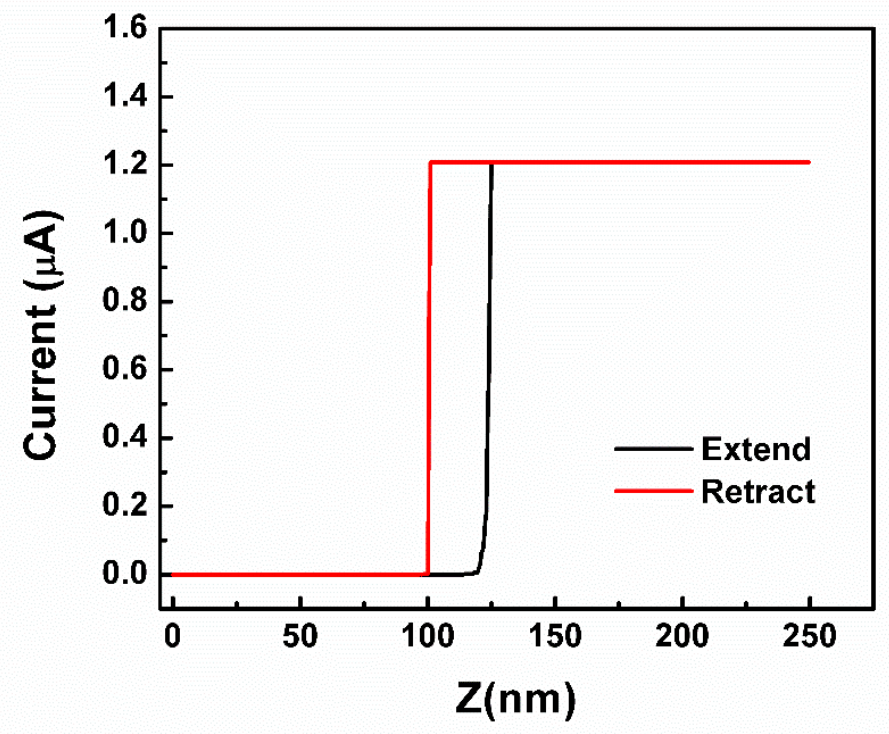

Figure S3-7. Ramp the tip-sample distance Z (distance between the substrate and the cantilever rest position) versus current output at spot 3 (Figure 3 ). 

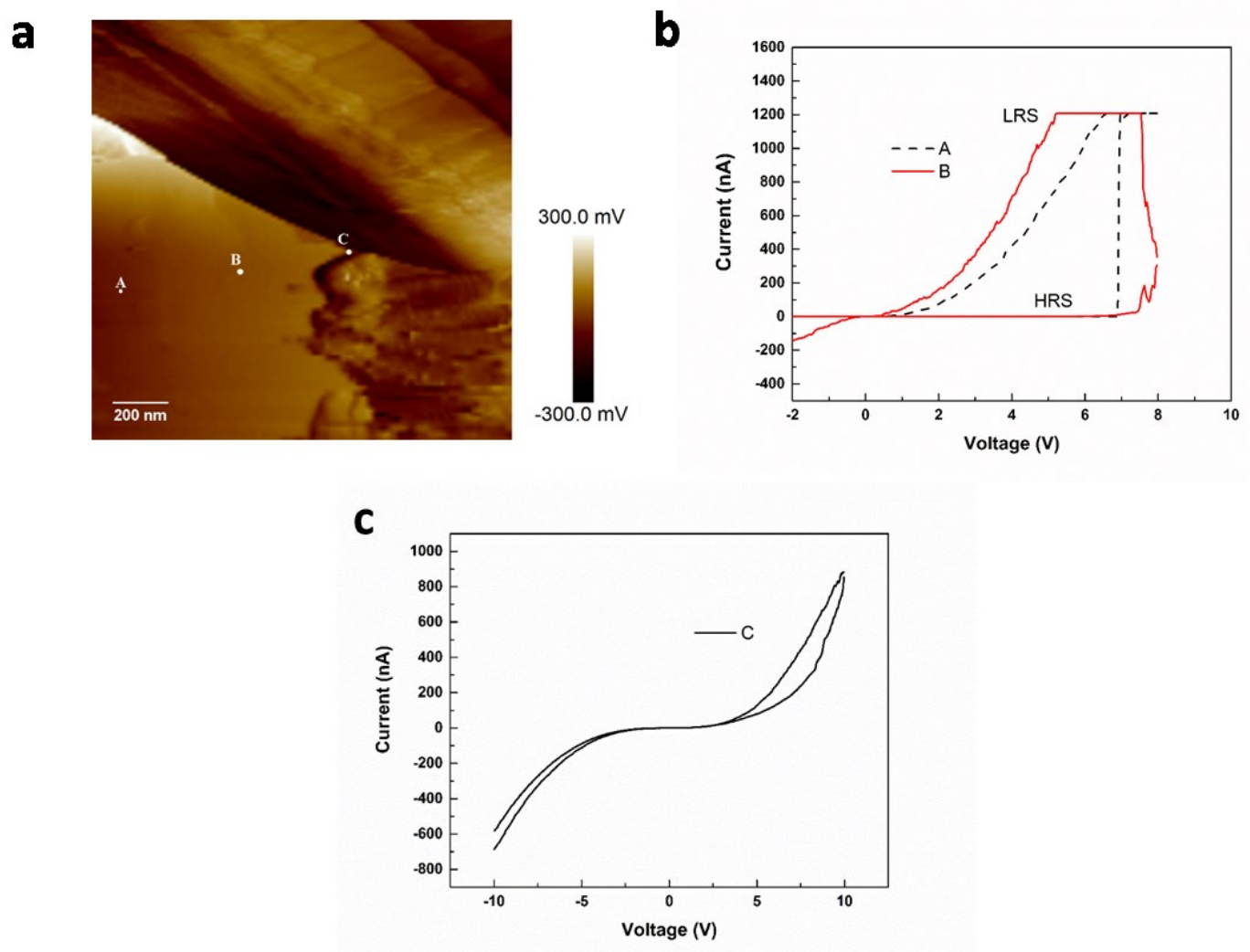

Figure S3-8. (a) Deflection image obtained on the $\mathrm{LiCoO}_{2}$ thin film. (b) Representative I/V curves obtained at spots $\mathrm{A}$ and $\mathrm{B}$ in the grain interior, and the $\mathrm{I} / \mathrm{V}$ curve (c) obtained at spot $\mathrm{C}$ along the grain boundaries. 


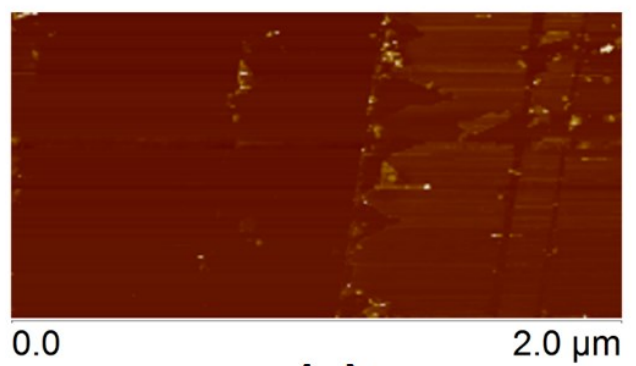

(a)

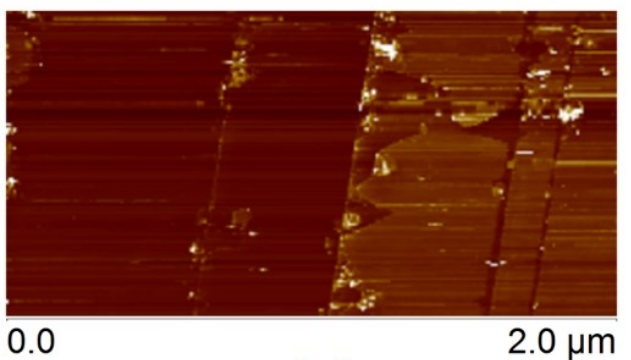

(c)

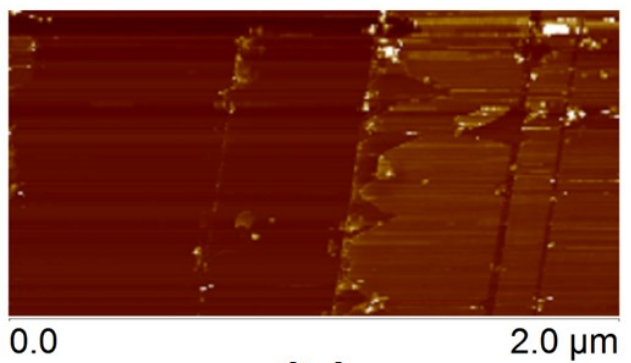

(b)

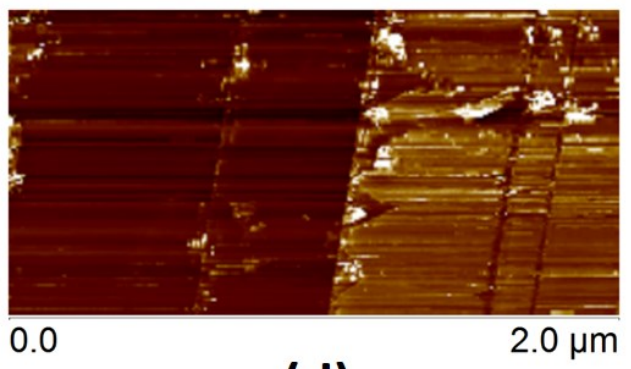

(d)
$5.0 \mathrm{nA}$

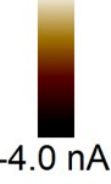

$-4.0 \mathrm{nA}$

Figure S3-9. Electrical current images obtained on the HOPG substrate under an applied force of $26.6 \mathrm{nN}$ (a), $79.9 \mathrm{nN}$ (b), $133.2 \mathrm{nN}$ (c) and $213.1 \mathrm{nN}$ (d). The applied DC bias voltage was $2 \mathrm{~V}$.

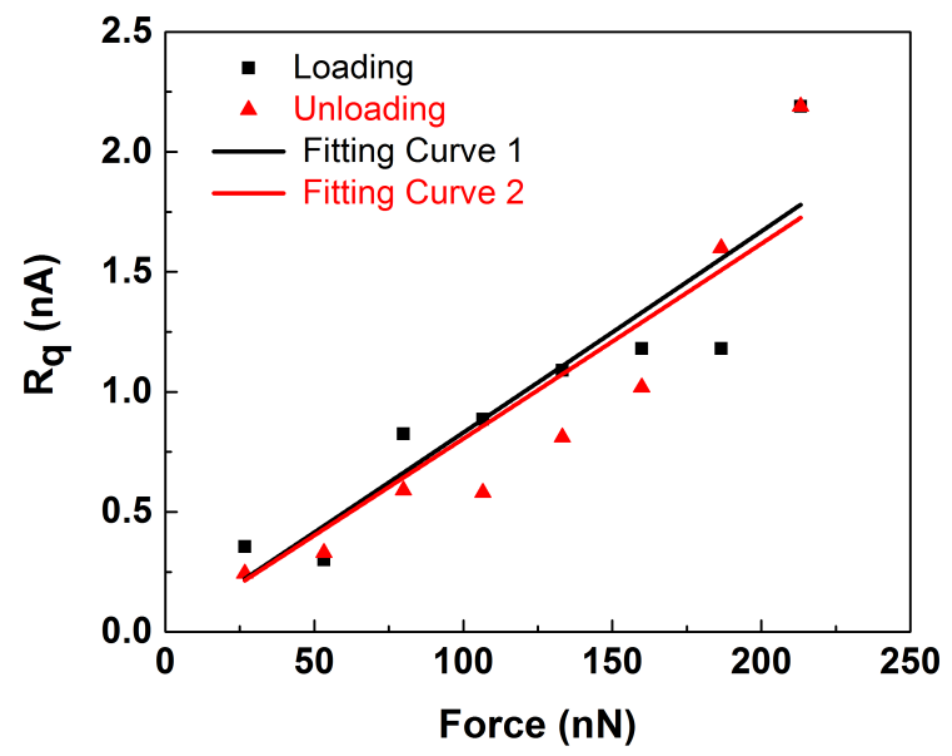

Figure S3-10. Root mean square roughness $\left(\mathrm{R}_{\mathrm{q}}\right)$ of electrical current from HOPG substrate as a function of applied forces with nonlinear curve fitting. 
a

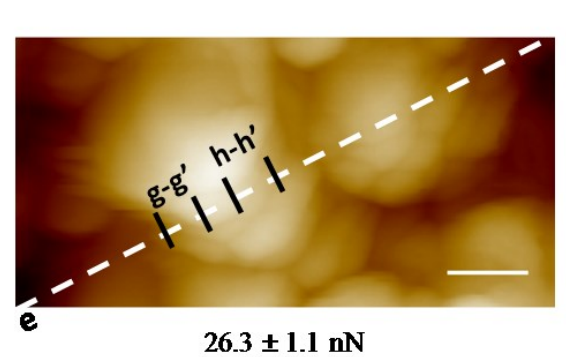

C

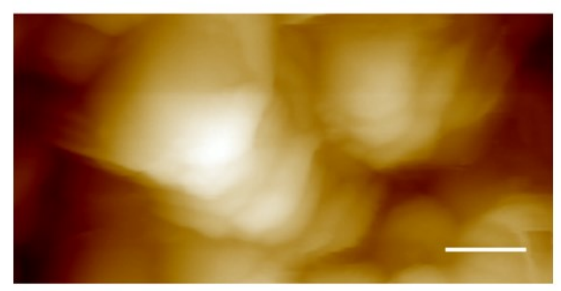

$131.6 \pm 5.4 \mathrm{nN}$ $e^{\prime b}$

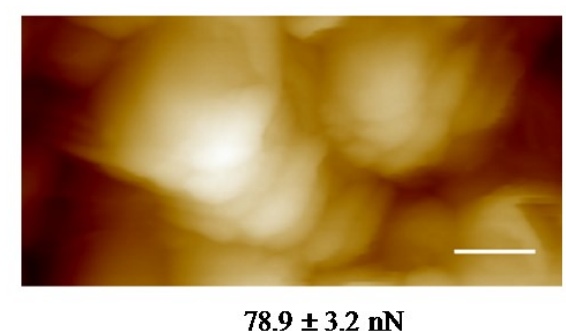

d

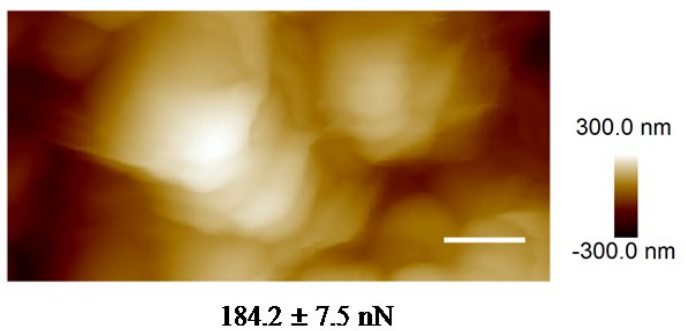

Figure S3-11. Height evolution of the corresponding area in the layered $\mathrm{LiCoO}_{2}$ substrate during the stress loading process under a bias voltage of $2 \mathrm{~V}$. Scale bars are $300 \mathrm{~nm}$.

a

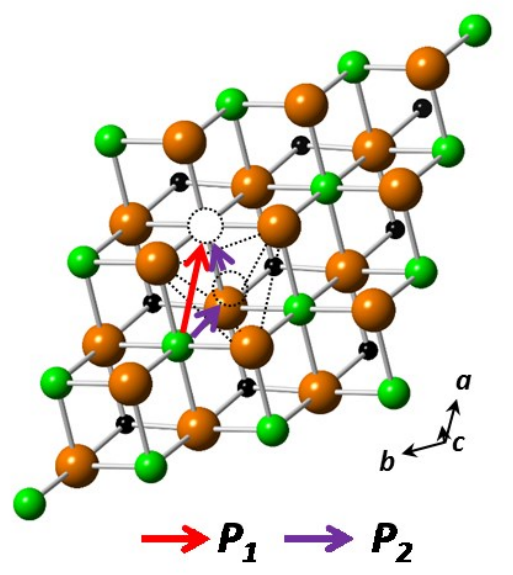

b

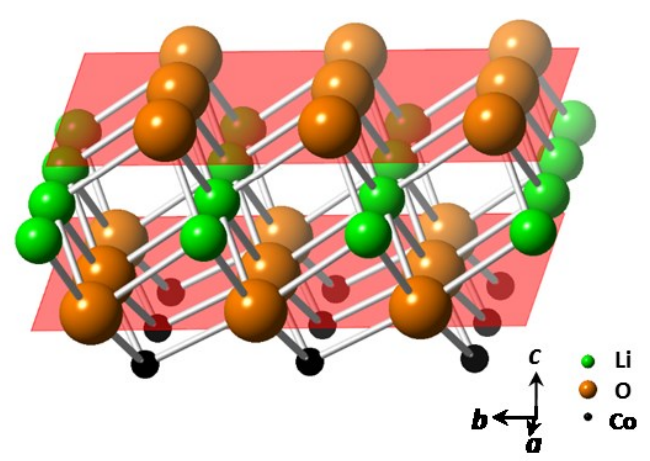

Figure S3-12. (a) Two mechanisms proposed for the diffusion of lithium ions along the (001) plane in the layered $\mathrm{LiCoO}_{2}$ substrate. (b) lateral view of the lithium ion diffusion layer between two close-packed oxygen layers. 


\section{Appendix B. Supporting information for Chapter 4}

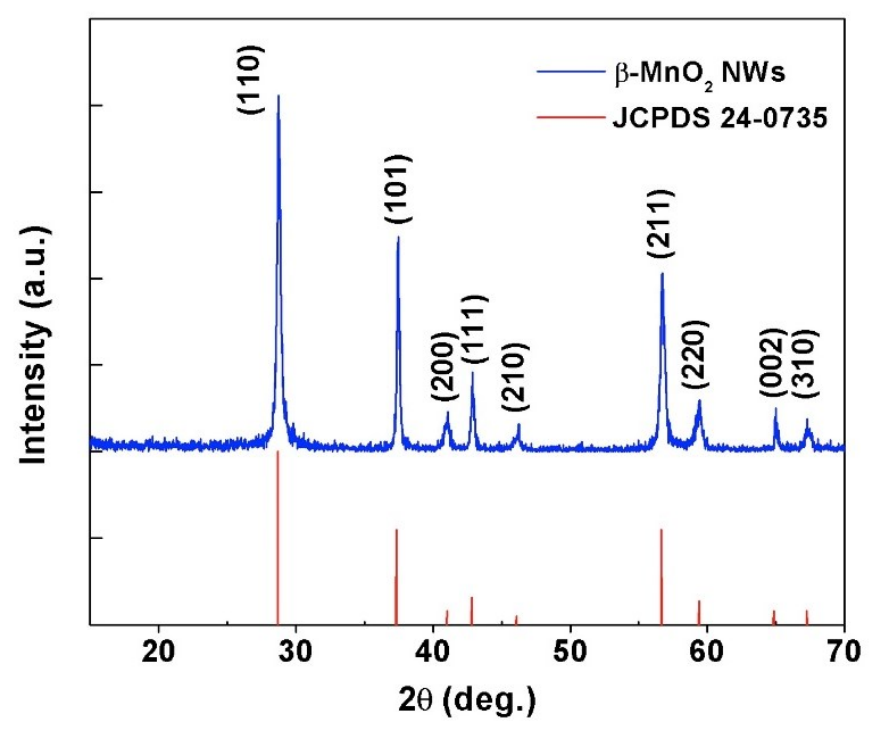

Figure S4-1 XRD pattern of the $\beta-\mathrm{MnO}_{2}$ nanowires obtained at $12 \mathrm{~h}$ with good agreement with standard JCPDS 24-0735.

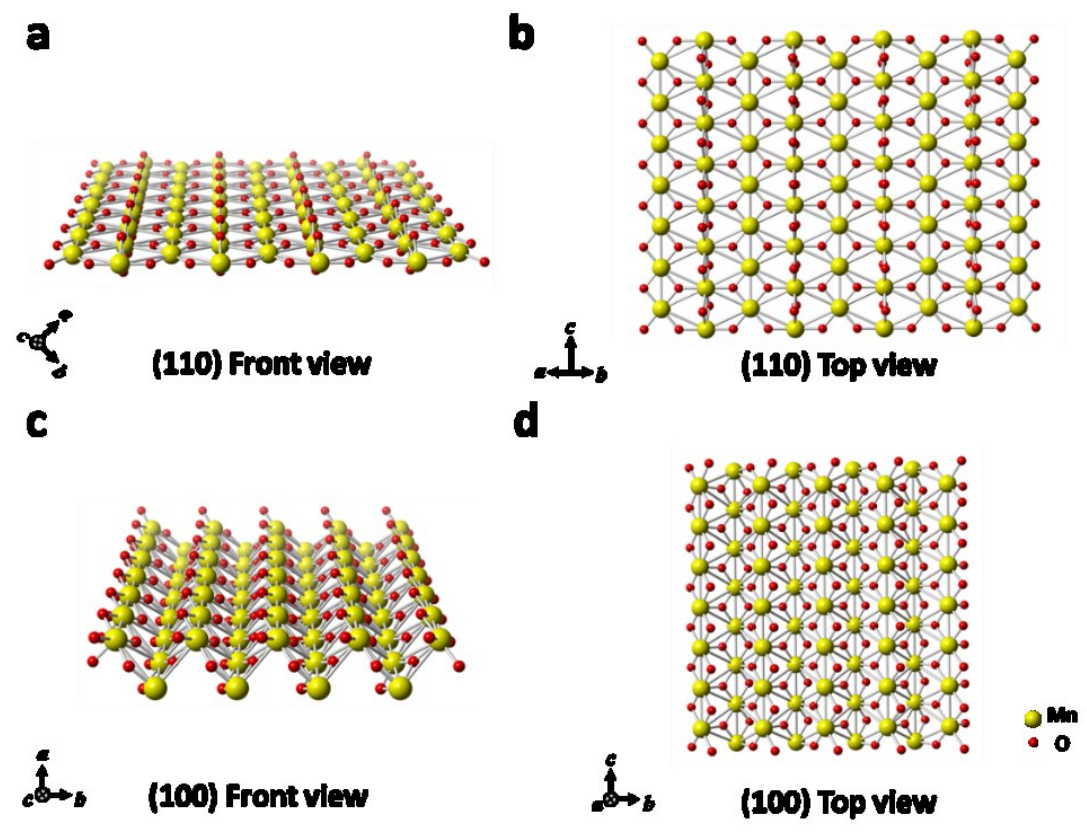

Figure S4-2 The front view (a) and top view (b) of the atoms arrangement on the (110) surfaces. The front view (c) and top view (d) of the atoms arrangement on the (100) surfaces. 


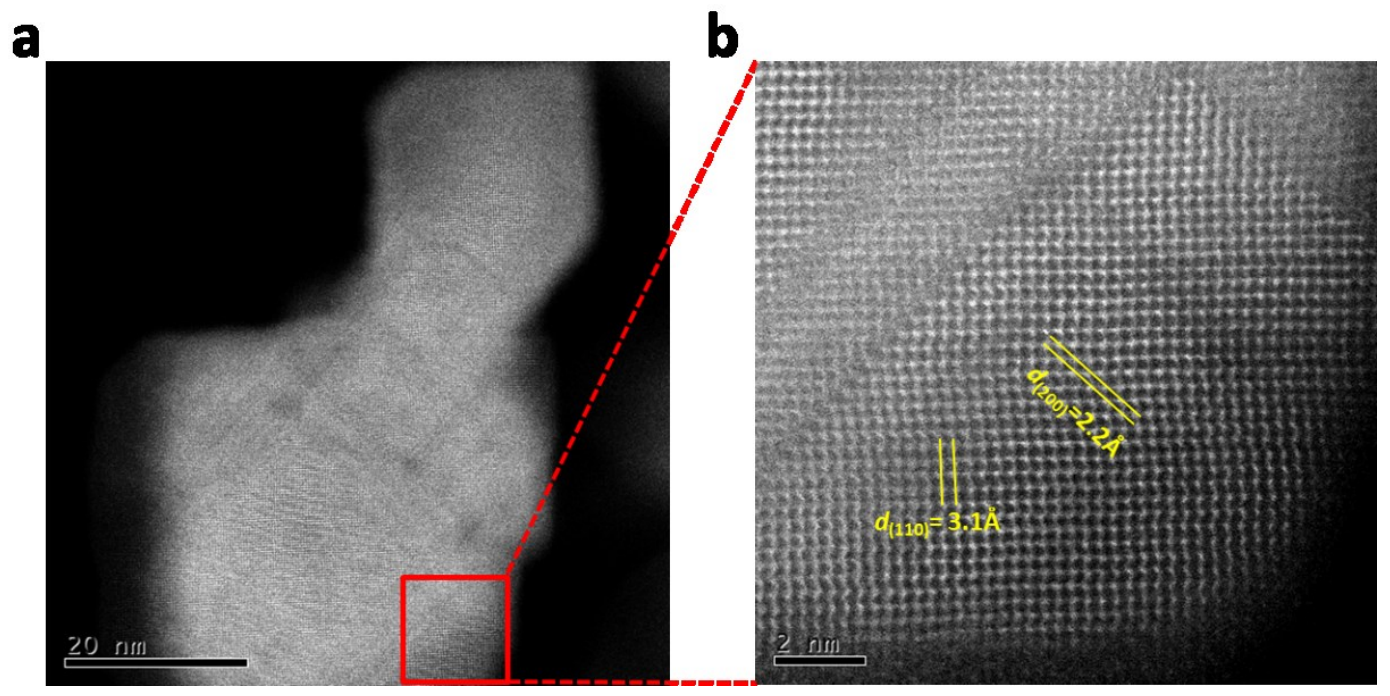

Figure S4-3 (a) HAADF cross-section image of two attached $\beta-\mathrm{MnO}_{2}$ nanowires viewed along the [001] direction. (b) Atomic resolution HAADF image of the line defects along the (100) lateral surfaces.

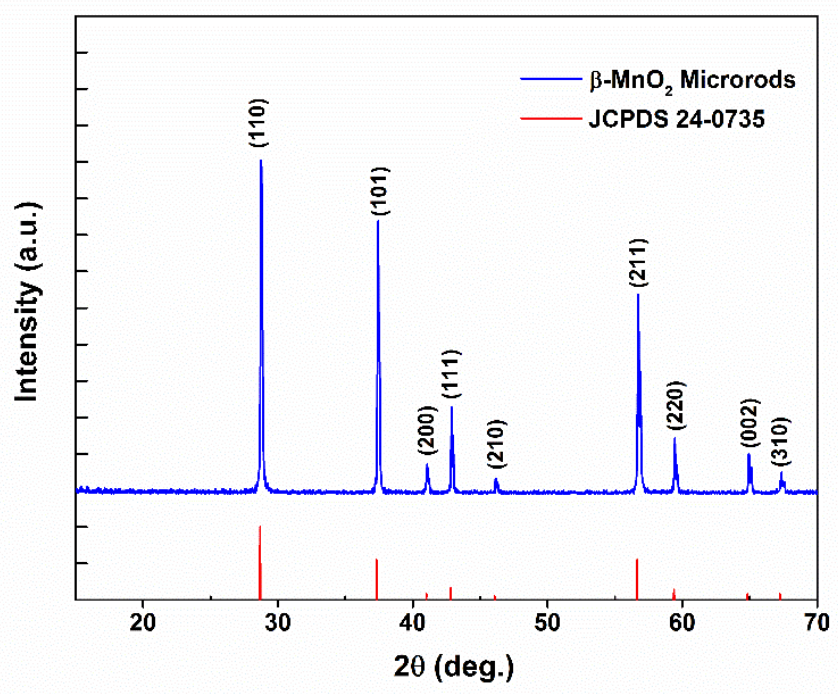

Figure S4-4 The powder XRD pattern of the $\beta-\mathrm{MnO}_{2}$ microrods (12 h) compared with the standard JCPDS 24-0735. 

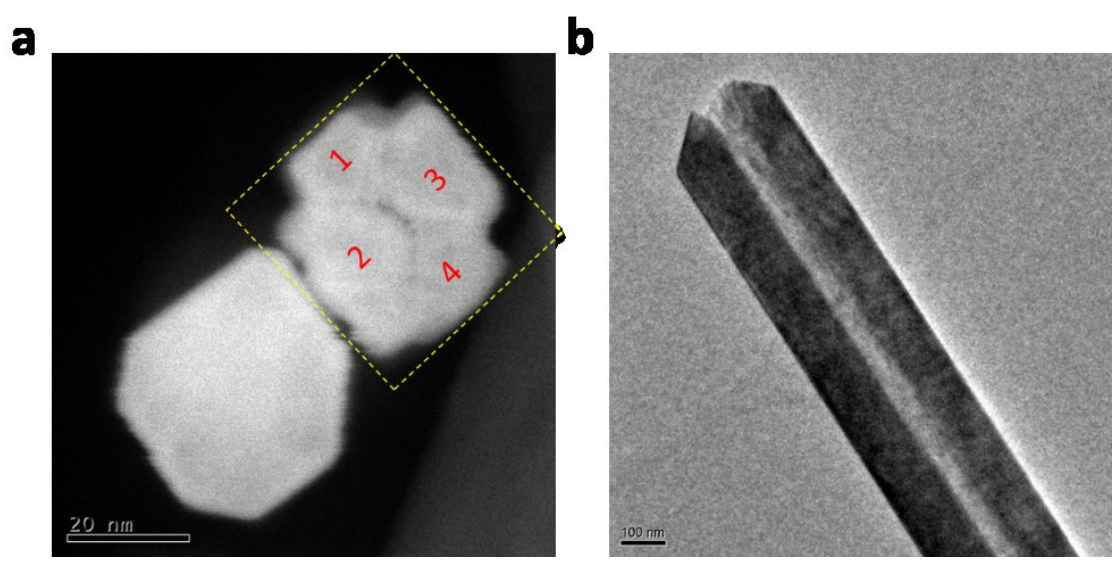

Figure S4-5 (a) HAADF cross-section image of fourfold attached $\beta-\mathrm{MnO}_{2}$ nanowires viewed along the [001] direction. (b) Low magnification TEM image of a $\beta-\mathrm{MnO}_{2}$ nanowire with fourfold oriented attachment viewed from the lateral side. 


\section{Appendix C. Supporting information for Chapter 5}

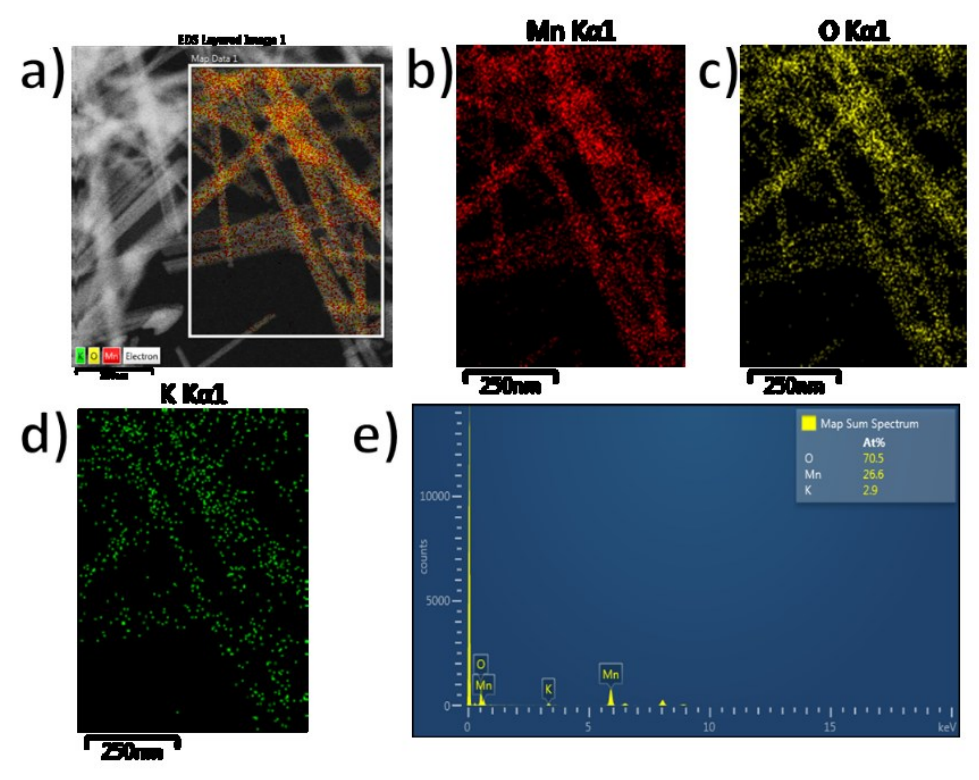

Figure S5-1 (a) HAADF image of $\alpha-\mathrm{MnO}_{2}$ nanowires and the corresponding EDS mapping of (b) $\mathrm{Mn}$; (c) $\mathrm{O}$; and (d) $\mathrm{K}$ element. (e) Elemental percentage of the $\alpha-\mathrm{K}_{0.5} \mathrm{Mn}_{4} \mathrm{O}_{8}$ nanowires.

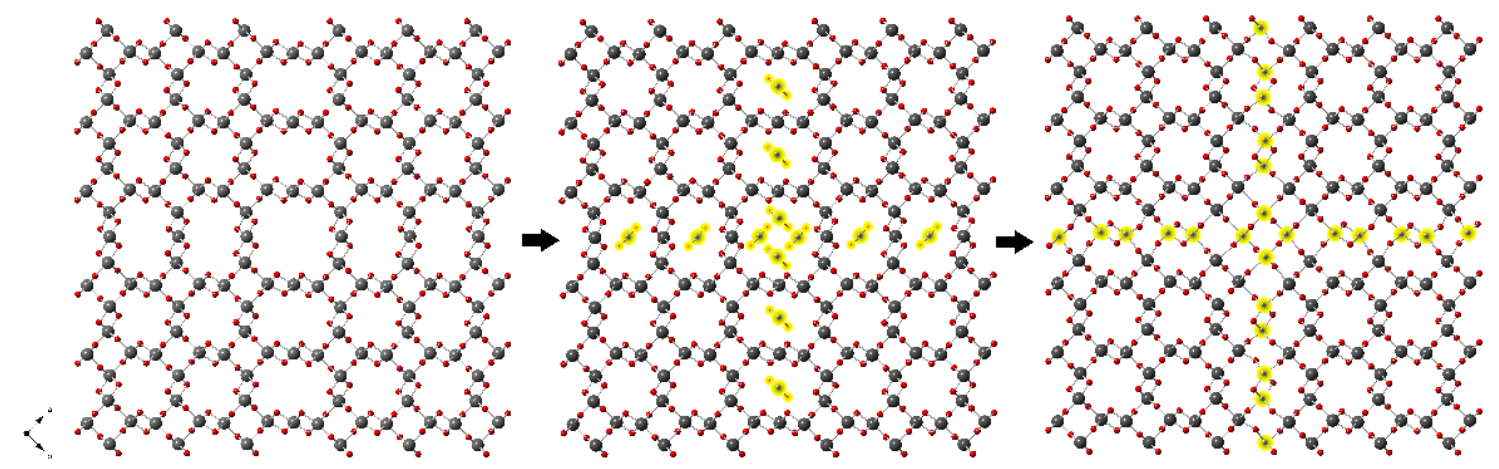

Figure S5-2 Atom models illustrating the proposed tunnel transition from $[2 \times 3]$ tunnels to $[1 \times 2]$ tunnels. 


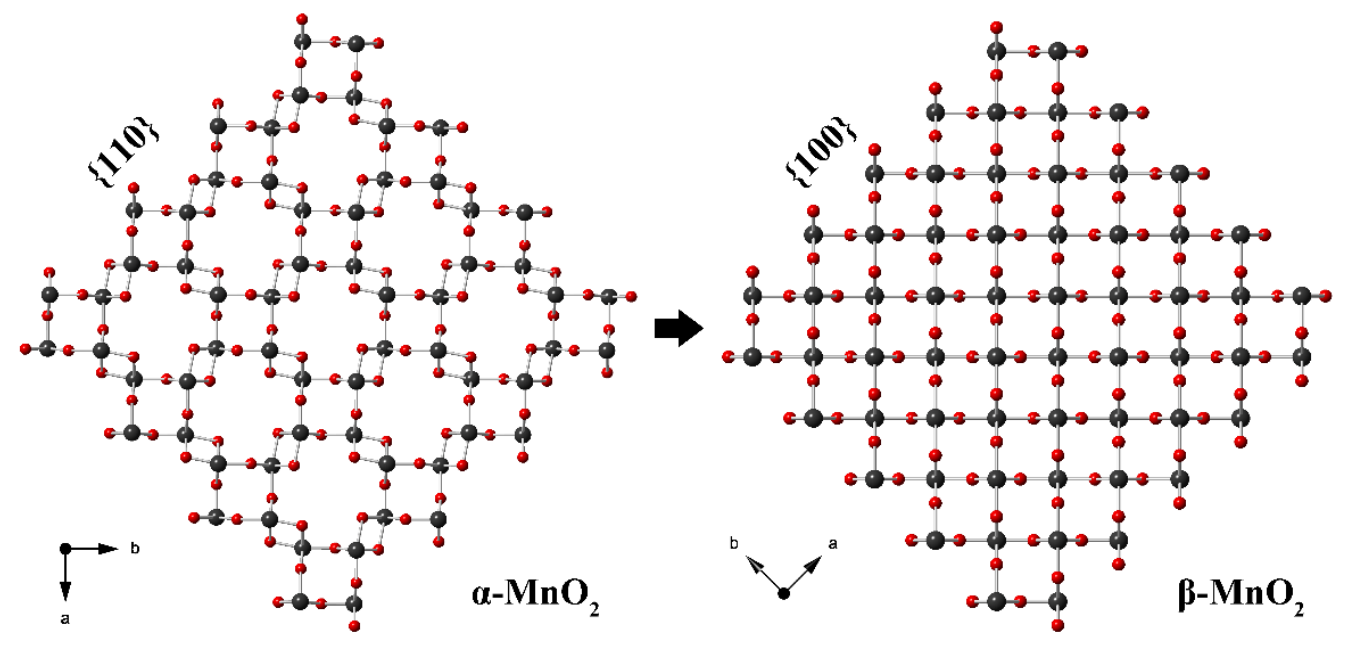

Figure S5-3 Atom models illustrating the similar arrangement of $\mathrm{Mn}$ and $\mathrm{O}$ atoms on the $\{110\}$ plane of $\alpha-\mathrm{MnO}_{2}$ and $\{100\}$ plane of $\beta-\mathrm{MnO}_{2}$.

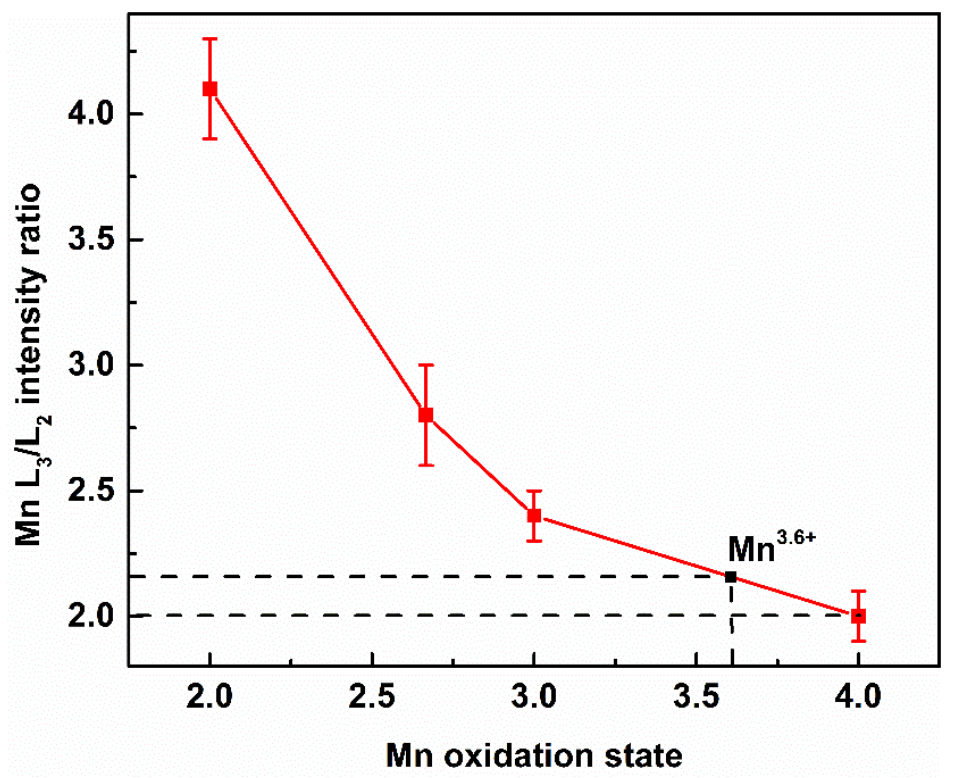

Figure S5-4 Averaged Mn valence state identification based on the white-line intensity ratio $\left(\mathrm{L}_{3} / \mathrm{L}_{2}\right)$. 

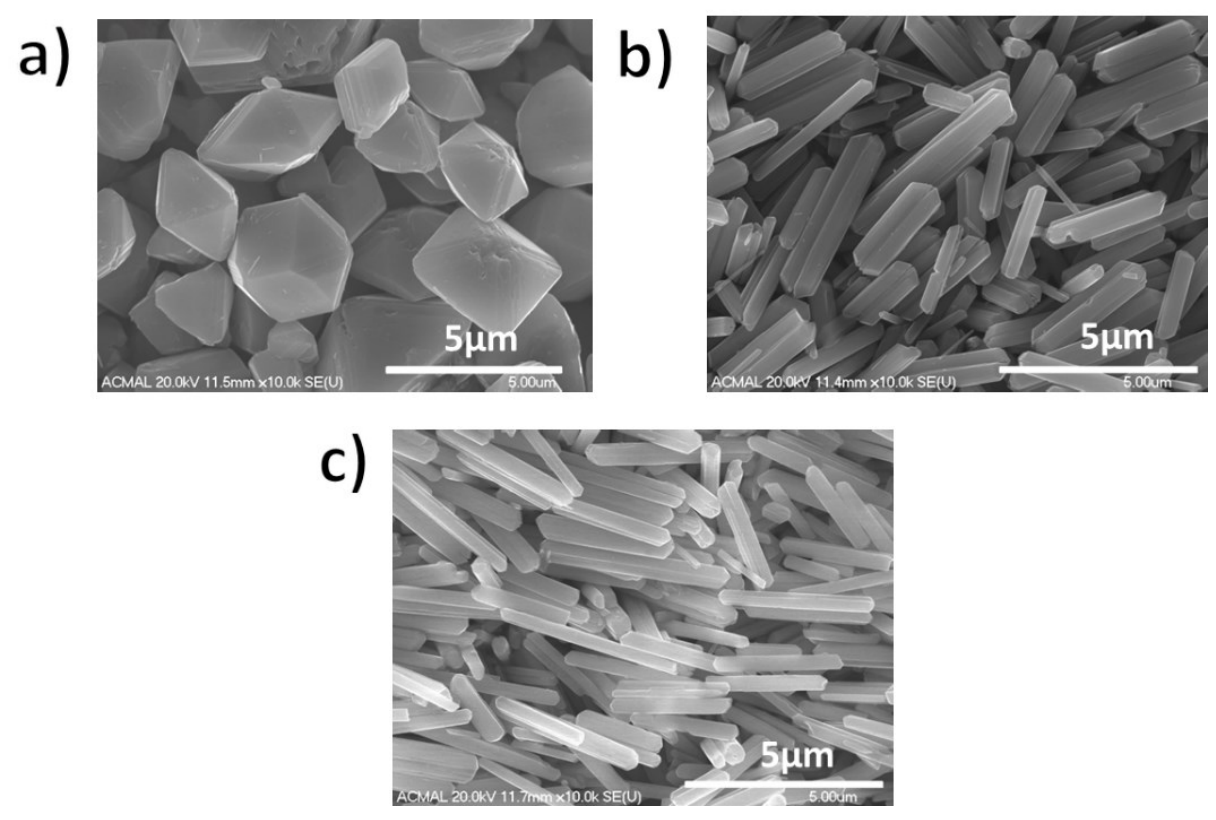

Figure S5-5 FE-SEM images of the $\beta-\mathrm{MnO}_{2}$ structures obtained with different surfactants: (a) $0.09 \mathrm{M} \mathrm{KNO}_{3}$; (b) $0.09 \mathrm{M} \mathrm{NaCl}$; (c) $0.09 \mathrm{M} \mathrm{NH}_{4} \mathrm{Cl}$.

a)

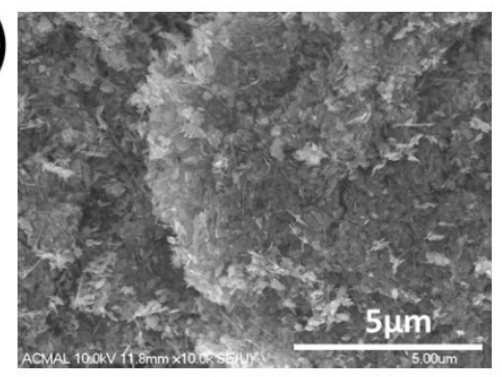

c)

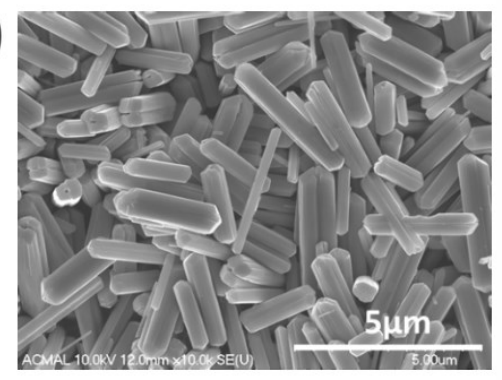

b)

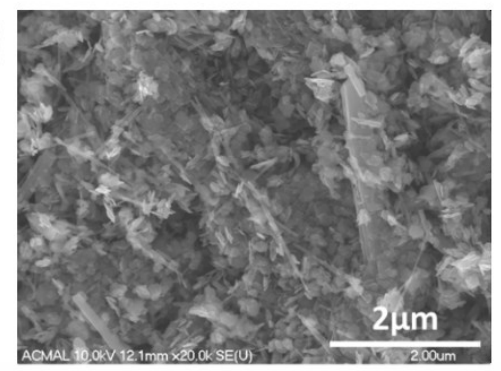

d)

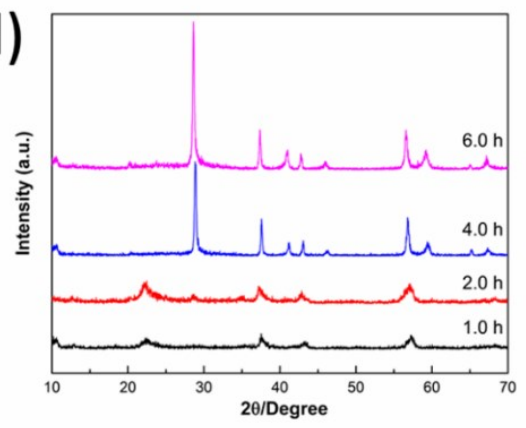

Figure S5-6 FE-SEM images of the $\mathrm{MnO}_{2}$ structures obtained with $0.02 \mathrm{M} \mathrm{KCl}$ surfactant under different hydrothermal reaction time: (a) 1h; (b) 2h; (c) 4h. (d) The corresponding XRD patterns indicating the formation of $\gamma-\mathrm{MnO}_{2}$ intermediate phase. The phase of $\mathrm{MnO}_{2}$ structures obtained at $1 \mathrm{~h}$ and $2 \mathrm{~h}$ was identified to $\alpha-\mathrm{MnO}_{2}$ (JCPDS 44-0141) and $\gamma-$ $\mathrm{MnO}_{2}$ (JCPDS 14-0644). 
a)

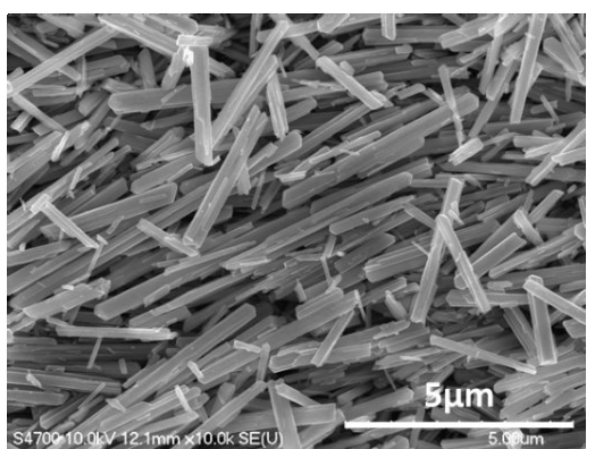

b)

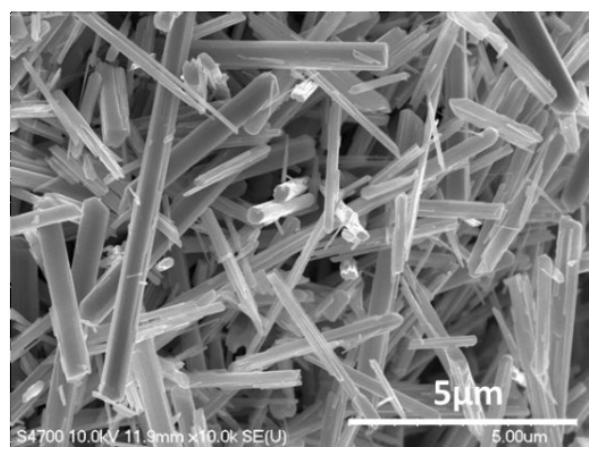

Figure S5-7 FE-SEM images of $\beta-\mathrm{MnO}_{2}$ structures obtained by redox reactions between $\mathrm{KMnO}_{4}$ and $\mathrm{MnCl}_{2} \cdot 4 \mathrm{H}_{2} \mathrm{O}$ with a molar ratio of (a) 1:3 and (b) 1:6. To ensure the concentrations of $\mathrm{K}^{+}$cations in the solution were identical to that of $0.09 \mathrm{M} \mathrm{KCl}(2: 3$ molar ratio), $0.11 \mathrm{M}$ and $0.12 \mathrm{M} \mathrm{KCl}$ were added respectively to the reactor. The reaction conditions were controlled the same at $180^{\circ} \mathrm{C}$ for $12 \mathrm{hrs}$.

\section{a)}
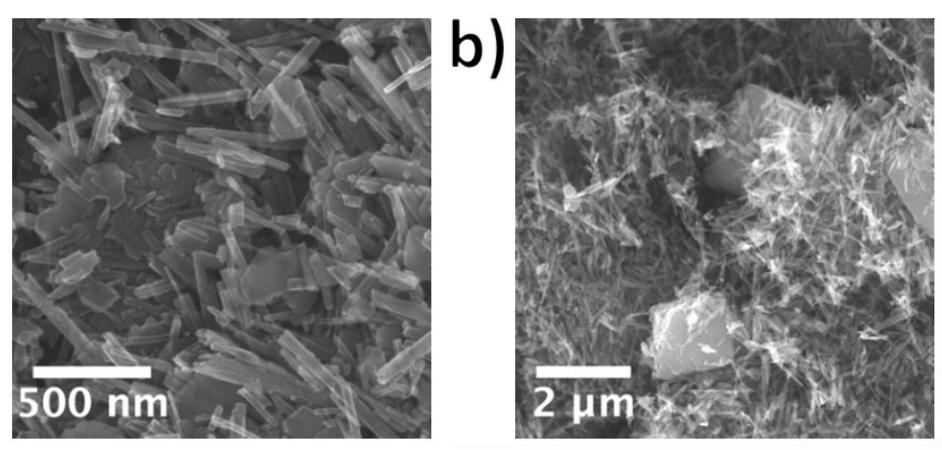

c)
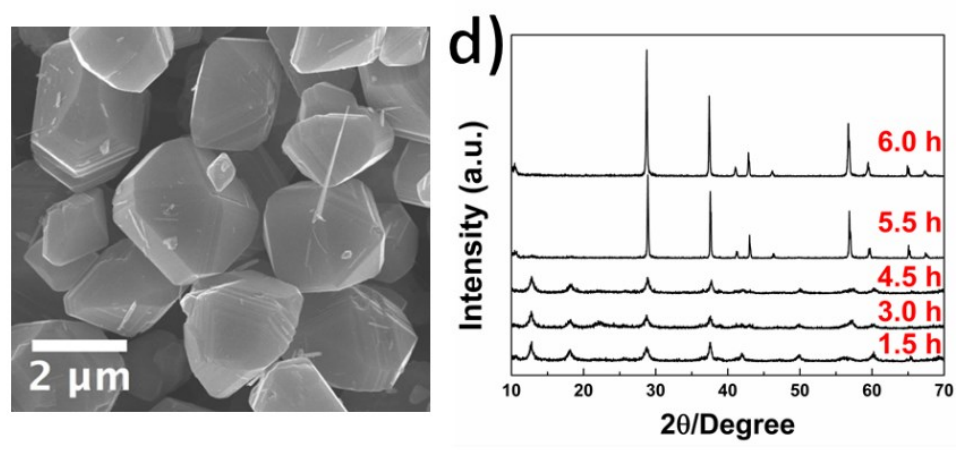

Figure S5-8 FE-SEM images of the $\mathrm{MnO}_{2}$ structures obtained with $0.09 \mathrm{M} \mathrm{KCl}$ surfactant under different hydrothermal reaction time: (a) $3.0 \mathrm{~h}$; (b) $4.5 \mathrm{~h}$; (c) $5.5 \mathrm{~h}$. (d) The corresponding XRD patterns indicating the formation of $\alpha-\mathrm{MnO}_{2}$ intermediate phase (JCPDS 44-0141) before phase transition to $\beta-\mathrm{MnO}_{2}$. 


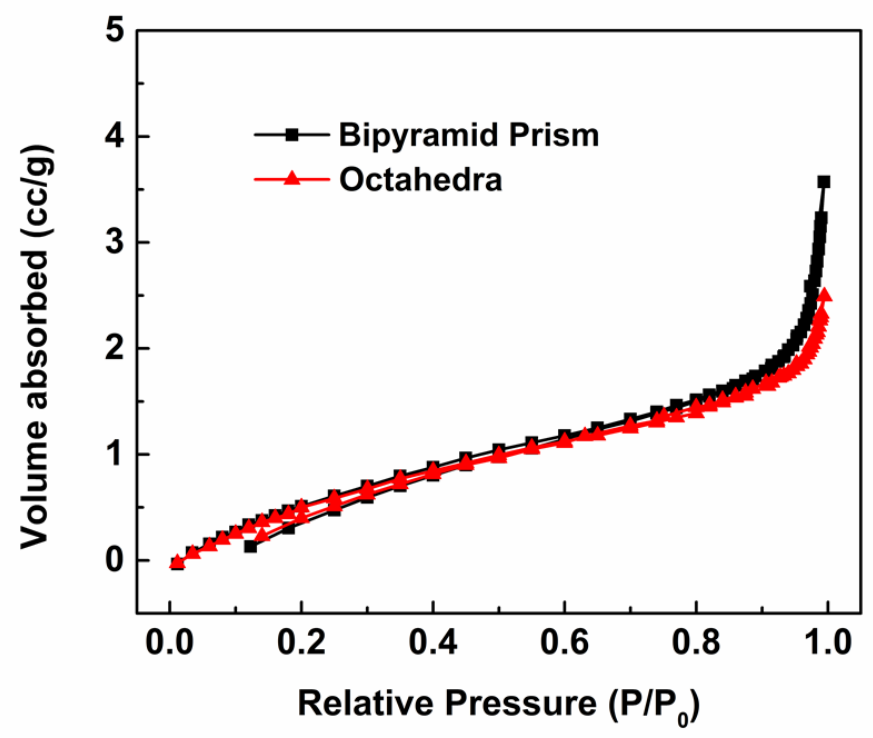

Figure S5-9 The $\mathrm{N}_{2}$ adsorption-desorption isothermals for the $\beta-\mathrm{MnO}_{2}$ obtained with 0.04 $\mathrm{M}$ (bipyramid prism) and $0.08 \mathrm{M} \mathrm{KCl}$ (octahedra) surfactant.
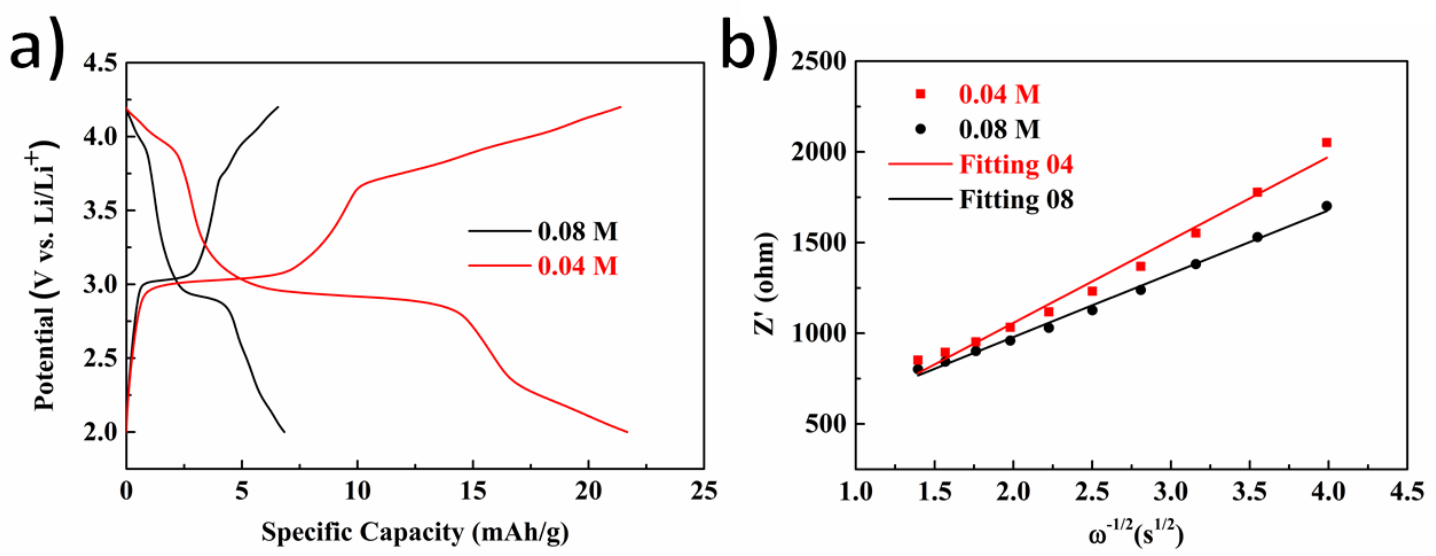

Figure S5-10 (a) Representative charge-discharge curves of $\beta-\mathrm{MnO}_{2}$ with different morphologies; (b) Calculation of Warburg coefficient by linear fitting of the real impedance ( $\left.Z^{\prime}\right)$ versus $\omega^{-1 / 2}$. 
Table S5-1 The fitted impedance parameters for the Nyquist plots obtained before cycling.

\begin{tabular}{|c|c|c|c|c|c|}
\hline Samples & $\begin{array}{l}\text { R1 } \\
(\Omega)\end{array}$ & $\begin{array}{l}\text { R2 } \\
(\Omega)\end{array}$ & $\begin{array}{l}\text { R3 } \\
(\Omega)\end{array}$ & $\begin{array}{c}\text { W1 } \\
\left(\Omega \mathrm{s}^{-1 / 2}\right)\end{array}$ & $\begin{array}{c}\text { Warburg } \\
\text { coefficient } \\
(\sigma) \\
\Omega \cdot \mathbf{s}^{-1 / 2}\end{array}$ \\
\hline 0.04 M & 2.64 & 46.63 & 453.6 & 239.8 & 457.81 \\
\hline 0.08 M & 3.15 & 18.6 & 477.7 & 177.9 & 349.48 \\
\hline
\end{tabular}

Table S5-2 Simulation parameters for each system

\begin{tabular}{ccc}
\hline System & Space group & $\begin{array}{c}\text { K-point grid } \\
\text { spacing }\end{array}$ \\
\hline $\mathrm{K}$ & $\mathrm{Im}-3 \mathrm{~m}(229)$ & $8 \times 8 \times 8$ \\
$\mathrm{Mn}$ & $\mathrm{I}-43 \mathrm{~m}(217)$ & $14 \times 12 \times 12$ \\
$\mathrm{O}$ & $\mathrm{B} 2 / \mathrm{m}(12)$ & $5 \times 5 \times 5$ \\
$\alpha-\mathrm{MnO}_{2}$ & $\mathrm{I} 4 / \mathrm{m}(87)$ & $5 \times 5 \times 17$ \\
$\beta-\mathrm{MnO}_{2}$ & $\mathrm{P} 42 / \mathrm{mnm}(136)$ & $14 \times 14 \times 21$ \\
$\mathrm{Ortho}_{2}-\mathrm{MnO}_{2}$ & $\mathrm{Pbnm}(62)$ & $7 \times 3 \times 10$ \\
$\mathrm{~K}_{x} \mathrm{Mn}_{8} \mathrm{O}_{16}$ & $\mathrm{I} 4 / \mathrm{m}(87)$ & $5 \times 5 \times 17$ \\
\hline
\end{tabular}




\section{Appendix D. Supporting information for Chapter 7}

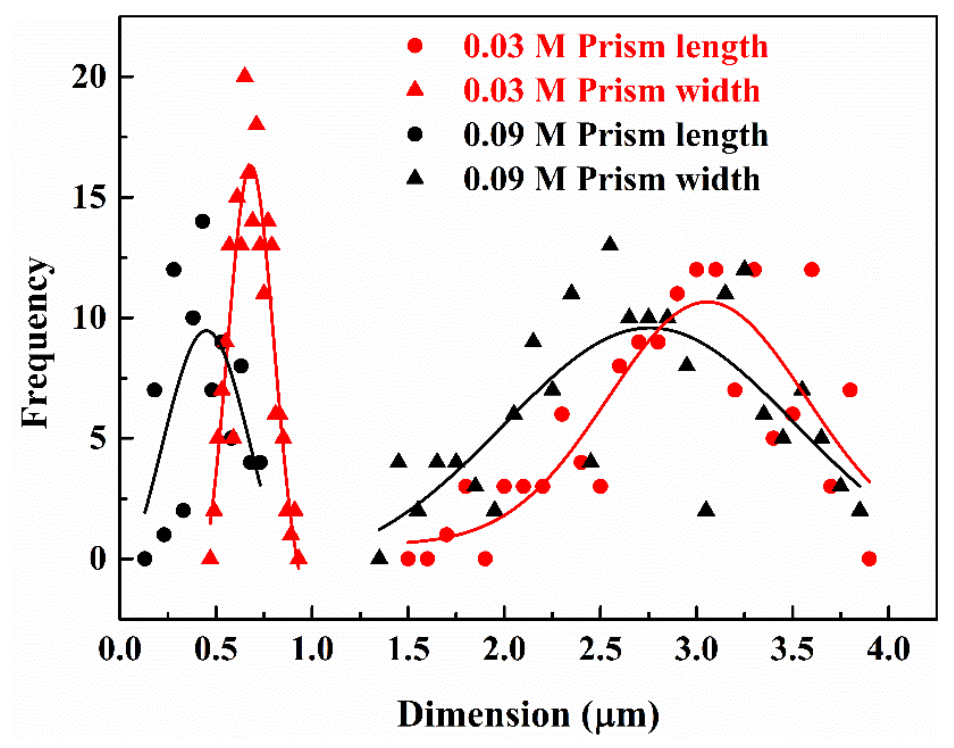

Figure S6-1 Statistical analysis for the distribution of prism length and prism width for the bipyramid prism $(0.03 \mathrm{M})$ and octahedron $(0.09 \mathrm{M})$ beta $\mathrm{MnO}_{2}$ structures.

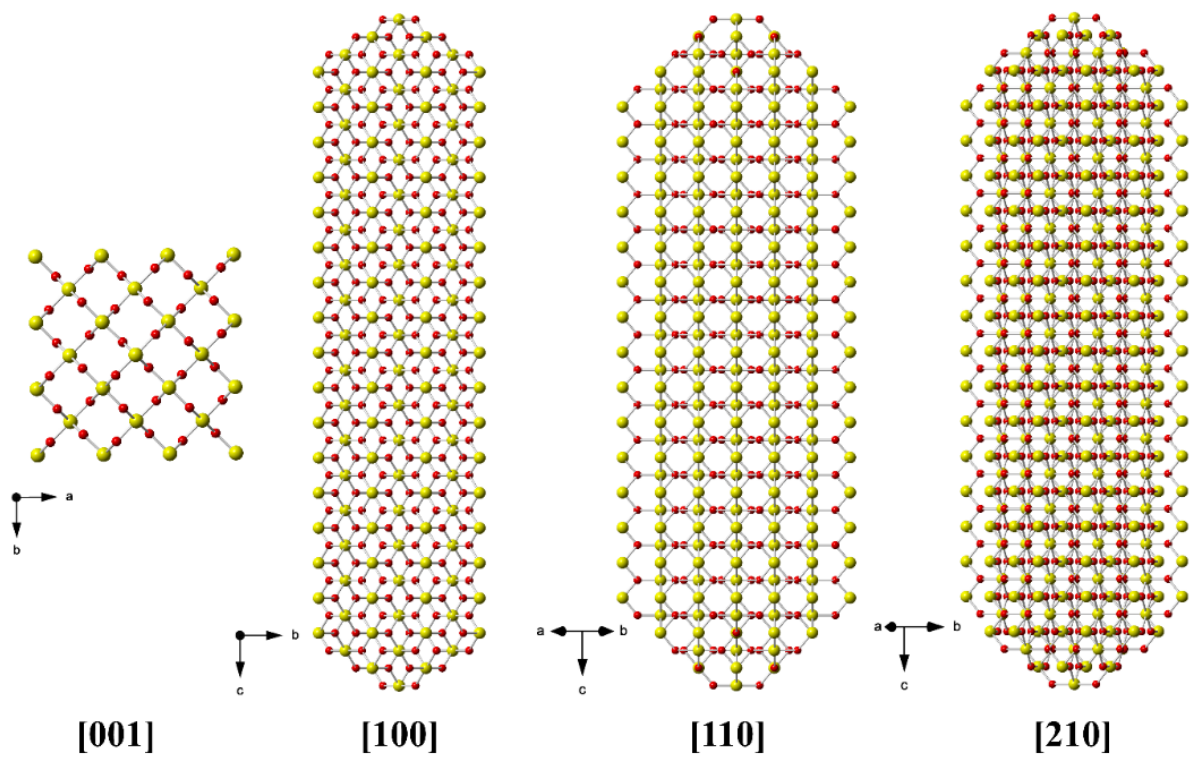

Figure S6-2 Atom model illustrates the same beta $\mathrm{MnO}_{2}$ nanowire viewed along [001] tunnel direction and [100], [110], [210] lateral zone axis. 

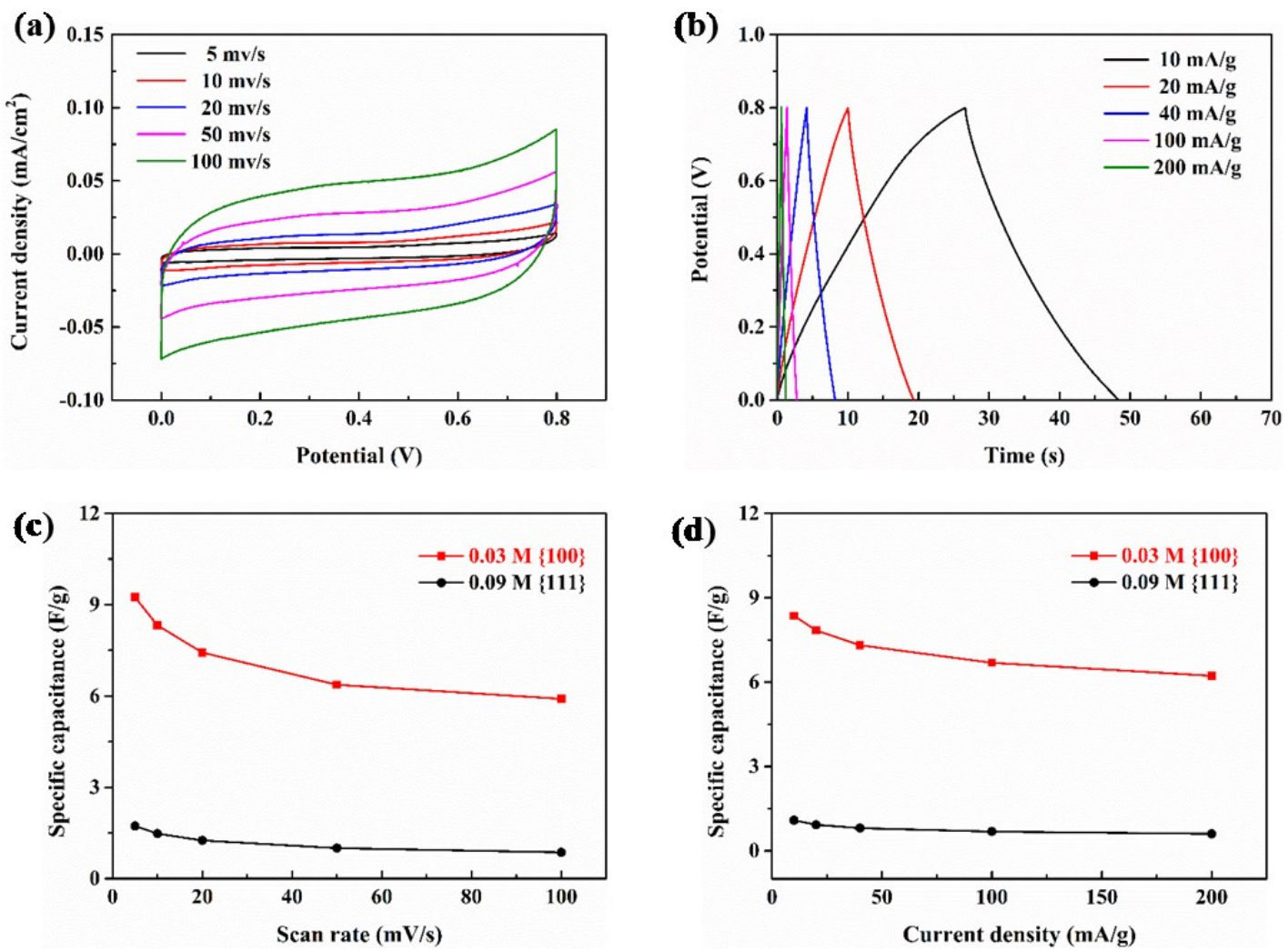

Figure S6-3 Electrochemical performance of beta $\mathrm{MnO}_{2}$ symmetric supercapacitor with $1.0 \mathrm{~mol} / \mathrm{L} \mathrm{Na}_{2} \mathrm{SO}_{4}$ aqueous electrolyte. (a) $\mathrm{CV}$ curves of the beta- $\mathrm{MnO}_{2}$ octahedra $(0.09 \mathrm{M}-$ $\{111\})$ recorded under different scan rates, (b) galvanostatic charge-discharge of the beta$\mathrm{MnO}_{2}$ octahedra $(0.09 \mathrm{M}-\{111\})$ at different current densities, and variation of specific capacitances of beta $\mathrm{MnO}_{2}$ bipyramid prism $(0.03 \mathrm{M}-\{100\})$ and octahedra (0.09M-\{111\}) against different (c) scan rates and (d) current densities. 


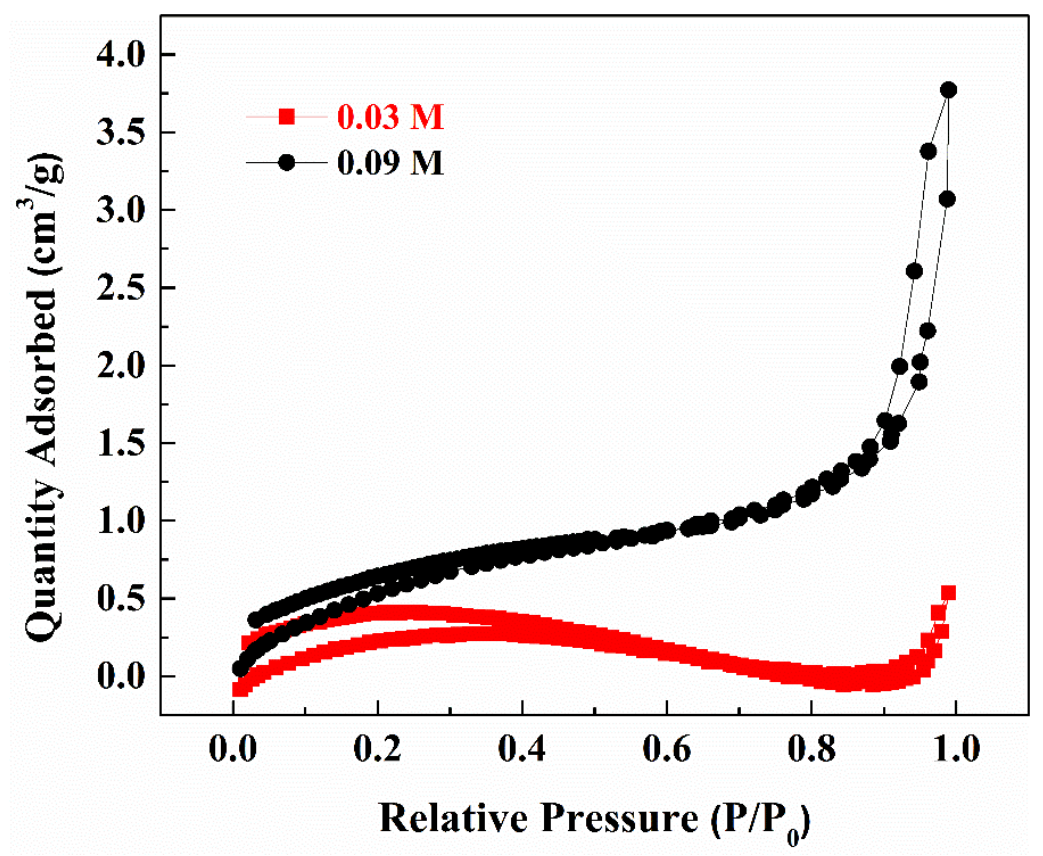

Figure S6-4 The $\mathrm{N}_{2}$ adsorption-desorption isothermals for the $\beta-\mathrm{MnO}_{2}$ obtained with $0.03 \mathrm{M}$ (bipyramid prism) and $0.09 \mathrm{M} \mathrm{KCl}$ (octahedra) surfactant. 